\title{
Theoretische Beschreibung und experimentelle Untersuchung raum-zeitlicher Strukturbildung in akustischen Kavitationsblasenfeldern
}

\author{
Dissertation \\ zur Erlangung des Doktorgrades \\ der Mathematisch-Naturwissenschaftlichen Fakultäten \\ der Georg-August-Universität zu Göttingen
}

vorgelegt von

Stefan Luther

aus

Springe

Göttingen 2000 
D7

Referent:

Prof. Dr. Werner Lauterborn

Korreferent:

Prof. Dr. Dirk Ronneberger

Tag der mündlichen Prüfung:

20. Juni 2000 


\section{Zusammenfassung}

Das Thema dieser Arbeit ist die raum-zeitliche Strukturbildung in akustischen Kavitationsblasenfeldern. Die zentrale Fragestellung ist die Bedeutung der mikroskopischen Dynamik der Blasen auf schnellen Zeitskalen für das Entstehen und die Stabilität von makroskopischen Strukturen einer Vielzahl von individuellen Blasen auf langsamen Zeitskalen. Dazu werden experimentell die Trajektorien einzelner Blasen im Schallfeld bestimmt und eine theoretische Beschreibung der Bewegung der Blasen und ihrer Wechselwirkungen gegeben.

Im ersten Teil der Arbeit werden die experimentellen Methoden eingeführt, mit Hilfe derer die Positionen, Geschwindigkeiten und Radien der Blasen gemessen werden. Mit Hilfe von Bildbearbeitungsalgorithmen können die Trajektorien der Blasen über eine Bildsequenz verfolgt werden.

Die experimentellen Daten werden im zweiten Teil der Arbeit ausgewertet und diskutiert.

Auf der Grundlage der experimentellen Untersuchungen wird das System im dritten Teil der Arbeit theoretisch beschreiben. Eine makroskopische Theorie der Schallausbreitung in Flüssigkeits-Blasen-Gemischen wird numerisch untersucht. Es wird eine Selbstfokussierung der Schallwelle und eine Selbstkonzentration der Blasen beobachtet.

Eine theoretische Beschreibung der nichtlinearen Blasenschwingungen und der mikroskopischen Blasenwechselwirkung wird basierend auf einer Lagrangschen Formulierung der inkompressiblen Hydrodynamik entwickelt. Es wird gezeigt, daß die Kopplung von Translation und Oszillation einer Einzelblase zu einer komplexen Schwerpunktsbewegung führt. Ein Kriterium für die sphärische Stabilität wird diskutiert.

Die Kräfte zwischen den Blasen ändern sich signifikant, wenn die Kompressibilität der Flüssigkeit berücksichtigt wird. Für ein Modellsystem delay-gekoppelter Blasenoszillatoren wird dieser allgemeine Effekt demonstriert.

Abschließend wird die akustische Emission eines getrieben Kavitationsblasenfeldes mit den Methoden der nichtlinearen Zeitreihenanalyse untersucht und die Dimension dieses dynamischen Systems abgeschätzt. 


\section{Inhaltsverzeichnis}

1. Einleitung 1

$\begin{array}{ll}\text { 2. Experiment: Meßmethoden } & 7\end{array}$

2.1. Übersicht über Meßverfahren in der Strömungsmechanik . . . . . . . . . . . . 7

2.2. Experimenteller Aufbau . . . . . . . . . . . . . . . . . . . . . . . . . 10

2.3. Das Bildaufnahmesystem . . . . . . . . . . . . . . . . . . 13

2.3.1. Hardware-Komponenten . . . . . . . . . . . . . . . . . 14

2.3.2. Funktion . . . . . . . . . . . . . . . . . 16

2.3.3. Modellierung . . . . . . . . . . . . . . . . . . . . 17

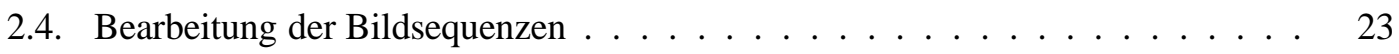

2.4.1. Bildvorbearbeitung . . . . . . . . . . . . . 23

2.4.2. Bildbearbeitung . . . . . . . . . . . . . . . . . . . . 24

2.5. Analyse der Bildsequenzen . . . . . . . . . . . . . . . . . . . 26

2.5.1. Klassifikation ..................... 26

2.5.2. Bewegungsanalyse . . . . . . . . . . . . . . . . . . . . . . . 29

2.6. Radienmessung . . . . . . . . . . . . . . . . . . . . . . . . . . . . 34

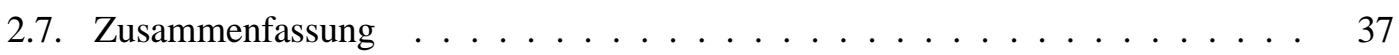

3. Experiment: Meßergebnisse und Auswertung 39

3.1. Phänomenologische Beschreibung . . . . . . . . . . . . . . 39

3.2. Methodik der Messungen . . . . . . . . . . . . . . . . . . . 41

3.3. Auswertung der Messungen . . . . . . . . . . . . . . . . . . 42 
3.3.1. Verteilung der Orte . . . . . . . . . . . . . . . . . . . . 42

3.3.2. Verteilung der Geschwindigkeiten . . . . . . . . . . . . . . . 43

3.3.3. Verteilung der Radien . . . . . . . . . . . . . . . . . . . 43

3.3.4. Trajektorien . . . . . . . . . . . . . . . . . 47

3.3.5. Blasen-Cluster . . . . . . . . . . . . . . . . 48

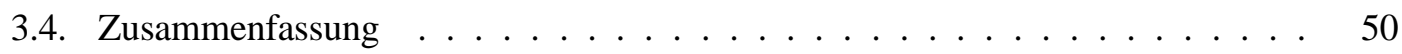

4. Theorie 52

4.1. Grundlagen der Modellierung . . . . . . . . . . . . . . . . . . . . . 52

4.1.1. Definition der Kennzahlen _ . . . . . . . . . . . . . . . . 53

4.1.2. Modellierungsansätze . . . . . . . . . . . . . . . . . 54

4.2. Kontinuumsmodell . . . . . . . . . . . . . . . . . . 55

4.2.1. Wellengleichung für Blasen-Flüssigkeits-Gemische . . . . . . . . . 55

4.2.2. Schallfeldamplitude .................... 55

4.2.3. Blasengeschwindigkeit . . . . . . . . . . . . . 56

4.2.4. Blasendichte ..................... 57

4.2.5. Lineare Stabilitätsanalyse . . . . . . . . . . . . . 58

4.2.6. Numerische Simulation . . . . . . . . . . . . . . . . . . . 59

4.3. Partikelmodell . . . . . . . . . . . . . . . . . . . . . . 61

4.3.1. Lagrange-Formulierung . . . . . . . . . . . . . 62

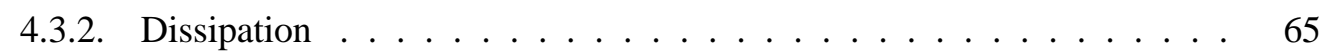

4.3.3. Äußere Kräfte . . . . . . . . . . . . . . . . . . . . . . . . 67

4.3.4. Translation ........................ 67

4.3.5. Wechselwirkung .................. . . 73

4.4. Vergleich der Modelle . . . . . . . . . . . . . . . . . . . . 75

4.5. Einfluß der Kompressibilität auf die Blasendynamik . . . . . . . . . . . . . . 77

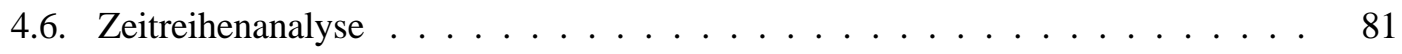


4.6.1. Phasenkohärenz . . . . . . . . . . . . . . . . . . . . . 82

4.6.2. Modell des Kavitationsblasenfeldes . . . . . . . . . . . . . . . . . . 84

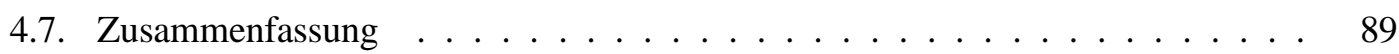

$\begin{array}{ll}\text { 5. Zusammenfassung } & 91\end{array}$

A. Particle-Tracking-Velocimetry-Toolbox 94

$\begin{array}{lr}\text { Literaturverzeichnis } & 98\end{array}$ 


\section{Einleitung}

Gegenstand dieser Arbeit ist die theoretische Beschreibung und experimentelle Untersuchung raum-zeitlicher Strukturbildung in akustischen Kavitationsblasenfeldern. Als Kavitation bezeichnet man das Entstehen von Hohlräumen in einer Flüssigkeit. Die Ursache von Kavitation ist das Anbringen von Spannungen in dem Medium oder der lokale Eintrag von Energie. Kavitation kann, wie in Abb. 1.1 dargestellt, nach ihrer Ursache klassifiziert werden. Der lokale Energieeintrag kann teilcheninduziert sein. Ein Beispiel hierfür sind Blasenkammern, die als Nachweisgeräte für radioaktive Strahlung verwendet werden. Ebenso kann ein optischer Durchbruch durch hohe Lichtintensität im Fluid und die damit verbundene Plasmabildung zum Entstehen von Blasen führen; ähnliches gilt für einen elektrischen Durchbruch. Bei hydrodynamischer Kavitation beruhen die sie erzeugenden Zugspannungen auf hohen Druckgradienten in der Strömung. Beispiele für hydrodynamische Kavitation sind in Abb. 1.2 dargestellt. Entstehen die Blasen in einem Schallfeld hoher Intensität, so wird dies als akustische Kavitation bezeichnet.

Ein Beispiel eines akustischen Kavitationsblasenfeldes ist in Abb. 1.3 gezeigt. Ein solches Blasenfeld besteht aus etwa $10^{3}$ bis $10^{4}$ beobachtbaren Blasen mit Ruheradien von typischerweise 5-10 $\mu \mathrm{m}$. Deutlich erkennbar ist die inhomogene Verteilung der Blasen in dendritischen oder filamentartigen Verästelungen (sog. streamer). Auf diesen Bahnen bewegen sich die Blasen aus den Randbereichen, wo sie entstehen, in den zentralen Bereich der Struktur, wo sie durch Kollision und Fragmentierung zerstört werden. Auf ihrem Weg wechselwirken die Blasen mit anderen Blasen, mit dem äußeren Schallfeld und der Bewegung der Flüssigkeit auf komplexe Weise. Die gesamte, aus einer Vielzahl von im Schallfeld schwingenden Blasen bestehende, dreidimensionale Struktur ist in ihrer Gestalt außerordentlich stabil. Wegen der Ähnlichkeit der Erscheinung von elektrischen Entladungsstrukturen mit den hier beobachteten Strukturen in akustischen Kavitationsblasenfeldern wurde für diese die Bezeichung "Akustische Lichtenberg-Figuren" vorgeschlagen.

Kavitationsphänomene sind in vielen technologischen und verfahrenstechnischen Anwendungen von zentraler Bedeutung. In vielen Anwendungen entfalten die Kavitationsblasen eine erhebliche zerstörerische Wirkung. So werden Schiffschrauben, Turbinen und Einspritzanlagen durch hydrodynamische Kavitation erodiert. In der Sonochemie hingegen wird die akustische Kavitation erfolgreich in Reaktoren eingesetzt, um chemische Reaktionen zu katalysieren. In verfahrenstechnischen Anlagen kommt es häufig zur Bildung von Mehrphasenströmungen, in denen flüssige und gasförmige Phasen koexistieren. Die Transporteigenschaften dieser hydrodynamischen Systeme werden wesentlich von dem Vorhandensein und der Wechselwirkung dieser verschiedenen Phasen beeinflußt. Es ist daher eine detaillierte Kenntnis der involvierten physikalischen Prozesse notwendig.

Motiviert durch die Breite der technischen Anwendungen und ihrer Bedeutung, hat sich die Kavitationsblasendynamik zu einem Gebiet intensiver Grundlagenforschung entwickelt. Insbesondere die Sonolumineszenz von einzelnen Blasen und Blasenfeldern hat in den letzten Jahren großes wissenschaftliches Interesse geweckt.

Akustische Kavitationsblasenfelder sind komplexe Mehrphasensysteme, die Phänomene der 


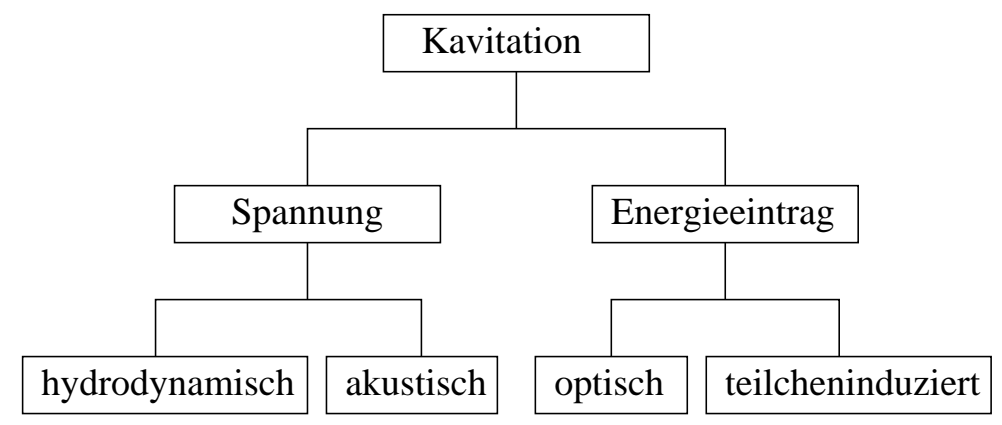

Abbildung 1.1.: Klassifikation von Kavitation nach Art des Energieeintrages in das Medium (Darstellung nach [46]).

raum-zeitlichen Strukturbildung aufweisen. Neben der technischen Bedeutung sind sie daher insbesondere wegen dieser nichtlinearen dynamischen Eigenschaften von großem wissenschaftlichem Interesse. Bereits eine einzelne Blase kann eine außerordentlich komplexe Schwingungsdynamik aufweisen. Ein räumlich ausgedehntes System von im Schallfeld miteinander wechselwirkenden Blasen besitzt die Fähigkeit zur Selbstorganisation.

Was bedeutet Selbstorganisation? Als Organisation bezeichnet man das funktionale Zusammenspiel der Elemente eines Systems zu einer das System betreffenden Wirkung [35]. Dies wird in der Regel von außen gesteuert. Unter Selbstorganisation versteht man die spontane, d.h. die ohne direkten äußeren Einfluß erfolgende Ausbildung eines höheren Grades des Zusammenwirkens der Elemente des Systems. Ein System ist eine relativ stabile, geordnete Gesamtheit von Elementen und ihren Beziehungen untereinander, die durch die Existenz bestimmter Gesetze charakterisiert ist. Die Struktur eines Systems ist die Art dieser Anordnungen und Verknüpfungen der Elemente.

Die Merkmale, mit denen diese Beziehungen der Teile, ihre Anordnung und Verknüpfung charakterisiert werden können, sind Raum- und Zeitskalen. Gemeint sind damit beispielsweise die typischen Abmessungen der einzelnen Objekte, ihr mittlerer Abstand oder eine charakteristische Länge, über die sie miteinander wechselwirken. Es können aber auch typische Relaxationszeiten der Bewegung der Objekte oder der räumlichen Anordnung, die sie gemeinsam bilden, als Charakterisierungsmerkmal dienen.

Als Strukturbildung bezeichnet man nun das Entstehen höherer, reicherer, geordneterer Strukturen. Von geordneteren Strukturen kann man sprechen, wenn sich die Symmetrie des Systems verringert hat: Strukturbildung ist verknüpft mit Symmetriebrechung. Selbstorganisation ist spontane Symmetriebrechung. Das Entstehen von Struktur ist verküpft mit der Instabilität eines Ausgangszustandes und dem Übergang in einen anderen stabilen Zustand. Diese Instabilität des Ausgangszustandes ist somit die grundlegende Voraussetzung für den Prozeß der Strukturbildung.

Es ist für die Charakterisierung des Gesamtsystems zunächst von Bedeutung, die Eigenschaften elementarer Objekte selbst zu kennen. In dem uns interessierenden Fall akustischer Kavitationsblasenfelder entspricht dies der Kenntnis der dynamischen Eigenschaften einer einzelnen Blase, d.h. ihrer Schwingung im Schallfeld und ihrer Bewegung durch die Flüssigkeit. Die beobachteten Strukturen ergeben sich dann als eine Eigenschaft des Systems, die durch die Wechselwirkung der Blasen untereinander und mit dem äußeren Schallfeld oder mit einer äußeren Strömung entstehen.

Das funktionale Zusammenwirken der einzelnen Komponenten des physikalischen Systems ist 

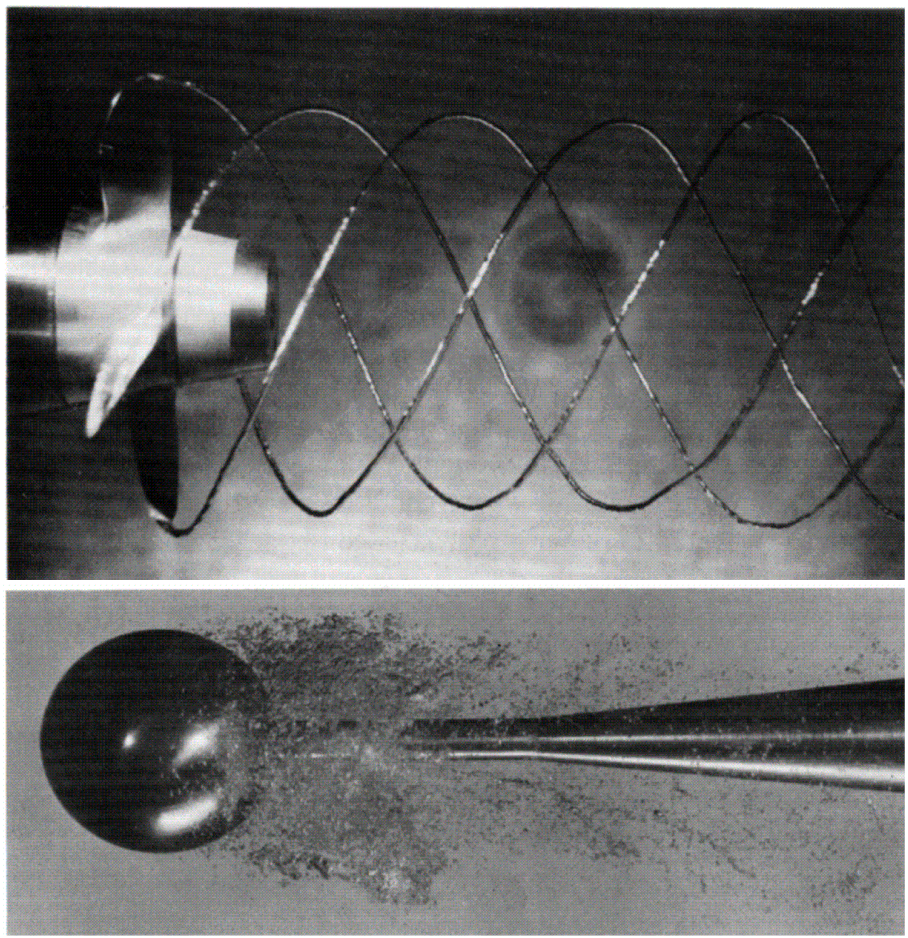

Abbildung 1.2.: Beispiele für hydrodynamische Kavitation [84].

in Abb. 1.4 dargestellt. Die Wirkung eines äußeren Schallfeldes besteht zunächst darin, daß es Blasen in der Flüssigkeit entstehen läßt. Die entstandenen Blasen werden durch den wechselnden Druck des Schallfeldes zu Schwingungen angeregt. Gleichzeitig wirkt auf die Blasen ein Schallstrahlungsdruck, der die Blasen im Schallfeld bewegt und so ihre Anordnung verändert. Die räumliche Verteilung der Blasen hat ihrerseits einen entscheidenden Einfluß auf das Schallfeld selbst durch die signifikante Abhängigkeit der Schallgeschwindigkeit von der lokalen Blasendichte. Eine Folge dieser Wechselbeziehung von Schallfeld und Blasenverteilung ist die Selbstkonzentration der Blasen und die Selbstfokussierung des Schallfeldes.

Die zu Schwingungen angeregten Blasen sind nun selbst wieder Quellen eines gestreuten Schallfeldes, durch welches sie miteinander wechselwirken. Ebenso wie beim äußeren Schallfeld ist auch bei dem gestreuten Schallfeld die Wirkung zweifach: Zum einen dient das Streufeld als zusätzliche Anregung und zur Kopplung der Blasenschwingungen, zum anderen wirken zwischen den Blasen Schallstrahlungskräfte, die als sekundäre Bjerknes-Kräfte bezeichnet werden. Die Natur dieser Wechselwirkung ist, insbesondere für nichtlineare Blasenschwingungen, außerordentlich komplex.

Bisher war nur von der Wechselwirkung des äußeren und des gestreuten Schallfeldes mit den Blasen die Rede. In gleicher Weise kann man die Wirkung einer äußeren Strömung diskutieren. Eine äußere Strömung führt zu einer Umverteilung der Blasen in der Flüssigkeit. Hierdurch werden die Transporteigenschaften der Strömung beeinflußt und der Charakter der Strömung bestimmt. Die sich bewegenden Blasen wechselwirken untereinander durch die von ihnen induzierte Strömung.

Schließlich kann das stehende Schallfeld auch zum Auftreten stationärer Wirbelströmungen in 


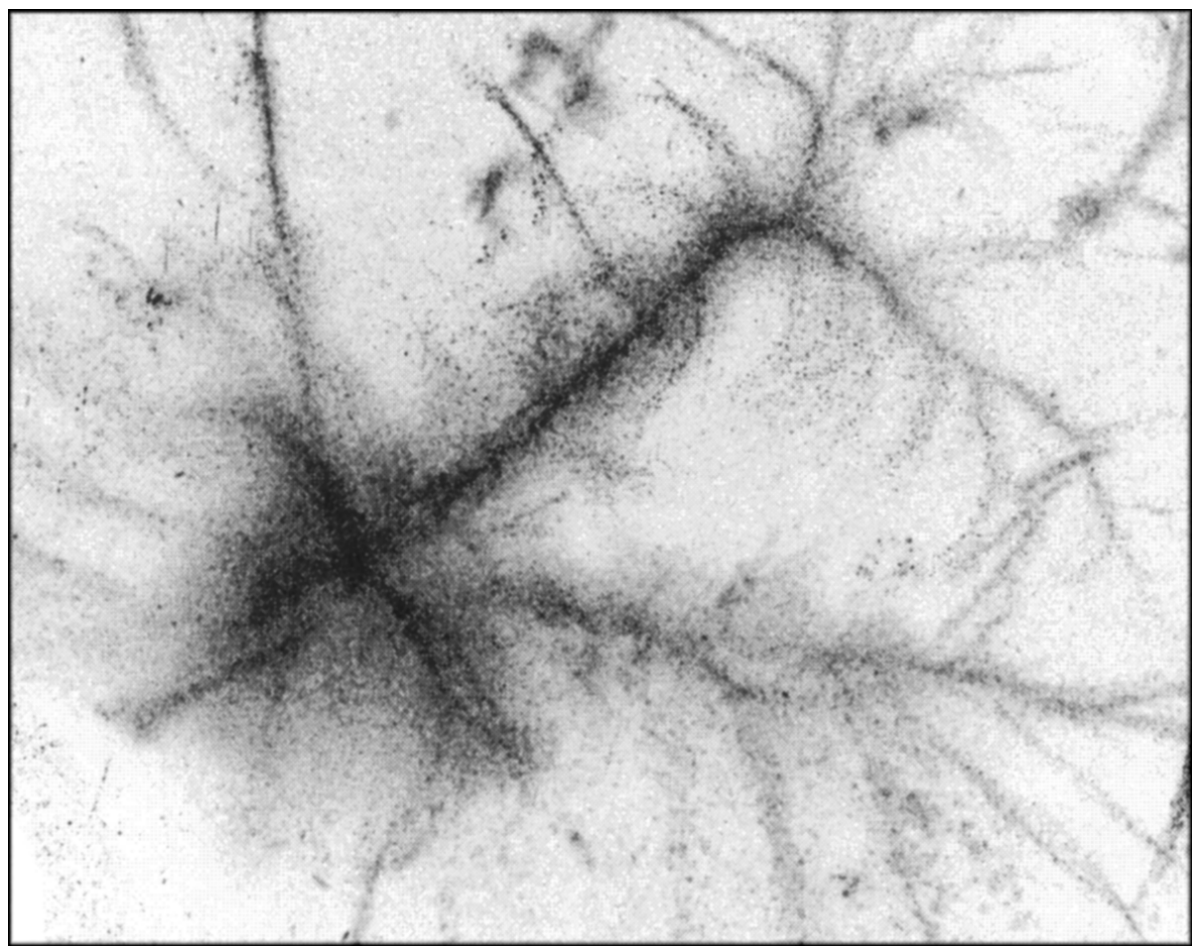

Abbildung 1.3.: Beispiel eines akustischen Kavitationsblasenfeldes.

dem Resonator führen. Diese Bewegung wird akustische Strömung genannt und ergibt sich als Folge des Einflusses der Zähigkeit auf die Schallwelle in zweiter Ordnung der Amplitude. Turbulente Geschwindigkeitsschwankungen können selbst wieder Schallquellen im umgebenden Flüssigkeitsvolumen sein. Auf diese Weise entsteht aus Schall Strömung und aus Strömung Schall.

Neben der funktionalen Beziehung der Elemente eines Systems - oder mit anderen Worten: ihrer Wechselwirkung miteinander - sind die räumlichen und zeitlichen Skalen der Bewegung und Anordnung der Blasen für die Charakterisierung des Systems von zentraler Bedeutung.

Die in dieser Arbeit diskutierten Phänomene werden im Rahmen der Hydrodynamik behandelt. Die Hydrodynamik beschreibt die Bewegung von Flüssigkeiten und Gasen und geht davon aus, daß diese makroskopischen Charakter haben und die Fluide daher als Kontinua angesehen werden können. Für jedes beliebig kleine Volumenelement $\delta \mathcal{V}$ der Flüssigkeit gelte, daß dessen charakteristische Ausdehnung $a=\delta \mathcal{V}^{1 / 3}$

- sehr klein gegenüber der charakteristischen Längenskala der betrachteten Bewegung der Flüssigkeit und

- sehr groß gegenüber der mittleren freien Weglänge der Moleküle sei.

Dies ist der Gegenstand der Kontinuumshypothese der Hydrodynamik. Diese Hypothese wird in dieser Arbeit durchgehend als erfüllt vorausgesetzt. Für alle im Folgenden eingeführten Skalen gelte, daß sie groß seien gegenüber $a$.

Das äußere Schallfeld oder die Geometrie eines Resonators liefert eine makroskopische Skala, auf der die langsame Drift der filamentartigen Strukturen mit bloßem Auge beobachtet werden kann. Jedes Filament hingegen besteht aus einer Vielzahl individueller Blasen. Durch 


\begin{tabular}{lll}
\multicolumn{3}{c}{ Raumskalen } \\
\hline mikro & Blasengleichgewichtsradius $R_{0}$ & $10^{-6} m$ \\
meso & mittlerer Blasenabstand & $10^{1}-10^{2} R_{0}$ \\
makro & Wellenlänge des Schallfeldes & $10^{4} R_{0}$ \\
& & \\
& Zeitskalen & \\
\hline schnell & Periode der Anregung $T$ & $10^{-4} s$ \\
mittel & Relaxationszeit der Blasendrift & $10^{2} T$ \\
langsam & Relaxationszeit der Streamerdrift & $10^{4} T$
\end{tabular}

Tabelle 1.1.: Charakterisierung von Raum- und Zeitskalen in akustischen Kavitationsblasenfeldern.

die einzelne Blase und die Periode der Anregung wird eine mikroskopische Raum- und eine schnelle Zeitskala festgelegt (vgl. Tabelle 1.1). Die räumliche Reorganisation der Blasen ist charakterisiert durch eine typische Relaxationszeit der Blasenbewegung und eine mesoskopische Raumskala. Die Größenordnung der genannten Skalen wird erweitert, wenn man die Dynamik des Blasenkollapses in die Betrachtung einbezieht. Ein akustisches Kavitationsblasenfeld ist daher ein hydrodynamisches Mehrphasensystem und Mehrskalensystem, welches in seiner raum-zeitlichen Dynamik Größenordnungen umspannt.

Die zentrale Fragestellung dieser Arbeit ist daher die Bedeutung der mikroskopischen Dynamik der Blasen auf schnellen Zeitskalen für das Entstehen und die Stabilität von makroskopischen Strukturen einer Vielzahl von individuellen Blasen auf langsamen Zeitskalen.

Beide Aspekte - die Wechselwirkung der Blasen untereinander und mit Schallfeld und Strömung sowie die involvierten charakteristischen Skalen - bilden den Ausgangspunkt und den roten Faden, an dem sich die Argumentation in dieser Arbeit entlang bewegt.

Die Intention war, grundlegende Information über das physikalische System zu sammeln und darauf aufbauend eine theoretische Beschreibung zu entwickeln. Daher bildet die Beobachtung, d.h. das Experiment, den Ausgangspunkt der Untersuchung. Ihr folgt die Theoriebildung. Dieser Entstehungsprozeß spiegelt sich im Aufbau der Arbeit wider, der im Folgenden dargestellt wird:

In Kapitel 2 werden die experimentellen Methoden zur Untersuchung der genannten Fragestellungen dargestellt. Die räumliche Struktur der Kavitationsblasenfelder wurde mit einer Hochgeschwindigkeitskamera untersucht und die Trajektorien der Blasen mit Hilfe der Particle Tracking Velocimetry (PTV) gemessen. Die hierzu verwendeten Algorithmen werden eingeführt und diskutiert. Es wurden Radien- und Druckmessungen durchgeführt. Die mit diesen Methoden gewonnenen Ergebnisse sind in Kapitel 3 zusammengefaßt. Dieses in der Arbeit zentrale Kapitel bildet den Übergang von der experimentellen Untersuchung zu der theoretischen Interpretation und Beschreibung in Kapitel 4. Die experimentellen Ergebnisse dienen dabei zum einen als Motivation und als Ausgangspunkt für die Auswahl und Entwicklung geeigneter theoretischer und numerischer Methoden. Zum anderen dienen sie im Verlauf der theoretischen Untersuchung dem Rückbezug und Vergleich.

In Kapitel 4 werden zwei Modelle zur Beschreibung der akustischen Blasenfelder betrachtet. Zum einen wird ein Kontinuumsmodell numerisch untersucht, zum anderen wird das Partikelmodell entwickelt und untersucht. Die beiden Modelle werden verglichen. Der Einfluß der Kom- 


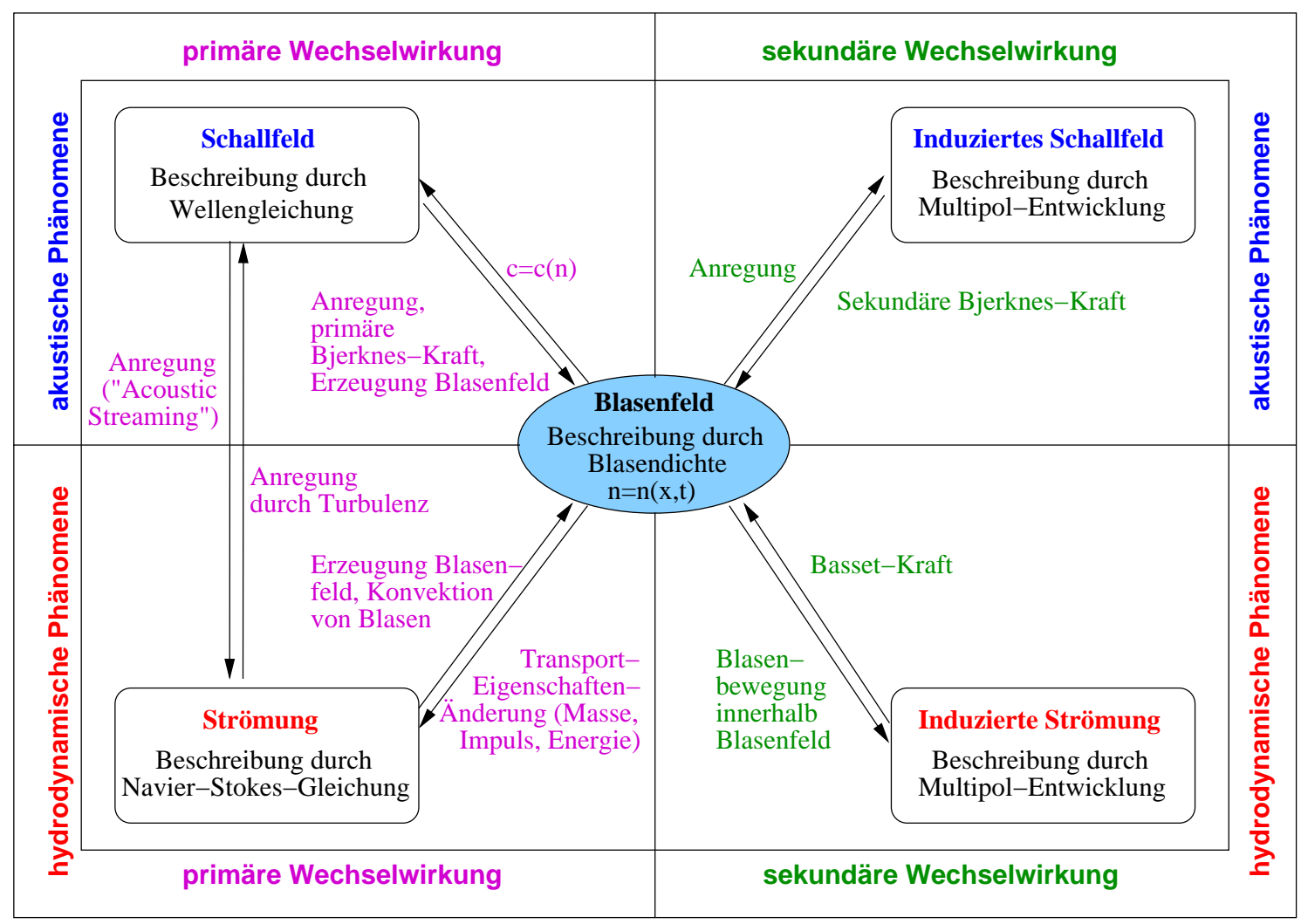

Abbildung 1.4.: Funktionale Gliederung der Wechselwirkungsprozesse.

pressibilität der Flüssigkeit auf die Blasendynamik wurde modelliert und diskutiert. Im Folgenden wird dann die grundlegende Fragestellung der Analyse der kollektiven Dynamik akustischer Kavitationsblasenfelder mit Hilfe der Methoden der nichtlinearen Zeitreihenanalyse aufgegriffen. Zum Abschluß des Theorie-Kapitels wird eine Zusammenfasssung gegeben. Detailinformationen, die die Darstellung in den Kapiteln unnötig belasten würden, aber für das tiefere Verständnis und insbesondere für die Reproduzierbarkeit der dargestellten Ergebnisse dringend notwendig sind, wurden aus den jeweiligen Kapiteln ausgelagert und im Anhang am Schluß der Arbeit untergebracht. Der Hauptteil der Arbeit schließt mit der Zusammenfassung und dem Ausblick. 


\section{Experiment: Meßmethoden}

In diesem Kapitel werden die experimentellen Methoden vorgestellt und diskutiert, mit Hilfe derer die raum-zeitliche Struktur akustischer Kavitationsblasenfelder untersucht wird. Es sollen die räumliche Verteilung der Blasen, ihre Geschwindigkeiten und ihre Radien gemessen und über Bildsequenzen verfolgt werden.

In Abschn. 2.1 wird zunächst eine Übersicht über die Meßverfahren der Strömungsmechanik gegeben und die Auswahl des gewählten bildgebenden Verfahrens begründet. Das für die experimentellen Untersuchungen in dieser Arbeit grundlegende Meßverfahren ist die ParticleTracking-Velocimetry. Das Flußdiagramm dieses Verfahrens ist die Grundlage für die Struktur dieses Kapitels. Diesem Flußdiagramm entspricht eine Hierarchie von Bildverarbeitungsoperationen von der Bildaufnahme bis zum Bildverstehen, d.h. dem Verständnis der beobachteten Dynamik.

Der experimentelle Aufbau wird in Abschn. 2.2 vorgestellt. Die Komponenten des Bildaufnahmesystems werden in Abschn. 2.3 vorgestellt und die mit der Bildaufnahme verbundenen Fehlerquellen diskutiert.

Die für die Bildbearbeitung grundlegenden Verfahren und ihre Implementierung werden in Abschn. 2.4 vorgestellt. Bildverarbeitungsverfahren können unterschieden werden in Verfahren, die objektbezogen sind und in solche, die grauwertorientiert sind. Beide Verfahren haben neben der Bildbearbeitung eine besondere Bedeutung in der Bewegungsanalyse, wie sie im Rahmen dieser Arbeit verwendet wird.

Der Übergang vom Einzelbild zur Bildsequenz erlaubt, die dynamische Entwicklung des beobachteten Systems zu verfolgen. Für räumlich ausgedehnte, dreidimensionale Systeme bedeutet die mit der üblichen Bildaufnahme verbundene Projektion auf die Bildebene einen wesentlichen Verlust von Information. Diese Information kann durch stereoskopische Verfahren wiedergewonnen werden. Die verwendeten Bildanalyseverfahren werden in Abschn. 2.5 vorgestellt.

Desweiteren wird in Abschn. 2.6 ein Verfahren zur optischen Radienmessung entwickelt und die Umsetzung dargestellt. Das Kapitel schließt mit einer Zusammenfassung.

\section{1. Übersicht über Meßverfahren in der Strömungsmechanik}

Die Abb. 2.1 zeigt eine Übersicht über die wichtigsten Verfahren der Geschwindigkeitsfeldmessung in der Strömungsmechanik. Für eine ausführliche Darstellung siehe beispielsweise [22]. Eine Charakterisierung der Verfahren ist durch die Dimension des Beobachtungsvolumens möglich. Punktmeßverfahren bestimmen lokal eine oder mehrere Komponenten des Geschwindigkeitsfeldes. Zu diesen Verfahren zählen Hitzdraht-Anemometrie, Laser-DopplerAnemometrie (LDA) und akustische Doppler-Anemometrie. Im Unterschied zur Hitzdraht-Anemometrie sind die beide letztgenannten Verfahren nicht invasiv. Vorteile der Methoden sind hohe 


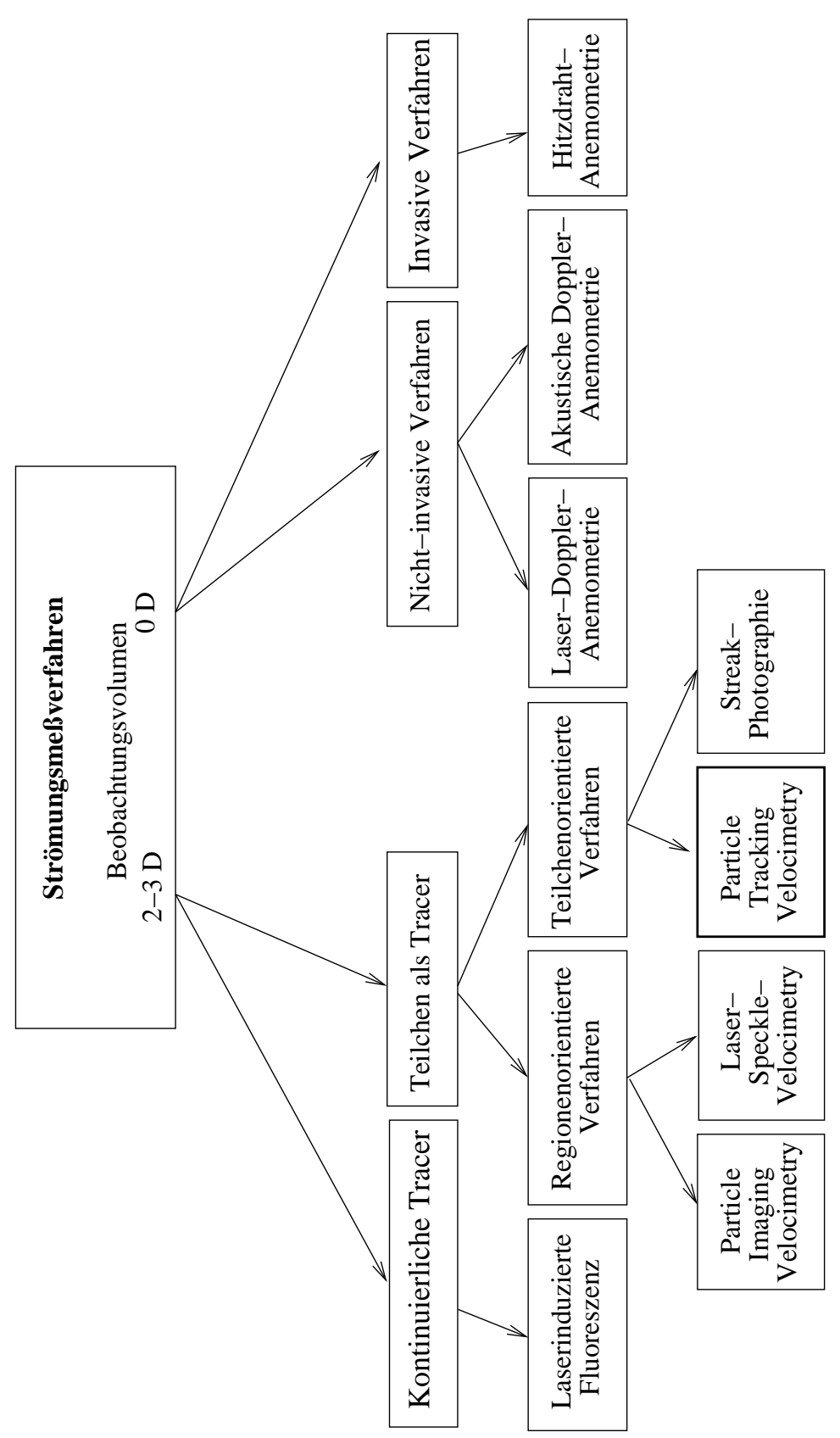

Abbildung 2.1.: Übersicht über die wichtigsten Strömungsmeßverfahren (Darstellung nach [29]; siehe auch [22]) 


\begin{tabular}{lcccc} 
& Hitzdraht & LDA & PIV & PTV \\
\hline räumliche Auflösung & niedrig & niedrig & sehr hoch & hoch \\
zeitliche Auflösung & hoch & sehr hoch & sehr niedrig & niedrig \\
Geschwindigkeitsbereich & hoch & sehr hoch & sehr hoch & niedrig \\
Dim. des Beobachtungsvolumens & 0 & 0 & 2 & $2-3$ \\
Dim. des Geschwindigkeitsfeldes & $1-3$ & $1-3$ & 2 & $2-3$ \\
Ergebnis & Vektoren & Vektoren & Vektoren & Trajektorien
\end{tabular}

Tabelle 2.1.: Vergleich ausgewählter Meßverfahren der Strömungsmechanik.

bzw. sehr hohe zeitliche Auflösung und Geschwindigkeitsdynamik. Die räumliche Auflösung ist jedoch niedrig, allerdings ist eine dreidimensionale Abtastung für stationäre Strömungen möglich.

Flächen- bzw. Volumenmeßverfahren haben ein zwei- bzw. dreidimensionales Beobachtungsvolumen. Kennzeichnend für diese Verfahren ist das Markieren der Strömung mit teilchenartigen oder kontinuierlichen passiven Tracern bzw. Markern, die mit der Strömung mitgenommen werden und deren Bewegung gemessen wird. Ein Beispiel für die Verwendung kontinuierlicher Tracer ist die einfache Strömungsvisualisierung durch das Einbringen von Streichlinien. Die Verwendung fluoreszierender Farbstoffe hierfür erlaubt das reversible Markieren der Strömung. Hierauf beruht die Laser-induzierte Fluoreszenz (LIF).

Werden feste Partikel verwendet, so können regionen- und teilchenorientierte Verfahren unterschieden werden. Die Particle-Image-Velocimetry (PIV) und Laser-Speckle-Velocimetry (LSV) sind regionenorientierte Methoden, da sie das Geschwindigkeitsfeld durch Mittelung der Geschwindigkeiten der Tracer einer Region bestimmen. Das Ziel teilchenorientierter Verfahren hingegen ist, die Bewegung einzelner Partikel aufzulösen. Beispiele hierfür sind StreakPhotographie und Particle-Tracking-Velocimetry (PTV). PIV und PTV unterscheiden sich durch den mittleren Teilchenabstand: Um die Bewegung einzelner Teilchen über eine Bildsequenz verfolgen zu können, muß der mittlere Teilchenabstand klein sein gegenüber dem Weg, den die Teilchen zwischen zwei aufeinander folgenden Aufnahmen zurücklegen. Die Tracer-Verfahren sind nicht-invasiv, sofern die Rückwirkung der Tracer auf die Strömung vernachlässigt werden kann [82].

Die genannten regionen- oder teilchenorientierten Verfahren erlauben zunächst nur die Messung eines zweidimensionalen Geschwindigkeitsfeldes. Um eine dreidimensionale Strömung zu erfassen, wurden holographische oder stereoskopische Verfahren entwickelt. Ein Beispiel hierfür ist Holographie-PIV. Es ist desweiteren möglich, Kombinationen der Verfahren zu verwenden, z.B. PIV und LIF. Die Eigenschaften ausgewählter Meßverfahren sind in Tabelle 2.1 zusammengefaßt.

Die Verfahren der Strömungsmechanik mit zwei- oder dreidimensionalem Beobachtungsvolumen basieren auf einem Bildverarbeitungssystem und sind daher wesentlich durch dessen Leistungsfähigkeit begrenzt. Ziel des Experimentes ist die Untersuchung der Bewegung der Kavitationsblasen. Hierbei ist sowohl die Dynamik einzelner Blasen als auch die Bewegung von Blasenwolken von Interesse. Daher wird als Meßverfahren die Particle Tracking Velocimetry gewählt, die es erlaubt, die Trajektorien einzelner Blasen zu verfolgen. 
Particle Tracking Velocimetry Die Projektion einer dreidimensionalen Anordnung von Objekten auf eine zweidimensionalen Ebene bedeutet Informationsverlust. Daher haben stereoskopische oder holographische Aufnahmetechniken das Ziel, diese Information wieder verfügbar zu machen $[65,8]$. Die Particle Tracking Velocimetry erlaubt in Verbindung mit stereoskopischen Aufnahmetechniken die dreidimensionale Rekonstruktion des Geschwindigkeitsfeldes.

In Abb. 2.2 ist ein Flußdiagramm für die Durchführung der PTV-Geschwindigkeitsfeldmessung gezeigt. Ausgangspunkt ist die Aufnahme einer Bildsequenz der Strömung. Die Bilddaten werden in einem ersten Verarbeitungsschritt aufbereitet, d.h. von Rauschen und inhomogener Beleuchtung befreit. Im nächsten Schritt werden die Objekte, d.h. die Tracer-Partikel bzw. die Blasen, in den Bilderen lokalisiert und mit Hilfe von Formparametern charakterisiert. Nach der Klassifikation der Objekte im Merkmalsraum im Rahmen der Bildanalyse, müssen - im Falle einer dreidimensionalen Analyse - nun korrespondierende Objekte in den Stereobildern gefunden werden und für diese die dreidimensionalen Objektkoordinaten rekonstruiert werden. Das eigentliche Ziel der PTV ist das Auffinden von Trajektorien, d.h. die Zuordnung und die Verfolgung von Objekten in einer Bildsequenz. In den weiteren Schritten können durch Interpolation und Visualisierung die Trajektorien dargestellt werden. Der folgende Aufbau dieses Kapitels orientiert sich an der Abfolge der Schritte in dem in Abb. 2.2 gezeigten Flußdiagramm.

\subsection{Experimenteller Aufbau}

In diesem Abschnitt wird eine Übersicht über die experimentellen Aufbauten gegeben, mit denen die Messungen durchgeführt wurden. Die Aufbauten können unterschieden werden in solche, die eine zwei- oder dreidimensionale Messung erlauben. Die Aufnahmen können jeweils im

- Durchlicht oder im

- Streulicht

erfolgen. Streulichtmessungen können weiter unterschieden werden nach der Geometrie der Beleuchtung, d.h. in zweidimensionale Lichtschnitte oder Lichtzylinder.

Die akustischen Kavitationsblasenfelder werden in einem quaderförmigen Resonator erzeugt, an dessen Boden sich ein piezo-keramischer Schallwandler befindet. Bei Durchlichtmessungen befinden sich Lichtquelle und Kamera auf der optischen Achse. Ein Objekt streut Licht aus dem Strahlengang heraus und erscheint daher auf der Abbildung als Bereich verminderter Helligkeit. In Streulichtmessungen wird mit einem Lichtschnitt oder mit einem Lichtzylinder beleuchtet und die Kamera senkrecht zur Beleuchtungsrichtung plaziert. Die Objekte streuen Licht in die Kamera und erscheinen daher als Bereiche heller Grauwerte vor einem dunklen Hintergrund. Wird für die Streulichtaufnahmen eine nicht-gepulste Lichtquelle verwendet, so erscheinen bewegte Objekte als heller Streifen auf der Aufnahme. Die Gestalt dieses Streifens kann verwendet werden, um Geschwindigkeit und Beschleunigung des Objektes zu bestimmen. Stroboskopische Aufnahmen sind im Durchlicht mit gepulsten Lichtquellen oder mit einem triggerbaren elektronischen Verschluß der Kamera möglich. Eine stroboskopische Belichtung im Durchlicht reduziert allerdings schnell das Signal-Rauschverhältnis der Aufnahme und ist daher in der Regel ungeeignet. Die optischen Achsen werden nach Möglichkeit senkrecht zu den Wänden der Küvette gewählt, so daß Verzeichnungen durch Brechung an den Grenzflächen radialsymmetrisch sind. Werden im wesentlichen nur axiale Strahlen abgebildet und befindet sich das Meßvolumen nicht zu weit von der Grenzfläche entfernt, so kann Brechung i.d.R. vernachlässigt werden. Für Streulichtaufnahmen ist die Intensität der für die Durchlichtaufnahmen 


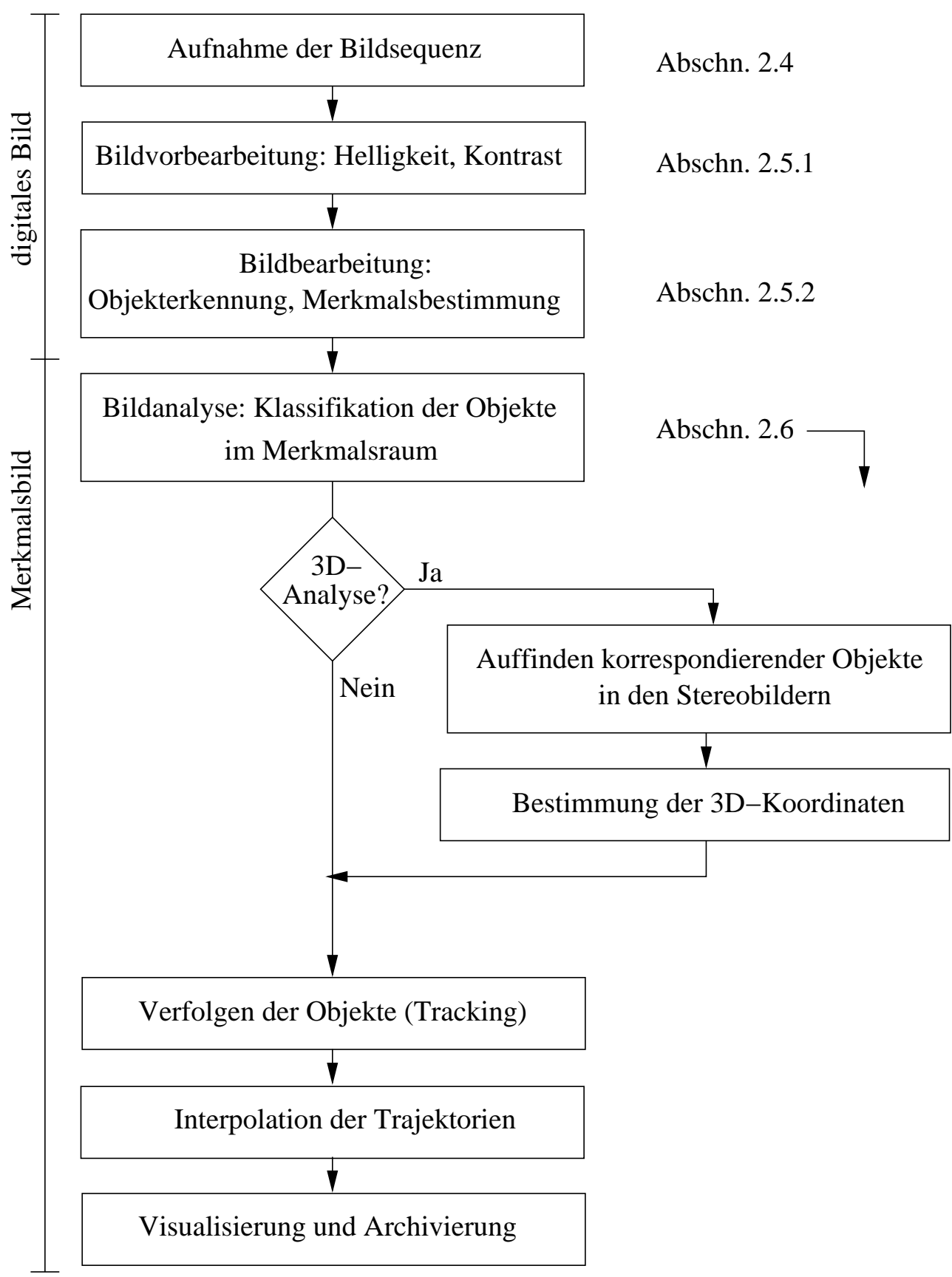

Abbildung 2.2.: Flußdiagramm der Particle Tracking Velocimetry (PTV). (Vgl. hierzu auch [65].) 


\section{LED-Array Küvette CCD-Sensor}

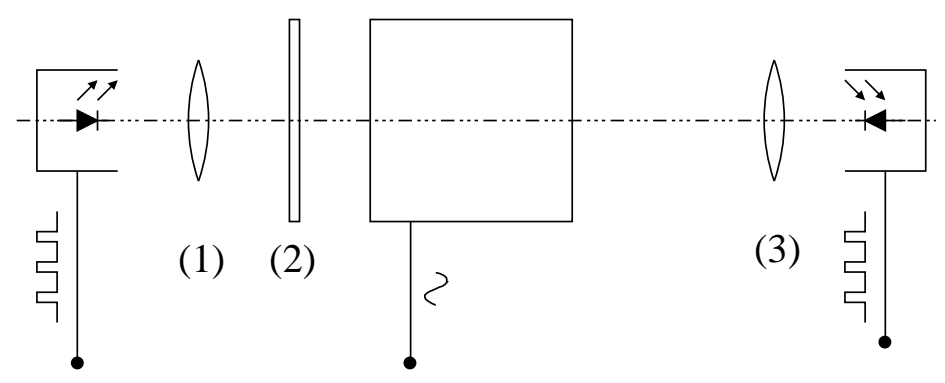

Abbildung 2.3.: Prinzipdarstellung des Aufbaus für die Durchlichtaufnahme in Aufsicht. Als Lichtquelle wird ein LED-Feld verwendet. Es bezeichnen (1) die Beleuchtungsoptik, (2) Streuscheibe und (3) Abbildungsoptik.

verwendeten LED-Felder nicht ausreichend. Es werden daher hierfür Bogen- oder Halogenlampen verwendet. Die Intensität des gestreuten Lichtes ist abhängig von der Größe der Objekte und vom Beobachtungswinkel relativ zur Einfallsrichtung. Die Streuamplitude ist im Winkel von $90^{\circ}$ bezogen auf die Richtung des einfallenden Lichtes um Größenordnungen kleiner als in Vorwärtsrichtung.

In Abb. 2.3 ist eine Prinzipdarstellung der Aufsicht des experimentellen Aufbaus dargestellt, wie er in dieser Arbeit für Durchlichtaufnahmen verwendet wird. Die Küvette hat die Abmessungen $5 \times 5 \times 10 \mathrm{~cm}^{3}$. Das Beobachtungsvolumen ist typischerweise $1 \times 1 \times 1 \mathrm{~cm}^{3}$. Die Beleuchtung erfolgte bei allen Durchlichtaufnahmen mit einem gepulsten LED-Feld. Die Beleuchtungssoptik und die Streuscheibe dienen zur gleichmäßigen Ausleuchtung des Bildfeldes. Das Bild wird mit der Abbildungsoptik auf die Sensoren der CCD-Kamera abgebildet. Je nach verwendetem Trigger-Modus kann die Kamera freilaufend betrieben oder phasenstarr an die Anregung gekoppelt werden. Bei den Aufnahmen im Durchlicht ist der Verschluß der Kamera zwischen zwei Bildern geöffnet und die Belichtung erfolgt mit einem Lichtpuls von etwa $3 \mu$ s Dauer.

Der Aufbau für stereoskopische Aufnahmen ist in Abb. 2.4 dargestellt. Es handelt sich hierbei um zwei Beleuchtung-Kamera-Einheiten entsprechend Abb. 2.3. Es werden zwei zueinander orthogonale Ansichten des Beobachtungsvolumens betrachtet. Der Aufbau muß zur Rekonstruktion der Objektpositionen kalibriert werden. Beide Kameras können über eine MehrkanalBildspeicherkarte ausgelesen werden. Hierzu müssen sie allerdings synchronisiert werden.

Abb. 2.5 zeigt einen alternativen Aufbau für stereoskopische Aufnahmen, sofern nur eine Kamera zur Aufzeichnung verwendet wird. Der Grundaufbau entspricht Abb. 2.3, jedoch wird der Strahlengang durch Spiegel und Prismen auf einen CCD-Chip abgebildet. Auf den beiden Hälften werden die orthogonalen Bilder des Beobachtungsvolumens abgebildet. Es entfällt eine aufwendige Synchronisation der Kameras, wie dies bei Aufbauten mit zwei Kameras notwendig ist. Weiterhin ist nur die Kalibrierung einer Kamera notwendig. Ein Nachteil dieser Aufnahmetechnik ist, daß sich die räumliche Auflösung halbiert. Insbesondere für Hochgeschwindigkeitsaufnahmen kann dies bei der ohnehin reduzierten Auflösung problematisch sein. Nachteilig ist ebenfalls die Vignettierung durch die sich vor der Abbildungsoptik befindlichen Spiegel bzw. Prismen.

In Abb. 2.6 (a) ist der Aufbau für zweidimensionale Streulichtaufnahmen gezeigt. Zur Beleuchtung wird eine Weißlichtquelle verwendet. Mit Hilfe einer Lichtschnittoptik kann ein ebener Lichtschnitt erzeugt werden. Die Aufnahme erfolgt senkrecht zur Beleuchtungsrichtung. Die Intensität des Streulichtes kann jedoch erheblich erhöht werden, wenn in Vorwärtsrichtung um 


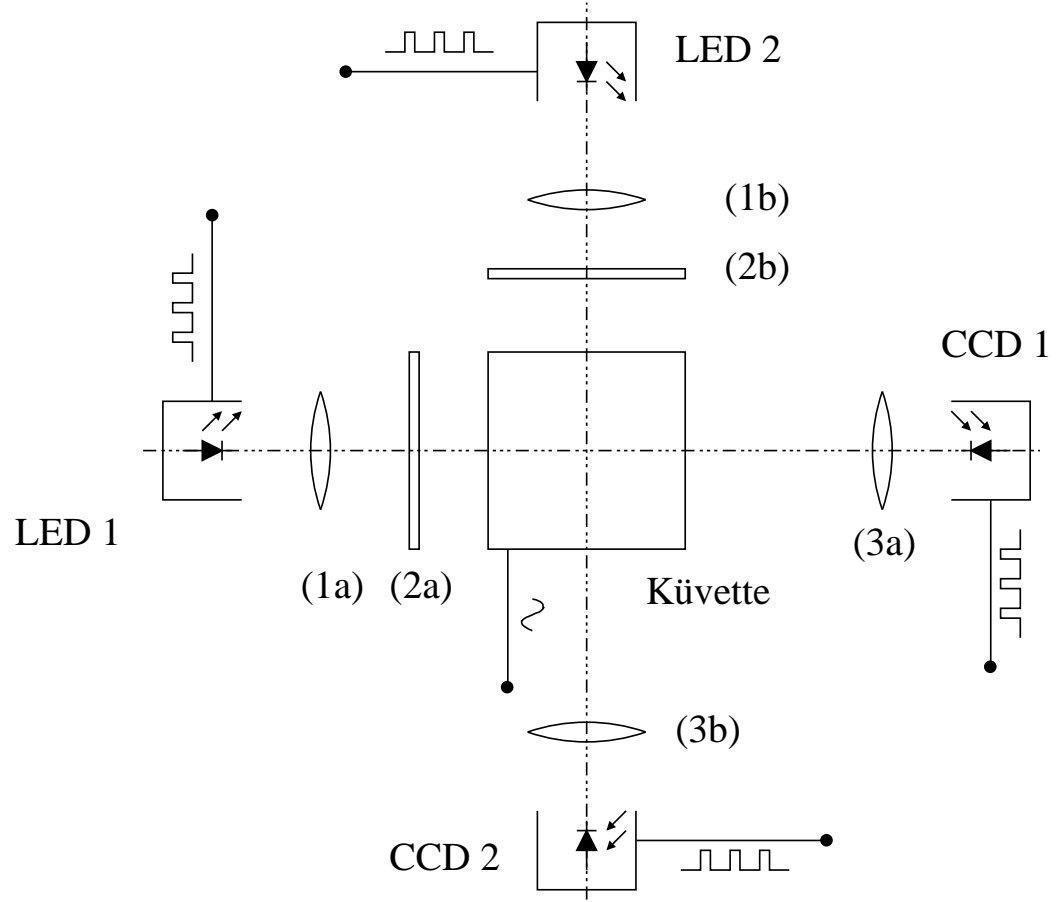

Abbildung 2.4.: Prinzipdarstellung des Aufbaus für die stereoskopische Durchlichtaufnahme in Aufsicht. Als Lichtquelle werden zwei LED-Felder verwendet. Es bezeichnen jeweils (1a,b) die Beleuchtungsoptiken, (2a,b) die Streuscheiben und (3a,b) die Abbildungsoptiken. Der Aufbau ist symmetrisch in der Art und den Eigenschaften der gewählten Komponenten.

einen kleinen Winkel $\delta$ gedreht wird. Der Lichtschnitt kann transversal um die Strecke $\delta x$ durch das Meßvolumen bewegt werden. In Abb. 2.6 (c) ist die Winkelabhängigkeit der Streulichtamplitude an einem Beispiel eines Partikels mit einem Durchmesser von $1 \mu \mathrm{m}$ gezeigt. Die Amplitude des gestreuten Lichtes ist hier logarithmisch aufgetragen und wächst in Vorwärtsrichtung um Größenordnungen an.

Der Aufbau für die Beobachtung eines Volumens im Streulicht ist nicht in einer Abbildung gezeigt. In dieser Anordnung muß der Lichtzylinder von oben in die Küvette hinein scheinen. Die Kameras sind dann wie in Abb. 2.4 orthogonal angeordnet.

Im folgenden Abschnitt werden die Komponenten des Bildbearbeitungssystems vorgestellt.

\subsection{Das Bildaufnahmesystem}

In diesem Abschnitt werden die Komponenten des Bildaufnahmesystems vorgestellt. Ein Bildaufnahmesystem kann aufgeteilt werden in die Komponenten der Hardware und ihre Funktionen. Zu den Hardware-Komponenten zählen Beleuchtung, optische Komponenten, der Bildsensor und die Elektronik der Kamera sowie der Bildspeicher. Diese Komponenten lassen sich nach funktionalen Gesichtspunkten zu Abbildung, Digitalisierung und Quantisierung zuammenfassen (Tabelle 2.2). Mit jeder einzelnen Komponente sind Fehler verbunden, die im Folgenden diskutiert werden. Die Verwendung des Bildaufnahmesystems für Meßzwecke erfordert eine Kalibrierung und Fehlerkorrektur. Diese kann auf der Grundlage eines Modells des Abbildung- 


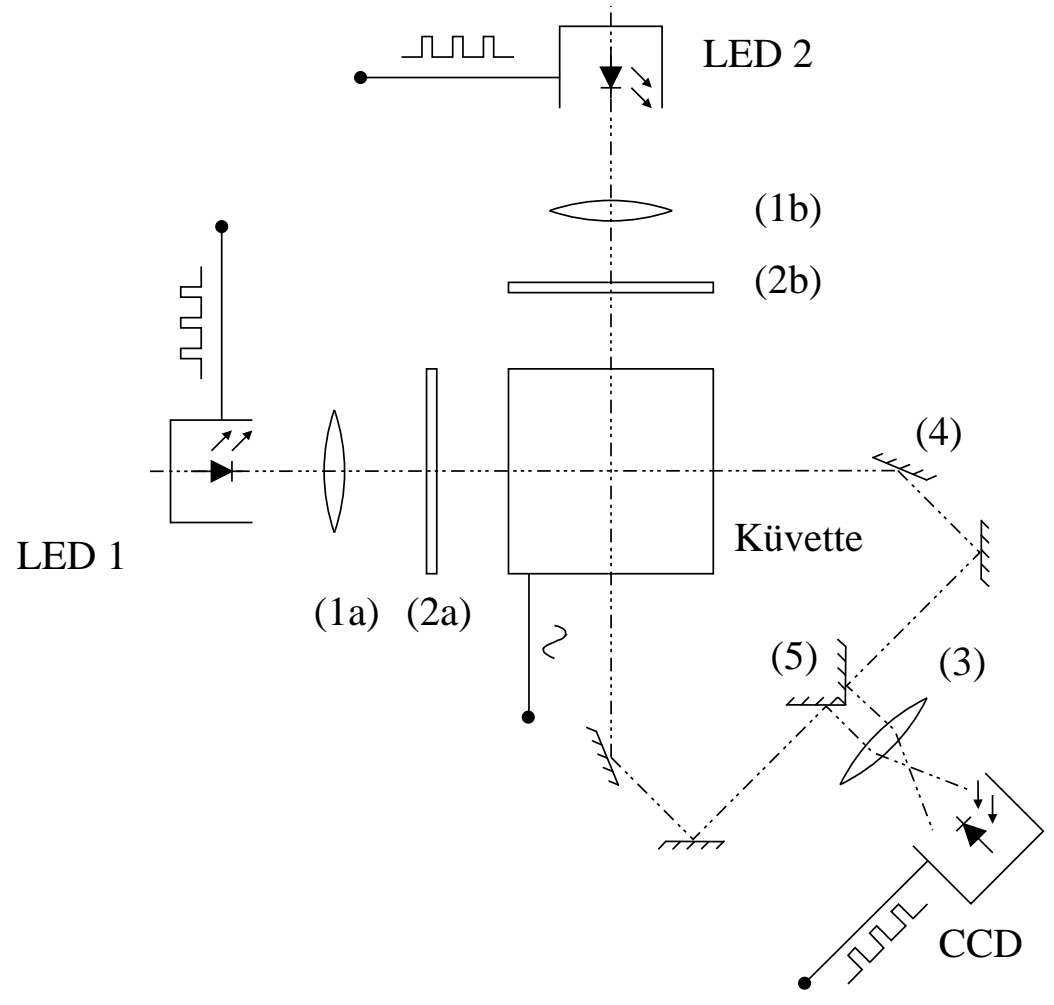

Abbildung 2.5.: Prinzipdarstellung des Aufbaus für die stereoskopische Durchlichtaufnahme in Aufsicht. Als Lichtquelle werden zwei LED-Felder verwendet. Es bezeichnen jeweils $(1 a, b)$ die Beleuchtungsoptiken, $(2 a, b)$ die Streuscheiben und (3) die Abbildungsoptiken. Die Strahlen werden umgelenkt mit den Spiegeln (4) und den Prismen (5).

ssystems durchgeführt werden, das in Abschn. 2.3.3 beschrieben wird.

\subsubsection{Hardware-Komponenten}

Beleuchtung Die Lichtquellen, mit denen die Objekte beleuchtet werden, können charakterisiert werden durch ihre spektralen Eigenschaften und ihre Intensität. Für stroboskopische Beleuchtung ist die Pulsform und -dauer sowie die maximale Repetitionsrate von Bedeutung. Fehler entstehen durch räumlich inhomogene Beleuchtung der Bildebene oder zeitliche Schwankungen in der Intensität.

Optik Die optischen Komponenten bilden das Objekt auf der Bildebene ab. Dies kann im Rahmen der geometrischen Optik beschrieben werden. Neben der beugungsbegrenzten Auflösung entstehen Abbildungfehler durch Linsenfehler (Aberrationen), insbesondere tangentiale und radiale Linsenverzeichnung. Diese Abbildungsfehler können durch eine geeignete Kalibrierung kompensiert werden. Hierdurch ist es möglich, konventionelle Objektive für Meßzwecke einzusetzten. Eine unscharfe Abbildung eines Objektes kann durch Defokussierung entstehen oder als Folge der Bewegung des Objektes (Bewegungsunschärfe). 
(a)

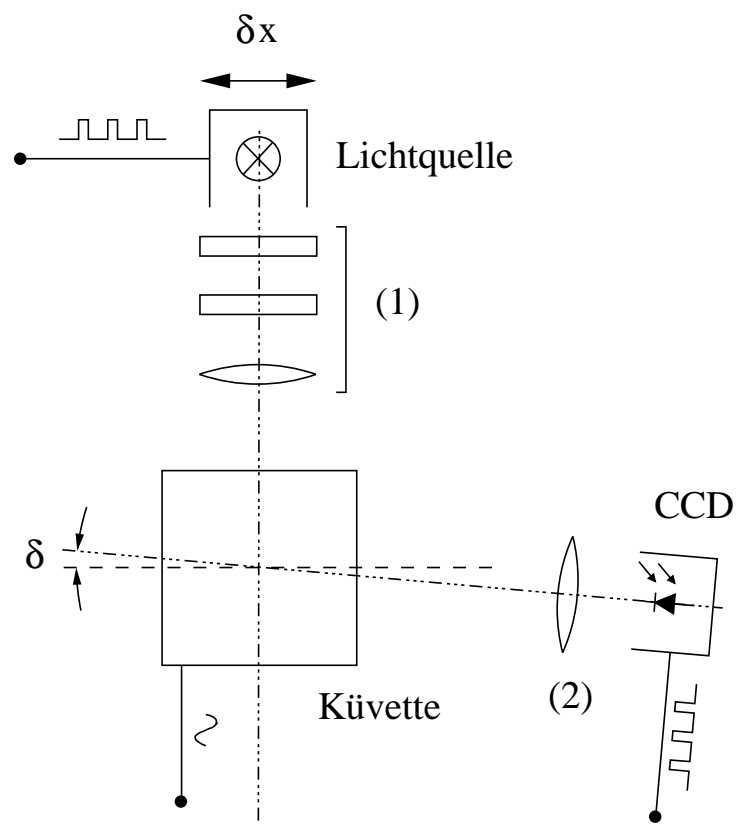

(b) Lichtschnittoptik, Seitenansicht

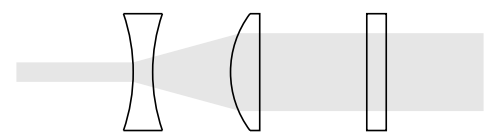

(c) Streulicht, Partikel $1 \mu \mathrm{m}$

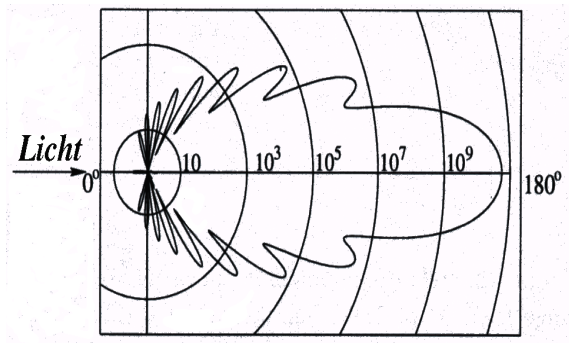

Abbildung 2.6.: (a) Schematische Darstellung des Aufbaus für Aufnahmen mit Lichtschnitt (Darstellung in Aufsicht). (1) Lichtschnittoptik und (2) Abbildungsoptik. Die Lage des Lichtschnittes kann durch Variation von $\delta x$ variiert werden. Die Streuamplituden wachsen erheblich, wenn in Vorwärtsrichtung mit dem Winkel $\delta$ beobachtet wird. (b) Lichtschnittoptik aus (a) in Seitenansicht. (c) Illustration der Winkelabhängigkeit der Streuamplitude. Die Steulichtamplitude ist logarithmisch aufgetragen.

Bildsensoren Als Bildsensoren werden CCDs (harge $\underline{\text { Coupled }}$ Devices) verwendet. CCDs sind ein- oder zweidimensionale Anordnungen von Photodioden. Typische Kantenlängen der einzelnen Sensoren sind $1 \mu \mathrm{m}$ bis $10 \mu \mathrm{m}$. Auf einem Chip werden je nach Modell $256 \times 256$ oder $640 \times 480$ Elemente zusammengefaßt. Hochauflösende CCDs enthalten $5120 \times 5120$ Elemente. Typische Formate der CCD-Chips haben eine Diagonale von 6, 8 oder $11 \mathrm{~mm}$. Die einzelnen Elemente werden mit vertikalen und horizontalen Schieberegistern ausgelesen. Die CCDs können charakterisiert werden durch ihre spektrale Empfindlichkeit. Eine wesentliche Störquelle der Bildsensoren ist das Dunkelstromrauschen, welches empfindlich von der Temperatur abhängt. Das von der CCD-Kamera ausgegebene Signal kann, je nach Bauart, ein digitales oder analoges Videosignal sein.

Bildspeicher Der Bildspeicher, bzw. der sogenannte Framegrabber, wandelt das analoge Bildsignal der Kamera und bildet es in den Speicher ab. Zeilenjitter wird durch Synchronisationsprobleme bei schlechtem oder fehlangepaßtem PLL (phase locked loop) des Framegrabbers verursacht. Die Bandbreite des Framegrabbers wird limitiert durch die Bandbreite des peripheren Bussystems. Derzeit ist eine Übertragungsrate von etwa $132 \mathrm{MB} / \mathrm{s}$ möglich. 


\begin{tabular}{|l|l|l|}
\hline Komponente & Prozeß & \multicolumn{1}{|c|}{ Fehler } \\
\cline { 1 - 2 } Beleuchtung & Abbildung & $\begin{array}{l}\text { inhomogene Beleuchtung } \\
\text { Verzeichnungen }\end{array}$ \\
\cline { 1 - 3 } Optik & Digitalisierung & Dunkelstromrauschen \\
\cline { 1 - 3 } Bildsensoren & Quantisierung & $\begin{array}{l}\text { Quantisierungsrauschen } \\
\text { Zeilenjitter }\end{array}$ \\
\cline { 1 - 3 } Bildspeicher & & \\
\hline
\end{tabular}

Tabelle 2.2.: Komponenten, Prozesse und Fehlerquellen der Bildbearbeitung.

\subsubsection{Funktion}

Abbildung Die Bilderzeugung kann durch die Faltung des Urbildes mit der Punktantwort des optischen Systems beschrieben werden:

$$
\begin{aligned}
g(\mathbf{x})=\int_{-\infty}^{\infty} g^{\prime}\left(\mathbf{x}^{\prime}\right) h\left(\mathbf{x}-\mathbf{x}^{\prime}\right) \mathrm{d}^{2} \mathbf{x}^{\prime}=\left(g^{\prime} * h\right)(\mathbf{x}) & \\
& \mathcal{i} \mathcal{F} \\
\hat{g}(\mathbf{k})= & \hat{g}^{\prime}(\mathbf{k}) \hat{h}(\mathbf{k})
\end{aligned}
$$

wobei $h(\mathbf{x})$ die Punktantwort (PSF) und $\hat{h}(\mathbf{k})$ die optische Transferfunktion (OTF) bezeichnen. $g^{\prime}(\mathbf{x})$ kann hierbei als Grauwertbild betrachtet werden, das aus einem perfekten Sensor resultieren würde, d.h. einem optischen System (einschl. Sensor), dessen OTF identisch eins und dessen Punktantwort eine $\delta$-Funktion ist.

Digitalisierung Das auf die Fläche des Sensors abgebildete Bild wird nun digitalisiert. Hierzu muß das Bild, das repräsentiert ist durch eine kontinuierliche Grauwertverteilung $g(\mathbf{x}) \in \mathbb{R}^{2}$, auf eine endliche Anzahl diskreter Sensoren abgebildet werden. Die Sensoren seien durch eine Matrix $G_{m, n}$ mit $m, n \in \mathbb{Z}$ beschrieben. Die Digitalisierung kann daher als Abbildung

$$
\begin{array}{ccc}
D: g(\mathbf{x}) & \longrightarrow & G_{m, n} \\
\mathbf{x} \in \mathbb{R}^{2} & \longmapsto & m, n \in \mathbb{Z}
\end{array}
$$

formuliert werden. Der Prozeß der Digitalisierung kann in Abtastung und in die Begrenzung des Bildes auf eine endliche Sensorflächen gegliedert werden [34]. Die Punkte des Gitters seien also gegeben durch

$$
\mathbf{x}_{m, n}=[m \Delta x, n \Delta y], \quad m, n \in \mathbb{Z} .
$$

Die Abtastung kann beschrieben werden durch

$$
\begin{aligned}
& g_{s}(\mathbf{x})=g(\mathbf{x}) \sum_{m, n} \delta\left(\mathbf{x}-\mathbf{x}_{m, n}\right) \\
& \uparrow \mathcal{F} \\
& \hat{g}_{s}(\mathbf{k})=\sum_{m, n} \hat{g}_{s}\left(\mathbf{k}-\mathbf{k}_{u, v}\right) \text {, }
\end{aligned}
$$

wobei mit

$$
\mathbf{k}_{u, v}=\left(\begin{array}{c}
u \Delta k_{x} \\
v \Delta k_{y}
\end{array}\right)=\left(\begin{array}{c}
2 \pi u / \Delta x \\
2 \pi v / \Delta y
\end{array}\right)
$$


die Punkte des reziproken Gitters gegeben sind. Damit kann das Abstasttheorem wie folgt formuliert werden:

Ist das Spektrum $\hat{g}(\mathbf{k})$ einer kontinuierliche Funktion $g(\mathbf{x})$ bandbegrenzt, d.h.

$$
\hat{g}(\mathbf{k})=0, \quad \forall\left|k_{i}\right| \geq \Delta k_{i} / 2,
$$

dann kann es aus mit Schrittweiten

$$
\Delta x_{i}=2 \pi / \Delta k_{i}
$$

abgetasteten Punkten exakt rekonstruiert werden.

Bisher wurde noch keine Einschränkung bezgl. der Größe des Gitters, d.h. der Größe des abgetasteten Bildes, gemacht. Diese Begrenzung wird nun durch eine allgemeine Fensterfunktion $w(x)$ beschrieben:

$$
\begin{aligned}
g_{l}(\mathbf{x})= & g_{s}(\mathbf{x}) \cdot w(\mathbf{x}) \\
& \uparrow \mathcal{F} \\
\hat{g}_{l}(\mathbf{k})= & \hat{g}_{s}(\mathbf{k}) * \hat{w}(\mathbf{k}),
\end{aligned}
$$

d.h. das Spektrums des abgetasteten Bildes wird im Fourierraum mit der Fouriertransformierten der Fensterfunktion gefaltet. Die Abtastung führt zu einer Begrenzung der Wellenzahl. Die Begrenzung der Bildgröße hingegen bestimmt die Auflösung der Wellenzahl.

Quantisierung Nach der Digitalisierung haben die Pixel immer noch kontinuierliche Grauwerte, die durch die Quantisierung auf eine begrenzte Zahl $Q$ diskreter Grauwerte abgebildet werden:

$$
\left[0, \infty\left[\longmapsto\left\{g_{0}, g_{1}, \ldots, g_{Q-1}\right\}=G .\right.\right.
$$

Die Quantisierung führt zu Fehlern, die im folgenden diskutiert werden sollen. Haben alle Quantisierungsstufen gleiche Abstände $\Delta g$ und sind sie alle gleich wahrscheinlich, so ergibt sich die Varianz aufgrund der Quantisierung durch [34]

$$
\sigma_{q}^{2}=\frac{1}{\Delta g} \int_{g_{q}-\Delta g / 2}^{g_{q}+\Delta g / 2}\left(g-g_{q}\right)^{2} \mathrm{~d} g=\frac{1}{12}(\Delta g)^{2} .
$$

Die Quantisierungsstufen sind i.a. durch Nichtlinearitäten der Analog-Digital-Wandler nicht exakt äquidistant.

Als Beispiel für Messungen mit Subpixelgenauigkeit wird die Bestimmung der Position einer Kante in einem eindimensionalen Bild diskutiert. In Abb. 2.7 (a) wird die Kante ohne vorherige Glättung abgetastet ( $\delta$-Abtastung). Die Position der Kante kann nicht genauer bestimmt werden als die Breite eines Pixels. Ist die Kante hingegen geglättet (Abb. 2.7 (b), (c)), so ist eine genauere Bestimmung ihrer Position möglich. Erstaunlicherweise erlaubt daher ein unscharfes Bild zunächst eine genauere Positionsbestimmung als ein scharfes Bild [33]. Nimmt die Unschärfe weiter zu, so nimmt die Genauigkeit der Positionsbestimmung jedoch wieder ab. Es gibt daher ein Optimum der Genauigkeit, mit der Positionen in einem Bild gemessen werden können. Ein typischer, im Experiment erreichbarer Wert liegt bei 0.1 bis 0.01 Pixel [53].

\subsubsection{Modellierung}




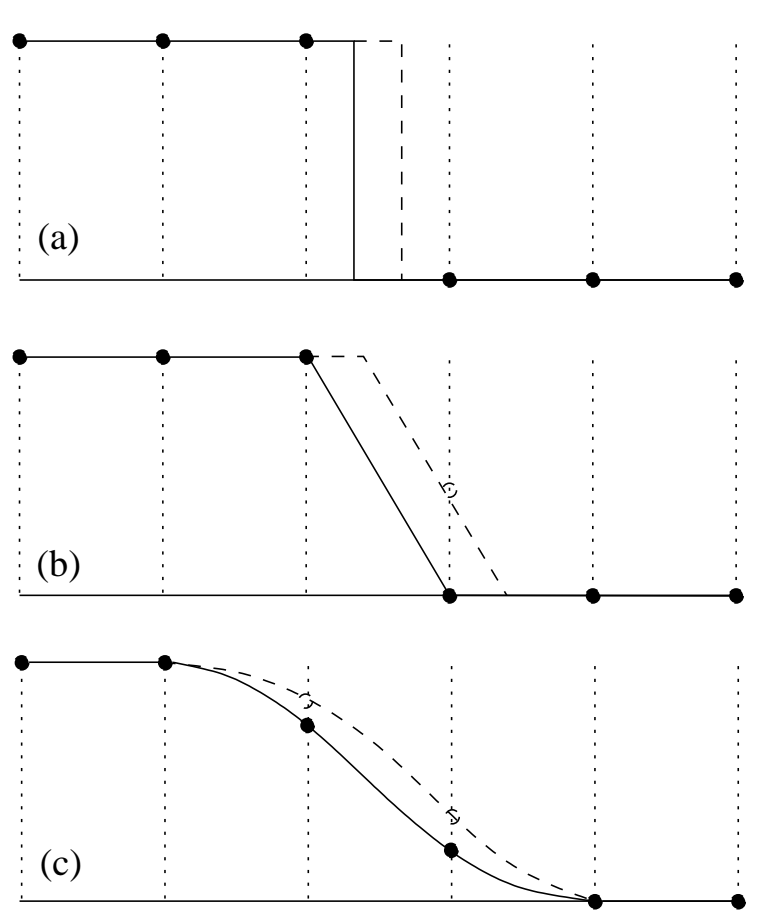

Abbildung 2.7.: Illustratives Beispiel für die subpixelgenaue Bestimmung der Position einer Kante [33]. (a) $\delta$-Abtastung ohne vorherige Glättung; (b) Standard-Abtastung; (c) Gauss-Abtastung.

Kameramodell Grundlage für die Entwicklung eines photogrammetrischen oder stereoskopischen Meßverfahrens ist die Modellierung und Kalibrierung der Geometrie des optischen Aufbaus und der Bildaufnahme. In diesem Abschnitt wird ein Kameramodell beschrieben, das die optische Abbildung durch eine Lochkamera in idealisierter Form beschreibt. Das reale System unterscheidet sich hiervon durch Fehler durch optische Verzeichnungen und elektronische Störungen. Diese Fehler werden in dem Modell berücksichtigt.

Ein Kameramodell $\mathcal{C}^{\Phi}$ beschreibt formal die Abbildung

$$
\begin{aligned}
\mathcal{C}^{\Phi}: \mathbb{R}^{3} & \longmapsto \mathbb{R}^{2} \\
\mathbf{x}_{w} & \longrightarrow \mathbf{x}_{f}
\end{aligned}
$$

eines dreidimensionalen Objektpunktes $\mathbf{x}_{w}=\left(x_{w}, y_{w}, z_{w}\right)^{t}$ auf die zweidimensionalen Bildschirmspeicherkoordinaten $\mathbf{x}_{f}=\left(x_{f}, y_{f}\right)^{t}$.

Die Koordinatentransformation vom dreidimensionalen Objekt- bzw. Weltkoordinatensystem $\left(x_{w}, y_{w}, z_{w}\right)$ auf das ebenfalls dreidimensionale Kamerakoordinatensystem $(x, y, z)$ wird beschrieben durch

$$
\left(\begin{array}{l}
x \\
y \\
z
\end{array}\right)=\underline{\underline{\mathbf{R}}}\left(\begin{array}{c}
x_{w}-x_{0} \\
y_{w}-y_{0} \\
z_{w}-z_{0}
\end{array}\right),
$$

wobei die orthogonale $3 \times 3$ Rotationsmatrix $\underline{\underline{\mathbf{R}}}$

$$
\underline{\underline{\mathbf{R}}}=\left(\begin{array}{lll}
r_{11} & r_{12} & r_{13} \\
r_{21} & r_{22} & r_{23} \\
r_{31} & r_{32} & r_{33}
\end{array}\right)
$$


(a)

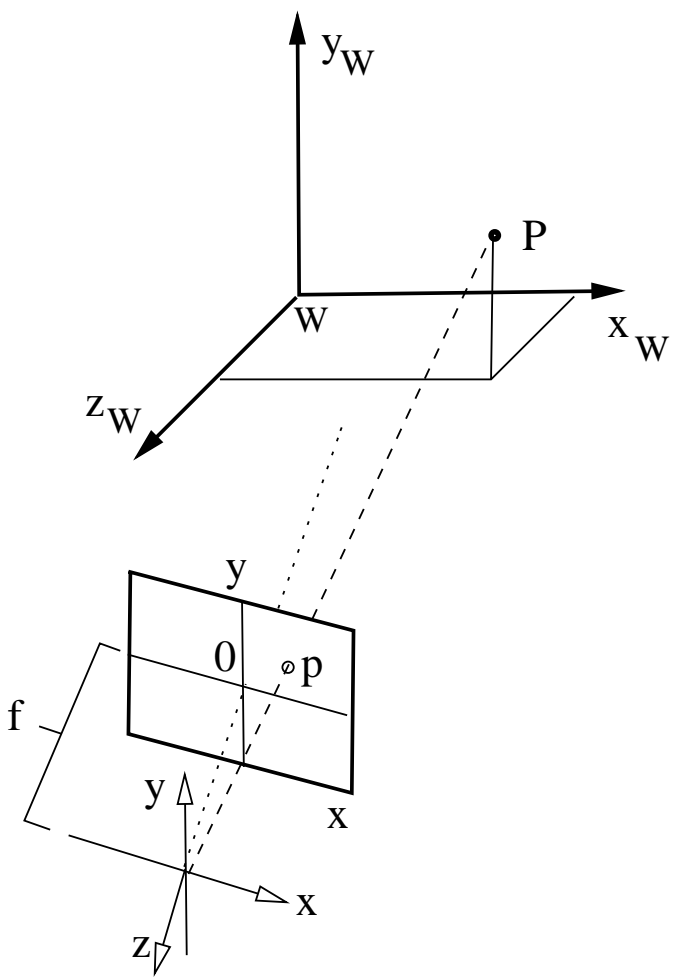

(b)

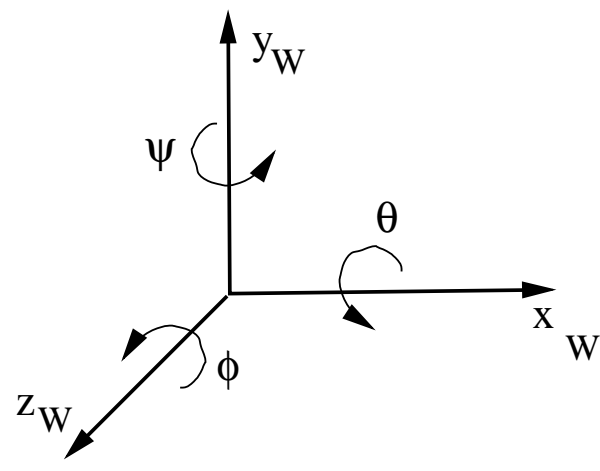

Abbildung 2.8.: (a) Welt- und Kamera-Koordinaten-System. (b) Drehwinkel im WeltKoordinaten-System.

durch die Drehwinkel $\phi, \theta$ und $\psi$ parametrisiert ist. Die Transformation von dem dreidimensionalen Kamerakoordinatensystem $(x, y, z)$ auf die idealen, d.h. unverzerrten Bildkoordinaten $x_{u}, y_{u}$ wird durch die Zentralprojektion

$$
\begin{aligned}
& x_{u}=x_{h}-f \frac{x}{z}, \\
& y_{u}=y_{h}-f \frac{y}{z}
\end{aligned}
$$

bzw. explizit durch

$$
\begin{aligned}
& x_{u}=x_{h}-f \frac{r_{11}\left(x_{w}-x_{0}\right)+r_{12}\left(y_{w}-y_{0}\right)+r_{13}\left(z_{w}-z_{0}\right)}{r_{13}\left(x_{w}-x_{0}\right)+r_{23}\left(y_{w}-y_{0}\right)+r_{33}\left(z_{w}-z_{0}\right)}, \\
& y_{u}=y_{h}-f \frac{r_{12}\left(x_{w}-x_{0}\right)+r_{22}\left(y_{w}-y_{0}\right)+r_{23}\left(z_{w}-z_{0}\right)}{r_{13}\left(x_{w}-x_{0}\right)+r_{23}\left(y_{w}-y_{0}\right)+r_{33}\left(z_{w}-z_{0}\right)}
\end{aligned}
$$

mit der Fokallänge $f$ und den Komponenten des Prinzipalpunktes $x_{h}$ und $y_{h}$ beschrieben. Die Gln. (2.15) werden in der Photogrammetrie als Kollinearitätsgleichungen bezeichnet.

Die Gln. (2.15) beschreiben eine ideale Abbildung. Linsenfehler und Fehljustierung optischer Komponenten führen jedoch zu Abbildungsfehlern. Durch eine Modellierung dieser Verzeichnungen ist eine numerische Korrektur im Rahmen der Kalibrierung möglich. Wir beschränken uns hier auf die radialen und tangentialen Verzeichungen. Die radialen Verzeichnungen werden beschrieben durch den Ansatz

$$
\begin{aligned}
& x_{d}=x_{u}+\delta_{x}^{r}+\delta_{x}^{t}, \\
& y_{d}=y_{u}+\delta_{y}^{r}+\delta_{y}^{t}
\end{aligned}
$$


(a)
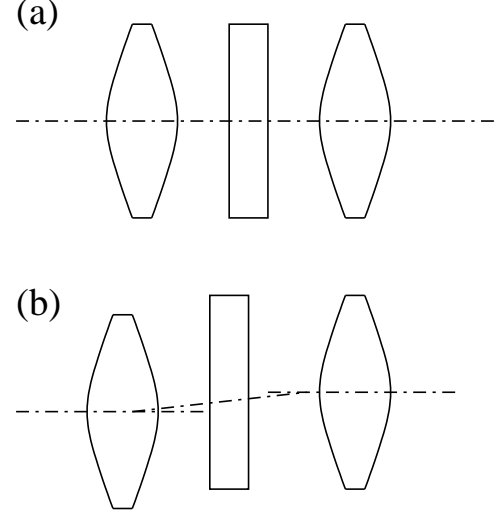

Abbildung 2.9.: Schematische Darstellung eines tangentialen Linsenfehlers. (a) Ideale Anordnung der optischen Komponenten auf der optischen Achse. (b) Nichtzentrierte Anordnung.

mit

$$
\left(\begin{array}{l}
\delta_{x}^{r} \\
\delta_{y}^{r}
\end{array}\right)=\left(\begin{array}{l}
x_{u} \\
y_{u}
\end{array}\right)\left(\kappa_{1} r^{2}+\kappa_{2} r^{4}+\kappa_{3} r^{6}+\ldots\right),
$$

wobei $\kappa_{i}$ die radialen Verzeichnungskoeffizienten bezeichnen und $r^{2}=x_{u}^{2}+y_{u}^{2}$ gilt. Tangentiale Verzeichnungen entstehen, wenn die Symmetrieachsen der optischen Komponenten von der optischen Achse abweichen (Abb. 2.9). Diese Verzeichnungen können beschrieben werden durch

$$
\begin{aligned}
& \delta_{x}^{t}=p_{1}\left(r^{2}+2 x_{u}^{2}\right)+2 p_{2} x_{u} y_{u} \\
& \delta_{y}^{t}=2 p_{1} x_{u} y_{u}+p_{2}\left(r^{2}+2 y_{u}^{2}\right),
\end{aligned}
$$

mit den Koeffizienten $p_{1}$ und $p_{2}$ der tangentialen Verzeichnung. Die verzeichneten Koordinaten $\left(x_{d}, y_{d}\right)$ werden durch die Bildwandlerkarte (Framegrabber) auf die Bildspeicherkoordinaten $\left(x_{f}, y_{f}\right)$ durch

$$
\begin{aligned}
& x_{f}=\frac{x_{d}}{d_{x}}, \\
& y_{f}=\frac{y_{d}}{d_{y}}
\end{aligned}
$$

abgebildet, wobei $d_{x}$ und $d_{y}$ den Abstand der Sensorelemente in $x$-Richtung (Zeile) bzw. $y$ Richtung (Spalte) bezeichnen. In der Gl. (2.19) können Abbildungsfehler durch Synchronisationsprobleme mit Hilfe einer affinen zweiparametrigen Abbildung berücksichtigt werden.

Die Gln. (2.12)-(2.19) sind ein 13 parametriges Kameramodell mit dem Parametervektor

$$
\Phi=\left(x_{0}, y_{0}, z_{0}, \phi, \theta, \psi, \kappa_{1}, \kappa_{2}, \kappa_{3}, p_{1}, p_{2}, s_{1}, s_{2}\right),
$$

welcher die jeweils drei Parameter der äußeren und inneren Orientierung, sowie die fünf Parameter der Linsenverzeichung und zwei Parameter der elektronischen Korrektur $\left(s_{1}, s_{2}\right)$ zusammenfaßt. Diese Parameter können durch eine nichtlineare Regression für einen Satz bekannter Paßpunkte eines Kalibrierobjektes bestimmt werden. Daher ist die Genauigkeit der Bestimmung von $\mathrm{Pa} ß$ - oder Kalibrierpunkten für die Genauigkeit des Meßverfahrens von besonderer Bedeutung. 


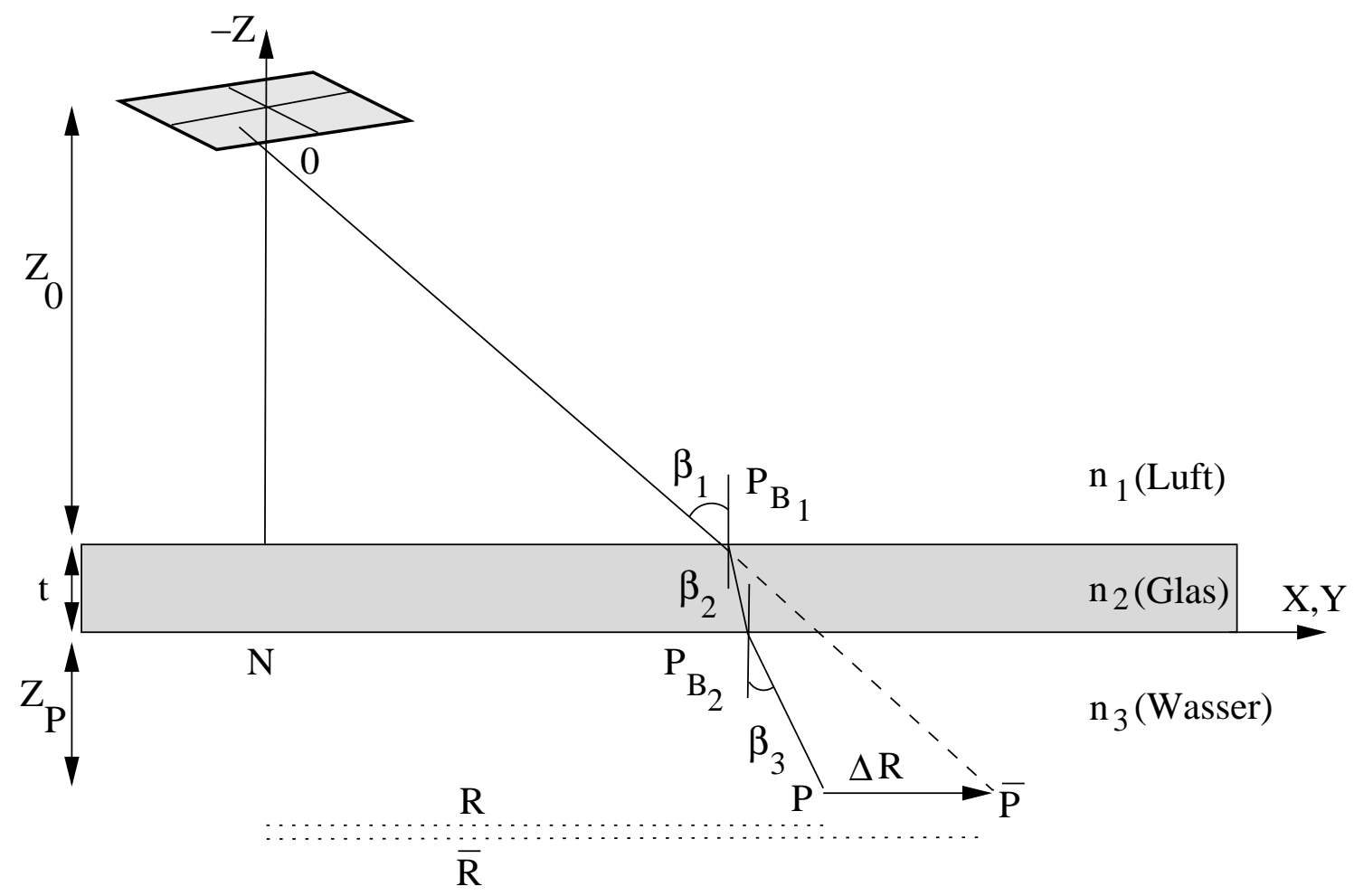

Abbildung 2.10.: Geometrie der Phasengrenze.

Phasengrenzen Es wird nun der Einfluß einer ebenen Grenzfläche mit der konstanten Schichtdicke $t$ auf die Abbildung eines Objektpunktes betrachtet. Die Lage der Grenzfläche sei im 3D Koordinatensystem $(x, y, z)$ definiert durch den Aufpunkt $\mathbf{p}$ und den Normaleneinheitsvektor $\mathbf{n}$ (Abb. 2.10). O.B.d.A. wird der Aufpunkt durch den Schnittpunkt der Grenzfläche mit der optischen Achse, d.h. der $z$-Achse, bestimmt. Für die Brechungsindizes $n_{i}$ gilt das Brechungsgesetz

$$
n_{1} \sin \beta_{1}=n_{2} \sin \beta_{2}=n_{3} \sin \beta_{3} .
$$

Aus der Geometrie in Abb. 2.10 folgt dann

$$
\begin{aligned}
R & =Z_{0} \tan \beta_{1}+t \tan \beta_{2}+Z_{p} \tan \beta_{3}, \\
\bar{R} & =\left(Z_{0}+t+Z_{p}\right) \tan \beta_{1},
\end{aligned}
$$

woraus die Verschiebung des Objektpunktes $P$ auf $\bar{P}$ bestimmt werden kann.

Kalibrierung des Kameramodells Die Kalibrierung des Kameramodell ist eine notwendige Voraussetzung für die Verwendung des bildgebenden Verfahrens zu Meßzwecken. Um das beschriebene Kameramodell zu kalibrieren, muß ein Kalibrierobjekt mit genau vermessenen Kalibrier- oder Paßpunkten aufgezeichnet werden. Die erreichbare Genauigkeit einer Messung wird durch die Genauigkeit bestimmt, mit der die Kalibrierpunkte bekannt sind. Die Praxis zeigt, daß diese Genauigkeit etwa eine Größenordnung besser sein sollte als die beabsichtigte Meßgenauigkeit. Daher ist bei der Anfertigung und Vermessung der Kalibrierobjekte große Sorgfalt notwendig. Die Kalibrierobjekte können zwei- oder dreidimensional sein. In dieser Arbeit wurden gute Ergebnisse mit ebenen Kalibrierobjekten erzielt, die durch photographische Verkleinerung einer Vorlage auf ein Diapositiv abgebildet werden. Die Lage der Kalibrierpunkte 
(a)

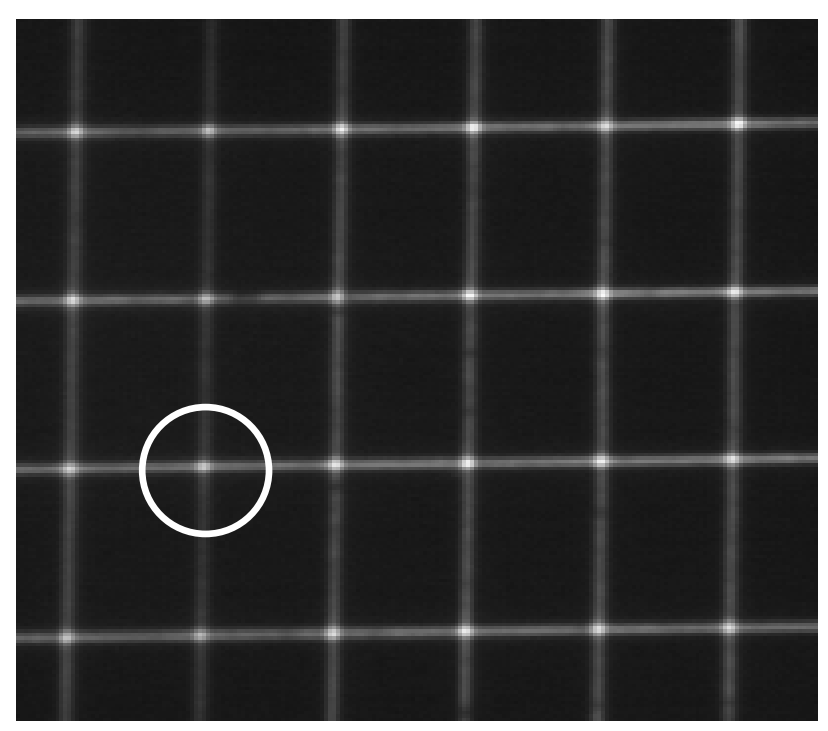

(b)

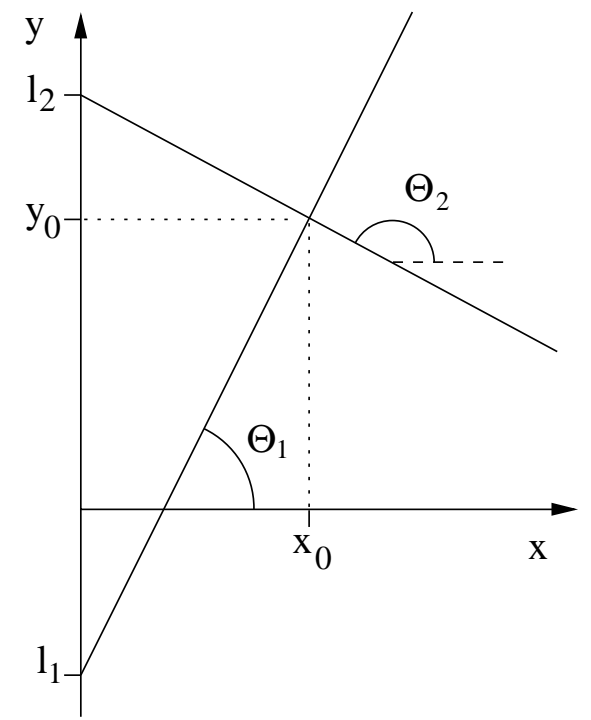

Abbildung 2.11:: (a) Ausschnitt einer Aufnahme eines ebenen Kalibrierobjektes, in der die Umgebung eines Kalibrierpunktes markiert ist. (b) Geometrie der Modellfunktion. Die freien Parameter Gl. (2.24) werden mit Hilfe einer nichtlinearen Regression ermittelt. Aus Gl. (2.26) werden dann die Koordinaten $\left(x_{0}, y_{0}\right)$ des Schnittpunktes bestimmt.

muß dann unter einem Meßmikroskop ermittelt werden. Die verwendeten Kalibrierobjekte haben typischerweise 100 bis 400 Kalibrierpunkte, die gleichmäßig über die Bildebene angeordnet werden. Ein Beispiel eines Ausschnittes eines solchen Kalibrierobjektes ist in Abb. 2.12 dargestellt. Die Schätzung der Position der Kalibrierpunkte in der Aufnahme wird durch eine einfache Mustererkennung gewonnen. Eine subpixelgenaue Bestimmung der Position kann dann durch die Anpassung einer Modellfunktion einer Grauwertverteilung

$$
\begin{array}{r}
f(x, y)=a+b x+c y+d_{1}^{2} \exp \left(-e_{1}^{2}\left(x \cos \theta_{1}+y \sin \theta_{1}+l_{1}\right)^{2}\right) \\
+d_{2}^{2} \exp \left(-e_{2}^{2}\left(x \cos \theta_{2}+y \sin \theta_{2}+l_{2}\right)^{2}\right)
\end{array}
$$

durchgeführt werden. Die Koeffizienten $a, b$ und $c$ beschreiben die konstanten bzw. die linearen Komponenten des Grauwerthintergrundes und die Parameter $d_{i}$ und $e_{i}$ die Amplitude und die Breite der Diagonalen. Die geometrische Ausrichtung bezogen auf den Ursprung des Koordinatensystems ist durch die Winkel $\theta_{i}$ und die Achsenabschnitte $l_{i}$ gegeben (Abb. 2.12 (b)). Bezeichnet $g(x, y)$ die gemessene Grauwertverteilung in der Umgebung eines Kalibrierpunktes, so wird dessen Lage bestimmt durch den Parametervektor

$$
p=\left(a, b, c, d_{1}, e_{1}, \theta_{1}, l_{1}, l_{2}, d_{2}, e_{2}, \theta_{2}, l_{2}\right),
$$

für den

$$
\sum_{i, j}|g(x, y)-f(x, y)|
$$

minimal wird. Zur numerischen Berechnung wird die Methode der kleinsten Quadrate nach Levenberg-Marquardt verwendet. Die Koordinaten $\left(x_{0}, y_{0}\right)$ des Kalibrierpunktes sind dann gegeben durch die Lösung des Gleichungssystems

$$
\begin{aligned}
& x_{0} \cos \theta_{1}+y_{0} \sin \theta_{1}+l_{1}=0, \\
& x_{0} \cos \theta_{2}+y_{0} \sin \theta_{2}+l_{2}=0 .
\end{aligned}
$$


(a)

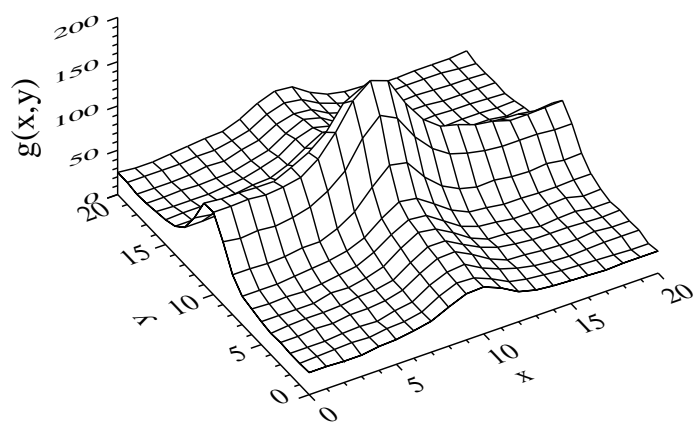

(b)

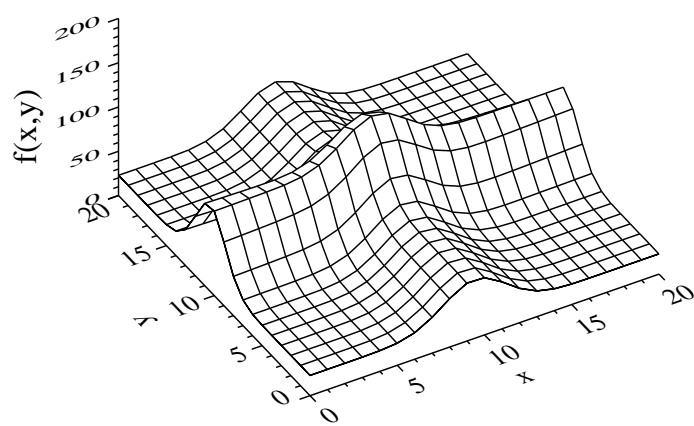

Abbildung 2.12.: Subpixelgenaue Bestimmung der Position der Kalibrierpunkte. (a) Ausschnitt der Grauwertverteilung der Kalibrieraufnahme aus Abb. 2.11. (b) Anpassung der Modellfunktion an die Grauwertverteilung.

Ein Beispiel für eine solche Bestimmung der Kalibrierpunkte ist in Abb. 2.12 dargestellt.

\subsection{Bearbeitung der Bildsequenzen}

In diesem Abschnitt werden die in dieser Arbeit zur Bearbeitung der Bildsequenzen verwendeten Methoden vorgestellt. Die Bildvorbearbeitung umfaßt die elementaren Methoden zur Verbesserung der Qualität der digitalen Bilder. In der darauf folgenden Bildbearbeitung werden die Grundlagen gelegt für die Erkennung von Objekten in dem digitalen Bild. Die einzelnen Bearbeitungsschritte werden jeweils an einem Beispiel illustriert.

\subsubsection{Bildvorbearbeitung}

$\mathrm{Zu}$ den Methoden der elementaren Bildvorbearbeitung zählen die pixelorientierten Verfahren der Kontrastverstärkung und Filter zur Unterdrückung des Meßrauschens und inhomogener Beleuchtung.

Geometrische Korrektur Die Kalibrierung des Kameramodells erlaubt die geometrische Korrektur der durch Abbildungsfehler verursachten Verzeichnungen, die die gemessene Grauwertverteilung des digitalen Bildes von radialen und tangentialen Linsenverzeichnungen befreit.

Kontrastverstärkung Infolge schwieriger Beleuchtungsbedingungen weisen die Aufnahmen oft eine geringe Grauwertdynamik auf. Der visuelle Eindruck des Bildes kann durch Kontrastverstärkung verbessert werden. Allerdings ändert diese Operation nicht die Bildqualität selbst, da die Grauwertauflösung unverändert bleibt. Ein Grauwerthistogramm des Bildes zeigt, ob die Objekte auch bei inhomogener Beleuchtung zuverlässig vom Hintergrund getrennt werden können. Um die Bildqualität zu erhöhen, wurde daher eine Optimierung der Beleuchtung durchgeführt. Eine weitere Möglichkeit der Verbesserung der Bildqualität besteht darin, durch 
Erhöhung der Verstärkung der Bildwandlerkarte oder der Kamerasteuerung die Helligkeit und Auflösung des Bildes zu verbessern; allerdings geht dies auf Kosten eines erhöhten Rauschpegels (Abb. 2.13 (a), (b)). Ein Beispiel für die Kontrastverstärkung ist in Abb. 2.13 (b) gezeigt.

Filterung Das kontrastverstärkte digitale Bild in Abb. 2.13 (b) zeigt eine deutlich inhomogene Hintergrundintensität und einen relativ hohen Rauschpegel. Mit einem Bandpassfilter kann das Rauschen reduziert und der inhomogene Hintergrund vom Bild subtrahiert werden. Als Bandpassfilter wird ein Wavelet-Filter eingesetzt. Das gefilterte Bild ist in Abb. 2.13 (c) dargestellt.

\subsubsection{Bildbearbeitung}

Grundlegend für die Analyse der digitalen Bildern ist das Erkennen von Objekten. Dies wird von den im Folgenden beschriebenen Bildbearbeitungsverfahren geleistet. Das Erkennen von Objekten ist die Fähigkeit, Objekte von ihrem Hintergrund zu trennen. Die Verfahren zur Bildbearbeitung lassen sich in zwei Klassen einteilen:

- Objekt-bezogenene Verfahren, die muster- oder modellbasiert sind. Objekte werden in einem digitalen Bild anhand vorgegebener Muster bzw. Eigenschaften erkannt. Das Segmentierungsmerkmal ist ein Schwellwert der Grauwertverteilung.

- Grauwertorientierte Verfahren. Im Gegensatz zu den Objekt-bezogenen Verfahren betrachten diese struktur-basierten Verfahren die Grauwertverteilung eines digitalen Bildes, ohne a priori Annahmen über die Gestalt der Objekte zu machen. Das Segmentierungsmerkmal ist das Maß für die lokale Orientierung oder Struktur der Grauwerte, wie sie der Struktur-Tensor liefert.

Objektbezogende Segmentierung durch Schwellwert Die pixelorientierte Segmentierung ist die einfachste Möglickeit der Objekterkennung. Als Segmentierungsmerkmal wird hier einem Pixel abhängig von seinem Grauwert eine Eigenschaft zugeordnet, d.h. ob er zu einem Objekt gehört oder zum Hintergrund. Im Gegensatz zu diesen pixelorientierten Segmentierungsverfahren basieren regionenorientierte Verfahren auf der Eigenschaft, daß Objekte zusammenhängende Pixel haben. Die pixelorientierte Segmentierung ist dann besonders erfolgreich, wenn das Histogramm der Grauwerte des Bildes eine bimodale Verteilung zeigt. Dies ermöglicht eine zuverlässige Separierung des Objektes vom Bildhintergrund. Wenn der Hintergrund keine homogene Beleuchtung hat, so kann für die Segmenterung ein adaptiver Schwellwert verwendet werden. Die Wahl des Schwellwertes hängt von den Eigenschaften jeder einzelnen Bildsequenz ab; i.d.R. liefert ein Schwellwert, der etwa 20\% über dem mittleren lokalen Grauwerthintergrund liegt, gute Ergebnisse. Bei im Bild dicht nebeneinander liegenden Objekten kann ein zu niedrig gewählter Schwellwert dazu führen, daß die Objekte nicht mehr getrennt, sondern als ein einziges, nahezu elliptisches Objekt registriert werden. Ein Beispiel für eine Segmentierung ist in Abb. 2.13 (d) dargestellt.

Neben dem Grauwert kann auch die lokale Orientierung der Pixel als Segmentierungskriterium verwendet werden. Diese Methode beruht auf der Annahme, daß die Objekte in dem Bild durch eine bestimmte lokale Grauwertstruktur charakterisiert sind. Die Segmentierung mit Hilfe des Bestimmtheitsmaßes der Grauwerte wurde insbesondere für die Segmentierung von Streulichtaufnahmen verwendet. Das Verfahren erlaubt eine robuste Objekterkennung, die unabhängig ist von der Amplitude der Grauwerte. Objekte können auch dann erkannt werden, wenn sie sich nicht vom mittleren Grauwert des Hintergrundes unterscheiden. 
Grauwertbezogene Segmentierung durch Struktur-Tensor Bisher wurden punktbzw. pixelorientierte Verfahren dargestellt. Im Folgenden werden lokale Umgebungen betrachtet, die durch eine Orientierung beschrieben werden können. Dies ist eine notwendige Voraussetzung für die Analyse komplexer Strukturen. Der Struktur-Tensor ist ein Verfahren, das die lokale Struktur einer Grauwertverteilung charakterisiert. Der Vorteil dieser Methode ist, daß sie effizient als Filter-Operator implementiert werden kann. Die Struktur-Tensor-Methode im Ortsraum ist das Äquivalent zu der Trägheitstensor-Methode im Fourier-Raum. Ausgangspunkt ist die Fragestellung, wie lokale Struktur im Ortsraum charakterisiert werden kann. Ausgehend von den Forderungen nach Eindeutigkeit, Uniformität und polarer Separabilität, folgerte Knutsson [39], daß die lokale Struktur in einem $n$-dimensionalen Raum durch einen symmetrischen $n \times n$-Tensor der Form

$$
\underline{\underline{\mathbf{J}}}=|\mathbf{x}|^{-1} \mathbf{x x}^{T} \quad \text { mit } \quad \mathbf{x} \in R^{n}
$$

dargestellt werden kann. In dieser Darstellung ist die Orientierung invariant bzgl. Rotationen um $\pi$. Eine Repräsentation der lokalen Struktur einer Grauwertverteilung $g(\mathbf{x})$ ist gegeben durch den Struktur-Tensor

$$
\underline{\underline{\mathbf{J}}}(\mathbf{x})=\int_{-\infty}^{\infty} \mathrm{d}^{n} x^{\prime} w\left(\mathbf{x}-\mathbf{x}^{\prime}\right) \nabla g\left(\mathbf{x}^{\prime}\right) \nabla^{T} g\left(\mathbf{x}^{\prime}\right)
$$

bzw. komponentenweise

$$
J_{p q}=\int_{-\infty}^{\infty} \mathrm{d}^{n} \mathbf{x}^{\prime} w\left(\mathbf{x}-\mathbf{x}^{\prime}\right)\left(\frac{\partial g\left(\mathbf{x}^{\prime}\right)}{\partial \mathbf{x}_{p}^{\prime}} \frac{\partial g\left(\mathbf{x}^{\prime}\right)}{\partial \mathbf{x}_{q}^{\prime}}\right)
$$

wobei $w\left(\mathbf{x}-\mathbf{x}^{\prime}\right)$ eine geeignet zu wählende Fensterfunktion ist. Die lokale Struktur der Grauwertverteilung eines Bildes kann mit dem Struktur-Tensor-Operator

$$
\mathcal{J}_{p q}=\mathcal{B}\left(\mathcal{D}_{p} \cdot \mathcal{D}_{q}\right)
$$

bestimmt werden. $\mathcal{D}_{p}$ bzw. $\mathcal{D}_{q}$ bezeichnen Differentiationsoperatoren und $\mathcal{B}$ einen Glättungsoperator. Details zu der effizienten numerischen Implementation der Operatoren als rekursives Glättungfilter und Ableitungsfilter nach dem B-Spline-Verfahren sind in [34] dargestellt. Die pixelweise Multiplikation wird mit - bezeichnet. Das lokale Kohärenzmaß $C$ der Grauwertverteilung wird dann mit Gl. (2.30) durch

$$
C=\frac{\left(\mathcal{J}_{11}-\mathcal{J}_{22}\right) \cdot\left(\mathcal{J}_{11}-\mathcal{J}_{22}\right)+4 \mathcal{J}_{12} \cdot \mathcal{J}_{12}}{\left(\mathcal{J}_{11}+\mathcal{J}_{22}\right) \cdot\left(\mathcal{J}_{11}+\mathcal{J}_{22}\right)}=\left(\frac{\mathcal{J}_{1}-\mathcal{J}_{2}}{\mathcal{J}_{1}+\mathcal{J}_{2}}\right)^{2} \quad \text { mit } \quad 0 \leq C \leq 1
$$

definiert. Für eine isotrope Grauwertverteilung ist das Kohärenzmaß $C=0\left(\mathcal{J}_{1}=\mathcal{J}_{2}>0\right)$ und für eine ideal ausgerichtete Struktur gilt $C=1\left(\mathcal{J}_{1}=0, \mathcal{J}_{2}>0\right)$. Damit kann das lokale Kohärenzmaß als Segmentierungsmerkmal verwendet werden. Für den Orientierungswinkel $\Phi$ der Grauwertstruktur gilt

$$
\tan 2 \Phi=\frac{2 \mathcal{J}_{12}}{\mathcal{J}_{11}-\mathcal{J}_{22}} .
$$

Ein Schwellwert für das lokale Kohärenzmaß $C$ führt zu einer Segmentierung des Bildes und damit zu einem binären Bild.

Die binären Objekte in segmentierten Bildern können in geeigneten Datenstrukturen, d.h. in Quadtrees, Lauflängen- oder Kettencode, kompakt gespeichert werden. Der in dieser Arbeit verwendete Lauflängencode erlaubt eine effiziente Implementierung der BildverarbeitungsAlgorithmen. 


\subsection{Analyse der Bildsequenzen}

Mit der Analyse der Bildsequenzen wird der Übergang von der Bearbeitung digitaler Bilder zu Merkmalsbildern vollzogen. Merkmalsbilder entstehen durch die Extraktion von Strukturen und die Beschreibung ihrer Eigenschaften. In den uns interessierenden Aufnahmen akustischer Kavitationsblasenfelder ist dies die geometrische Charakterisierung der Blasen. Auf der Grundlage dieser Eigenschaften ist eine Klassifikation der Blasen möglich. Für die physikalische Interpretation der Bildsequenzen steht die Analyse der Bewegung im Vordergund. Auch die Bewegung eines Objektes ist eine in das Merkmalsbild eingehende Eigenschaft.

\subsubsection{Klassifikation}

Als Klassifikation bezeichnet man das Einteilen von Objekten nach den ihnen zugewiesenen Eigenschaften oder Merkmalen.

Die Segmentierung liefert ein binäres Bild: Ein Bildpunkt hat den Wert eins, wenn er zu einem Objekt gehöhrt und den Wert null, wenn er zu dem Hintergrund gehört. In diesem binären Bild müssen nun die Pixel einzelnen Objekten zugeordnet werden. Ein geeignetes Verfahren hierfür ist ein Cluster-Algorithmus. Sind die Pixel der Objekte einzelnen Objekten zugeordnet, so können ihre Objekteigenschaften bestimmt werden.

Eine einfache Beschreibung der Eigenschaften eines Objektes ist durch seine geometrische Form möglich. Zu diesen Formparametern gehören neben anderen v.a. Schwerpunkt, Fläche, Orientierung und Exzentrizität. Es kann gezeigt werden, daß die Momente einer Verteilung eine vollständige, invariante und robuste Formbeschreibung eines Objektes erlauben [67]. Eine Formbeschreibung ist invariant, wenn die durch sie bestimmte Eigenschaft bei Translation, Rotation, Skalierung und Kontrast- oder Helligkeitsveränderung des Objektes erhalten bleibt. Die Momente der Grauwertfunktion $g(\mathbf{x})$ eines Objektes werden definiert durch

$$
m_{p, q}=\int(x-\langle x\rangle)^{p}(y-\langle y\rangle)^{q} g(\mathbf{x}) \mathrm{d} \mathbf{x} \quad \text { und } \quad p, q=0,1,2, \ldots
$$

und

$$
\langle x\rangle=m_{1,0} / m_{0,0} \quad\langle y\rangle=m_{0,1} / m_{0,0}
$$

wobei die Integration über die Fläche des Objektes gebildet wird. Der Vektor $(\langle x\rangle,\langle y\rangle)$ wird als Schwerpunkt bezeichnet. Da sich alle in Gl. (2.33) definerten Momente auf den Schwerpunkt beziehen, werden sie als zentrale Momente bezeichnet. In binarisierten Bildern reduziert sich Gl. (2.33) auf

$$
m_{p, q}=\sum(x-\langle x\rangle)^{p}(y-\langle y\rangle)^{q} .
$$

Summiert wird über alle Pixel, die zu dem Objekt gehören. Die drei Momente zweiter Ordnung, für die $p+q=2$ ist, bilden die Komponenten des Trägheitstensors ${ }^{1}$

$$
\underline{\underline{\mathbf{J}}}=\left(\begin{array}{cc}
m_{2,0} & -m_{1,1} \\
-m_{1,1} & m_{0,2}
\end{array}\right)
$$

Mit Hilfe dieser Momente können nun die Orientierung und die Exzentrizität des Objektes bestimmt werden. Die Orientierung eines Objektes wird definiert durch den Winkel $\theta$ zwischen

\footnotetext{
${ }^{1}$ Die Grauwertverteilung kann als Flächendichte einer massebelegten Platte und der Tensor Gl. (2.36) als Analogon zum Trägheitstensor in der klassischen Mechanik interpretiert werden (siehe z.B. [44]).
} 
(a)

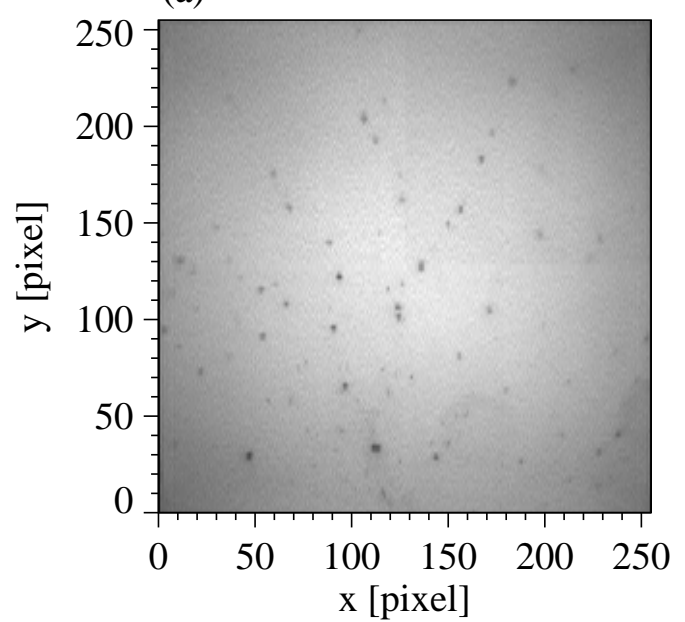

(c)

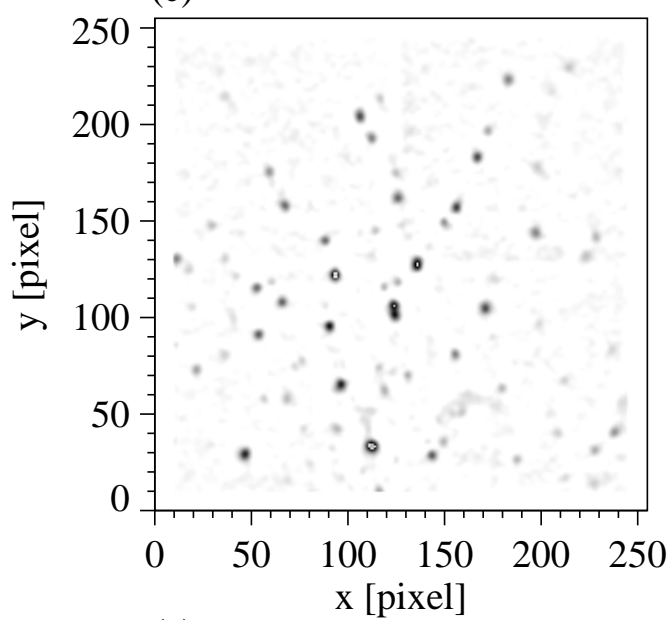

(e)

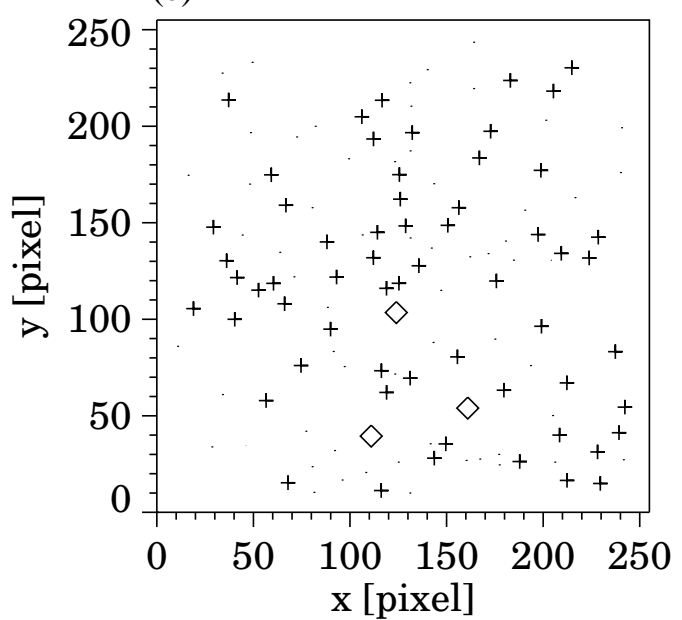

(b)

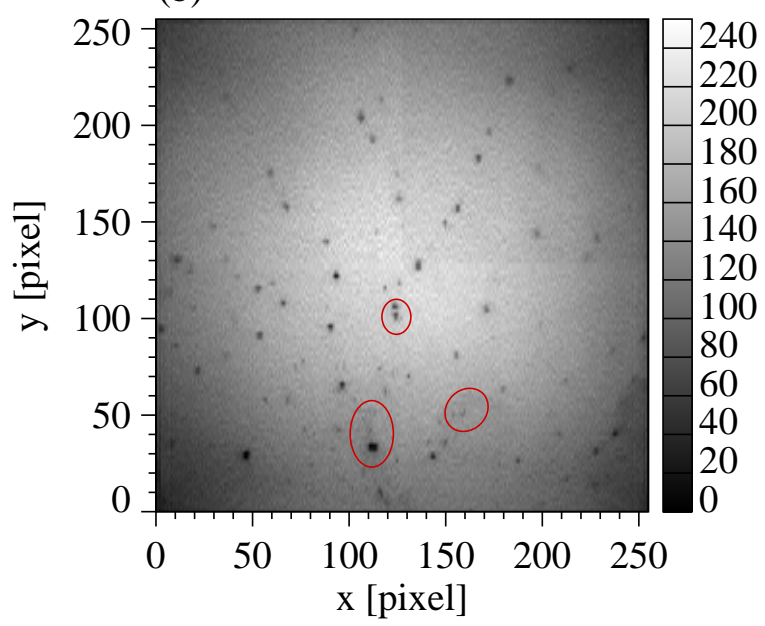

(d)

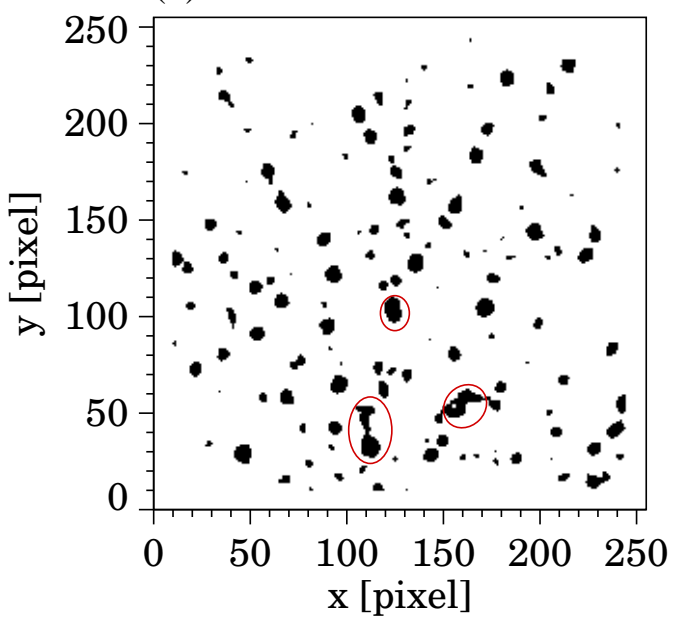

Abbildung 2.13.: Bearbeitung einer Bildsequenz. (a) Originalbild. (b) Kontrastverstärktes Bild. Deutlich erkennbar ist die Inhomogenität der Beleuchtung. (c) Bandpassgefiltertes Bild. (d) Segmentiertes Bild. Markiert sind Objekte, deren Formen oder Flächen auffällig sind. Diese Objekte können im Merkmalsraum identifiziert werden. (e) Objektschwerpunkte. Mit $\diamond$ und $\cdot$ sind auffällige Objekte markiert (siehe Abb. 2.14). 


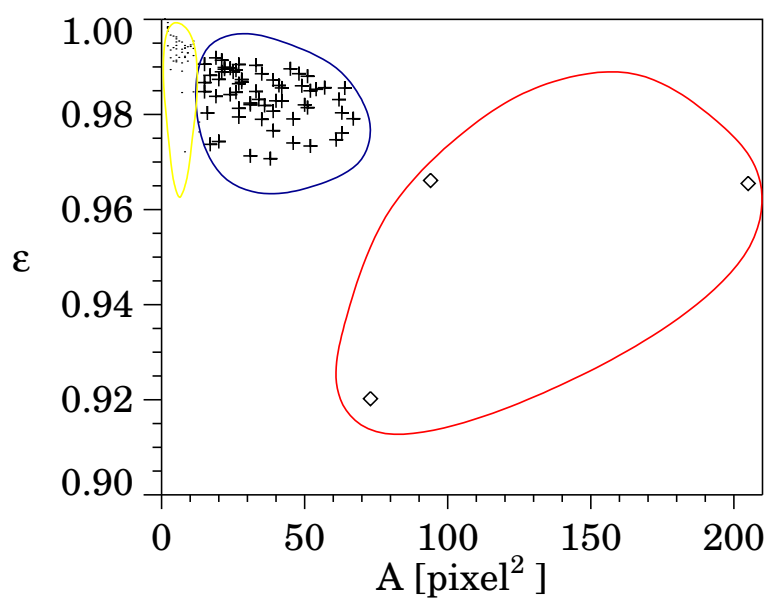

Abbildung 2.14.: Der von der Objektfläche $A$ und ihrer Exzentrizität $\epsilon$ aufgespannte Merkmalsraum für das in Abb. 2.13 gezeigte Beispiel. Rot markiert sind die Objekte, die durch ihre Größe und/oder ihre Exzentrizität auffällig sind und sich hierdurch von den anderen Objekte (blau markiert) deutlich unterscheiden. Eine Klassifizierung der Objekte ist daher durch eine Cluster-Analyse im Merkmalsraum möglich.

der $x$-Achse und der Hauptachse mit dem kleinsten Eigenwert des Tensors Gl. (2.36). In dieser Richtung hat das Objekt seine größte Ausdehnung:

$$
\theta=\frac{1}{2} \arctan \frac{2 m_{1,1}}{m_{2,0}-m_{0,2}} .
$$

Als Maß für die Exzentrizität $\varepsilon$ wird die Größe

$$
\varepsilon=\frac{\left(m_{2,0}^{2}-m_{0,2}^{2}+4 m_{1,1}^{2}\right)}{\left(m_{2,0}+m_{0,2}\right)^{2}} \quad \text { mit } \quad \varepsilon \in[0,1]
$$

definiert. Sie ist null für ein Objekt mit vollständig isotroper Grauwertverteilung und eins für eine Grauwertverteilung mit idealer Orientierung.

Der $p$-dimensionale Merkmalsraum wird durch eine Gruppe von $p$ Eigenschaften aufgespannt. Ein Objekt im digitalen Bild wird repräsentiert durch einen Vektor im Merkmalsraum. Wichtig bei der Auswahl der Merkmale ist, daß sie komplementär zueinander sind und neue Information enthalten. In diesem Merkmalsraum sind Objekte mit ähnlichen Eigenschaften einander benachbart. In Abb. 2.14 ist ein einfaches Beispiel eines zweidimensionalen Merkmalsraums gezeigt, der durch die Fläche $A$ und die Exzentrizität $\epsilon$ der in der Blasenfeldaufnahme erkannten Objekte aufgespannt wird. In diesem Beispiel haben ca. 97\% der Objekte eine Fläche kleiner als 60 Pixel und eine Exzentrizität größer als 0.96. Nur drei Objekte weisen eine deutlich größere Fläche oder eine geringere Exzentrizität auf. Der Vergleich mit Abb. 2.13 (b) und (d) zeigt, daß es sich bei einem der auffälligen Objekte um das Zentrum des Blasenclusters handelt, in dem einzelne Blasen nicht mehr aufgelöst werden können. Das zweite Objekt ist vermutlich eine stark defokussiert abgebildete Blase oder eine Abschattung an der Küvettenwand. Das dritte auffällige Objekt ist ein Artefakt, das durch die Abbildung zweier dicht benachbarter Blasen und einen niedrigen Segmentierungsschwellwert entstanden ist. Beide Blasen erscheinen als ein einziges, nahezu elliptisches Objekt. Objekte, deren Grauwerte nahe der Detektionsschwelle lie- 
gen, können i.d.R. nicht mehr zuverlässig erkannt werden. In Abb. 2.14 sind alle Objekte mit einer Fläche von weniger als 15 markiert.

Objekte, die auffällige Merkmale aufweisen, werden von der weiteren Analyse nicht ausgeschlossen, sondern markiert und gesondert behandelt. Beispielsweise können die Bilder zweier Blasen, die durch den Segmentierungvorgang fälschlicherweise als ein Objekt erkannt werden, durch die Anwendung morphologischer Operatoren voneinander getrennt werden. Neben den geometrischen Eigenschaften werden die Objekte durch ihre Bewegung charakterisiert. Die Bewegungsanalyse kann daher einen Hinweis darüber liefern, ob es sich bei sehr kleinen Objekten um rauschbedingte Fluktuationen oder um eine Blase nahe der Detektionsschwelle handelt.

Die auf der Grundlage des binären digitalen Bildes durchgeführte Objekterkennung und Klassifizierung liefert eine erste Abschätzung der Lage und der geometrischen Eigenschaften der Blasen. Für viele Anwendungen mag diese Genauigkeit bereits ausreichen. Eine auf der bisher dargestellten Methode fußende, modellbasierte Analyse der Grauwertstruktur erlaubt darüber hinaus eine subpixelgenaue Bestimmung der Objekteigenschaften. Diese wurde für die subpixelgenaue Auswertung der Messdaten im Rahmen dieser Arbeit durchgeführt.

\subsubsection{Bewegungsanalyse}

Eine Bildsequenz ist ein dreidimensionales Orts-Zeit-Bild. Die Bewegung eines Objektes entspricht einer Trajektorie in diesem Raum. Die Analyse dieser Bewegung kann - ebenso wie die Analyse eines einzelnen Bildes - objektbezogen oder grauwertorientiert sein. In diesem Abschnitt wird daher das explizite Verfolgen von Objekten mit einen Tracking-Algorithmus als Bestandteil der PTV - ebenso wie die Bewegungsanalyse mit Hilfe der Struktur-TensorMethode beschrieben.

Tracking-Algorithmus Die fundamentale Definition der Geschwindigkeit $\mathbf{u}$ eines Objektes ist gegeben durch

$$
\mathbf{u}(\mathbf{x}, t)=\frac{\Delta \mathbf{x}(\mathbf{x}, t)}{\Delta t},
$$

wobei das Objekt zum Zeitpunkt $t$ am Ort $\mathbf{x}$ befindet und $\boldsymbol{\Delta} \mathbf{x}$ die nach einem kurzen Zeitintervall $\Delta t$ beobachtete Ortsverschiebung bezeichnet. Das Ziel eines Tracking-Algorithmus ist, diese Verschiebungen der Objekte zu verfolgen und so ihre Trajektorien zu bestimmen. Es müssen also Objekten, die in einem Bild zum Zeitpunkt $t$ gefunden wurden, Objekte im zum Zeitpunkt $t+\Delta t$ folgenden Bild zugeordnet werden. Dies wird als Korrespondenzproblem bezeichnet und ist i.a. nicht eindeutig lösbar. Particle-Tracking-Algorithmen gehen daher üblicherweise von den folgenden heuristischen Annahmen aus:

- Es gibt eine maximale Geschwindigkeit $\left|\mathbf{u}_{\max }\right|$ für die Bewegung der Objekte. Ein Objekt kann daher in einem Zeitintervall $\Delta t$ maximal die Strecke $\left|\mathbf{u}_{\text {max }}\right| \Delta t$ zurücklegen. Es wird angenommen, daß diese Strecke klein ist gegenüber dem mittleren Objektabstand.

- Die Änderung der Geschwindigkeit ist klein, d.h. die Beschleunigung der Objekte kann vernachlässigt werden.

- Objekte, die sich in einer Region befinden, zeigen eine ähnliche Bewegung (common motion).

- Zwei Objekten in einem Bild entsprechen üblicherweise zwei Objekte im darauffolgenden Bild (consistent match). 
Für PTV-Algorithmen gelten die folgenden, allgemeinen Grundanforderungen:

- Robustheit. Der Algorithmus soll Trajektorien auch dann noch verfolgen können, wenn Objekte in einzelnen Bildern nicht enthalten sind. Dies ist möglich, wenn die Grauwerte des Objektes nahe der Detektionsschwelle liegen und damit nicht mehr zuverlässig in jedem Bild erkannt werden können.

- Numerische Effizienz. Die große Zahl der zu verfolgenden Trajektorien macht eine effiziente Implementierung notwendig.

In Abb. 2.15 ist eine schematische Darstellung eines 4-Bild-PTV-Algorithmus gezeigt [28]. Ein Objektpunkt $\mathbf{x}_{i}$ im ersten Bild wird als Startpunkt der Trajektorie gewählt. Um dieses Objekt wird im zweiten Bild ein Suchgebiet $S_{2}$ gelegt, dessen Radius durch die maximale Weglänge $\mathbf{u}_{\max } \Delta t$ bestimmt wird, die ein Objekt im Zeitintervall $\Delta t$ zurücklegen kann. Die Radien der Suchgebiete $S_{3}$ und $S_{4}$ in den Bildern 3 und 4 werden in ähnlicher Weise abgeschätzt, wobei üblicherweise $S_{2} \leq S_{3} \leq S_{4}$ gewählt wird. Es werden nun die im Suchgebiet $S_{2}$ in Bild 2 befindlichen Objekte festgestellt, von denen jedes eine mögliche Fortsetzung der Trajektorie darstellt. Eines dieser Objekte wird ausgewählt und mit $\mathbf{x}_{j}$ bezeichnet. Um die Fortsetzung der Trajektorie in Bild 3 zu finden, wird ein Suchgebiet $S_{3}$ in Bild 3 bestimmt, dessen Mittelpunkt durch lineare Extrapolation festgelegt wird. Alle Objekte in diesem Suchgebiet sind wiederum mögliche Fortsetzungen der Trajektorie. Von diesen Objekten wird ein Objekt gewählt und mit $\mathbf{x}_{k}$ bezeichnet. Und schließlich wird in Bild 4 ein Suchgebiet $S_{4}$ und die darin enthaltenen Objekte bestimmt. Eines der in $S_{4}$ enthaltenen Objekte wird als Endpunkt $\mathbf{x}_{l}$ der Trajektorie ausgewählt. Die Menge aller in den Suchgebieten $S_{2}, S_{3}$ und $S_{4}$ befindlichen Objekte bestimmen die möglichen Trajektorien $\left\{\mathbf{x}_{i}, \mathbf{x}_{j}, \mathbf{x}_{k}, \mathbf{x}_{l}\right\}$ mit dem gemeinsamen Startpunkt $\mathbf{x}_{i}$. Die Koordinaten bilden eine sich baumartig verzweigende Struktur. Aus dieser Schar möglicher Trajektorien wird diejenige als wahrscheinliche Trajektorie ausgewählt, deren Gesamtvarianz $\sigma_{t}$ minimal wird. Diese Gesamtvarianz einer Trajektorie wird aus der Varianz $\sigma_{l}$ der Länge

$$
\sigma_{l}=\sqrt{\frac{1}{3}\left(\left|\mathbf{d}_{i j}-\mathbf{d}_{m}\right|^{2}+\left|\mathbf{d}_{j k}-\mathbf{d}_{m}\right|^{2}+\left|\mathbf{d}_{k l}-\mathbf{d}_{m}\right|^{2}\right)} .
$$

und der Varianz der Winkel $\sigma_{\Theta}$

$$
\sigma_{\Theta}=\sqrt{\frac{1}{2}\left(\left(\Theta_{i k}-\Theta_{m}\right)^{2}+\left(\Theta_{j l}-\Theta_{m}\right)^{2}\right)}
$$

bestimmt. Für die Gesamtvarianz $\sigma_{t}$ gilt dann

$$
\sigma_{t}=\sqrt{\frac{\sigma_{l}^{2}}{\left|\mathbf{d}_{m}\right|^{2}}+\sigma_{\Theta}} .
$$

In den Gleichungen (2.40), (2.41) und (2.42) werden die folgenden Bezeichnungen für die relativen Abstände und Winkel verwendet:

$$
\mathbf{d}_{m}=\frac{1}{3}\left(\mathbf{d}_{i j}+\mathbf{d}_{j k}+\mathbf{d}_{k l}\right), \quad \Theta_{m}=\frac{1}{2}\left(\Theta_{i k}+\Theta_{j l}\right) \quad \text { und } \quad \mathbf{d}_{i j}=\mathbf{x}_{i}-\mathbf{x}_{j} \quad \text { usw. }
$$

Es bezeichnen $\Theta_{i k}$ den Winkel zwischen $\mathbf{d}_{i j}$ und $\mathbf{d}_{j k}$ und $\Theta_{j l}$ den Winkel zwischen $\mathbf{d}_{j k}$ und $\mathbf{d}_{k l}$. Mit $\mathbf{x}_{i}, \mathbf{x}_{j}, \mathbf{x}_{k}$ und $\mathbf{x}_{l}$ sind durchgehend jeweils die Orte von Objekten in den Bildern 1, 2, 3 und 4 bezeichnet.

Den Kollisionen der Blasen entsprechen von verschiedenen Startpunkten ausgehende und - im Sinne des vorgestellten Algorithmus - akzeptierte Trajektorien, die gemeinsame Objekte in den folgenden Bildern enthalten. 


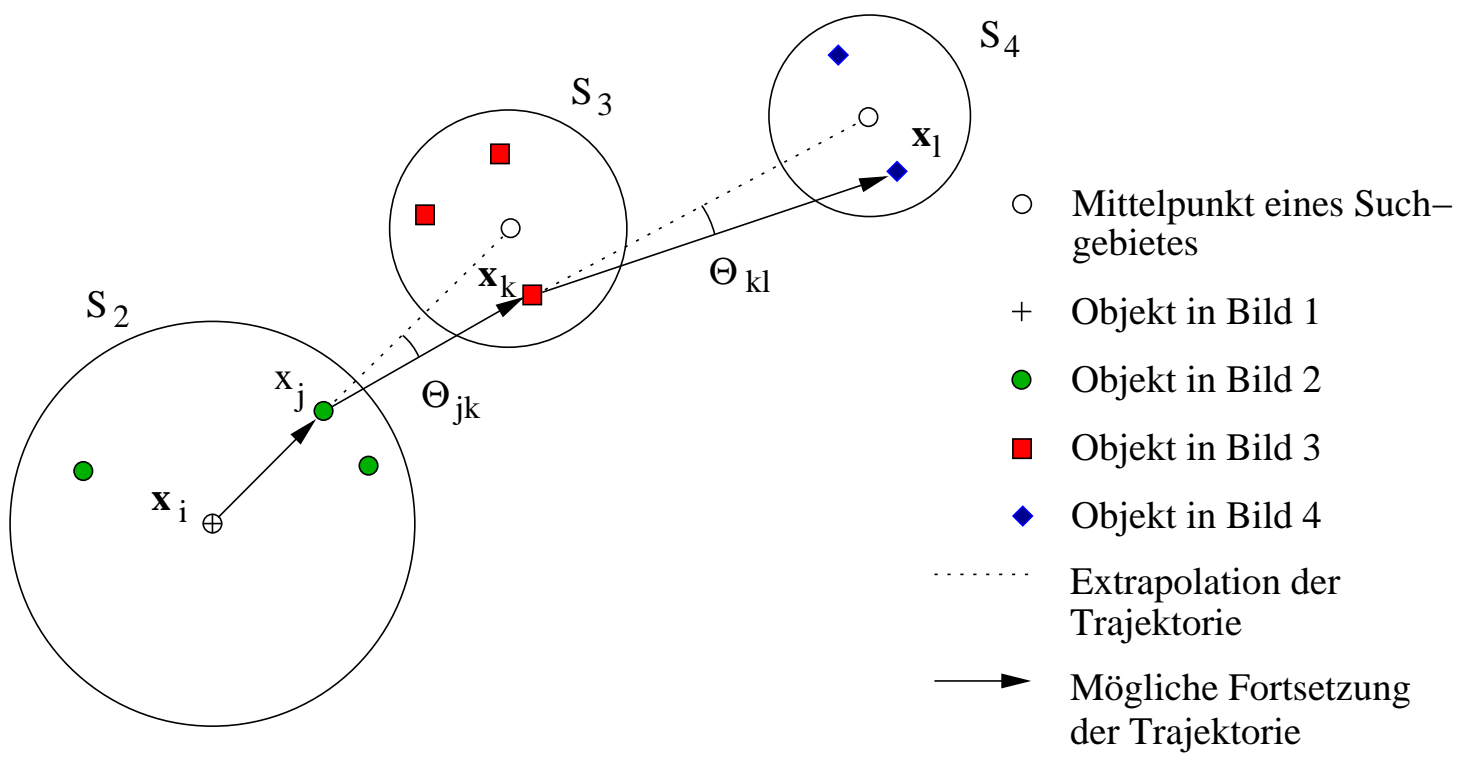

Abbildung 2.15.: Schematische Darstellung eines 4-Bild-Tracking-Algorithmus [28].

Struktur-Tensor-Methode Die Verallgemeinerung des Strukturtensors Gl. (2.29) auf drei Dimensionen $(x, y, t)$ zur Analyse von Bildsequenzen ist möglich [34]. Zur Interpretation der Resultate muß jedoch beachtet werden, daß das Bewegungsfeld und der durch die Bewegung der Objekte hervorgerufene optische Fluß nicht notwendig übereinstimmen. Für die hier diskutierten experimentellen Bedinungen ist dies jedoch in guter Näherung erfüllt. Der Strukturtensor kann definiert werden durch den symmetrischen Tensor

$$
\underline{\underline{\mathbf{J}}}=\left(\begin{array}{ccc}
J_{x x} & J_{x y} & J_{x t} \\
J_{x y} & J_{y y} & J_{y t} \\
J_{x t} & J_{y t} & J_{t t}
\end{array}\right)
$$

wobei $J_{p, q}$ mit $p, q \in\{x, y, t\}$ durch

$$
J_{p, q}(\mathbf{x}, t)=\int w\left(\mathbf{x}-\mathbf{x}^{\prime}, t-t^{\prime}\right) \partial_{p} g\left(\mathbf{x}^{\prime}, t^{\prime}\right) \partial_{q} g\left(\mathbf{x}^{\prime}, t^{\prime}\right) \mathrm{d} \mathbf{x} d t
$$

gegeben ist. Wie im zweidimensionalen Fall wird nun eine Eigenwertanalyse durchgeführt, die im Folgenden kurz skizziert wird. Es seien $\lambda_{1}, \lambda_{2}$ und $\lambda_{3}$ die Eigenwerte von $\underline{\mathbf{J}}$, wobei o.B.d.A. angenommen wird, daß $\lambda_{1} \geq \lambda_{2} \geq \lambda_{3}$ gilt. Die Bestimmung des optischen Flusses ist äquivalent zu der Bestimmung des Eigenvektors zum kleinsten Eigenwert $\lambda_{3}$.

Lokale Strukturen können nun mit Hilfe der Eigenwerte des Tensors Gl. (2.43) klassifiziert werden. Hierdurch ist eine effiziente Implementierung der tensorbasierten Bewegungsanalyse möglich. Folgende Fälle können unterschieden werden:

- Konstanter Grauwert: $\lambda_{1}=\lambda_{2}=\lambda_{3}=0$.

- Räumliche Orientierung und konstante Bewegung: $\lambda_{1}>0$ und $\lambda_{2}=\lambda_{3}=0$.

- Verteilte räumliche Strukturen und konstante Bewegung: $\lambda_{1}, \lambda_{2}>0$ und $\lambda_{3}=0$.

- Verteilte räumliche Strukturen und nichtkonstante Bewegung: $\lambda_{1}, \lambda_{2}, \lambda_{3}>0$. 
Desweiteren können aus dem Struktur-Tensor von der räumlichen Orientierung und der Geschwindigkeit unabhängige rotationsinvariante Parameter zur Beschreibung der Grauwertstruktur bestimmt werden. Gewählt werden hierfür das Bestimmtheitsmaß

$$
c_{c}=J_{x x}+J_{y y}
$$

das räumliche Kohärenzmaß

$$
c_{s}=\frac{\left(J_{y y}-J_{x x}\right)^{2}+4 J_{x y}^{2}}{\left(J_{y y}+J_{x x}\right)^{2}}
$$

und das totale Kohärenzmaß

$$
c_{t}=\left(\frac{\lambda_{1}-\lambda_{3}}{\lambda_{1}+\lambda_{3}}\right)^{2}
$$

Für eine Analyse des Struktur-Tensors-Verfahrens bzgl. statistischer und systematischer Fehler, inbesondere bzgl. seiner Robustheit gegenüber systematischen Fehlern durch Rauschen, ungleichmäßiger Bewegung, Bewegungsdiskontinuität und Beleuchtungsänderung wird auf die ausführliche Diskussion in [34] verwiesen.

Die Modellierung der Streak-Funktion In diesem Abschnitt wird die Analyse von Objekten aus Streulichtaufnahmen über die Streak-Funktion vorgestellt. Diese ermöglicht die Bewegungsanalyse durch die Analyse von einzelnen Bildern einer Sequenz. Ein Ausschnitt aus einer solchen Aufnahme ist in Abb. 2.16 (a) gezeigt. Durch die Bewegung des Objektes entsteht eine unscharfe orientierte Struktur, die als Streak bezeichnet wird. Aus der Bewegungsunschärfe können Informationen über die Geschwindigkeit des Objektes, d.h. Orientierung und Betrag, gewonnen werden. Diese modellbasierte Analyse der Bilddaten wird im Folgenden vorgestellt.

Die Grauwertverteilung für ein ruhendes Objekt sei gegeben durch eine Funktion $g_{\sigma}$, die nur vom Abstand vom Schwerpunkt $\mu$ abhängt:

$$
g_{\sigma}(\mathbf{x})=g_{\sigma}(|\mathbf{x}-\mu|) \quad \text { mit } \quad \mu=\frac{\int_{-\infty}^{\infty} \mathbf{x} g_{\sigma}(\mathbf{x}) d^{2} x}{\int_{-\infty}^{\infty} g_{\sigma}(\mathbf{x}) \mathrm{d}^{2} x} .
$$

Es wird angenommen, daß $g_{\sigma}$ als zweidimensionale Gaussverteilung

$$
g_{\sigma}(\mathbf{x})=\frac{1}{2 \pi \sigma^{2}} \exp \left(-\frac{1}{2}\left(\frac{\mathbf{x}}{\sigma}\right)^{2}\right)
$$

mit Standardabweichung $\sigma$ gewählt werden kann. Das Objekt bewege sich nun auf einer Trajektorie $\mathbf{s}(t)$. Die Zeitpunkte $t_{0}$ und $t_{1}$ bezeichnen Beginn und Ende der Belichtung mit Dauer $t_{B}=t_{1}-t_{0}$. Die hieraus resultierende Grauwertverteilung ist daher gegeben durch das Integral über die Belichtungszeit $t_{B}$

$$
g_{s}(\mathbf{x})=\frac{1}{t_{B}} \int_{t_{0}}^{t_{1}} g_{\sigma}(\mathbf{x}-\mathbf{s}(t)) \mathrm{d} t .
$$

Die Bahnkurve $\mathbf{s}(t)$ kann nun in eine Potenzreihe um $\mathbf{s}_{0}=\mathbf{s}\left(t=t_{0}\right)$ entwickelt werden:

$$
\mathbf{s}(t)=\mathbf{s}_{0}+\frac{\partial \mathbf{s}}{\partial t}\left(t_{0}-t\right)+\frac{1}{2} \frac{\partial^{2} \mathbf{s}}{\partial t^{2}}\left(t_{0}-t\right)^{2}+\mathcal{O}\left(\left(t_{0}-t\right)^{3}\right) .
$$

Für eine Bewegung mit konstanter Geschwindigkeit $\mathbf{v}_{0}$ ist die Bahnkurve gegeben durch

$$
\mathbf{s}(t)=\mathbf{s}_{0}+\mathbf{v}_{0}\left(t_{0}-t\right) \quad \text { mit } \quad \mathbf{v}_{0}=\frac{\partial \mathbf{s}}{\partial t}
$$


(a)

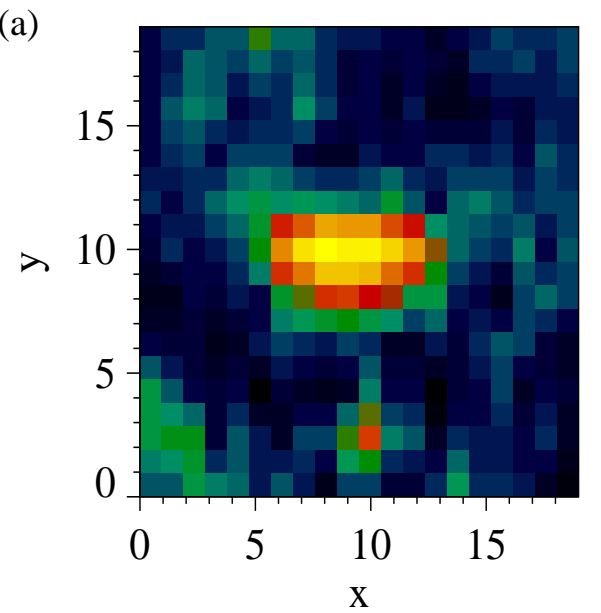

(d)

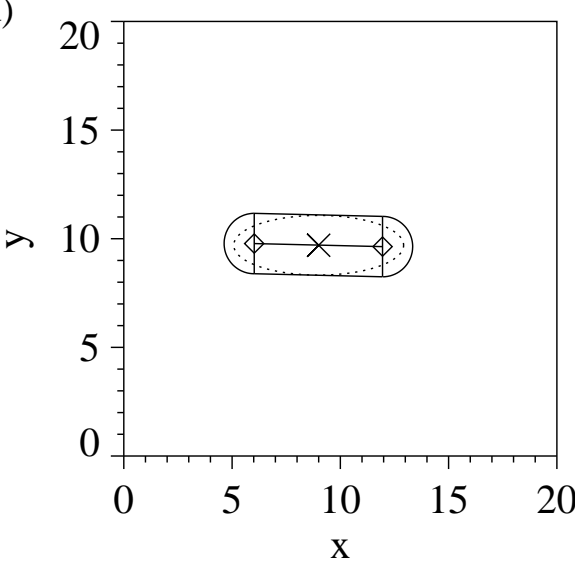

(b)

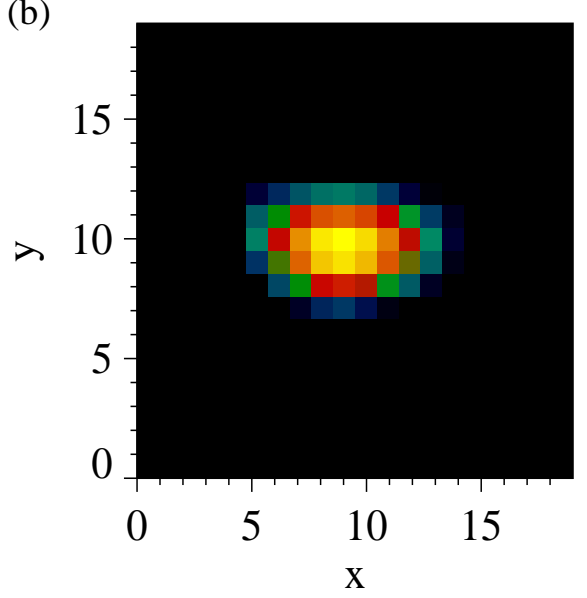

(c)

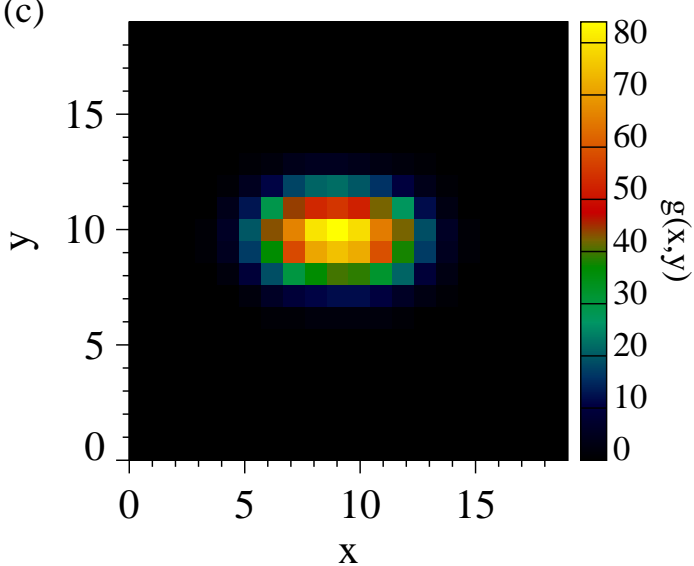

Abbildung 2.16.: Modellierung eines Streaks. (a) Ausschnitt der Grauwertverteilung einer Streulichtaufnahme. (b) Gefilterte Grauwertverteilung. (c) Anpassung der Funktion Gl. 2.53 zur Bestimmung der Modellparameter. (d) Ergebnis der Anpassung. Mit $\times$ ist der Schwerpunkt des segmentierten Bildes markiert und die gestrichelte Ellipse ist aus den Momenten der Verteilung bestimmt. Die Rauten markieren Anfangs- und Endpunkt der Bewegung. Die durchgezogenen Linien zeigen den Verlauf des Streaks mit Halbwertsbreite $\sigma$. 
Daher folgt für die Grauwertverteilung $g_{\sigma}$

$$
g_{\sigma}^{(1)}=\frac{1}{\sqrt{2 \pi} 2 \mathbf{a}^{2} \sigma} \exp \left(-\frac{\mathbf{a}^{2} \mathbf{b}^{2}-(\mathbf{a b})^{2}}{2 \mathbf{a}^{2} \sigma^{2}}\right)\left(\operatorname{Erf}\left(\frac{\mathbf{a}^{2}-\mathbf{a b}}{\sqrt{2}|\mathbf{a}| \sigma}\right)+\operatorname{Erf}\left(\frac{\mathbf{a b}}{\sqrt{2}|\mathbf{a}| \sigma}\right)\right)
$$

mit $\mathbf{a}=\mathbf{s}_{1}-\mathbf{s}_{0}$ und $\mathbf{b}=\mathbf{x}-\mathbf{s}_{0}$. Mit Erf wird die durch das Integral der Gaussverteilung

$$
\operatorname{Erf}(z)=\frac{2}{\sqrt{\pi}} \int_{0}^{z} \exp \left(-z^{\prime 2}\right) \mathrm{d} z^{\prime}
$$

definierte Fehlerfunktion bezeichnet. Für eine Bewegung mit konstanter Beschleunigung $\mathbf{a}_{0}$ gilt entsprechend

$$
\mathbf{s}(t)=\mathbf{s}_{0}+\mathbf{v}_{0}\left(t_{0}-t\right)+\frac{1}{2} \mathbf{a}_{0}\left(t_{0}-t\right)^{2} \quad \text { mit } \quad \mathbf{a}_{0}=\frac{\partial^{2} \mathbf{s}}{\partial t^{2}}
$$

Dies führt zu einer Grauwertverteilung $g_{\sigma}^{(2)}$ eines durch eine beschleunigte Bewegung hervorgerufenen Streaks, die hier jedoch nicht explizit angegeben wird.

Die Analyse der Grauwertstruktur wird anhand der in Abb. 2.16 (a-d) gezeigten Aufnahme exemplarisch durchgeführt. Das Originalbild (a) wird zunächst gefiltert und von inhomogenem Hintergrund befreit. Dieses Bild wird segmentiert und der Streak durch seinen Schwerpunkt und weitere Formparameter charakterisiert. Aus den Momenten des segmentierten Bildes können die Orientierung sowie die Breite und die Länge des Streaks abgeschätzt werden. Diese Werte werden als Startwerte für die nichtlineare Regression verwendet, um ihre rasche Konvergenz zu gewährleisten. Die Grauwertverteilung der Modellfunktion ist in Abb. 2.16 (c) in Falschfarbendarstellung und die Start- und Endpunkte der Bewegung des Objektes in Abb. 2.16 (d) dargestellt.

\subsection{Radienmessung}

Ein charakteristischer Parameter eines Kavitationsblasenfeldes ist die Verteilung der Ruheradien. Zur Messung dieser Größe wurden in der Literatur verschiedene optische und akustische Verfahren vorgestellt [21, 13]. In diesem Kapitel wird ein Verfahren zur optischen Messung der Blasenradien in akustischen Kavitationsblasenfeldern vorgestellt.

Methode Da die Blasen im Schallfeld oszillieren, wird zum Zeitpunkt $t$ der instantane Radius $R(t)$ gemessen. Dieser Radius ist eine Funktion des Ruheradius $R_{0}$, der Amplitude $p_{a}$ und der Frequenz $f$ des anregenden Schallfeldes. Die Abhängigkeit der radialen Dynamik der Blase von diesen Größen kann durch ein Blasenmodell theoretisch beschrieben werden [37, 63]. Die Gültigkeit dieser Modelle ist experimentell verifiziert. Sind die Frequenz und die Amplitude des Schallfeldes bekannt, so liefert das Modell der Blasenschwingung eine nichtlineare Funktion, die den instantanen Blasenradius $R(t)$ auf den Blasenruheradius $R_{0}$ abbildet [57]. Ein Beispiel für eine solche Abbildung ist in Abb. 2.17 für verschiedene Anregungsamplituden gezeigt. Aus dieser Darstellung ist ersichtlich, daß für kleine Ruheradien $R_{0}$ bis zu einem kritischen $R_{0, B}$ keine signifikante Oszillation zu beobachten ist. Diese Grenze wird als Blake-Schwelle bezeichnet. Für Blasen mit Ruheradien $R_{0}>R_{0, B}$ schwingen die Blasen deutlich auf; für sehr große Ruheradien hingegen ist eine Sättigung der maximalen Schwingungsamplitude $R_{\max }$ zu verzeichnen. Mit Hilfe der Abb. 2.17 (a) kann eine gemessene Radienverteilung in eine Verteilung der Ruheradien umgerechnet werden. In Abb. 2.17 (b) ist für die Messung diejenige Phasenlage gewählt 
(a)

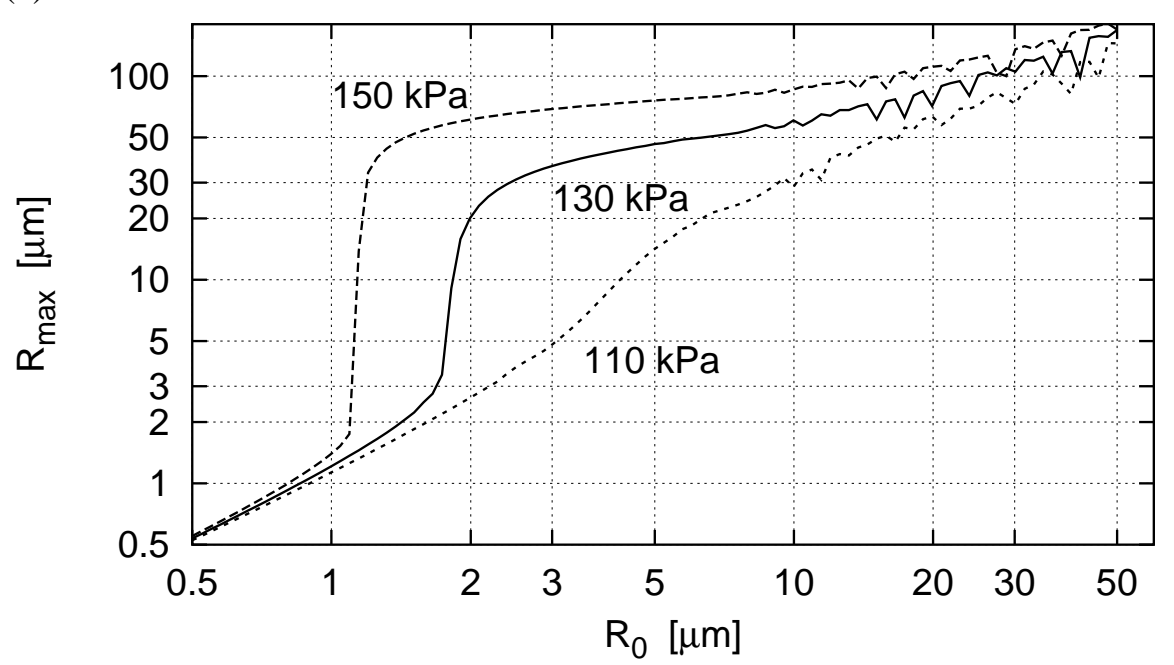

(b)

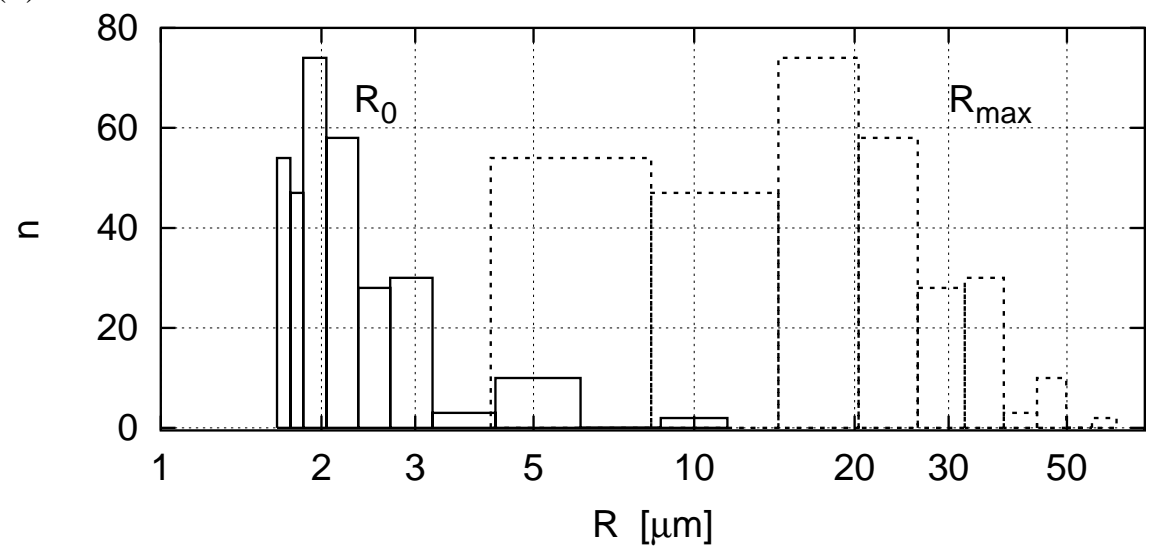

Abbildung 2.17.: (a) Maximalradien $R_{\max }$ der Blasen als Funktion der Ruheradien $R_{0}$ für $p_{a}=110,130$ und $150 \mathrm{kPa}$ und $f=20 \mathrm{kHz}$. (b) Beispiel der Bestimmung der Ruheradien $R_{0}$ aus einer gemessenen Verteilung der Maximalradien $R_{\max }$.

worden, für die die Blasen ihren maximalen Radius annehmen. Das beschriebene Verfahren zur Ruheradienmessung kann nun für eine allgemeine Wahl der Phasenlage der Radienmessung verallgemeinert werden. Für jede Phasenlage ergibt sich eine charakteristische Verteilung der instantanen Radien $R(t)$. Für eine gegebene gemessene Verteilung kann nun die sie erzeugende Ruheradienverteilung bestimmt werden. Es wird dabei vorausgesetzt, daß diese Verteilung über die Dauer der Messung stationär ist. Da der Anregungsdruck $p_{a}=p_{a}(\mathbf{x})$ einen entscheidenden Einfluß auf die Bestimmung der Ruheradien hat, liefert die Auswertung der Messung eine ortsaufgelöste Verteilung der Ruheradien. In Abb. 2.18 ist die Darstellung der Abb. 2.17 (a) für verschiedene Phasen verallgemeinert worden. Die Darstellung der Abb. 2.17 (a) ergibt sich dann für eine feste Phase als vertikaler Schnitt durch das Diagramm (Abb. 2.18 (1.b), (2.b) und (3.b)). Mit $R_{s}$ der Grenzradius für Oberflächeninstabilitäten bezeichnet (vgl. Abb. 2.19).

Die Genauigkeit des vorgestellten Meßverfahrens zur Bestimmung der Ruheradien ist begrenzt durch die Genauigkeit, mit der Druck- und Ortsmessungen durchgeführt werden können. Druckmessungen in kavitierenden Flüssigkeiten sind mit erheblichen Schwierigkeiten verbunden. Kriterien für die Auswahl des Hydrophons ist der beabsichtigte Druckmeßbereich von ca. 1- 

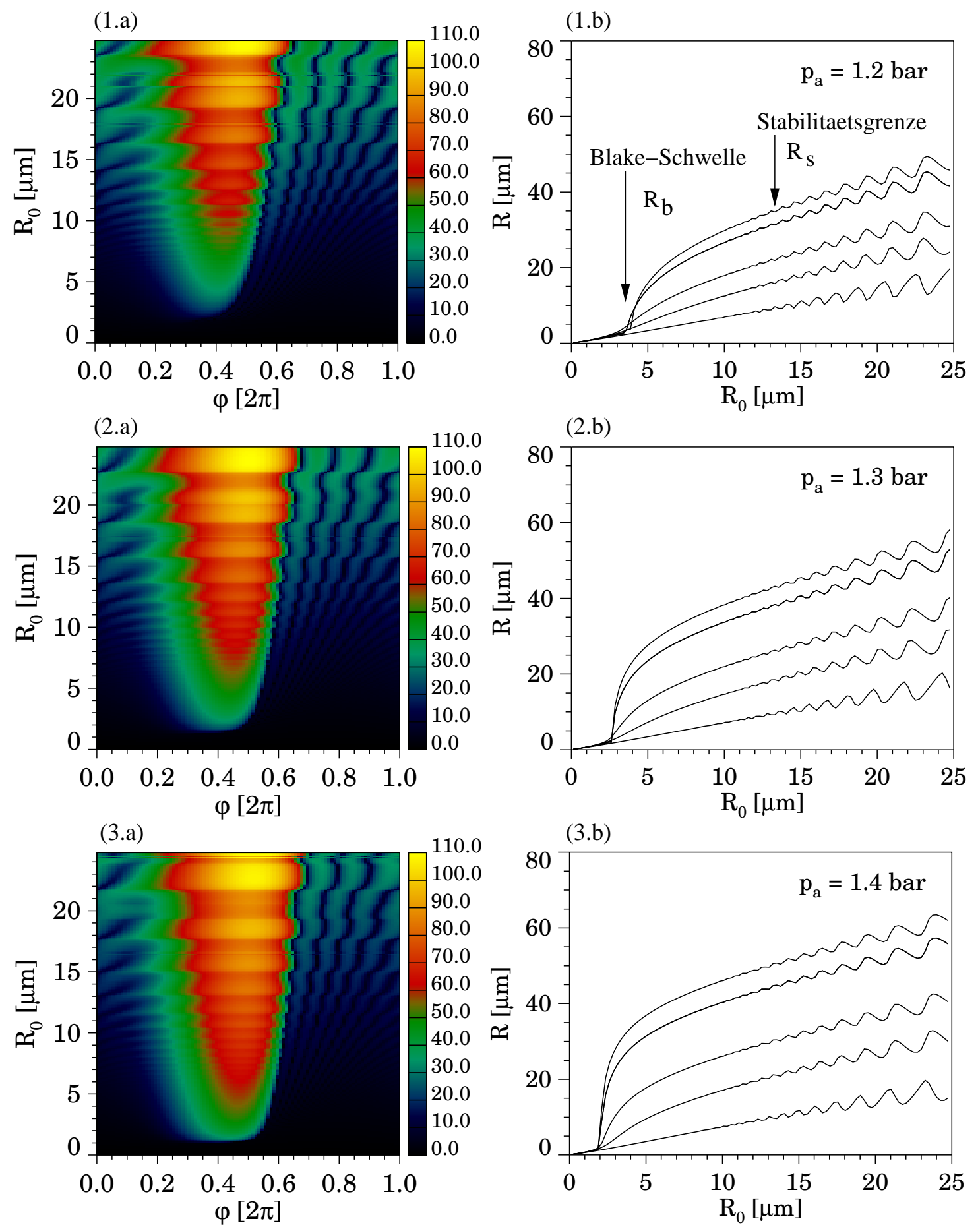

Abbildung 2.18.: (1.a), (2.a) und (3.a): Blasenradien $R(t)$ als Funktion der Ruheradien $R_{0}$ und Phase $\varphi$ für $p_{a}=1.2,1.3$ und 1.4 bar und einer Anregungsfrequenz von $f=21$ kHz. (1.b), (2.b) und (3.b): Vertikale Schnitte durch diese Darstellung für die Phasen $\varphi=\{0.05,0.1,0.15,0.2,0.225\}$. Die Blake-Schwelle $R_{B}$ und die Stabilitätsgrenze $R_{s}$ für Oberschwingungen sind markiert. 


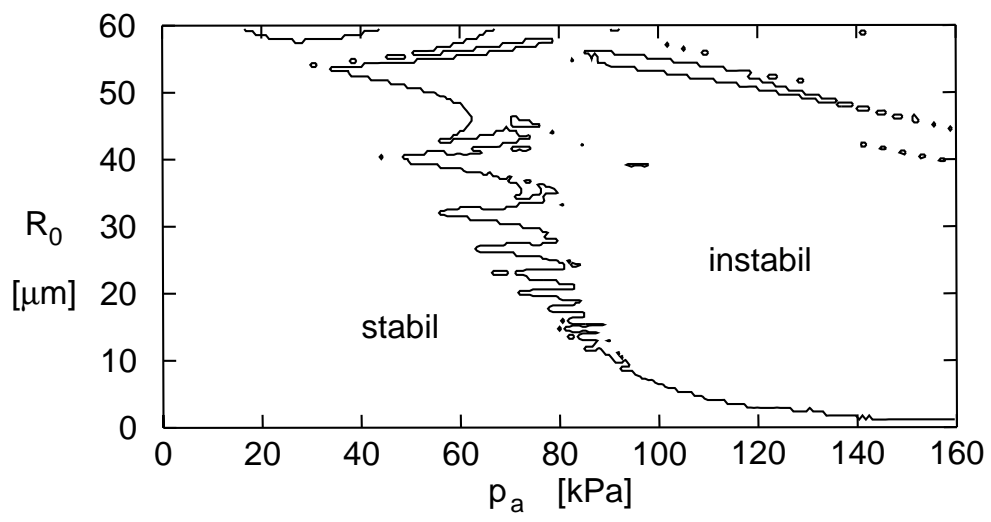

Abbildung 2.19.: Theoretische Stabilitätsgrenze für Oberflächeninstabilitäten in der durch den Anregungsdruck $p_{a}$ und den Ruheradius $R_{0}$ aufgespannten Parameterebene. Das hier dargestellte Beispiel ist für eine Anregungsfrequenz von $f=20 \mathrm{kHz}$ berechnet worden [57].

3 bar im Frequenzbereich der Anregung von $\approx 20-25 \mathrm{kHz}$. Die Abmessungen der Küvette von etwa $5 \mathrm{~cm}$ Kantenlänge und eine möglichst hohe Ortsauflösung schränken die Auswahl des Hydrophons weiter ein. Die Druckmessungen wurden in dieser Arbeit durchgeführt mit einem Hydrophon Bruel \& Kjaer 8103.

\subsection{Zusammenfassung}

In diesem Kapitel wurden die experimentellen Methoden zur Untersuchung akustischer Kavitationsblasenfelder vorgestellt und diskutiert. Ausgangspunkt hierfür war eine Übersicht über experimentelle Verfahren der Strömungsmechanik. Da die Kenntnis der makroskopischen Bewegung des Blasenfeldes die Kenntnis der Bewegung der Einzelblase voraussetzt, wurde - als wesentliches Ergebnis dieses Kapitels - ein Particle-Tracking-Verfahren implementiert. Die Abfolge der Abschnitte in diesem Kapitel spiegelt das Flußdiagramm dieses Verfahrens wieder.

Grundlegend für dieses bildgebende Meßverfahren ist zunächst die Bildaufnahme. Die Anforderungen an die räumliche und zeitliche Auflösung werden von der verwendeten Hochgeschwindigkeits-CCD-Kamera HiSIS 2002 erfüllt. Die Abbildungseigenschaften und die Fehlerquellen der Bildaufnahme wurden diskutiert.

Der Bildaufnahme folgt die Bildbearbeitung und -analyse. Die Abfolge der Operationen der Bildverarbeitung und -analyse ist hierachisch gegliedert. Sie beginnt mit der Bildvorbearbeitung. In den digitalen Bildern werden dann in der Phase der Bildbearbeitung durch einen Segmentierungsalgorithmus die Blasen detektiert. Dieser Objekterkennung folgt in der Bildanalyse die Klassifizierung der Objekte mit Hilfe von invarianten Formparametern. Durch diese Klassifizierung ist es möglich, Abbildungsfehler und Artefakte der Abbildung aus der weiteren Analyse auszuschließen.

Im Folgenden wird dann die Bewegung analysiert. Die Bewegung eines Objektes in einer Bildsequenz wird als Bewegung in einem dreidimensionalen Orts-Zeit-Bild interpretiert. Die Verfolgung der Trajektorien der Objekte in diesem Raum wird mit dem Partikel-Tracking-Algorithmus oder durch die Bewegungsanalyse mit Hilfe des Struktur-Tensor-Verfahrens durchgeführt

Für das Verständnis der involvierten physikalischen Prozesse und die folgende Modellierung 
akustischer Kavitationsblasenfelder ist die Kenntnis der Ruheradienverteilung der Blasen von entscheidender Bedeutung. Es wurde ein Verfahren zur optischen Messung der instantanen Blasenverteilungen und die darauf basierende Methode zur Rekonstruktion der Ruheradienverteilungen vorgestellt und diskutiert.

Die Messung der Druckverteilung in einem Resonator stellt ein bisher - auch in der Literatur - nicht befriedigend gelöstes experimentelles Problem dar. Aufgrund der Notwendigkeit der detaillierten Kenntnis des Druckes für das Verständnis der dynamischen Eigenschaften des untersuchten Systems müssen hier zukünftig verstärkt Anstrengungen unternommen werden.

In dem nun folgenden Kapitel werden die experimentellen Daten vorgestellt und diskutiert, die mit Hilfe der hier dargestellten Meßmethoden gewonnen wurden. 


\section{Experiment: Meßergebnisse und Auswertung}

Dieses Kapitel beschreibt die experimentellen Beobachtungen der Strukturbildungsphänomene in akustischen Kavitationsblasenfeldern. Es beginnt in Abschn. 3.1 mit der phänomenologischen Beschreibung der beobachteten Strukturen und vollzieht am konkreten experimentellen Beispiel die in der Einleitung in Kapitel 1 dargestellten Skalenbetrachtungen nach. Auf der Grundlage der in Kapitel 2 erarbeiteten Meßverfahren werden in Abschn. 3.2 die experimentellen Fragestellungen formuliert. In den folgenden Abschnitten werden die Ergebnisse der Orts-, Geschwindigkeits- und Radienmessungen dargestellt und diskutiert. Aus diesen Daten können die Trajektorien der Blasen bestimmt und durch die Betrachtung der Kollisionsprozesse die mittlere Lebensdauer der Blasen abgeschätzt werden. Das Kapitel schließt mit einer Zusammenfassung.

\subsection{Phänomenologische Beschreibung}

Wir beginnen mit einer phänomenologischen Beschreibung der beobachteten makroskopischen Strukturen. In Abb. 3.1 ist ein akustisches Kavitationsblasenfeld gezeigt, wie es vom Beobachter mit bloßem Auge wahrgenommen werden kann. Der Resonator, in dem sich die Struktur ausbildet, wird in der (1,1,1)-Mode betrieben und die uns interessierende Struktur befindet sich am Orte des Druckbauchs des Stehwellenfeldes. Das Blasenfeld wird mit weißem Licht parallel zur Bildebene beleuchtet und so erscheinen die das Licht streuenden Blasen als helle Objekte vor dunklem Hintergrund.

Blasen entstehen in den Randzonen des Resonators, an Unebenheiten der Wände oder an in der Flüssigkeit schwebenden Kavitationskeimen. Aus diesen äußeren Raumbereichen bewegen sich die im Schallfeld schwingenden Blasen in das Zentrum der Struktur. Die räumliche Verteilung ist inhomogen: Es bilden sich Filamente aus sich in das Zentrum bewegenden Blasen. Diese Filamente weisen vielfach dendritische Verzeigungen auf, an denen die Filamente zusammenstoßen. Während der Schwerpunkt der Struktur stationär im Raum angeordnet erscheint, können bei den Filamenten Fluktuationen um eine mittlere Lage und Orientierung beobachtet werden.

Im Zentrum der Struktur, in welches alle Blasen von außen einströmen, ist ein feiner, hell leuchtender Nebel sichtbar, der aus sehr kleinen Blasen besteht. Die Dichte dieser Blasen nimmt nach außen hin rasch ab und die fluktuierende Intensität des gestreuten Lichtes ist ein Indiz für die vorherrschende, die Mikroblasen nach außen transportierende Flüssigkeitsströmung.

Die Bewegung einzelner Blasen kann mit dem bloßen Auge nicht verfolgt werden. Ähnlich dem durch die Trägheit des Auges hervorgerufenen Effekt, integriert bei der in Abb. 3.1 gezeigten Aufnahme die Kamera über die Dauer der Belichtungszeit. Erst durch diese Integration über das Streulicht einer Vielzahl von Blasen werden die Filamente sichtbar und die Struktur der Anordnung der Blasen als Eigenschaft wahrgenommen. Diese Beobachtung ist die phänomenologische 


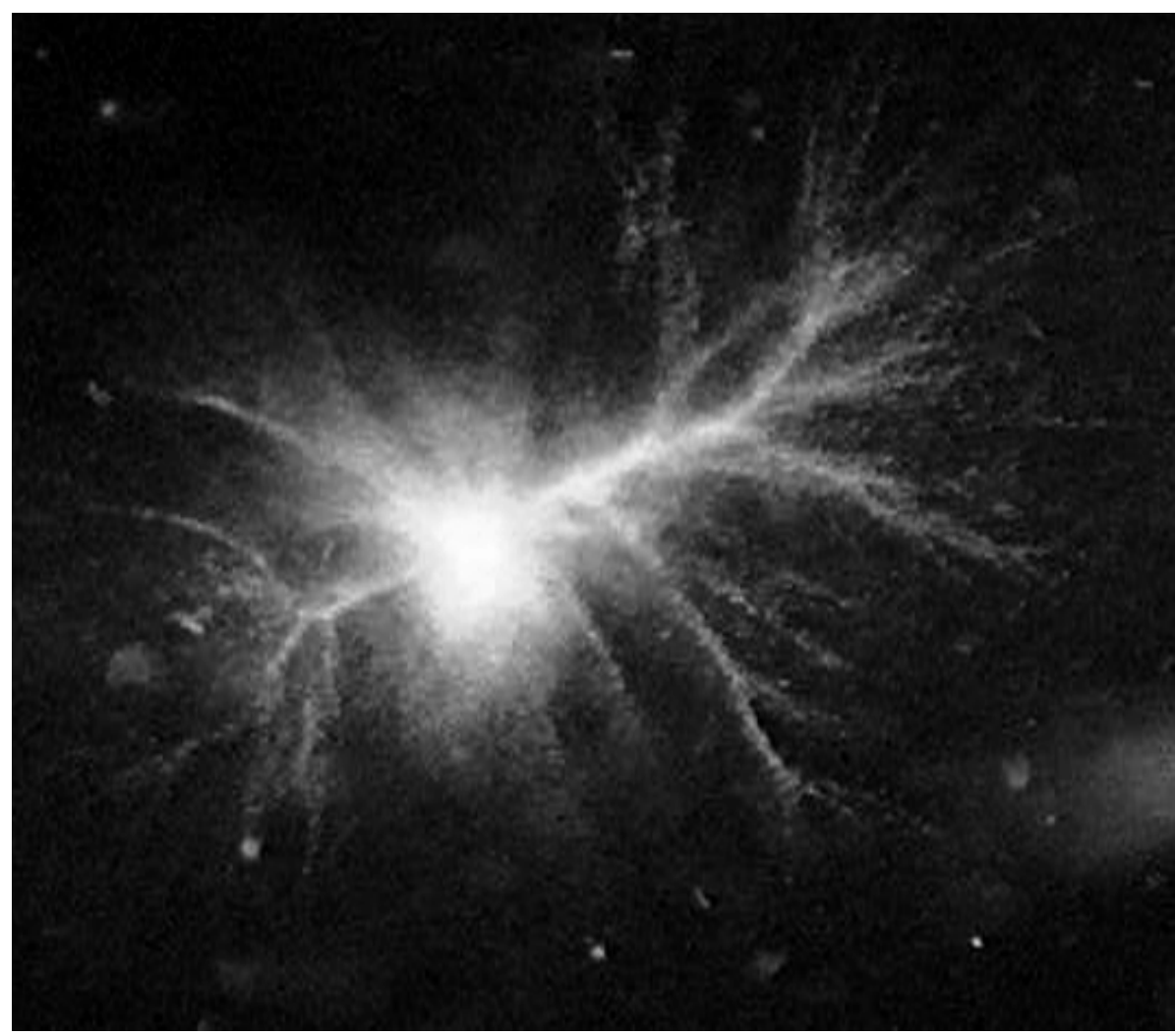

Abbildung 3.1.: Filamentstruktur in einem Stehwellenfeld. Die Aufnahme zeigt die Verteilung der Blasen im Streulicht. Die Blasen bewegen sich von den Randbereichen in den Druckbauch, der in der Bildmitte liegt. Durch die Belichtungszeit von 2ms wird über die Blasenverteilung integriert (Bildausschnitt $1.2 \times 1.6 \mathrm{~cm}^{2}$ ).

Beschreibung der in Kapitel 1 vorgestellten Skalenanalyse und zugleich ihre Motivation: Der Strukturbegriff bedarf als Grundlage der Definition der für das betrachtete physikalische System charakteristischen Raum- und Zeitskalen.

Ein anderes Bild des Blasenfeldes bietet sich dem Beobachter, wenn das Blasenfeld statt mit bloßem Auge oder einer Kamera mit einer Belichtungszeit von einigen Millisekunden mit einer Belichtungszeit von wenigen Mikrosekunden betrachtet wird. Ein Beispiel hierfür ist in Abb. 3.2 (a) gezeigt. Die Intensität des von den Blasen gestreuten Lichtes und die Empfindlichkeit der Sensoren der Kamera wären zu gering, um eine Abbildung des Blasenfeldes im Streulicht durchzuführen. Daher werden für diese Aufnahme Lichtquelle und Kamera auf einer Achse ausgerichtet und die Blasen im Durchlicht aufgenommen. Deutlich erkennt man die geradlinige Anordnung der von außen nach innen strömenden Blasen. Die unscharfe Abbildung der Blasen im Bildhintergrund vermittelt einen Eindruck von ihrer Anordnung im dreidimensionalen Raum.

Vergleicht man die Abb. 3.1 und 3.2 miteinander, so hat die Komplexität der Struktur in dieser Momentaufnahme der besseren Zeitauflösung entsprechend abgenommen. Die Vergrößerung eines Ausschnittes dieses Bildes offenbart noch mehr Detailstruktur. In Abb. 3.2 (b) sind einzelne Blasen unterschiedlicher Größe erkennbar, die einander in einem Filament folgen. Die Abstände der Blasen zueinander betragen offenbar wenige Blasenradien.

Diese Momentaufnahme liefert keinen Aufschluß über die Dynamik der Bewegung der Blasen. 

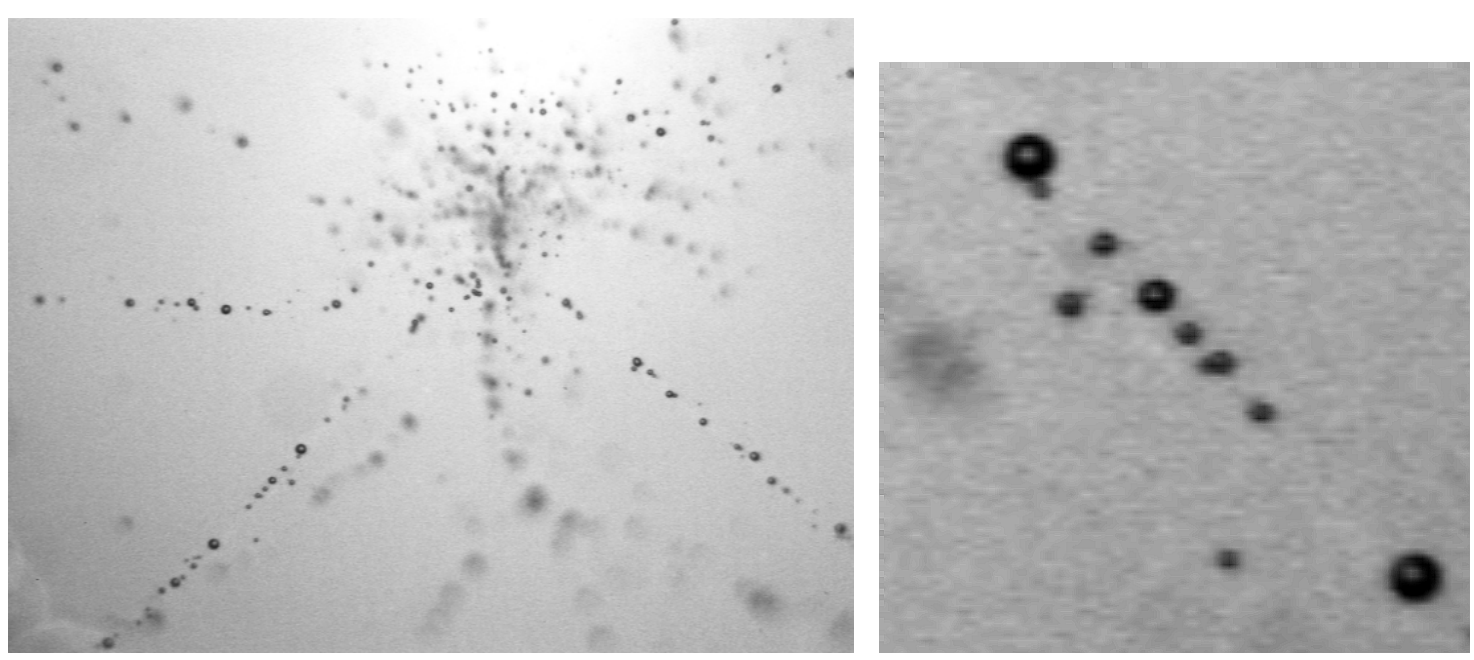

Abbildung 3.2.: (a) Anordnung der Blasen in filamentartigen Strukturen, in denen der mittlere Blasenabstand wenige Radien beträgt (Bildausschnitt ca. $6 \times 8 \mathrm{~mm}^{2}$ ). (b) Die Ausschnittvergrößerung zeigt die Ausrichtung der nahezu sphärischen Blasen. Typisch ist die eine vorauslaufende große Blase, der mehrere kleine Blasen folgen (Bildausschnitt ca. $1 \times 1 \mathrm{~mm}^{2}$, Belichtungszeit jeweils $3 \mu \mathrm{s}$ ).

Die Bewegung erst setzt Raum- und Zeitskala in ein Verhältnis und ist eine Eigenschaft, d.h. ein charakteristisches Merkmal der beobachteten Struktur.

\subsection{Methodik der Messungen}

Die in Kapitel 2 beschriebenen Verfahren erlauben die zeitaufgelöste Messung der Orte, Geschwindigkeiten und Radien der Blasen in einem Kavitationsblasenfeld. Mit einem TrackingAlgorithmus können die Orte der Blasen über eine Bildsequenz verfolgt und zu Trajektorien verknüpft werden. Hierdurch kann die zeitliche Entwicklung der Bewegung einzelner Blasen untersucht werden. Die Zeitauflösung der Meßapparatur erlaubt nicht, die Dynamik einer Blase im Verlaufe der Periode des anregenden Schallfeldes zu untersuchen. Lediglich in jeder achten Periode kann das Blasenfeld aufgezeichnet werden. Die Bewegung zwischen diesen Momenten bleibt uns hier verborgen. Allerdings kann sehr ausdauernd beobachtet werden: 16000 Einzelbildern mit einer Abtastrate von $2.25 \mathrm{kHz}$ entsprechen einer Dauer von etwa $10^{5}$ Perioden. Damit ist es möglich, eine statistische Beschreibung auf der Basis vieler Einzelereignisse zu geben.

Es werden in den Messungen folgenden Fragestellungen untersucht:

\section{- Bewegung einer Einzelblase.}

- Wechselwirkung mehrerer Blasen.

- Kollision und Fragmentierung von Blasen.

Die Untersuchung eines Blasenfeldes erlaubt eine statistische Beschreibung seiner Eigenschaften. Hierzu zählen insbesondere:

- Verteilung der Orte. 
- Verteilung der Geschwindigkeiten.

- Verteilung der Radien.

- Verteilung der Kollisionsereignisse.

\subsection{Auswertung der Messungen}

Die folgende Darstellung der Auswertung beschränkt sich auf die wesentlichen Meßergebnisse.

\subsubsection{Verteilung der Orte}

In diesem Abschnitt werden die Verteilungen der Orte der Blasen vorgestellt und diskutiert. Die Messungen wurden für verschiedene Anregungsamplituden und Phasenlagen der Anregung durchgeführt. Für die Darstellung der Ergebnisse werden zweidimensionale Histogramme erstellt, deren Bin-Größen einen Quadratpixel betragen. Die räumliche Auflösung der Ortsmessung beträgt in diesem Beispiel ca. 0.1 Pixel, was für das typische Meßvolumen einer absoluten Auflösung von $10 \mu \mathrm{m}$ entspricht. Wenn nicht anders erwähnt, so haben die ausgewerteten Bildsequenzen eine Länge von 2250 Bildern; dies entspricht einer Aufnahmedauer von einer Sekunde.

In Abb. 3.3 ist die Verteilung der Orte der Blasen in Abhängigkeit von der Phasenlage dargestellt. Bei einem konstantem Schalldruck von $75 \mathrm{kPa}$ und $f=21.5 \mathrm{kHz}$ wurden die acht Phasenlagen $\varphi=\left\{-19^{\circ}, 15^{\circ}, 58^{\circ}, 92^{\circ}, 146^{\circ}, 167^{\circ}, 213^{\circ}, 250^{\circ}\right\}$ gewählt. Wie zu erwarten war, ist die Häufigkeitsverteilung stark von der Phasenlage abhängig. Da Druck und Frequenz nicht verändert werden, ändern sich nicht die Art und die Häufigkeit, mit der Blasen erzeugt und durch Kollsionen vernichtet werden. Es werden lediglich unterschiedliche Ansichten eines ansonsten für die Dauer der Aufnahme als stationär betrachteten Systems gewonnen. In Abb. 3.3 (a,b) und $(\mathrm{c}, \mathrm{d})$ werden fast keine Blasen gemessen. Mit wachsender Phase nimmt die Häufigkeit über (c) bis (d) zu, erreicht in (e) ihr Maximum, um dann wieder abzusinken. Bemerkenswert ist, daß dabei zunächst die Population der Blasen in den Streamern zunimmt. Die Struktur der Streamer ist in Abb. 3.3 (d,f) ab deutlichsten zu erkennen. In Abb. 3.3 (e) haben die Blasen phasenbedingt ihre maximale Sichtbarkeit erreicht. In diesem Bild wird deutlich, daß sich, obwohl die Streamer eine von den Blasen bevorzugte Region darstellen, eine durchaus erhebliche Anzahl von ihnen in den übrigen Raumbereichen bewegen.

In Abb. 3.4 ist die Verteilung der Blasenpositionen für fünf verschiedene Anregungsamplituden dargestellt. Die Amplitude wächst von (a) bis (e). Deutlich erkennbar ist die für alle Werte der Amplitude inhomogene Verteilung der Blasen in den Filamenten. Für geringe Drücke zeigt Abb. $3.4(a, b)$ eine klare Struktur und eine eindeutige Lokalisierung der Blasen in den Streamern. Obwohl das Erscheinungsbild für jede Anregungsamplitude die gleichen Merkmale aufweist, ist die Detailstruktur unterschiedlich. Es gibt jeweils wenige, aber deutlich herausragende Filamente. Für höhere Amplituden hingegen wird diese Struktur verbreitert durch Blasen, die sich aus anderen Raumbereichen in das Zentrum bewegen.

Die folgende Tabelle gibt die Anzahl der Blasen an, die gleichzeitig auf einem Bild detektiert werden konnten:

\begin{tabular}{l|ccccc} 
Druck $[\mathrm{kPa}]$ & 75 & 80 & 120 & 130 & 140 \\
\hline Blasen pro Bild & 6 & 14 & 11 & 17 & 31
\end{tabular}


Die Anzahl der Blasen pro Bild steigt für wachsenden Anregungsdruck an. Lediglich für $p_{a}=$ 130 (Abb. 3.4 (c)) sinkt dieser Wert. Dies korrespondiert zu der Beobachtung, daß sich in diesem Bild der Schwerpunkt der Struktur aus dem Zentrum des Bildes nach links verschoben hat.

Die Verteilung der Blasen in den Filamenten kann durch den Abstand der Blasen zu ihren nächsten Nachbarn charakterisiert werden. In Abb. 3.5 ist Verteilung dieser Abstände für verschiedene Regionen der Filamente bestimmt worden. Abb. 3.5 (a) zeigt die integrale Verteilung der Orte über eine Bildsequenz von 2250 Bildern. Die Blasen bewegen sich im Wesentlichen in einem begrenzten Raumbereich. Für jedes Einzelbild werden, wie in Abb. 3.5 (b) dargestellt, die Abstände zu den nächsten Nachbarn in drei verschiedenen Regionen bestimmt. Hieraus ergeben sich die Verteilungen Abb. 3.5 (c-e). Alle Verteilungen haben ein Maximum der Häufigkeit für etwa 1.5 bis $2 \mathrm{~mm}$. Die Regionen (1) und (2) befinden sich in einem Filament und werden von den Blasen nacheinander durchlaufen. Die hierzu korrespondierenden Verteilungen der Blasenabstände zeigen eine Verringerung des am häufigsten vorzufindenen Abstandes. Dies steht im Einklang mit der Beobachtung, daß Blasen-Blasen-Kollisionen in den Filamenten mit zunehmender Nähe zum Zentrum der Filamentstruktur immer wahrscheinlicher werden.

\subsubsection{Verteilung der Geschwindigkeiten}

Ein Beispiel für die Geschwindigkeitsverteilung der Blasen bei einem Druck von $p_{a}=80 \mathrm{kPa}$ und einer Frequenz $f=21.5 \mathrm{kHz}$ ist in Abb. 3.6 dargestellt. Die Verteilung zeigt, daß eine Translationsgeschwindigkeit von etwa $0.25 \mathrm{~m} / \mathrm{s}$ am häufigsten im Blasenfeld gemessen wurde. Die maximale Geschwindigkeit der Blasen beträgt in diesem Beispiel $0.85 \mathrm{~m} / \mathrm{s}$.

\subsubsection{Verteilung der Radien}

Die Messung der Blasenruheradien nach dem in Abschn. 2.6 beschriebenen Verfahren basiert auf der Messung der instantanen Radien und der anschließenden, modellbasierten Rekonstruktion der Ruheradienverteilung.

In Abb. 3.7 (a) ist die Verteilung der instantanen Blasenradien $R$ dargestellt, wie sie für verschiedene Anregungsamplituden $p_{a}=75, \ldots, 140 \mathrm{kPa}$ und bei konstanter Phasenlage bestimmt wurden. Die Verteilung enthält die in 2250 Bildern und einer Aufnahmedauer von einer Sekunde in einer Region von von $9 \times 9 \mathrm{~mm}^{2}$ gemessenen Blasenradien. Die Verteilungen sind daher in Ort und Zeit gemittelt. Sie haben für verschiedene Amplituden eine ähnliche Struktur. Die Häufigkeit nimmt für große Radien ab. Es gibt eine obere Grenze der gemessenen Radien von 90 bis $130 \mu \mathrm{m}$. Die untere Grenze der Radienverteilung ist durch die Auflösung der Meßapparatur bestimmt. Für wachsenden Druck nimmt die Häufigkeit kleiner Blasenradien deutlich zu, da nun mehr Blasen auf einen Radius größer als die Auflösungsgrenze aufschwingen und so detektiert werden können. Aus der Verteilung der gemessenen Radien können nun die Ruheradien berechnet werden. Anders als die Verteilung der gemessenen Radien zeigt die Verteilung der Ruheradien eine ausgeprägte Detailstruktur. Den maximalen und minimalen Radien der gemessenen Verteilung entspricht ein Ruheradieninterval von etwa 1 bis $40 \mu \mathrm{m}$. Für kleine Anregungsamplituden $p_{a}$ von $75 \mathrm{kPa}$ und $90 \mathrm{kPa}$ sind Ruheradien von etwa 20 bis $25 \mu \mathrm{m}$ am häufigsten vertreten. Für kleine Radien nehmen diese Verteilungen dann rasch ab. Für wachsende Anregungsamplitude steigt die Anzahl der bis zu etwa $1 \mu \mathrm{m}$ kleinen Blasen deutlich an. Eine höhere Anregungsamplitude beeinflußt aber auch die Entstehungs- und Vernichtungsprozesse in dem Blasenfeld. Daher können die den Blasenverteilungen zugrundeliegende physikalischen Prozesse einen unterschiedlichen Charakter haben. Kollisionen von Blasenpaaren lassen größere Blasen entstehen; Oberflächeninstabilitäten hingegen führen zur Zerstörung der Blasen und zum Entstehen 
(a)

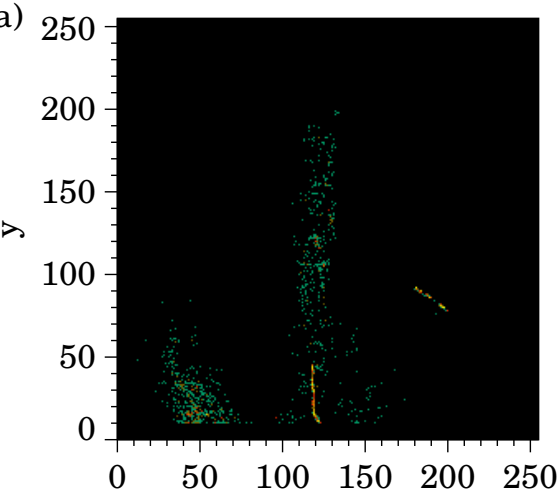

(c)

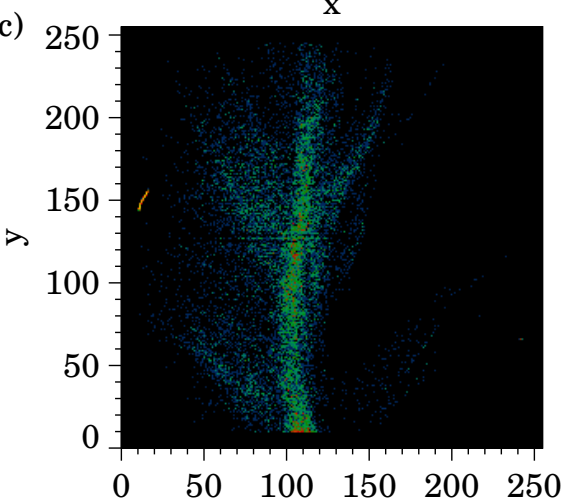

(e)
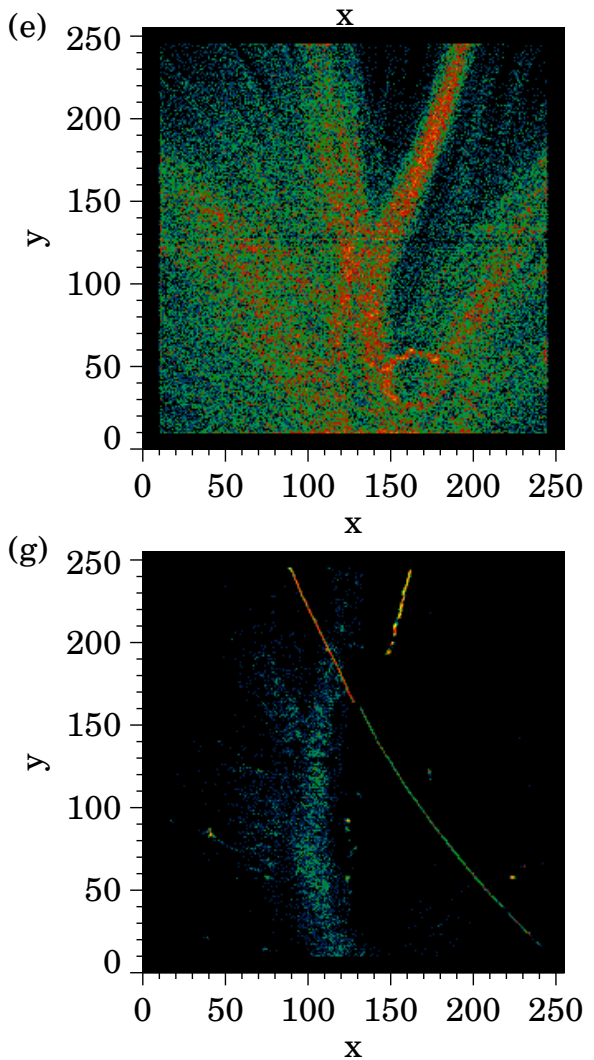
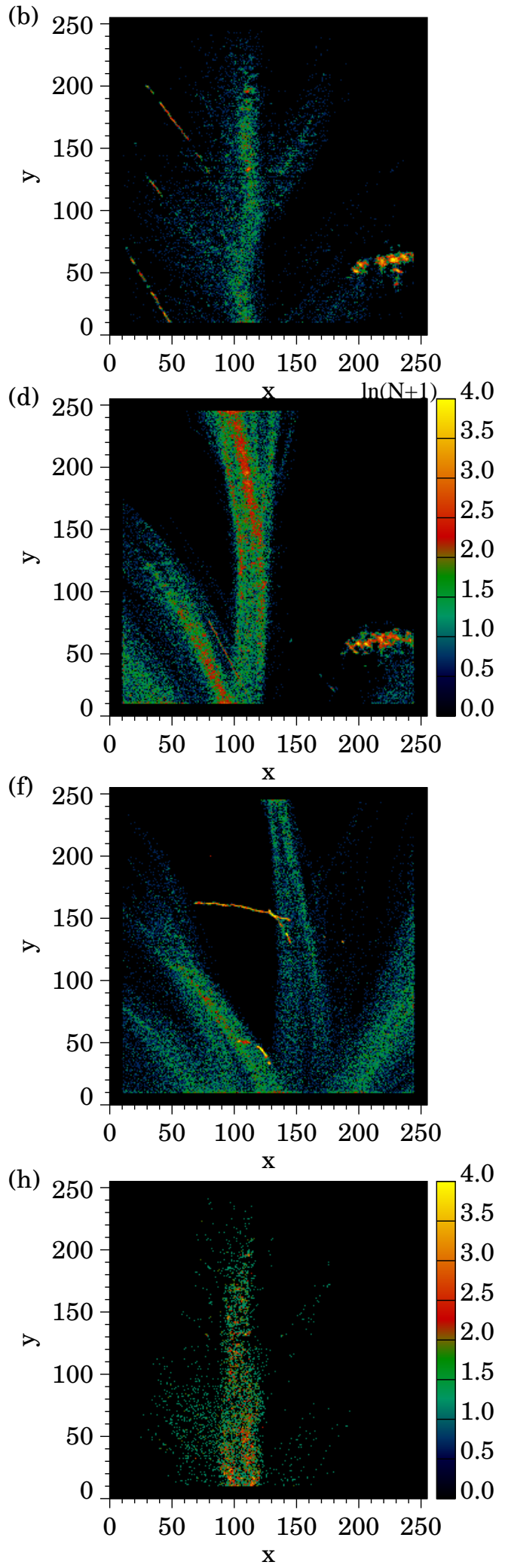

Abbildung 3.3.: Integrale Verteilung der Positionen der Blasen für verschiedene Phasen $\varphi$ der Anregung. (a-h): $\varphi=-19^{\circ}, 15^{\circ}, 58^{\circ}, 92^{\circ}, 146^{\circ}, 167^{\circ}, 213^{\circ}, 250^{\circ}$, Anregungsamplitude $p_{a}=75 \mathrm{kPa}$ und $f=21.5 \mathrm{kHz}$. 

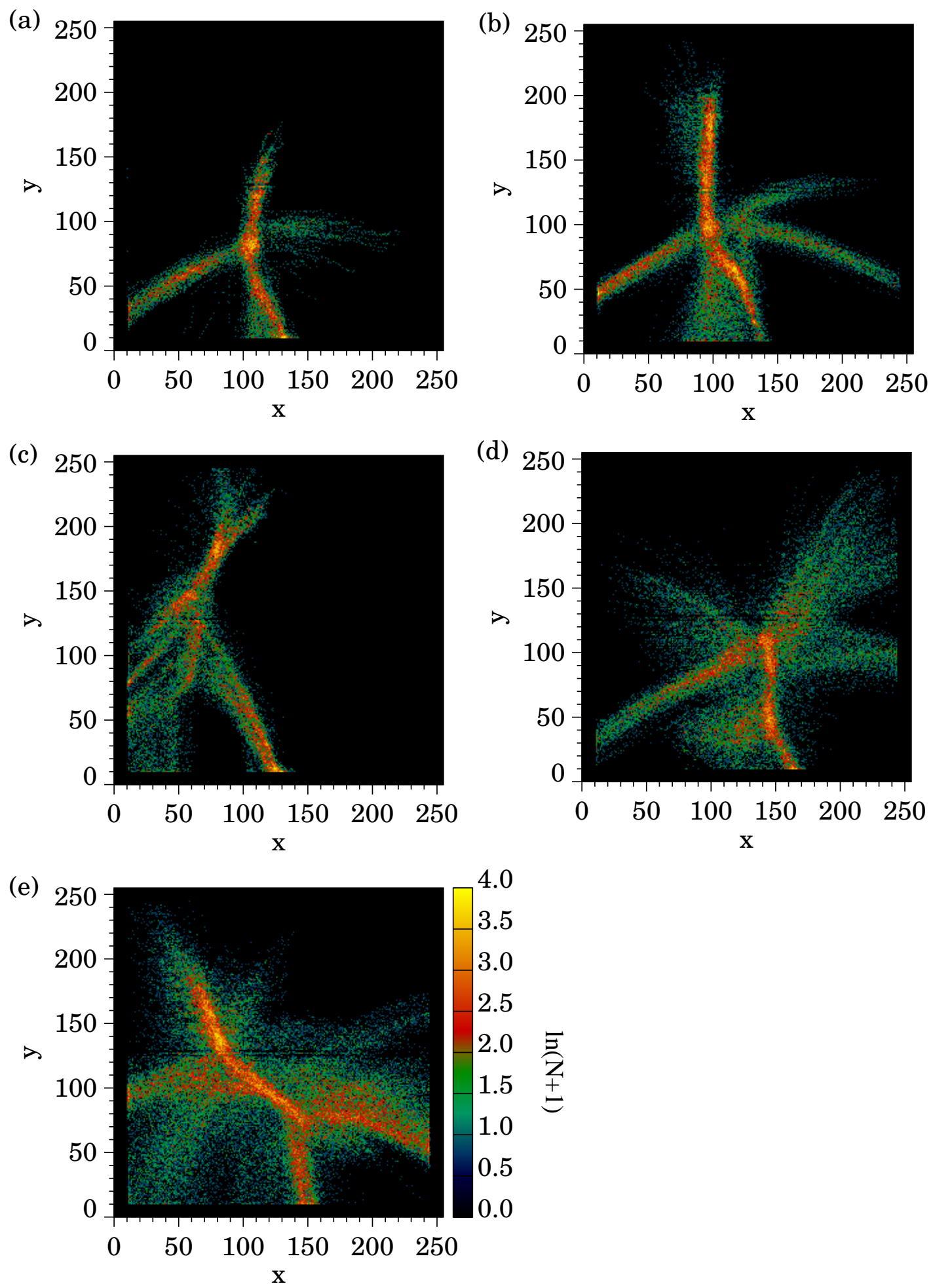

Abbildung 3.4.: Integrale Verteilung der Positionen der Blasen für $f=21.5 \mathrm{kHz}$ und verschiedene Anregungsamplituden: (a-e) $p_{a}=75,80,120,130,140 \mathrm{kPa}$; (b) Die in (e) dargestellte Farbskala ist für alle Histogramme gültig. 
(a)
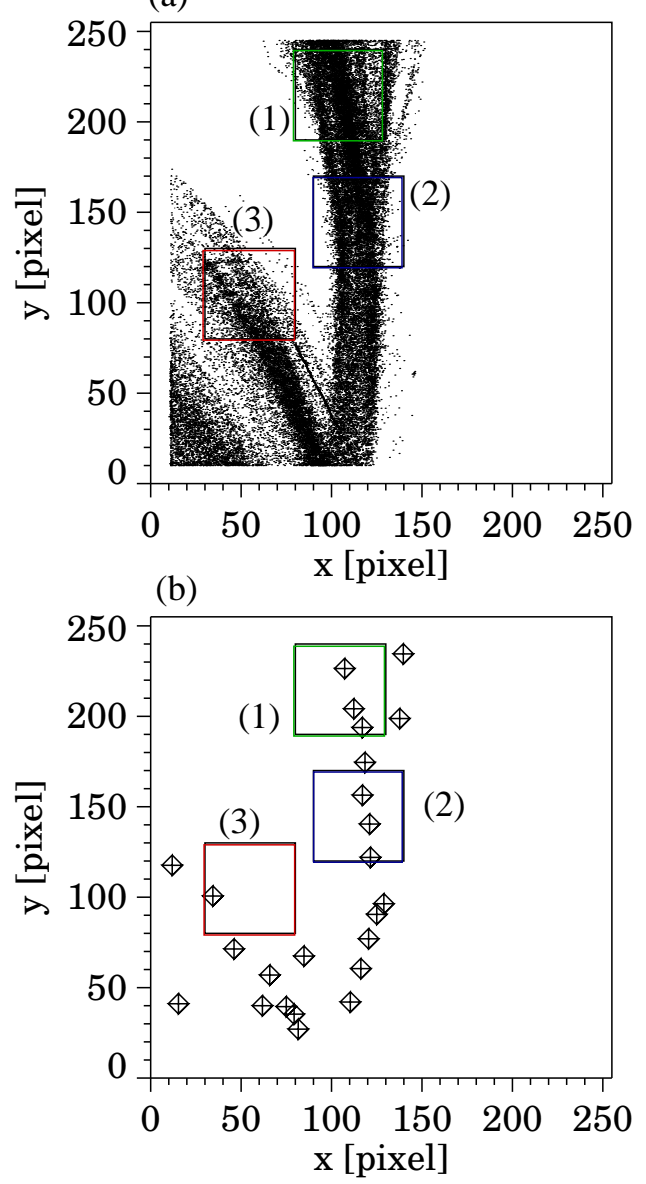

(c)
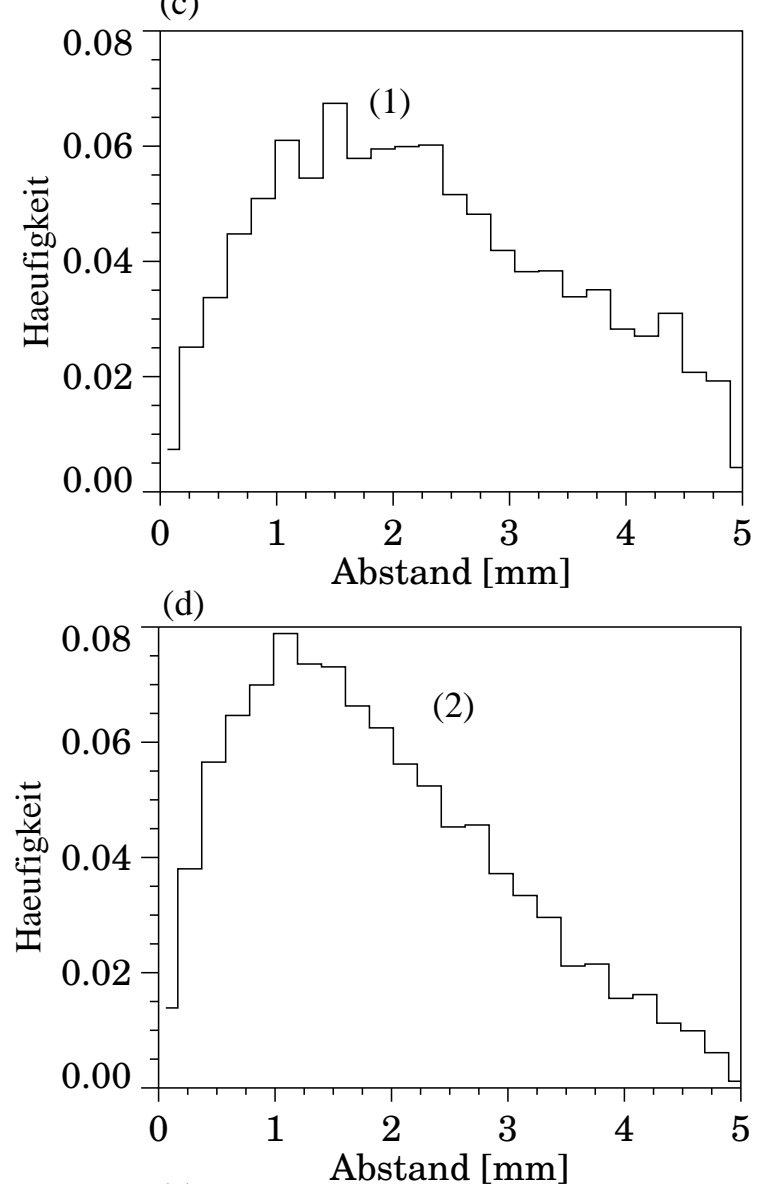

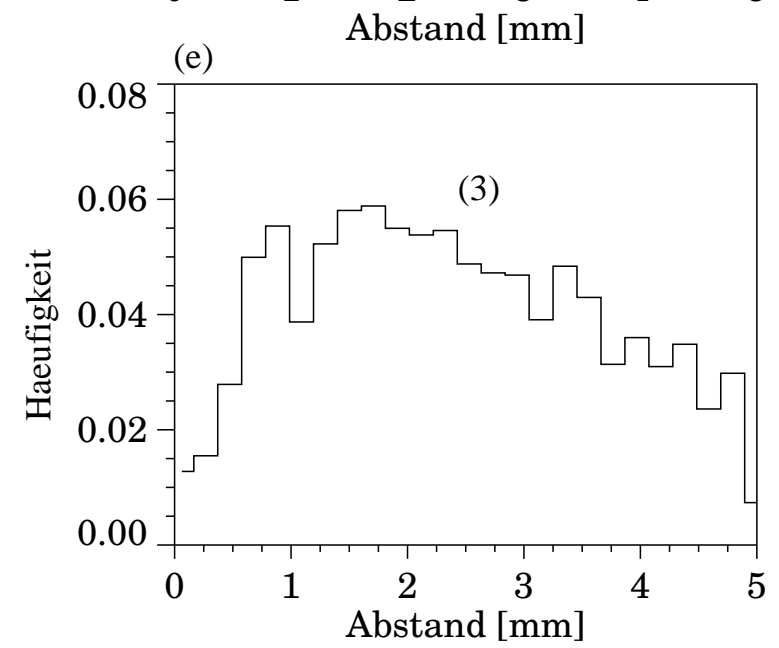

Abbildung 3.5.: Abstände nächster Nachbarn in einem Filament. (a) Verteilung der Positionen der Blasen in einem Filament, integriert über 2250 Einzelbilder. Dies entspricht einer Aufnahmedauer von einer Sekunde. Mit (1), (2) und (3) sind Regionen von $50 \times 50$ Pixeln markiert, in denen die Verteilung der Abstände zu den nächsten Nachbarn untersucht wird. (b) Ein zufällig ausgewähltes Bild aus der Sequenz. Die Abstände zu den nächsten Nachbarn werden für jedes Einzelbild in den markierten Regionen durchgeführt. (c-e) Ergebnis der Verteilung der Abstände in den Regionen (1-3). Der häufigste Abstand liegt für die gewählten Regionen bei 1.5 bis $2 \mathrm{~mm}$. 


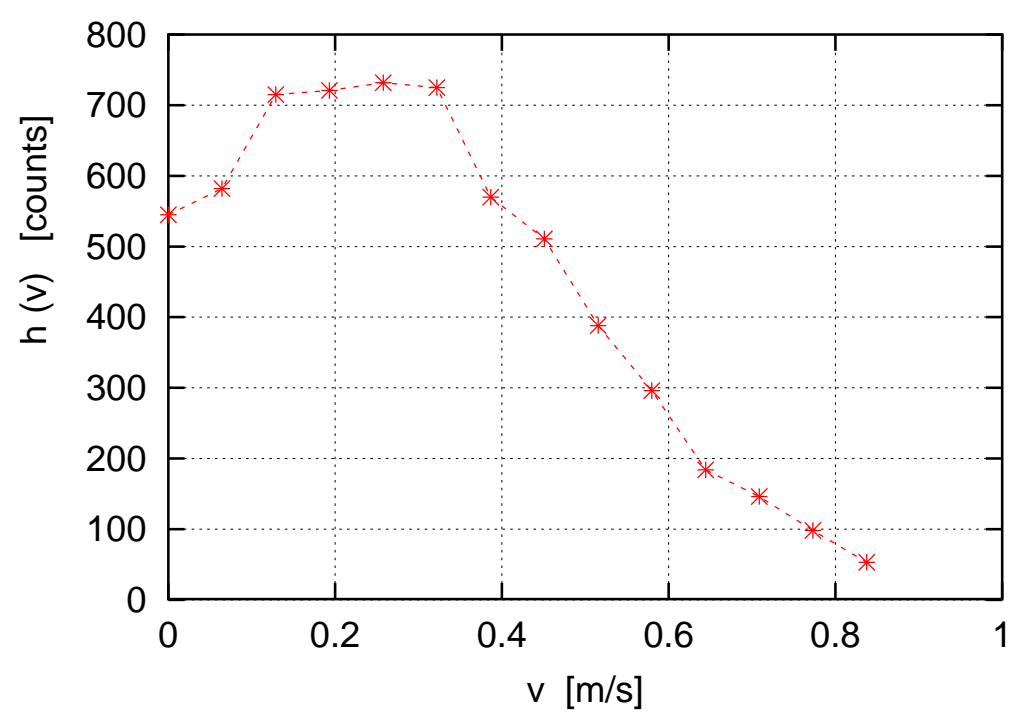

Abbildung 3.6.: Häufigkeitsverteilung der gemessenen Geschwindigkeiten für $p_{a}=80 \mathrm{kPa}$ und $f=20 \mathrm{kHz}$. Die Verteilung hat ein Maximum für etwa $0.25 \mathrm{~m} / \mathrm{s}$ und eine Maximale Geschwindigkeit von $0.85 \mathrm{~m} / \mathrm{s}$.

von kleinen Blasenfragmenten. Die Verteilung der Blasenradien zu verschiedenen Anregungsamplituden ist daher in erster Linie eine Folgen dieser komplizierten und in ihrer Wirkung oft entgegengesetzten Prozesse.

Bei konstanter Anregungsamplitude kann davon ausgegangen werden, daß die Prozesse, die zum Entstehen und zu der Zerstörung von Blasen führen, im Wesentlichen stationär sind. Für verschiedene Beobachtungsphasen werden sich, als Folge der Blasenschwingung, verschiedene Radienverteilungen einstellen.

Die im Rahmen dieser Arbeit gemessenen Radienverteilungen stimmen quantitativ und qualitativ überein mit den aus holographischen Aufnahmen akustischer Kavitationsblasenfelder gewonnenen Daten [74, 10].

\subsubsection{Trajektorien}

Aus den Positionen der Blasen können ihre Trajektorien bestimmt werden. In Abb. 3.8 (a) ist das Ergebnis einer solchen Auswertung für einige typische Trajektorien gezeigt. In dieser Darstellung befindet sich der zentrale Druckbauch unterhalb der unteren Bildkante. Die Blasen bewegen sich nahezu geradlinig in Richtung dieses Druckbauchs, bis sie in die Nähe einer anderen Trajektorie gelangen. In der Folge einer attraktiven Wechselwirkung der Blasen kommt es zu einer Kollision. Die Orte, an denen in Abb. 3.8 (a) Blasen kollidieren, sind durch Kreise gekennzeichnet. Während in den Randbereichen der Küvette Kollisionsereignisse selten sind, wird in den zentralen Raumbereichen in der Nähe des Druckbauchs die Dynamik des Kavitationsblasenfeldes durch Zusammenstöße der Blasen dominiert.

Beobachtet werden können sowohl Kollisionen von Blasen mit Blasen aus benachbarten Streamern als auch Kollisionen mit Blasen, die ihnen im selben Streamer folgen. Die Orte, an denen Kollisionen der Blasen besonders häufig beobachtet werden können, entsprechen den Punkten, an denen die Streamer zusammenstoßen. Auf diese Weise bilden sich Knotenpunkte aus, an denen sich die dendritischen Streamer verzweigen. Die Bewegung der Blasen in den Streamern 
a)

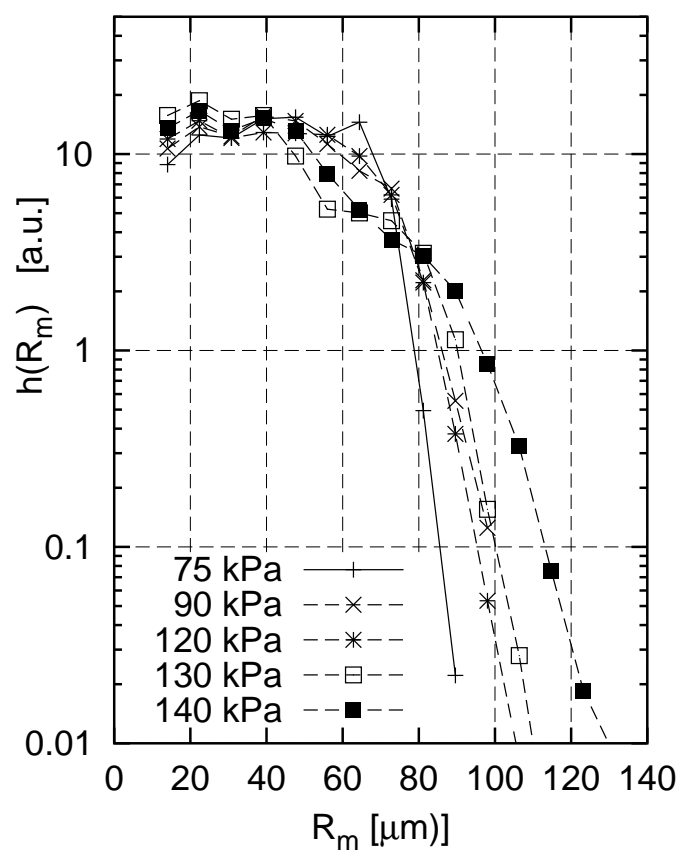

b)

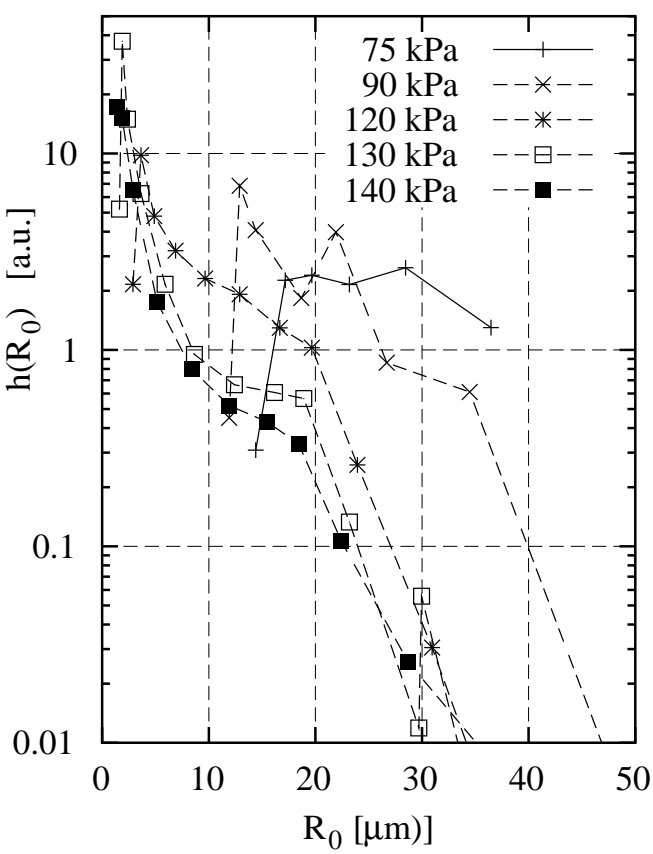

Abbildung 3.7.: Verteilung der Blasenradien für verschiedene Anregungsamplituden und konstanter Phase (Beobachtungsregion $9 \times 9 \mathrm{~mm}^{2}$ ). (a) Verteilung der gemessenen Radien $R$. (b) Verteilung der hieraus berechneten Ruheradien $R_{0}$.

nahe solchen Kollisionspunkten ist auffallend unstetig. Vereinzelt verharren Blasen, um mit nachfolgenden Blasen dort zu kollidieren und dann weiter ins Zentrum oder bis zum nächsten Kollisionspunkt zu strömen. Mitunter ändern die Blasen auch ihre ursprüngliche Bewegungsrichtung und laufen in die entgegengesetzte Richtung, um mit einer folgenden Blase zu kollidieren. Die Unstetigkeit der Translationsbewegung ist die charakteristische Eigenschaft der Blasen.

Am zentralen Druckknoten befindet sich eine in der Regel asphärische Blase, die nacheinander alle einströmenden Blasen aufnimmt. Allerdings ändert sich dabei im Laufe der Zeit ihre Größe nicht so sehr, wie es der Zuwachs an Masse durch die fortwährenden Kollisionen notwendig machen würde. Die Blase verliert offensichtlich Masse durch Fragmentierung, d.h. durch das Abspalten von Mikroblasen, die durch die Flüssigkeitsströmung aus dem Bereich des Druckknotens abtransportiert werden. Im Streulicht kann dieser Prozess als helle Halo bzw. als feiner Nebel mit bloßem Auge beobachtet werden.

Aus den Trajektorien und der Häufigkeit der Kollisionen der Blasen kann die mittlere Lebensdauer abgeschätzt werden. In den Randzonen, in denen Kollsionen unwahrscheinlich sind, kann die mittlere Lebensdauer der Blasen auf etwa 20 ms geschätzt werden. In den zentralen Bereichen, in den Kollisionen häufig auftreten, beträgt die mittlere Lebensdauer der Blasen nur etwa 2 bis $4 \mathrm{~ms}$.

\subsubsection{Blasen-Cluster}

Neben der Bewegung der Blasen in filamentartigen Strukturen wird im Experiment der Übergang dieser räumlich ausgedehnten Strukturen zu kompakten, lokalisierten Anordungen von 
(a)

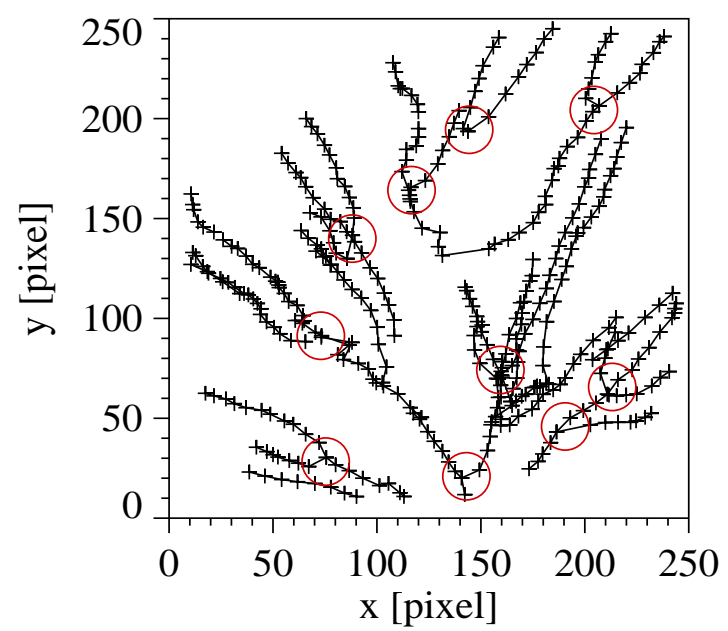

(b)

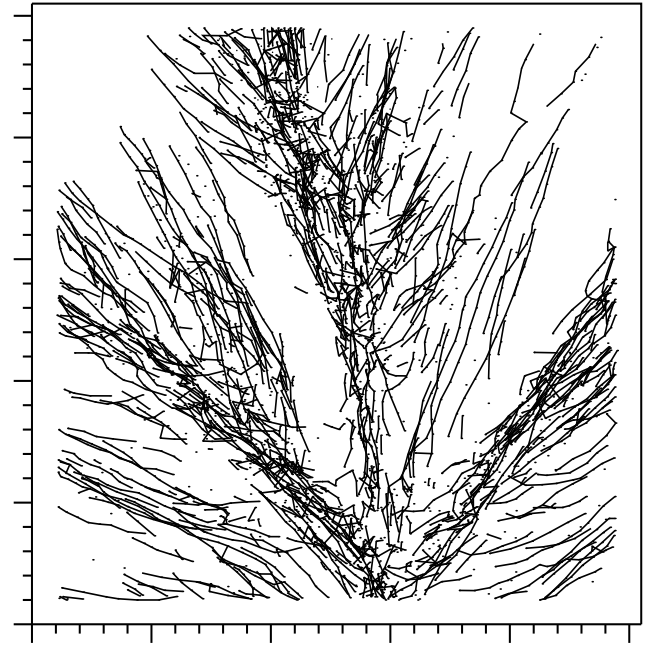

Abbildung 3.8.: Trajektorien der Blasen. (a) Ausgewählte Trajektorien der Blasen. Mit Kreisen sind die Kollisionspunkte markiert. (b) Typische Trajektorien eines Blasenfeldes, wie sie der Particle-Tracking-Algorithmus liefert.

Blasen beobachtet. Diese Objekte werden im Folgenden als Blasen-Cluster bezeichnet. Die Blasen-Cluster entstehen, wenn der Anregungsdruck $p_{a}$ den kritischen Wert überschreitet, für den die primäre Bjerknes-Kraft auf die Blasen repulsiv wird. Die Bewegung der Blasen aus den Randzonen der Küvette in den zentralen Druckbauch ist dann nicht mehr möglich. In Abb. 3.9 sind Beispiele solcher Anordnungen gezeigt, in denen sich die Blasen unmittelbar nebeneinander oder nur wenige Blasenradien voneinander entfernt gruppieren. Die Blasen in den Randbereichen des Clusters sind nahezu sphärisch. Ob im Zentrum des Clusters einzelne Blasen vorhanden sind oder aber durch Kollision größere, asphärische Objekte vorliegen, kann aus den vorliegenden Aufnahmen nicht zweifelsfrei geschlossen werden.

Die Blasen im Cluster zeigen eine starke Eigenbewegung und gruppieren den Cluster ständig neu. Eine detailierte Analyse dieser inneren und schnellen Dynamik ist jedoch mit der Zeitauflösung der im Experiment verwendeten Hochgeschwindigkeits-Video-Kamera nicht möglich und bleibt daher einer späteren Untersuchung vorbehalten. Im Vergleich zu der schnellen Dynamik im Clusterinneren ist seine Schwerpunktsbewegung mit etwa $1 \mathrm{~cm} / \mathrm{s}$ vergleichweise langsam. Ein Beispiel für die Translation der Blasencluster ist in Abb. 3.10 dargestellt. Aus der Bildsequenz ist zunächst ersichtlich, daß die Cluster während der Translation als kompakte, gebundene Objekte erhalten bleiben. Zwischen den einzelnen Clustern wirkt eine offensichtlich attraktive Wechselwirkung, die zu der Annäherung der Cluster und schließlich zu ihrer Kollision führt. Die Bewegung der Cluster in dem gezeigten Beispiel ist durch diese Cluster-ClusterKollisionen dominiert. Im Verlauf der Sequenz kollidieren die Cluster paarweise miteinander und bilden neue Cluster, bis im letzten Bild nur ein einziger Cluster übriggeblieben ist. Das Volumen der Cluster nimmt im Verlaufe der Zusammenstöße nicht in dem Maße zu, wie aus den Einzelvolumina der Stoßpartner folgen sollte. Eine in etwa konstante Größe könnte durch eine stabilitätsbedingte Abspaltung von Mikroblasen aus dem Cluster erklärt werden. In den letzten beiden Aufnahmen der Bildsequenz in Abb. 3.10 sind im Nachlauf des Clusters kleine, ihm folgende Blasen zu erkennen. 
(a)

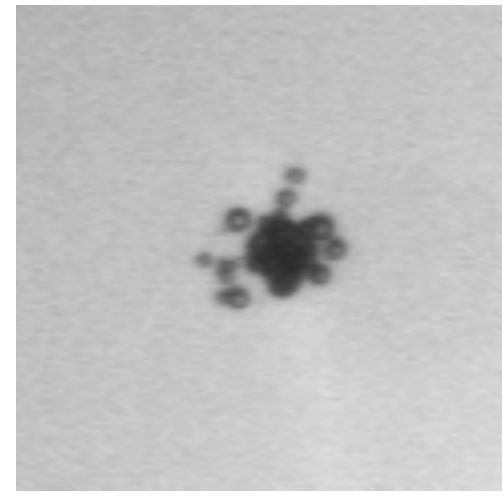

(b)

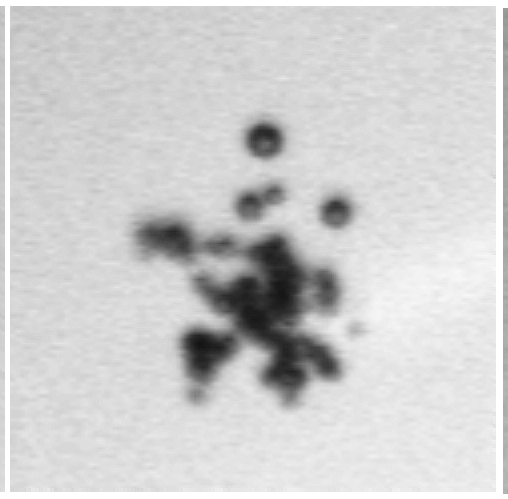

(c)

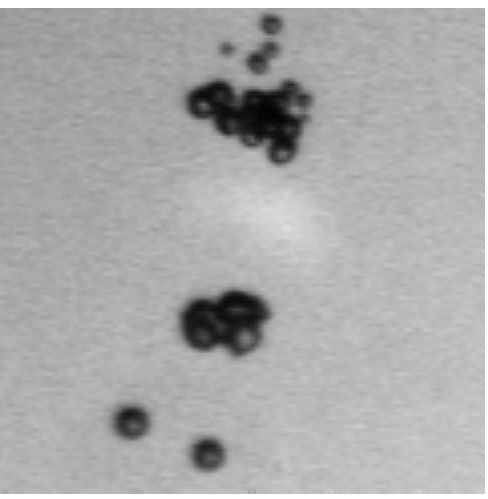

Abbildung 3.9.: (a-c) Beispiele der Anordnung der Blasen in Clustern. Die Blasen sind nahezu sphärisch. Deformationen sind sichtbar, wenn die Blasen sich unmittelbar berühren (Belichtungszeit $3 \mu \mathrm{s}$, Bildausschnitt ca. $3 \times 3 \mathrm{~mm}^{2}$ ). In (c) ist ein Beispiel für die Spaltung eines Clusters in zwei Fragmente gezeigt.

\subsection{Zusammenfassung}

In diesem Kapitel wurden die wesentlichen experimentellen Ergebnisse vorgestellt, die mit den in Kapitel 2 entwickelten und diskutieren Methoden gewonnen wurden. Die Untersuchungen wurden an Blasenfeldern, d.h. Systemen mit einer Vielzahl von Blasen, und an Systemen mit wenigen Blasen durchgeführt. Es wurden exemplarisch Messungen der Positionen und Geschwindigkeiten der Blasen gezeigt. Mit Hilfe eines Tracking-Algorithmus konnten die Trajektorien der Blasen verfolgt werden. Hierdurch war es möglich, die Kollisionen mit anderen Blasen zu verfolgen. Es wurde festgestellt, daß die Dynamik der Blasenfelder in der Nähe des Druckbauches und an den Verzweigungspunkten der dendritischen Struktur kollisionsdominiert ist. Der Übergang von der dendritischen Struktur zu kompakten, gebundenen Zuständen von Blasen konnte beoachtet und die Bewegung und Kollision dieser Objekte verfolgt werden. 

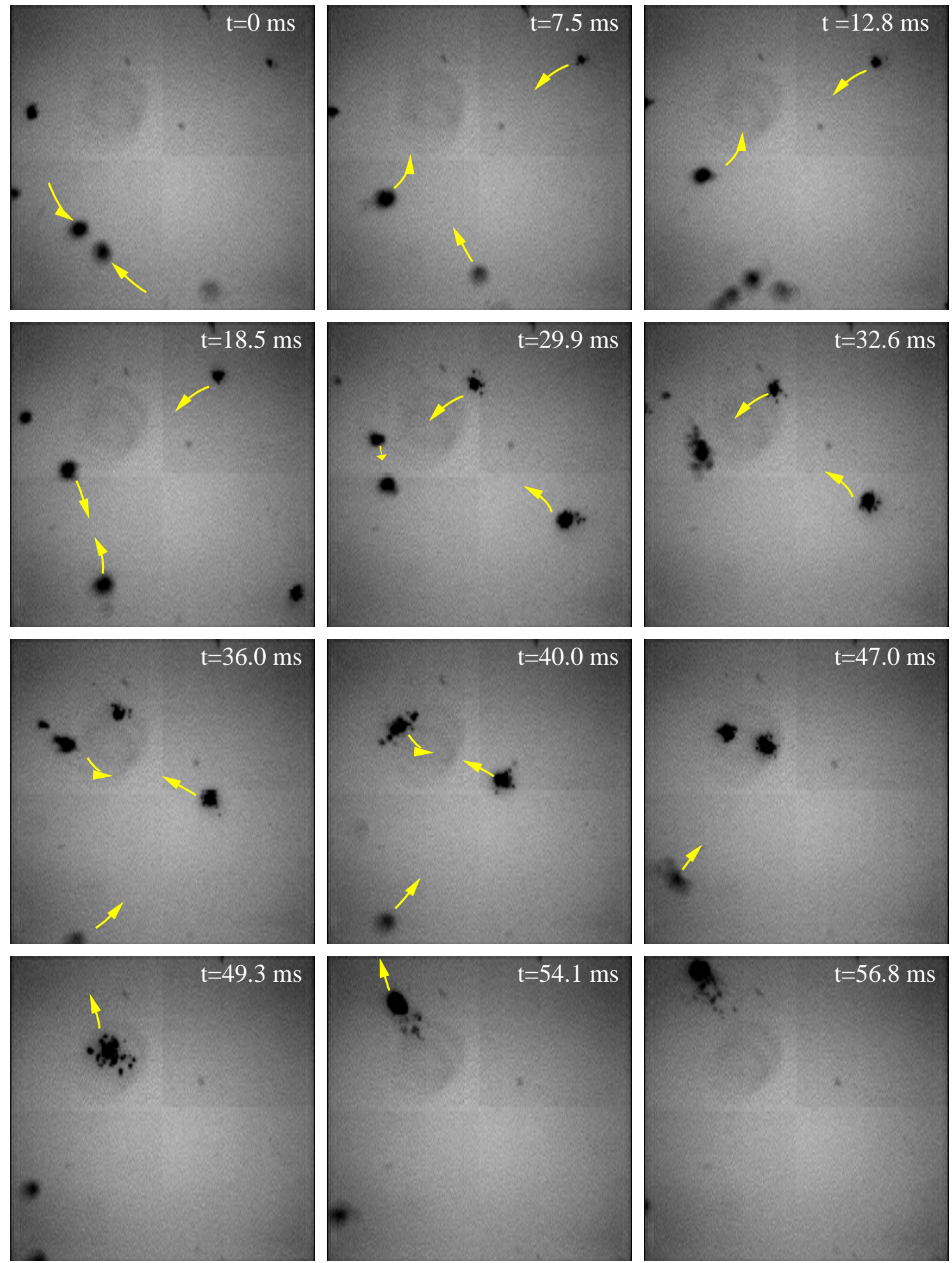

Abbildung 3.10.: Bildsequenz der Bewegung der Blasen-Cluster für einen Anregungsdruck $p_{a}=130 \mathrm{kPa}$. Der Bildausschnitt ist $9 \times 9 \mathrm{~mm}^{2}$ (Blitzdauer $\approx 3 \mu \mathrm{s}$ ). Die Zeitintervalle zwischen den dargestellten Bildern sind so gewählt, daß sie ein möglichst gutes Verständnis der Dynamik erlauben. Die momentane Bewegungsrichtung der Blasen ist durch Pfeile angedeutet. 


\section{Theorie}

In diesem Kapitel werden Modelle zur theoretischen Beschreibung akustischer Kavitationsblasenfelder vorgestellt. Grundlage jeder Modellierung sind die hydrodyamischen Grundgleichungen in Form der Erhaltungsgleichungen für Masse, Impuls und Energie. In Abschn. 4.1 werden die allgemeinene Grundlagen der Modellierung diskutiert. Die Vielfalt und Komplexität der involvierten physikalischen Prozesse macht eine Einschränkung der Modellannahmen und damit eine Abstraktion notwendig. Ausgangspunkt hierfür ist zunächst die Dimensionsanalyse der Grundgleichungen. Mit Hilfe dimensionsloser Kennzahlen werden physikalisch relevante Grenzfälle charakterisiert. Die Analyse der raum-zeitlichen Skalen des experimentellen Systems gestattet die Einteilung in verschiedene Modellklassen.

Es liegt in der Natur eines Mehrskalensystems, daß eine geschlossene theoretische Beschreibung aller involvierter Skalen ausgesprochen schwierig ist. Diese Beschreibung kann erst dann mit Aussicht auf Erfolg in Angriff genommen werden, wenn die Dynamik des Systems auf den einzelnen Skalen verstanden ist. Daher wird in dieser Arbeit zunächst das Hauptaugenmerk sowohl auf die mikroskopische als auch auf die makroskopische Beschreibung gelegt.

In den Abschn. 4.2 und 4.3 werden ein Kontinuums- und ein Partikelmodell vorgestellt. Das Kontinuumsmodell fußt auf [2,3] und wurde im Rahmen dieser Arbeit und in [51, 79] ausführlich numerisch untersucht. Das Partikelmodell beschreibt die Dynamik im Rahmen einer Lagrangeschen Formulierung inkompressibler Hydrodynamik und wurde im Rahmen dieser Arbeit entwickelt. Die beiden Modelle werden in Abschn. 4.4 kritisch verglichen.

Im Rahmen der Lagrangeschen Formulierung wird der Einfluß der Kompressibilität der Flüssigkeit vernachlässigt. In Abschn. 4.5 werden daher die Auswirkungen der Kompressibilität auf die Blasenbewegung diskutiert. Von besonderem Interesse ist hier die durch die Laufzeit des Schalls verursachte zeitverzögerte Kopplung der Blasen.

Der Abschn. 4.6 greift die grundlegende Fragestellung der Analyse der Raum- und Zeitskalen auf, die mit Hilfe der Methoden der nichtlinearen Zeitreihenanalyse durchgeführt wird. Wesentliches Ergebnis ist die Abschätzung der Dimension bzw. der Komplexität des experimentellen Systems und die daraus folgenden Implikationen für die Modellierung. Das Kapitel schließt mit einer Zusammenfassung.

\subsection{Grundlagen der Modellierung}

In diesem Abschnitt werden die Grundlagen der Modellierung eines hydrodynamischen Mehrphasensystems eingeführt und diskutiert, soweit sie für die folgende Darstellung relevant sind. Wir beginnen zunächst mit der Definition charakteristischer Kennzahlen und stellen anschließend die Ansätze der Modellierung vor. 


\subsubsection{Definition der Kennzahlen}

Grundlegend für das Verständnis hydrodynamischer Prozesse sind dimensionslose Kennzahlen. Die Reynolds-Zahl $\mathcal{R} e$ beschreibt die relative Bedeutung von Konvektion und viskoser Diffusion für den Impulstransport. In jeder Strömung von Fluiden sind beide Mechanismen vorhanden, allerdings sind sie je nach Geschwindigkeit und Geometrie der Strömung nicht von derselben Größenordnung. Die Reynolds-Zahl wird definiert durch

$$
\mathcal{R} e=\frac{\text { konvektiver Impulsfluß }}{\text { diffusiver Impulsfluß }} .
$$

Für die Bewegung einer Blase mit dem Radius $R$ und der Geschwindigkeit des Schwerpunktes $\mathbf{u}$ in einem Fluid mit der dynamischen Viskosität $\eta$ und der Dichte $\rho$ ist die Reynoldszahl bestimmt durch das Verhältnis

$$
\mathcal{R} e=\frac{2 R|\mathbf{u}|}{\nu} \quad \text { mit } \quad \nu=\eta / \rho
$$

wobei $\nu$ die kinematischen Viskosität bezeichnet. Die Gestalt einer Grenzfläche wird bestimmt durch die an ihr wirkenden Kräfte. Die Weberzahl ist definiert durch das Verhältnis von Trägheitskräften und Kräften der Oberflächenspannung

$$
\mathcal{W} e=\frac{\text { Trägheitskraft }}{\text { Kräfte der Oberflächenspannung }} .
$$

Die aus der Oberflächenspannung resultierenden Kräfte sind bestrebt, die Oberfläche der Blase kugelförmig zu halten, während die Trägheitskräfte zu einer Deformation der Grenzfläche führen. Die Weberzahl ist definiert durch

$$
\mathcal{W} e=|\mathbf{u}| \sqrt{\frac{\rho R}{\sigma}}
$$

mit der Oberflächenspannung $\sigma$. Sie ist damit ein Maß für die Sphärizität der Blase: Für $\mathcal{W} e \ll$ 1 dominieren Kräfte der Oberflächenspannung und die Blase ist sphärisch. In gleicher Weise kann die Wirkung des Auftriebs auf die Blase charakterisiert werden durch das Verhältnis der Auftriebskräfte zu den Kräften der Oberflächenspannung. Dieser Quotient wird als Eötvös-Zahl bezeichnet und ist durch

$$
\mathcal{E} e=\frac{\text { Auftriebskraft }}{\text { Kräfte der Oberflächenspannung }}
$$

definiert. Als Kennzahl für die direkte Wechselwirkung der Blasen durch Kollision wird die Knudsen-Zahl $\mathcal{K} n$ definiert. Diese wird gebildet durch das Verhältnis der mittleren freien Weglänge der Blasen und der charakteristischen makroskopischen Länge, z.B. der Länge des Resonators oder der Wellenlänge der Schallwelle:

$$
\mathcal{K} n=\frac{\text { mittlere freie Weglänge }}{\text { makroskopische Länge }} .
$$

Im Fall $\mathcal{K} n \ll 1$ ist das System kollisionsfrei, für $\mathcal{K} n \gg 1$ dominieren hingegen Stoßereignisse die Dynamik. Der Grenzfall $\mathcal{K} n \rightarrow \infty$ wird als hydrodynamischer Grenzfall bezeichnet. Auf der Grundlage der experimentellen Daten und der Raum- und Zeitskalen charakterisieren die genannten Kennzahlen die Dynamik des Systems. 


\subsubsection{Modellierungsansätze}

Eine kontinuumstheoretische Beschreibung eines heterogenen hydrodynamichen Systems mit flüssiger und gasförmiger Phase betrachtet das Gemisch als ein Kontinuum mit mittleren makroskopischen Eigenschaften. Diese effektiven Eigenschaften des Mediums werden bestimmt durch eine räumliche, zeitliche oder raum-zeitliche Mittelung. Um die makroskopischen, effektiven Eigenschaften bestimmen zu können, ist jedoch die Kenntnis der mikroskopischen Wechselwirkung der Phasen notwendig. Eine statistische Beschreibung heterogener Medien wurde von Batchelor [5] gegeben. Für geringe Blasenkonzentrationen $\alpha \approx 1 \%$ können die Wechselwirkungen der Blasen in Blasenströmungen vernachlässigt werden. Die effektiven Eigenschaften des Mediums sind dann proportional zu $\alpha$ [9]. Für höhere Blasenkonzentrationen müssen hingegen die Wechselwirkungen der Blasen berücksichtigt werden. Um die effektiven Eigenschaften des Mediums in der Ordnung $\mathcal{O}\left(\alpha^{2}\right)$ beschreiben zu können, müssen Paarwechselwirkungen betrachtet werden.

Russo et al. [69, 70] entwickeln eine kinetische Theorie für eine inkompressible Blasenströmung auf der Grundlage der Hamiltonschen Beschreibung der inkompressiblen Hydrodynamik. Ausgehend von der Liouville-Gleichung für die N-Teilchen-Verteilungsfunktion wird eine VlasovGleichung abgeleitet. Im hydrodynamischen Grenzfall werden Blasenkollisionen berücksichtigt.

Eine Beschreibung von Blasenfeldern mit Hilfe der statistischen Mechanik wird von Yurkovetsky et al. [86] gegeben.

Wie kann nun die Modellierung eines Mehrphasenystems durchgeführt werden? Wir betrachten das Kontinuumsmodell und die Partikelsimulation als diametral hinsichtlich der gewählten Skala. Ein Kontinuumsansatz beschreibt makroskopische, gemittelte Größen. Diese raum-zeitliche Mittelung führt zu dem Verlust von Information. Ein zusätzliches Problem besteht darin, daß die Gleichungen durch einen empirischen Zusatz geschlossen werden müssen. Die Direkte Numerische Simulation (DNS) löst die Navier-Stokes-Gleichungen und beschreibt so die (im hydrodynamischen Sinne) mikroskopische Dynamik. Eine weitere Möglichkeit der Simulation besteht in der Formulierung eines Partikelmodelles im Rahmen einer Hamiltionschen Formulierung der inkompressiblen Hydrodynamik.

Das Kontinuumsmodell besteht aus einem System gekoppelter partieller Differentialgleichungen für die langsam veränderliche Schallfeldamplitude, die Blasendichte und die Geschwindigkeit der Blasen. Wesentliches Ergebnis dieses Modells ist, daß eine homogene Blasenverteilung instabil ist und zu einer Selbstkonzentration der Blasen und Selbstfokussierung der Schallwelle führen kann. Zur Modellierung akustischer Blasenfelder wurden Kontinuumsmodelle vorgeschlagen [2, 3], die ihr Hauptaugenmerk auf die Eigenschaften gemittelter, makroskopischer Größen legen.

Daher wird in der vorliegenden Arbeit mit dem Partikelmodell der Ansatz der Hamiltonschen bzw. Lagrangeschen Formulierung der inkompressiblen Hydrodynamik [43, 40] verfolgt. Betont werden muß jedoch, daß die direkte Wechselwirkung der Blasen durch Kollision oder Fragmentierung auch im Rahmen dieser Theorie nicht beschrieben werden kann; dies bleibt der direkten numerischen Simulation (DNS) vorbehalten [14, 23, 32, 71]. Der Kern der Fragestellung ist daher das Verständnis der elementaren Dynamik von Translation und Oszillation einer Einzelblase auf mikroskopischer Skala und die Bedeutung der Blasenwechselwirkung für das Entstehen und die Stabilität von Strukturen auf makroskopischen Raum- und Zeitskalen. Während das Kontinuumsmodell die mittleren Eigenschaften makroskopischer Größen beschreibt, wird im Partikelmodell in Abschn. 4.3 die Lagrangesche Formulierung inkompressibler Hydrodynamik verwendet. Dieses Modell beschreibt die Kopplung von Translation und Oszillation einer Einzelblase und ihre Wechselwirkung mit anderen Blasen im Rahmen einer Multipolentwicklung. 


\subsection{Kontinuumsmodell}

Das Kontinuumsmodell beschreibt die Wechselwirkung des Schallfeldes mit einer Blasenverteilung. Es besteht aus drei gekoppelten partiellen Differentialgleichungen für die Schallfeldamplitude, die Blasengeschwindigkeit und die Blasendichte [3]. Eine lineare Stabilitätsanalyse dieses Modellgleichungssystems führt zu dem Ergebnis, daß eine homogene, monodisperse Verteilung von Blasen in einem Schallfeld instabil ist.

\subsubsection{Wellengleichung für Blasen-Flüssigkeits-Gemische}

Ein Blasen-Flüssigkeits-Gemisch kann als ein Kontinuum mit mittleren makroskopischen Eigenschaften beschrieben werden, wenn die Blasengrößen und mittleren Blasenabstände klein sind im Vergleich zu den Längen, auf denen sich die makroskopischen Eigenschaften des Gemisches signifikant ändern. Eine solche Länge ist beispielsweise gegeben durch die Wellenlänge $\lambda=2 \pi c / \omega$ eines Schallfeldes oder die geometrische Abmessung des Resonators. Auf diesen Annahmen basieren u.a. die heuristische Ableitung der Bewegungsgleichungen für das BlasenFlüssigkeits-Gemisch [80] und die mathematisch rigorose Formulierung [15]. Die dort abgeleitete Bewegungsgleichung ist gültig für ein schwach kompressibles Fluid mit geringer Blasenkonzentration $\alpha$ und führt zu der Wellengleichung für den Druck $p$ im Gemisch [18]:

$$
\frac{1}{c^{2}} \frac{\partial^{2} p}{\partial t^{2}}-\Delta p=4 \pi \rho \int_{0}^{\infty} \hat{R}_{0}^{2} N\left(\mathbf{x}, \hat{R}_{0}\right) \ddot{R} \mathrm{~d} \hat{R}_{0},
$$

wobei die Dichte $\rho$ der Flüssigkeit und die Schallgeschwindigkeit $c$ als konstant angenommen werden. $N\left(\mathbf{x}, \hat{R}_{0}\right)$ bezeichnet die Wahrscheinlichkeitsdichte, eine Blasen mit Ruheradius $\hat{R}_{0}$ am Ort $\mathbf{x}$ zu finden. Für ein monodisperses Gemisch mit Blasenruheradien $R_{0}$ erhält man mit $N\left(\mathbf{x}, \hat{R}_{0}\right)=N_{0} n(\mathbf{x}) \delta\left(\hat{R}_{0}-R_{0}\right)$

$$
\frac{1}{c^{2}} \frac{\partial^{2} p}{\partial t^{2}}-\Delta p=4 \pi \rho N_{0} n(\mathbf{x}) R_{0}^{2} \ddot{R}=\omega_{0}^{2} \rho R_{0} \epsilon n \ddot{R},
$$

mit

$$
\epsilon=\frac{c^{2} \rho}{\kappa p_{0}} \frac{4 \pi}{3} R_{0}^{3} N_{0} \quad \text { und } \quad \omega_{0}^{2}=\frac{3 \kappa p_{0}}{\rho R_{0}^{2}} .
$$

Es bezeichnen hier $N_{0}$ eine charakteristische Konzentration, $n=n(\mathbf{x})$ die normierte, dimensionslose räumliche Verteilung der Blasen, $\epsilon$ der Entwicklungsparameter und $\omega_{0}$ die lineare Resonanzfrequenz der Blasen, sofern die Oberflächenspannung vernachlässigt wird.

\subsubsection{Schallfeldamplitude}

Bei der Beschreibung der raum-zeitlichen Dynamik von Blasen-Flüssigkeits-Gemischen muß i.a. die Relativbewegung der Phasen berücksichtigt werden. Allerdings ist der Prozess der Relaxation der Blasenverteilung langsam und findet auf einer Zeitskala statt, die groß ist gegenüber der Periode der akustischen Anregung. Dies wird bestätigt durch die experimentellen Beobachtungen. Für lineare Anregungsamplituden kann daher die Wellengleichung (4.8) zur Beschreibung langsam veränderlicher Blasendichten $n(\mathbf{x})$ verwendet werden. In Abwesenheit der Blasen $\left(N_{0}=0\right)$ ist eine ebene Schallwelle, die sich entlang der z-Achse ausbreitet, eine exakte Lösung der Wellengleichung (4.8):

$$
p=p_{0}+\frac{1}{2}\left\{W_{0} \exp \left[i \omega\left(t-\frac{z}{c}\right)\right]+c . c .\right\},
$$


wobei $W_{0}$ die konstante komplexe Amplitude und c.c. die komplexe Konjugation bezeichnet. Betrachten wir kleine Störungen von $W_{0}$ senkrecht zur Ausbreitungsrichtung. Um die langsame Redistribution der Blasen zu berücksichtigen, approximieren wir die Lösung der Gl. (4.8) in der Form

$$
p=p_{0}+\frac{1}{2}\left\{W(\epsilon t, \sqrt{\epsilon} x, \sqrt{\epsilon} y) \exp \left[i \omega\left(t-\frac{z}{c}\right)\right]+c . c .\right\},
$$

mit dem in Gl. (4.9) definierten Entwicklungskoeffizient $\epsilon$ der Mehrskalenanalyse. Für typische Parameterwerte ${ }^{1}$ gilt $\epsilon \ll 1$. Um einen analytischen Ausdruck für die rechte Seite der Wellengleichung (4.8) zu finden, betrachten wir lineare Blasenoszillationen, die durch die lineare Blasengleichung beschrieben werden können. Da die Amplitude $W$ eine langsam in Raum und Zeit variierende Größe ist, kann die Lösung approximiert werden durch

$$
R^{\prime}=-\frac{1}{2} \frac{1}{\rho R_{0}\left(\omega_{0}^{2}-\omega^{2}\right)}\left\{W(\epsilon t, \sqrt{\epsilon} x, \sqrt{\epsilon} y) \exp \left[i \omega\left(t-\frac{z}{c}\right)\right]+c . c .\right\},
$$

wobei Dämpfungterme vernachlässigt wurden $(\alpha=0)$. Das Einsetzen der Gln. (4.11) und (4.12) in Gl. (4.8) und das Vernachlässigen von Termen $O\left(\epsilon^{2}\right)$ führt - im Grenzfall kleiner Frequenzen ${ }^{2}$ $\omega \ll \omega_{0}$ - zu einer partiellen Differentialgleichung für die komplexe Amplitude der Wellengleichung:

$$
i \frac{\partial w}{\partial \xi}=\frac{\partial^{2} w}{\partial \eta^{2}}+\frac{\partial^{2} w}{\partial \zeta^{2}}+n w
$$

Die dimensionslosen Variablen $\xi, \eta, \zeta$ und $w$ sind definiert durch

$$
\xi=\frac{1}{2} \omega \epsilon t, \quad \eta=\frac{\omega}{c} \sqrt{\epsilon} x, \quad \zeta=\frac{\omega}{c} \sqrt{\epsilon} y, \quad w=\frac{W}{W_{0}} .
$$

Die Gl. (4.13) ist eine Amplitudengleichung vom Typ der nichtlinearen Schrödinger-Gleichung, deren Potential durch die Blasendichte $n$ gegeben ist. Die nichtlineare Schrödinger-Gleichung beschreibt die Ausbreitung von solitären Wellen und Solitonen in bestimmten nichtlineare Medien. Sie ist ein Beispiel für dispersive Strukturbildung (siehe [51, 79] für weitere Referenzen).

\subsubsection{Blasengeschwindigkeit}

Auf alle Blasen mit einem Volumen $V$ wirkt die primäre Bjerknes-Kraft, für die mit Gl. (4.11) und (4.12) der Ausdruck

$$
\mathbf{F}_{B}=\gamma_{1}\left(\frac{\partial\left(|W|^{2}\right)}{\partial \eta}, \frac{\partial\left(|W|^{2}\right)}{\partial \zeta}, 0\right), \quad \gamma_{1}=\frac{3 V_{0}}{4 \rho R_{0}^{2}\left(\omega_{0}^{2}-\omega^{2}\right)} \frac{\omega}{c} \sqrt{\epsilon}
$$

abgeleitet werden kann [3]. Die Wechselwirkung der Blasen mit der Flüssigkeit wird durch die Stokessche Reibung $\mathbf{F}_{S}$ und die Trägheitskraft ${ }^{3} \mathbf{F}_{M}$ in einfachster Form berïcksichtigt:

$$
\begin{aligned}
\mathbf{F}_{S} & =-6 \pi \mu_{l} R_{0} \mathbf{U} \\
\mathbf{F}_{M} & =-\frac{1}{2} \rho V_{0} \frac{\partial \mathbf{U}}{\partial t} .
\end{aligned}
$$

\footnotetext{
${ }^{1} \sigma=0.0725 \mathrm{Nm}^{-1}, \rho=998 \mathrm{~kg} / \mathrm{m}^{3}, \kappa=1.4 \eta=0.001 \mathrm{Ns} \mathrm{m}^{-2}, p_{0}=100 \mathrm{kPa}, c=1500 \mathrm{~m} \mathrm{~s}^{-1}$ und $\omega=2 \pi \cdot 20 \mathrm{kHz}$

${ }^{2}$ Dies wird als low-frequency limit bezeichnet.

${ }^{3}$ Als träge Masse wird die sog. zusätzliche Masse bzw. added mass betrachtet. Da die Blasen selbst nach Annahme masselos sind, sind virtuelle Masse und zusätzlichen Masse gleich. Für eine ausführliche Diskussion siehe Abschn. 4.3.4.
} 


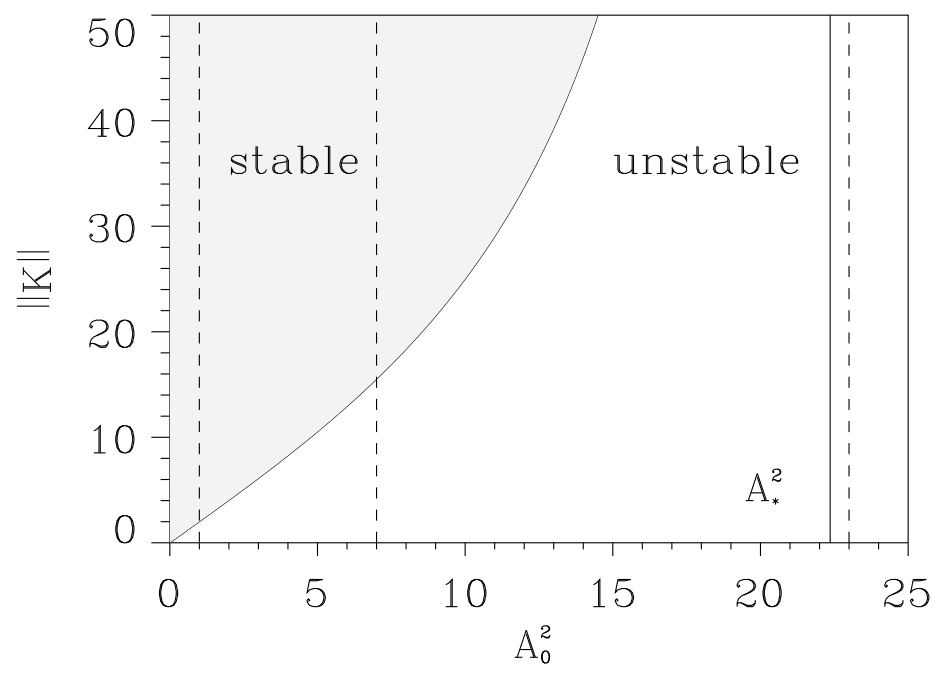

Abbildung 4.1.: Stabilitätsdiagramm für periodische Störungen der homogenen Lösung (4.23) des Kontuummodells $\left(\tau_{1}=1, \tau_{2}=0.1, \gamma=0.001, A_{\infty} \rightarrow \infty\right)$. Störungen mit Amplituden $A_{0}<A_{*}$ und hinreichend großen Wellenzahlen $\|\mathbf{K}\|=$ $\left[K_{x}^{2}+K_{y}^{2}\right]^{\frac{1}{2}}$ klingen ab. Die homogene Lösung ist instabil für langwellige Störungen (d.h. $\|\mathbf{K}\|$ klein) oder für Amplituden $A_{0}$ über dem Schwellwert $A_{*}$, der durch die durchgezogene senkrechte Linie markiert ist. Die gestrichelte Linie zeigt drei Werte für $A_{0}$, die in Abb. 4.2 verwendet werden.

Es bezeichnet hierbei $\mathbf{U}$ die langsame mittlere Driftgeschwindigkeit der Blasen. Wenn die Masse des Gases in den Blasen, die sekundäre Bjerknes-Kraft und der Auftrieb vernachlässigt werden können, so folgt

$$
\mathbf{F}_{B}+\mathbf{F}_{S}+\mathbf{F}_{M}=\mathbf{0} .
$$

Die Bewegungsgleichung für die langsame Drift der Blasen hat daher die Form

$$
\tau_{2} \frac{\partial \mathbf{u}}{\partial \xi}+\mathbf{u}=\gamma \nabla_{\eta \zeta}\left(|w|^{2}\right)
$$

wobei die folgenden Größen definiert wurden:

$$
\mathbf{u}=\frac{\mathbf{U}}{U_{*}}, \quad U_{*}=\frac{c}{2} \sqrt{\epsilon}, \quad \gamma=\frac{\gamma_{1}}{6 \pi \mu_{l} R_{0}} \frac{W_{0}^{2}}{U_{*}}, \tau_{2}=\frac{\rho V_{0}}{24 \pi \mu_{l} R_{0}} \omega \epsilon .
$$

\subsubsection{Blasendichte}

Wenn die langsame Evolution der Blasendichte betrachtet werden soll, so muß berücksichtigt werden, daß sich die Blasen bei fehlendem Schallfeld nach einer charakteristischen Zeit auflösen. Daher wird eine exponentielle Abnahme der Blasendichte bei fehlendem Schallfeld angenommen. Allerdings hat ein Schallfeld auch die Erzeugung von Blasen zur Folge. Für kleine Schalldrücke ist der Energiestrom, der zur Blasenerzeugung führt, proportional zu der Intensität des Schallfeldes [61]. Hohe Intensitäten führen zu einem Sättigungsphänomen und damit zu einer maximalen Blasendichte $N_{\infty}$ infolge der begrenzten Menge gelösten Gases in der Flüssigkeit. Die genannten Effekte werden heuristisch in der Kontinuitätsgleichung für die Blasendichte 


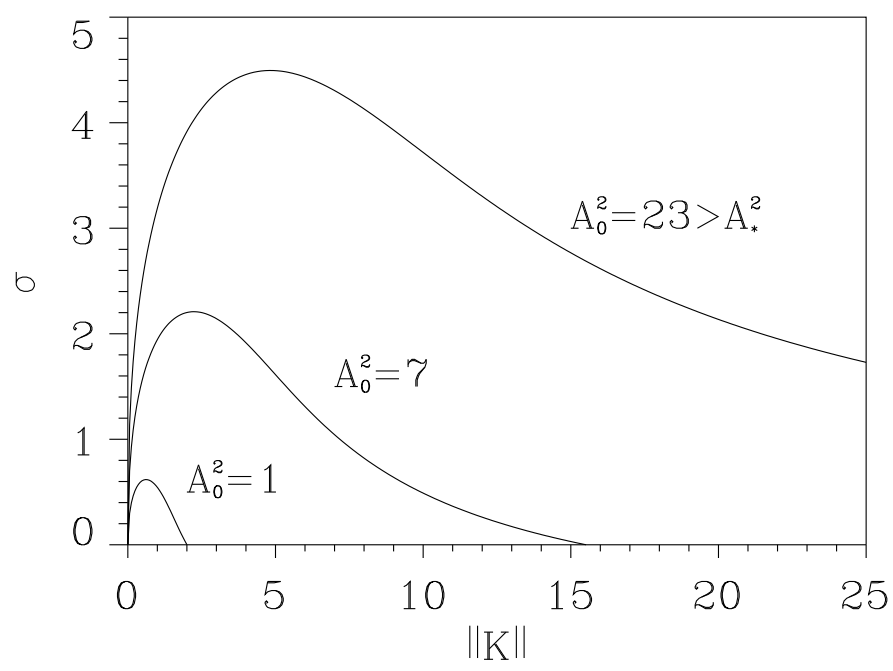

Abbildung 4.2.: Wachstumsraten $\sigma$ der (instabilen) Moden als Funktionen der Wellenzahl $\|\mathbf{K}\|$ für $A_{0}=1,7,23$ und $\tau_{1}=1, \tau_{2}=0.1, \gamma=0.001, A_{\infty} \rightarrow \infty$ (vgl. Abb. 4.1). Wenn die Wellenzahl $\|\mathbf{K}\|$ der Störung hinreichend klein ist, so können auch höhere Harmonische mit $2\|\mathbf{K}\|, 3\|\mathbf{K}\|$ usw. durch die Langwellen-Instabilität angeregt werden.

berücksichtigt:

$$
\begin{gathered}
\frac{\partial n}{\partial \xi}+\nabla_{\eta \zeta}(n \mathbf{u})=-\frac{n-f\left(|w|^{2}\right)}{\tau_{1}} \\
f\left(|w|^{2}\right)=A_{\infty}^{2}\left[1-\exp \left(-|w|^{2} / A_{\infty}^{2}\right)\right] .
\end{gathered}
$$

$\tau_{1}$ ist hierbei die dimensionslose charakteristische Zeit, mit der sich Blasen auflösen, und die Funktion $f\left(|w|^{2}\right)$ beschreibt die Sättigung der Blasenerzeugung durch das Schallfeld mit

$$
\lim _{|w| \rightarrow \infty} f\left(|w|^{2}\right)=A_{\infty}^{2}=N_{\infty} / N_{0}
$$

\subsubsection{Lineare Stabilitätsanalyse}

Eine analytische Lösung des Kontinuumsmodells (4.13), (4.19) und (4.21) ist gegeben durch

$$
A=A_{0}=\text { const. }, \quad n=f\left(A_{0}^{2}\right), \quad \Theta=-f\left(A_{0}^{2}\right) \xi, \quad u_{x}=0, \quad u_{y}=0,
$$

wobei die Amplitude $A$ und die Phase $\Theta$ definiert sind durch

$$
w=A(\xi, \eta, \zeta) \exp (i \Theta(\xi, \eta, \zeta)) .
$$

Die Gln. (4.23) und (4.24) beschreiben eine monochromatische ebene Welle, die sich in einem Flüssigkeits-Blasen-Gemisch mit homogener und stationärer Blasenverteilung ausbreitet. Die Entwicklung kleiner periodischer Störungen dieser Lösung,

$$
\left(\begin{array}{c}
\tilde{A} \\
\tilde{\Theta} \\
\tilde{n} \\
\tilde{u}_{x} \\
\tilde{u}_{y}
\end{array}\right)=\left(\begin{array}{c}
\hat{A} \\
\hat{\Theta} \\
\hat{n} \\
\hat{u}_{x} \\
\hat{u}_{y}
\end{array}\right) \exp \left(\sigma \xi+i K_{x} \eta+i K_{y} \zeta\right)
$$



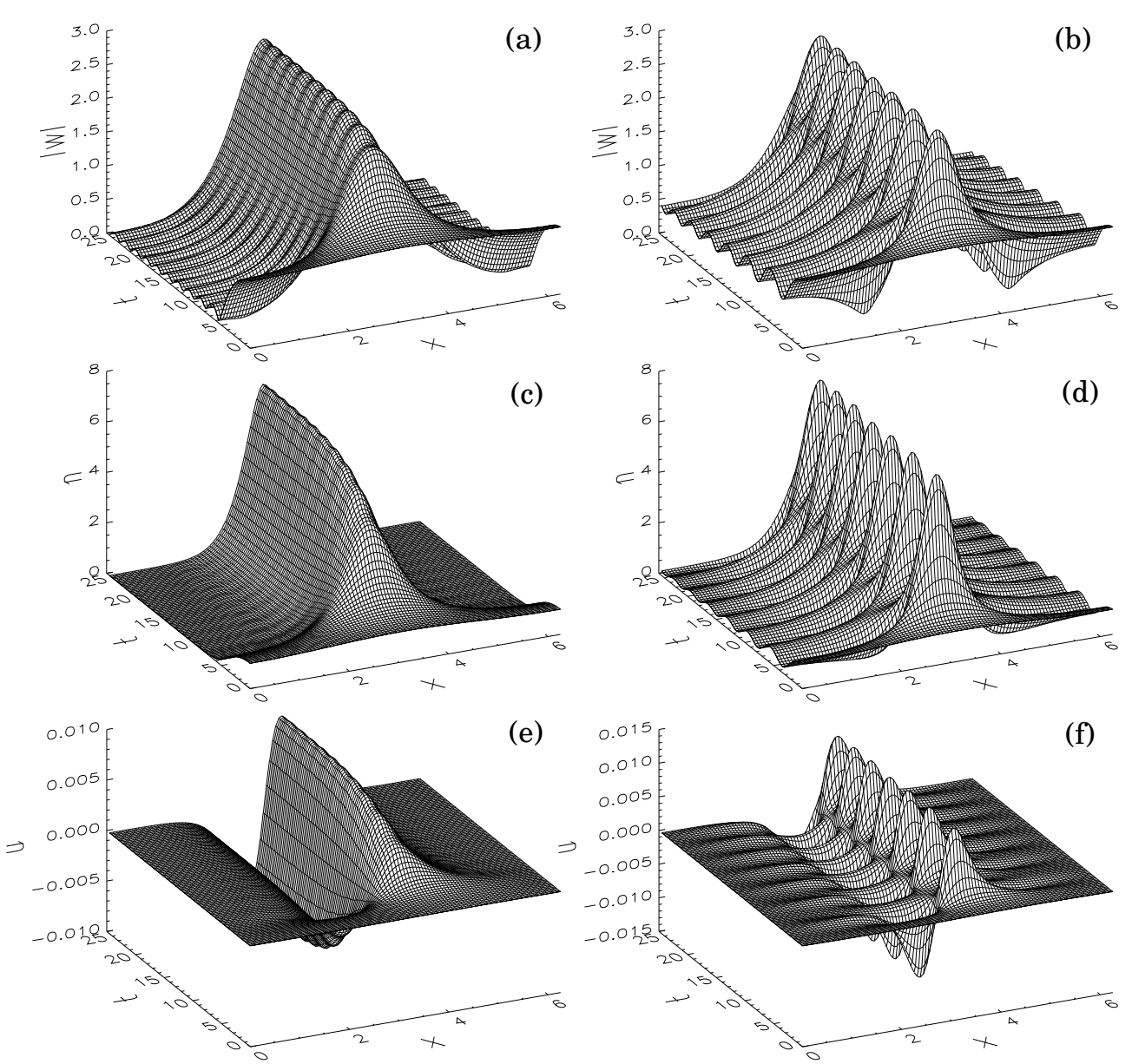

Abbildung 4.3.: Raum-zeitliche Entwicklung des Betrages (a),(b) der Schallfeldamplitude $|w|$, (c), (d) der Blasendichte $n$ und (e), (f) der Blasengeschwindigkeit $u$ für die Parameter $\tau_{1}=1, \tau_{2}=0.1, \gamma=0.001, A_{\infty} \rightarrow \infty, K=1$ ((a),(c),(e)) und $\tau_{1}=0.01, \tau_{2}=0.01, \gamma=0.001, A_{\infty} \rightarrow \infty, K=1((\mathrm{~b}),(\mathrm{d}),(\mathrm{f}))$.

wird durch die Linearisierung der Gl. (4.13), (4.19), und (4.21) beschrieben. Die Stabilität dieser Lösung wird bestimmt durch das Vorzeichen des Realteils der Wachstumsrate $\sigma$. Dieser ist positiv für langwellige Störungen, wie aus der Abb. 4.1 ersichtlich. Für Amplituden $A_{0}$, die größer als ein kritischer Schwellwert $A_{*}$ sind, sind alle homogenen Lösungen des Systems instabil für alle Wellenzahlen $\mathbf{K}=\left(K_{x}, K_{y}\right)$. Amplituden $A_{0}$, welche kleiner als $A_{*}$ sind, führen zu einem effektiven Selektionsprozeß der Strukturbildung, da in diesem Fall Störungen mit kleinen Wellenlängen zunehmen. Die verbleibende langwellige Instabilität kann als Ursache der Strukturbildung interpretiert werden, wobei zusätzlich die unterschiedlichen Wachstumsraten instabiler Moden berücksichtigt werden müssen. In Abb. 4.2 sind diese Wachstumsraten dargestellt als Funktion von $\|\mathbf{K}\|$ für drei Werte der Amplitude $A_{0}$.

\subsubsection{Numerische Simulation}

In diesem Abschnitt werden ausgewählte numerische Lösungen der Modellgleichungen (4.13), (4.19) und (4.21) für eine eindimensionale Wellenfront diskutiert, die sich entlang der z-Achse in einem Kanal der Kantenlänge $L=2 \pi / k$ ausbreitet. Eine detaillierte numerische Untersuchung 

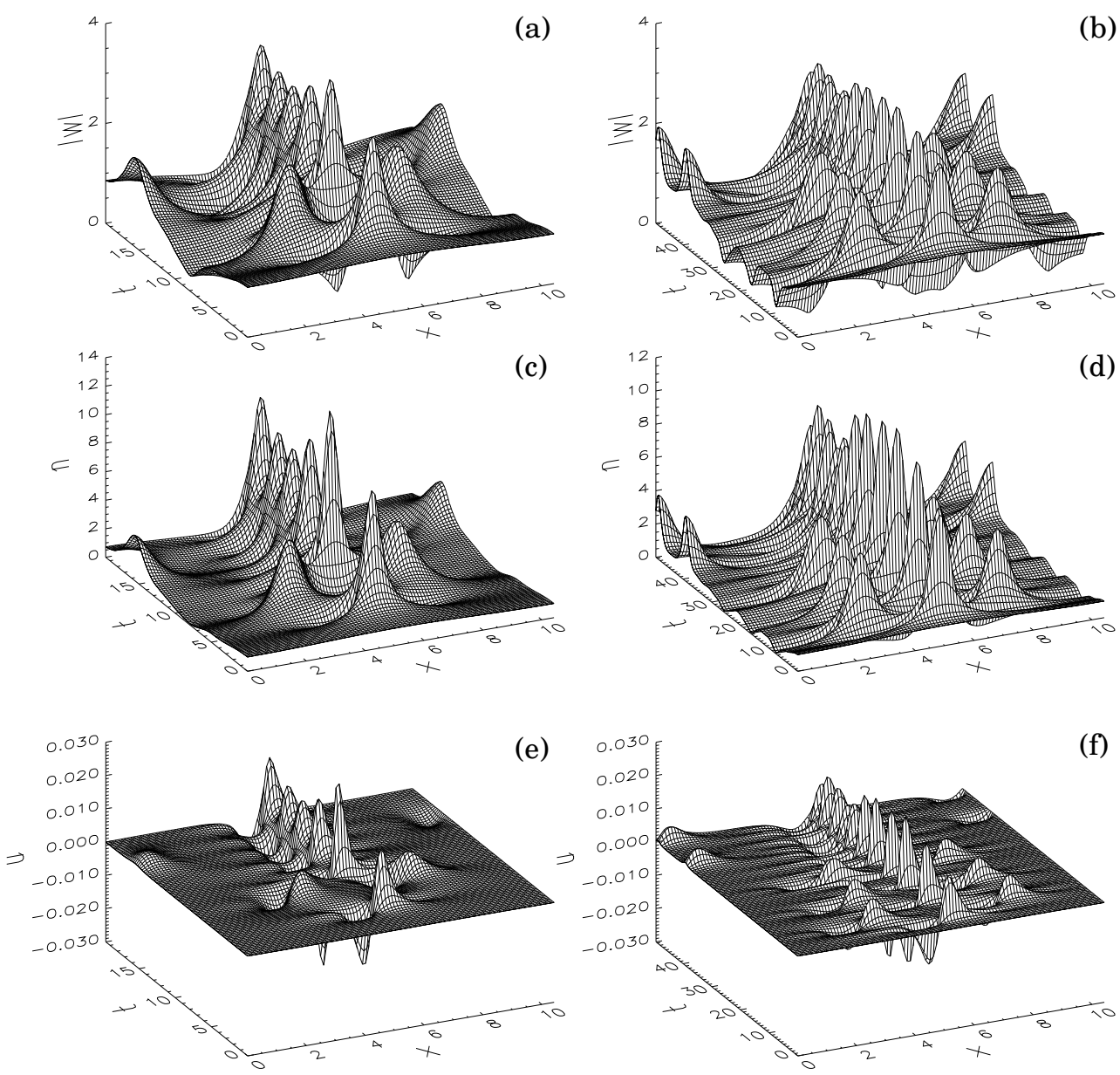

Abbildung 4.4.: Raum-zeitliche Entwicklung des Betrages (a),(b) der Schallfeldamplitude $|w|$, (c), (d) der Blasendichte $n$ und (e), (f) der Blasengeschwindigkeit $u$ für die Parameter $\tau_{1}=0.001, \tau_{2}=0.01, \gamma=0.001, A_{\infty} \rightarrow \infty, K=0.6$ ((a),(c),(e)) und $\tau_{1}=0.005, \tau_{2}=0.01, \gamma=0.001, A_{\infty} \rightarrow \infty, K=0.6((\mathrm{~b}),(\mathrm{d}),(\mathrm{f}))$.

ist in $[51,79]$ zu finden. Die Randbedingungen sind gegeben durch

$$
\begin{aligned}
\frac{\partial w}{\partial \eta}(\xi, 0) & =0=\frac{\partial w}{\partial \eta}(\xi, L) \\
u(\xi, 0) & =0=u(\xi, L) \\
n(\xi, 0) & =0=n(\xi, L)
\end{aligned}
$$

und die folgenden Anfangsbedingungen werden verwendet:

$$
\begin{aligned}
& w(0, \eta)=w_{0}\left[1+\frac{w_{1}}{2}[1-\cos (\eta)]\right], \quad \eta \in[0, L] \\
& u(0, \eta)=0 \\
& n(0, \eta)=|w(0, \eta)|^{2}
\end{aligned}
$$

mit $w_{0}=1$ und $w_{1}=0.05$. Eine typische transiente Entwicklung, die der (linearen) Langwelleninstabilität folgt, ist in Abb. 4.3 gezeigt. 
Die Instabilität führt zu einer Selbstfokussierung der akustischen Wellen und zur Selbstkonzentration der Blasendichte. Die Blasen werden in Raumbereiche höheren Schalldruckes bewegt und führen dort zu einer lokalen Reduzierung der Schallgeschwindigkeit. Daher wächst der Schalldruck dort weiter, bis nichtlineare Effekte zu einer Sättigung dieses Selbstkonzentrationsprozesses führen. Da $\tau_{1}$ die charakteristische Lebensdauer der Blasen beschreibt, hat eine Erhöhung von $\tau_{1}$ eine stärkere Dämpfung der transienten Oszillation zur Folge und einen rascheren Übergang in eine quasi-asymptotische Lösung. Durch die Nichtlinearität können instabile Moden höherer Ordnung angeregt werden. Beispiele mit zwei instabilen Moden sind in Abb. 4.4 mit nichtlinearer Kopplung der ersten $(k=0.6)$ und der zweiten Mode $(k=1.2)$ dargestellt.

Abschließend sollen die Beschränkungen des vorgestellten Kontinuumsmodells erwähnt werden. Die in den Abb. 4.3 und 4.4 dargestellten Lösungen zeigen lokal ein Anwachsen der Blasendichte. Es muß daher verifiziert werden, daß die dem Modell zugrundeliegenden Annahmen, insbesondere die der geringen Blasendichte, eingehalten werden. Auch vernachlässigt das Modell die Blasen-Blasen-Wechselwirkung. Insbesondere für hohe Blasendichten, wie sie im Verlaufe des Selbstkonzentrationsprozesses auftreten, kann diese Form der Wechselwirkung eine wesentliche Rolle spielen. Und schließlich ist das Modell per Konstruktion auf Anregungsamplituden durch das äußere Schallfeld begrenzt, für die die Blasenoszillation linear approximiert werden kann; diese Annahme ist für die experimentellen Bedingungen i.d.R. nicht erfüllt.

\subsection{Partikelmodell}

In diesem Abschnitt wird ein Modell der nichtlinearen Oszillation und Translation der Blasen in der Flüssigkeit entwickelt [50]. Es werden hierfür bewußt stark vereinfachende Annahmen in der hydrodynamischer Beschreibung der Flüssigkeit und des Gases in den Blasen gemacht. Das primäre Ziel der Modellentwicklung ist, die nichtlineare Kopplung der Translation und Oszillation der Blasen und ihre Wechselwirkung miteinander zu beschreiben. Bereits eine einzelne Blase zeigt eine außerordentlich komplexe nichtlineare Dynamik. Durch die numerische Simulation der Bewegungsgleichungen wird in diesem Abschnitt exemplarisch gezeigt, daß bereits diese einfache Beschreibung zu komplexen Lösungen führt.

Für die hydrodynamische Beschreibung des Systems werden im folgenden Annahmen über die Eigenschaften der gasfömigen und flüssigen Phase sowie der Grenzfläche gemacht. Es wird angenommen, daß die Bewegung der Flüssigkeit durch eine inkompressible, viskose Potentialströmung beschrieben werden kann. Für die Reynoldszahl gilt $\mathcal{R} e \gg 1$, d.h. der Impulsfluß in der Flüssigkeit ist konvektiv dominiert. Wir gehen davon aus, daß sich die reale Strömung um die Blase nur in einer dünnen Grenzschicht in der Nähe der Oberfläche und in einem relativ schmalen Bereich des Nachlaufs hinter der Blase von einer Potentialströmung unterscheidet. Für die Bewegung der Blasen für moderate Reynolds-Zahlen ist das hervorgerufene Strömungsfeld beinahe vollständig wirbelfrei. Die Dicke der Grenzschicht ist von der Ordnung $\mathcal{O}\left(\mathcal{R} e^{-1 / 2}\right)$ und der Durchmesser der Nachlaufströmung ist $\mathcal{O}\left(\mathcal{R} e^{-1 / 4}\right)[59,60,73]$.

Für die an der Grenzfläche wirkenden Kräfte wird angenommen, daß die Kräfte der Oberflächenspannung gegenüber Trägheitskräften überwiegen und daher die Blasen sphärisch sind. Für die dimensionslosen Weber- und Eötvös-Zahlen gilt also $\mathcal{W} e \ll 1$ und $\mathcal{E} e \ll 1$. Auftriebskräfte können i.a. vernachlässigt werden.

Zur Vereinfachung der Beschreibung wird ferner angenommen, daß die Phasengrenze nicht permeabel ist, daß mit anderen Worten kein Massen- oder Energieaustausch durch die Grenzfläche stattfindet. Ferner ist die Flüssigkeit frei von oberflächenaktiven Substanzen. Für das Verhältnis 


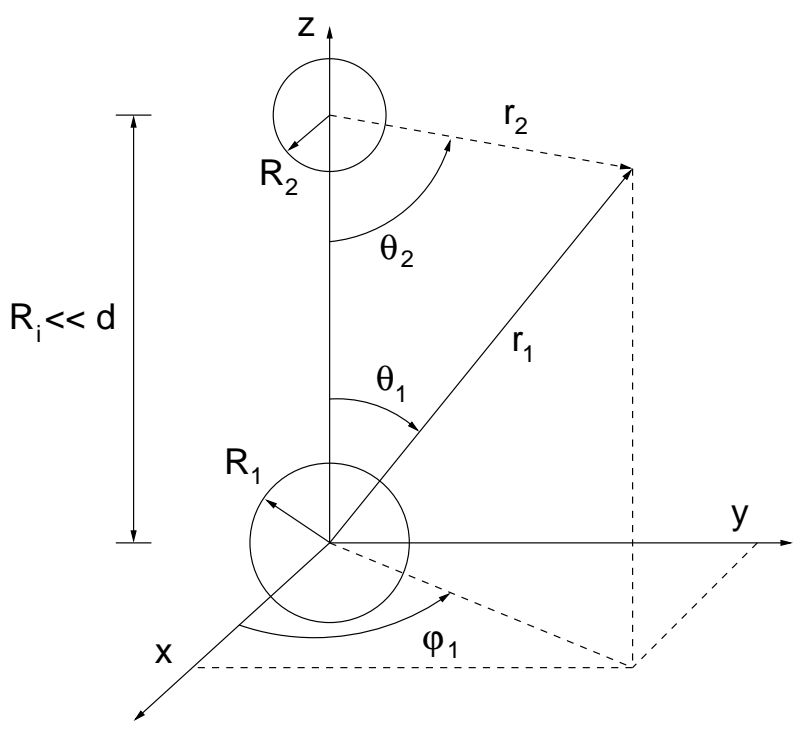

Abbildung 4.5.: Lokales, sphärisches Koordinatensystem $\left(\phi_{i}, \theta_{i}, r_{i}\right)$. O.b.d.A. werden die Blasenmittelpunkte auf die $z$-Achse gelegt. Ferner wird angenommen, daß der Abstand der Blasen $d \gg R_{i 0}$ sei.

der Dichten $\rho$ und $\rho_{g}$ der flüssigen und gasförmigen Phase gilt $\rho \gg \rho_{g}$.

Und schließslich muß betont werden, daß eine direkte Wechselwirkung der Blasen durch Kollision miteinander oder ihre Fragmentierung im Rahmen der im folgenden entwickelten Theorie nicht beschrieben werden kann.

\subsubsection{Lagrange-Formulierung}

„... berechnen wir $\mathcal{T}, \mathcal{U}$ und $\mathcal{L}$. Mehr brauchen wir von der Geometrie und Mechanik unseres Systems nicht zu wissen. Alles übrige besorgt ohne unser Zutun der Formalismus von LAGRANGE." Arnold Sommerfeld [76]

Wir betrachten ein System von $N$ masselosen, sphärischen Blasen, deren Zustand im Phasenraum bestimmt sei durch ihre Positionen $\mathbf{x}_{i}$, die Translationsgeschwindigkeiten $\mathbf{u}_{i}$, sowie die Blasenradien $R_{i}$ und die Radialgeschwindigkeiten $\dot{R}_{i}$. In der Lagrangeschen Formulierung der Bewegungsgleichungen folgen wir im Wesentlichen der Beschreibung idealer Blasenströmungen $[43,69,75,86,83]$. Es wird angenommen, daß das Geschwindigkeitsfeld $\mathbf{u}_{l}$ der Flüssigkeit wirbelfrei sei, d.h.

$$
\nabla \times \mathbf{u}_{l}=0, \quad \mathbf{x} \in \mathcal{V},
$$

wobei $\mathcal{V}$ das von der Flüssigkeit ausgefüllte Volumen bezeichnet. Das Geschwindigkeitspotential $\phi$ werde durch

$$
\mathbf{u}_{l}=\nabla \phi, \quad \mathbf{x} \in \mathcal{V},
$$

definiert. Die Kontinuitätsgleichung für ein inkompressibles Fluid führt dann zu der LaplaceGleichung

$$
\Delta \phi=0, \quad \mathbf{x} \in \mathcal{V} .
$$


Dieses elliptische Randwertproblem muß gelöst werden mit den kinematischen Randbedingungen im Unendlichen

$$
\frac{\partial \phi}{\partial n}=0, \quad \mathbf{x} \in \mathcal{S}_{\infty}
$$

und auf der Oberfläche $\mathcal{S}_{i}$ der sphärischen Blasen an den Orten $\mathbf{x}_{i}$ und mit den Radien $R_{i}$,

$$
\frac{\partial \phi}{\partial n}=\left(\mathbf{u}_{i}+\dot{R}_{i} \mathbf{n}\right) \mathbf{n}, \quad \mathbf{x} \in \mathcal{S}_{i}, \quad i=1, \ldots N,
$$

wobei $\mathbf{u}_{i}=\dot{\mathbf{x}}_{i}$ die Schwerpunktgeschwindigkeit der Blasen, $\dot{R}_{i}$ die radiale Geschwindigkeit und $\mathbf{n}$ die in das Fluid gerichtete Flächennormale bezeichnen. Es wird angenommen, daß die Blasen für alle Zeiten sphärisch sind. Da Gl. (4.28) linear in $\phi$ ist, verwenden wir als Ansatz für das Geschwindigkeitspotential

$$
\phi=-\frac{1}{4 \pi \rho} \sum_{i=1}^{N}\left(\frac{m_{i}}{r_{i}}+\frac{\mathbf{d}_{i} \mathbf{n}}{r_{i}^{2}}\right),
$$

wobei $r_{i}$ in dem lokalen, sphärischen Koordinatensystem der $i$-ten Blase gegeben ist (siehe Abb. 4.5). Mit den Randbedingungen Gln. (4.29) und (4.30) sind die Monopol- und Dipolmomente gegeben durch

$$
\begin{aligned}
m_{i}(t) & =4 \pi \rho \dot{R}_{i} R_{i}^{2}, \\
\mathbf{d}_{i}(t) & =2 \pi \rho R_{i}^{3} \mathbf{u}_{i} .
\end{aligned}
$$

Da die Blasen masselos sind, ist die kinetische Energie $\mathcal{T}$ des Systems vollständig durch die kinetische Energie

$$
\mathcal{T}=\frac{\rho}{2} \int|\nabla \phi|^{2} \mathrm{~d} \mathcal{V}
$$

des Fluids mit der Dichte $\rho$ gegeben. Durch Anwenden des Greenschen Satzes auf Gl. (4.34) und Einsetzen der Randbedingungen Gln. (4.29), (4.30) und mit dem Ansatz Gl. (4.31) folgt für die kinetische Energie schließlich

$$
\mathcal{T}=\sum_{i=1}^{N} \frac{1}{2} M_{i} \mathbf{u}_{i}^{2}+\sum_{i=1}^{N} \frac{1}{2} \mathcal{M}_{i} \dot{R}_{i}^{2}+\sum_{i=1}^{N} \sum_{i \neq j}^{N} \mathcal{U}_{i j}^{\text {eff }} .
$$

In dieser Gleichung bezeichnen

$$
\begin{aligned}
M_{i} & =\frac{2}{3} \pi \rho R_{i}^{3} \\
\mathcal{M}_{i} & =4 \pi \rho R_{i}^{3}
\end{aligned}
$$

die zusätzlichen Massen für die Translations- und Oszillationsbewegung. Die ersten beiden Summen korrespondieren zu der kinetischen Energie der Strömung durch die translatorische und oszillatorische Bewegung der Blasen. Das effektive Potential $\mathcal{U}_{i j}^{\text {eff }}=\mathcal{U}_{j i}^{\text {eff }}$ beschreibt die paarweise Wechselwirkung der Blasen und ist gegeben durch

$$
\mathcal{U}_{i j}^{e f f}=\frac{1}{4 \pi \rho}\left(\frac{m_{i} m_{j}}{\left|\mathbf{r}_{i j}\right|}+\frac{\left(m_{i} \mathbf{d}_{j}-m_{j} \mathbf{d}_{i}\right) \mathbf{r}_{i j}}{\left|\mathbf{r}_{i j}\right|^{3}}+\frac{\left(\mathbf{d}_{i} \mathbf{d}_{j}\right)\left|\mathbf{r}_{i j}\right|^{2}-3\left(\mathbf{d}_{i} \mathbf{r}_{i j}\right)\left(\mathbf{d}_{j} \mathbf{r}_{i j}\right)}{\left|\mathbf{r}_{i j}\right|^{5}}\right),
$$

wobei $\mathbf{r}_{i j}=\mathbf{x}_{i}-\mathbf{x}_{j}$ der Vektor von der Blase $j$ zu der Blase $i$ ist. Es wird angenommen, daß $\left|\mathbf{r}_{i j}\right| \gg R_{i}+R_{j}$ gilt. Die drei Terme in Gl. (4.38) entsprechen der Monopol-Monopol-, Monopol-Dipol- und Dipol-Dipol-Wechselwirkung. 
Das Gas in den Blasen wird durch ein adiabatisches Gasgesetz beschrieben. Da die Flüssigkeit inkompressibel ist, so ist die potentielle Energie des Systems allein durch die potentielle Energie $\mathcal{U}$ des Gases

$$
\mathcal{U}=4 \pi \sum_{i=1}^{N} \int_{R_{0}}^{R_{i}}\left(P\left(R_{i}^{\prime}\right)-P_{0}\right) R_{i}^{\prime 2} \mathrm{~d} R_{i}^{\prime}
$$

gegeben, wobei der Druck auf der Oberfläche der $i$-ten Blase mit dem Gleichgewichtsradius $R_{i 0}$ gegeben ist durch die Randbedingung

$$
P\left(R_{i}\right)=\left(P_{0}+\frac{2 \sigma}{R_{i 0}}\right)\left(\frac{R_{i 0}}{R_{i}}\right)^{3 \kappa}-\frac{2 \sigma}{R_{i}}, \quad i=1, \ldots, N .
$$

Hierbei bezeichnen $\kappa$ den Polytropenexponenten des Gases, $\sigma$ die Oberflächenspannung und $P_{0}$ den ambienten Druck.

Definiert man wie üblich die Lagrange-Funktion durch $\mathcal{L}=\mathcal{T}-\mathcal{U}$, so sind die Bewegungsgleichungen gegeben durch

$$
\frac{d}{d t}\left(\frac{\partial \mathcal{L}}{\partial \dot{q}_{i}^{\alpha}}\right)-\left(\frac{\partial \mathcal{L}}{\partial q_{i}^{\alpha}}\right)=F_{\alpha, i}^{\eta}+F_{\alpha, i}^{e x}+F_{\alpha, i}^{g}+F_{\alpha, i}^{b} \quad \alpha=x, y, z .
$$

Auf der linken Seite der Gl. (4.41) finden sich Kräfte, welche aus einem Potential abgeleitet werden können; für die Kräfte auf der rechten Seite ist dies nicht möglich. Zu ihnen zählen die viskose Reibung $\mathbf{F}_{i}^{\eta}$, die äußere Anregung $\mathbf{F}_{i}^{e x}$, die Auftriebskraft $\mathbf{F}_{i}^{g}$ und die primäre BjerknesKraft $\mathbf{F}_{i}^{b}$. Diese Nicht-Potential-Kräfte werden im Abschn. 4.3.3 diskutiert. Für die radialen Blasenoszillationen folgt aus Gl. (4.41) ein System modifizierter Rayleigh-Plesset-Gleichungen in der Form

$$
\begin{array}{r}
R_{i} \ddot{R}_{i}+\frac{3}{2} \dot{R}_{i}^{2}-\frac{1}{4} \mathbf{u}_{i}^{2}+\sum_{j \neq i} \frac{R_{j}\left(\ddot{R}_{j} R_{j}+2 \dot{R}_{j}^{2}\right)}{\left|\mathbf{r}_{i j}\right|} \\
+\mathcal{O}\left(\left|\mathbf{r}_{i j}\right|^{-2}\right)=\frac{1}{\rho}\left(P\left(R_{i}\right)-P_{0}\right)+\frac{F_{i}^{\eta, R}+F_{i}^{e x, R}}{4 \pi \rho R_{i}^{2}} .
\end{array}
$$

Im Grenzfall $\left|\mathbf{r}_{i j}\right| \rightarrow \infty$ entkoppelt das Gleichungssystem. Die Bewegungsgleichungen für die Translation der Blasenschwerpunkte sind entsprechend gegeben durch

$$
\dot{\mathbf{p}}_{i}-\sum_{j \neq i} \mathbf{F}_{i j}^{m, m}+\mathbf{F}_{i j}^{m, d}+\mathbf{F}_{i j}^{d, d}=\mathbf{F}_{i}^{e x, u}+\mathbf{F}_{i}^{\eta, u}+\mathbf{F}_{i}^{g}+\mathbf{F}_{i}^{b} .
$$

Für den linearen Impuls $\mathbf{p}_{i}$ gilt

$$
\mathbf{p}_{i}=2 \pi \rho\left(\frac{1}{3} R_{i}^{3} \mathbf{u}_{i}-\sum_{j \neq i} \dot{R}_{j} R_{j}^{2} R_{i}^{3} \frac{\mathbf{r}_{i j}}{\left|\mathbf{r}_{i j}\right|^{3}}+\frac{1}{2} \sum_{j \neq i} \frac{R_{i}^{3} R_{j}^{3}}{\left|\mathbf{r}_{i j}\right|^{3}}\left(\mathbf{u}_{j}-3 \frac{\mathbf{r}_{i j}\left(\mathbf{u}_{j} \mathbf{r}_{i j}\right)}{\left|\mathbf{r}_{i j}\right|^{2}}\right)\right) .
$$

Es wird an dieser Stelle ausdrücklich betont, daß der Impuls nach Gl. (4.45) der Impuls der durch die Blasen in Bewegung gesetzten Flüssigkeit ist. Da die Blasen nach Annahme masselos sind, besitzen sie auch keinen Impuls im eigentlichen Sinne. Die Gl. (4.45) beschreibt daher vielmehr den der Blase zugeordneten Impuls in gleicher Weise, wie der Blase eine zusätzliche Masse zugeordnet werden kann. Mit diesem Hinweis kann nun festgestellt werden, daß als Folge der Kopplung der Blasen, ihre Geschwindigkeiten und zugeordneten Impulse nicht proportional 
zueinander sind. Diese Eigenschaft ist wesentlich für das Verständnis der Dynamik von Blasenfeldern. Die zentrale Fragestellung ist daher die Beschreibung des Impulstransportes in diesen Systemen. Dieser Impuls wird als Kelvin-Impuls bezeichnet [7, 12]. Eine äquivalente Formulierung der Gleichung (4.45) kann mit

$$
\mathbf{p}_{i}=-\rho \int \phi \mathbf{n d} S_{i}
$$

gegeben werden.

Die Kräfte $\mathbf{F}_{i}^{m, m}, \mathbf{F}_{i}^{m, d}$ und $\mathbf{F}_{i}^{d, d}$ in Gl. (4.44) sind die aus dem effektiven Wechselwirkungspotential der kinetischen Energie folgenden Multipolwechselwirkungen. Die isotrope MonopolMonopol-Wechselwirkung ist gegeben durch

$$
\mathbf{F}_{i j}^{m, m}=\frac{m_{i} m_{j}}{4 \pi \rho} \frac{\mathbf{r}_{i j}}{\left|\mathbf{r}_{i j}\right|^{3}}
$$

und wird als sekundäre Bjerknes-Kraft bezeichnet, deren Betrag und Vorzeichen durch den Koeffizienten $m_{i} m_{j}$ bestimmt wird. Es ist üblich, den zeitlichen Mittelwert dieses Koeffizienten über eine Periode der Anregung zu betrachten. Während für lineare Blasenoszillationen eine analytische Aussage möglich ist, zeigt die numerische Simulation für hohe Anregungsamplituden die erhebliche Komplexität dieser Wechselwirkung. Die Monopol-Dipol-Wechselwirkung ist gegeben durch

$$
\mathbf{F}_{i j}^{m, d}=\frac{1}{4 \pi \rho}\left(m_{j} \frac{\mathbf{d}_{i}\left|\mathbf{r}_{i j}\right|^{2}-3 \mathbf{r}_{i j}\left(\mathbf{d}_{i} \mathbf{r}_{i j}\right)}{\left|\mathbf{r}_{i j}\right|^{5}}-m_{i} \frac{\mathbf{d}_{j}\left|\mathbf{r}_{i j}\right|^{2}-3 \mathbf{r}_{i j}\left(\mathbf{d}_{j} \mathbf{r}_{i j}\right)}{\left|\mathbf{r}_{i j}\right|^{5}}\right) .
$$

Und schließlich ist die Wechselwirkung zweier sich in dem Fluid bewegender Blasen durch die Dipol-Dipol-Kraft

$$
\mathbf{F}_{i j}^{d, d}=\frac{3}{4 \pi \rho}\left(\frac{\mathbf{r}_{i j}\left(\mathbf{d}_{i} \mathbf{d}_{j}\right)+\mathbf{d}_{i}\left(\mathbf{d}_{j} \mathbf{r}_{i j}\right)+\mathbf{d}_{j}\left(\mathbf{d}_{i} \mathbf{r}_{i j}\right)}{\left|\mathbf{r}_{i j}\right|^{5}}-\frac{5 \mathbf{r}_{i j}\left(\mathbf{d}_{i} \mathbf{r}_{i j}\right)\left(\mathbf{d}_{j} \mathbf{r}_{i j}\right)}{\left|\mathbf{r}_{i j}\right|^{7}}\right)
$$

gegeben. Die Eigenschaften der Kräfte (4.47), (4.48) und (4.49) werden in dem Abschn. 4.3.5 diskutiert. Welche dieser Wechselwirkungen für die Bewegung einen wesentlichen Beitrag liefert, ist dadurch bestimmt, wie sie zu dem effektiven Wechselwirkungspotential beitragen. Dies kann für einzelne Bewegungsformen sehr unterschiedlich sein und muß daher im Einzelfall geprüft werden.

\subsubsection{Dissipation}

Die Natur der Dissipationsprozesse, die zu einer Dämpfung der radialen Schwingung und der linearen Translationsbewegung führen, ist überaus komplex [16, 20, 66, 19]. Die Visosität, die Kompressibilität und die thermische Leitfähigkeit der Flüssigkeit dämpfen die Bewegung der Blasen. Die relative Bedeutung dieser Mechanismen ist abhängig von dem Ruheradius der Blasen und/oder der Frequenz der Anregung. So ist die viskose Dämpfung unabhängig von der Frequenz, wächst aber stark an für kleine Blasen. Die akustische Dämpfung ist dominierend für große Blasen und hohe Frequenzen. Die thermische Dämpfung hingegen ist für niedrige Frequenzen der wesentliche Beitrag der Dämpfung (vgl. Abb. 4.6). Im Rahmen einer linearen Theorie werden die Beträge dieser Dissipationsmechanismen zu einer effektive Viskosität zusammengefaßt. 


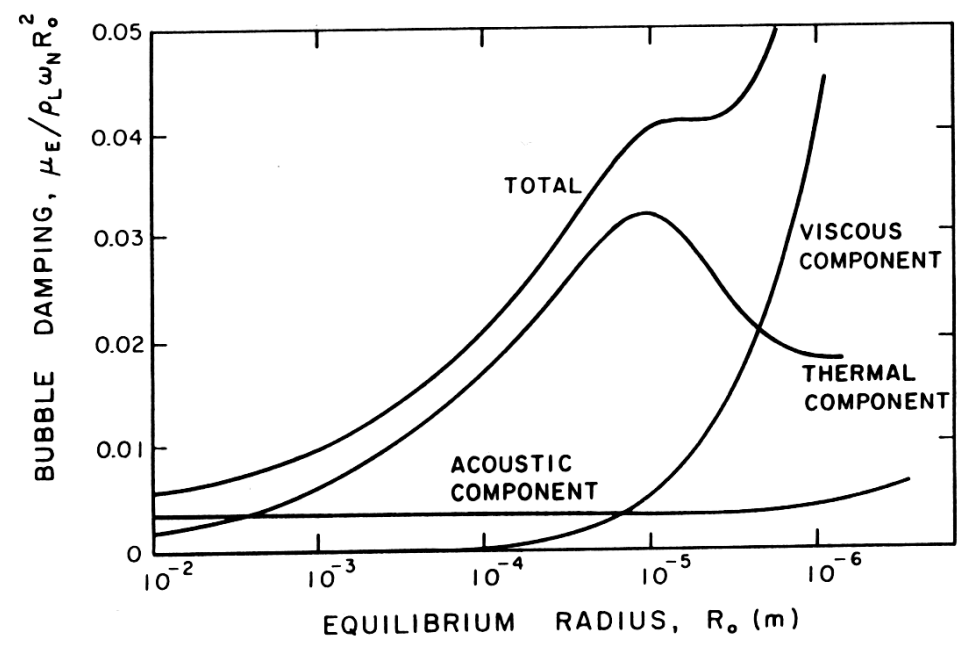

Abbildung 4.6.: Viskose, thermische und akustische Dämpfung der Blasenoszillation als Funktion des Ruheradius $R_{0}$. Die Komponenten können zu einer effektiven Viskosität zusammengefaßt werden. Für Blasen mit Ruheradien von 1-10 $\mu \mathrm{m}$ ist die viskose und thermische Dämpfung dominierend [16]. Die effektive Viskosität $\mu_{e}$ ist entdimensionalisiert aufgetragen.

Im Rahmen des hier entwickelten Modells wird vereinfachend angenommen, daß die Dissipation hinreichend durch die Berücksichtigung der Viskosität beschrieben ist. Die viskosen Reibungskräfte $\mathbf{F}_{i}^{\eta}$ können mit Hilfe der Rayleighschen Dissipationsfunktion $\mathcal{F}$ bestimmt werden [44, 24]:

$$
F_{\alpha, i}^{\eta}=-\frac{\partial \mathcal{F}}{\partial \dot{q}_{i}^{\alpha}} \quad \text { mit } \quad \alpha=x, y, z .
$$

Die Dissipationsfunktion beschreibt die Intensität der Energiedissipation des mechanischen Systems in der Form

$$
\frac{d E}{d t}=-2 \mathcal{F} .
$$

Die Energiedissipation in einer inkompressiblen, viskosen Potentialströmung mit der Zähigkeit $\eta$ hat die besonders einfache Form [45]

$$
\frac{d E}{d t}=-\eta \int \nabla \mathbf{u}_{l}^{2} \mathrm{~d} \mathcal{S} .
$$

Mit Gl. 4.51 folgt daher für die Dissipationsfunktion

$$
\mathcal{F}=\sum_{i}\left(8 \pi \eta R_{i} \dot{R}_{i}^{2}+6 \pi \eta R_{i} \mathbf{u}_{i}^{2}\right) .
$$

Der erste Term der Summe korrespondiert zu der Dämpfung der radialen Oszillation, während der zweite Term der Dämpfung der Translationsbewegung entspricht. In Gl. (4.53) sind Terme, die durch die Kopplung hervorgerufen sind, vernachlässigt worden. Mit Gl. (4.50) ergibt sich damit zunächst für die Reibungskraft der Translationsbewegung

$$
\mathbf{F}_{i}^{\nu, u}=-12 \pi \eta R_{i} \mathbf{u}_{i}
$$

in Übereinstimmung mit $[49,54]$. Die Reibungskraft für die radiale Oszillation ist entsprechend gegeben durch

$$
\mathbf{F}_{i}^{\nu, r}=-16 \pi \eta R_{i} \dot{R}_{i} .
$$




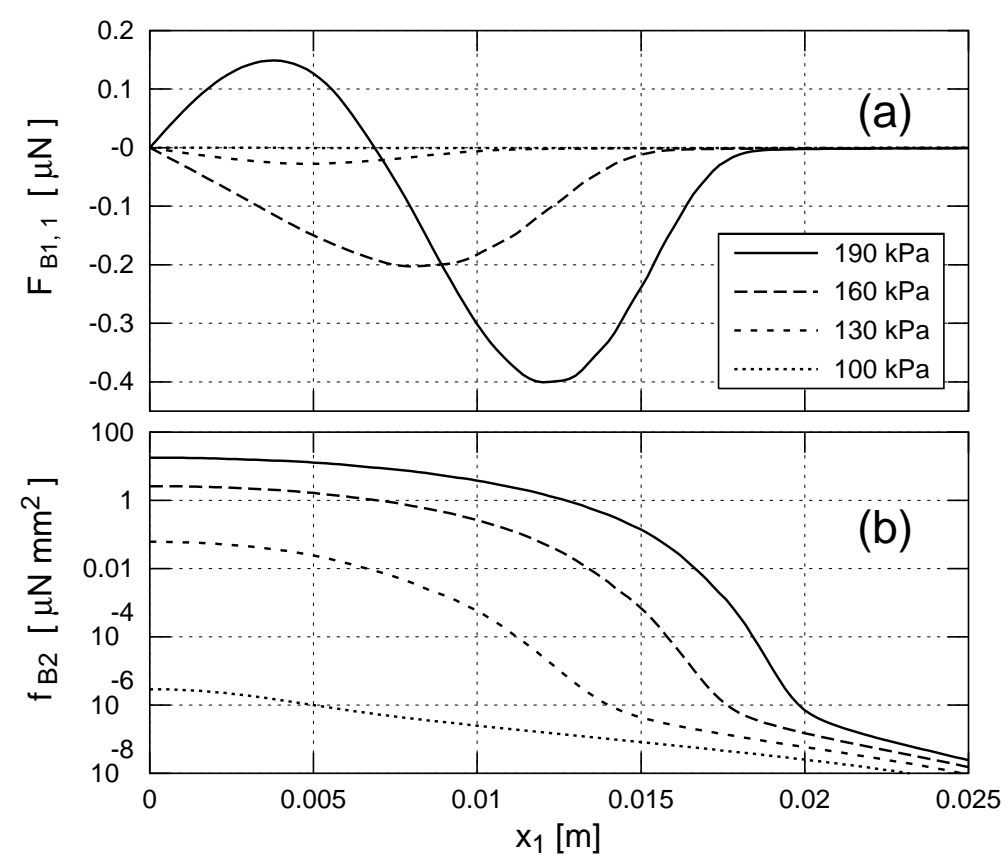

Abbildung 4.7.: Koeffizienten der primären und sekundären Bjerknes-Kraft.

\subsection{3. Äußere Kräfte}

Neben den in Abschn. 4.3.2 beschriebenen dissipativen Kräften wirken auf die Blasen auch weitere externe Kräfte. Hierzu zählen die Auftriebskraft, die primäre Bjerknes-Kraft und die externe Anregung. Die Auftriebskraft ist gegeben durch

$$
\mathbf{F}_{i}^{g}=\frac{4 \pi}{3} \rho R_{i}^{3} \mathbf{g} \quad \text { mit } \quad \mathbf{g}=g \hat{e}_{z} .
$$

Auf die Blase wirkt weiterhin die primäre Bjerknes-Kraft [11]

$$
\mathbf{F}_{i}^{b, u}=-\frac{4 \pi}{3} \nabla p_{a} R_{i}^{3}
$$

Ein akustisches Stehwellenfeld $P_{a}\left(\mathbf{x}_{i}, t\right)$ mit einer Wellenlänge, die groß im Vergleich zu dem Blasenradius sei, führt zu einer externen Anregung der Blasen und einer instantanen primären Bjerknes-Kraft $[64,55]$

$$
\mathbf{F}_{i}^{e x, R}=-4 \pi R_{i}^{2} p_{a}\left(\mathbf{x}_{i}\right) \sin (\omega t) \mathbf{n} \quad \text { und } \quad \mathbf{F}_{i}^{b, u}=-\frac{4 \pi}{3} \nabla p_{a}\left(\mathbf{x}_{i}\right) R_{i}^{3} .
$$

\subsubsection{Translation}

In diesem Abschnitt werden Lösungen der Gln. (4.42), (4.44) und (4.45) für die eindimensionale Translationsbewegung einer einzelnen Blase diskutiert. Von besonderem Interesse ist die Kopplung von radialer Oszillation und Translation.

Zunächst sei jedoch angenommen, daß der Radius konstant sei $\left(R=R_{0}\right)$. Wirken auf die Blase nur die Auftriebskraft und die viskose Reibung, so folgt aus den Gln. (4.44) und (4.45) zunächst

$$
\dot{u}=-\frac{18 \nu}{R_{0}^{2}} u+2 g
$$




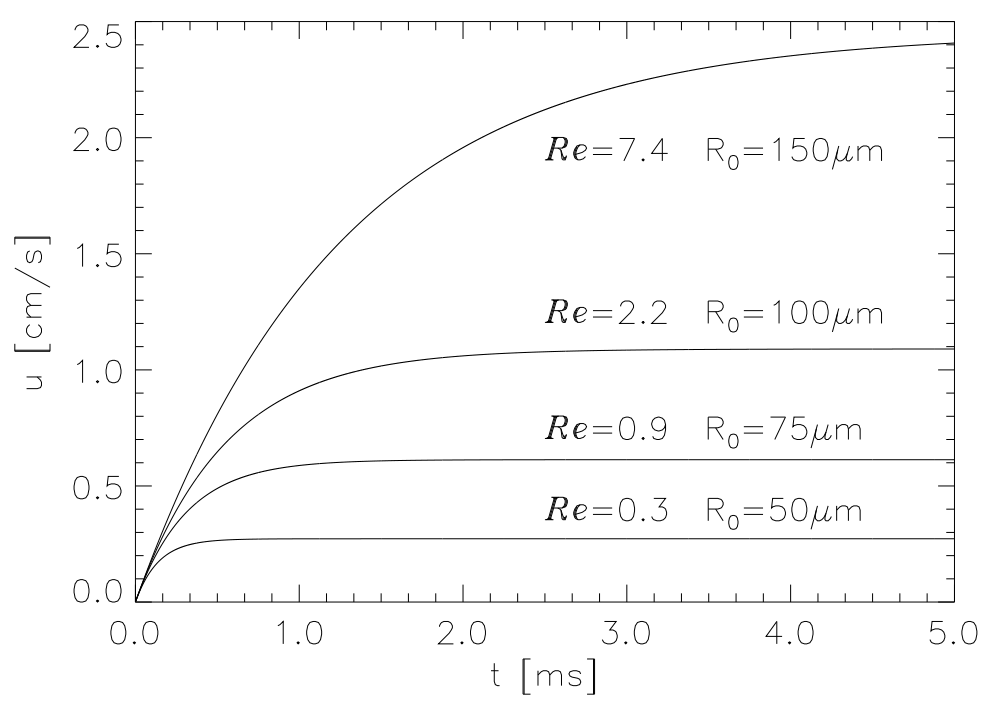

Abbildung 4.8.: Geschwindigkeit $u(t)$ für verschiedene Radien $R_{0}$ entsprechend Gl. (4.60). Es sind jeweils die asymptotischen Reynoldszahlen $\mathcal{R} e^{\infty}$ nach Gl. (4.63) angegeben.

und nach Integration unmittelbar

$$
u(t)=u^{\infty}\left(1-e^{-t / t_{\tau}}\right) \quad \text { mit } \quad u(0)=0 .
$$

Ist die Blase zum Zeitpunkt $t=0$ in Ruhe, so erreicht sie für $t \rightarrow \infty$ die asymptotische Aufstiegsgeschwindigkeit

$$
u^{\infty}=\lim _{t \rightarrow \infty} u(t)=\frac{R_{0}^{2} g}{9 \nu} .
$$

Die Blasenbewegung ist dabei charakterisiert durch eine typische Relaxationszeit

$$
t_{\tau}=\frac{R_{0}^{2}}{18 \nu}
$$

Bemerkenswert ist, daß die $t_{\tau}$ nur von der kinematischen Viskosität und von dem Blasenradius abhängt. Für Blasen in Wasser bei $20^{\circ} \mathrm{C}$ ergeben sich für verschiedene Radien $R_{0}$ die folgenden Zahlenwerte für die Relaxationszeit $t_{\tau}$ :

$$
\begin{array}{c|ccccc}
R_{0} & 1 \mu \mathrm{m} & 10 \mu \mathrm{m} & 100 \mu \mathrm{m} & 1 \mathrm{~mm} & 1 \mathrm{~cm} \\
\hline t_{\tau} & 5.5 \cdot 10^{-8} \mathrm{~s} & 5.5 \cdot 10^{-6} \mathrm{~s} & 5.5 \cdot 10^{-4} \mathrm{~s} & 5.5 \cdot 10^{-2} \mathrm{~s} & 5.5 \mathrm{~s}
\end{array}
$$

Im asymptotischen Grenzfall erreichen die Blasen dann die Reynoldszahl

$$
\mathcal{R} e^{\infty}=\frac{2 g R_{0}^{3}}{9 \nu^{2}} \quad \text { mit } \quad \nu=\frac{\eta}{\rho} .
$$

Beispiele einer solchen eindimensionalen Aufstiegsbewegung sind für verschiedene Reynoldszahlen in Abb. 4.8 gezeigt. 

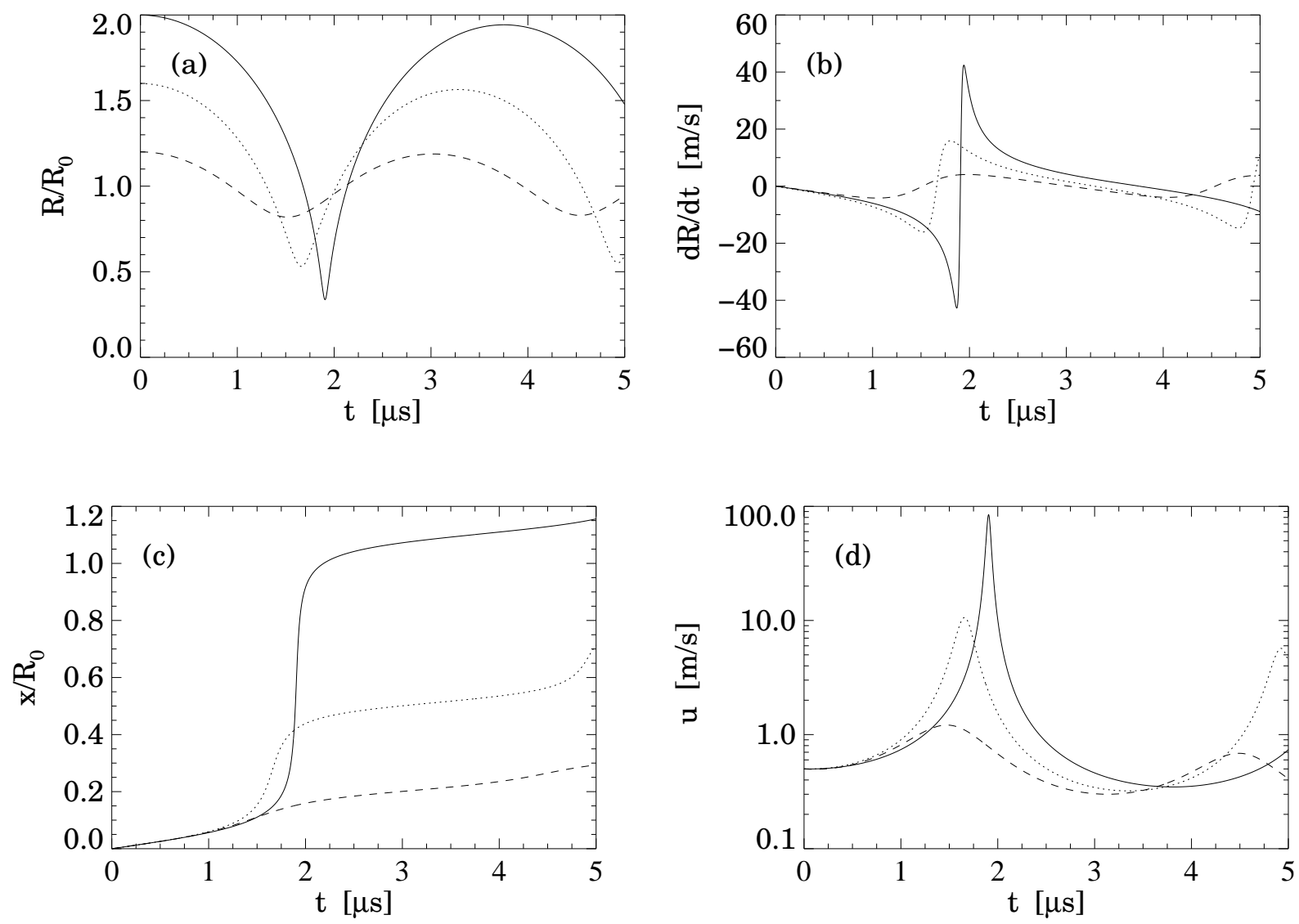

Abbildung 4.9.: Kopplung von Translation und Oszillation einer Einzelblase. Numerische Lösung der Gln. (4.64) und (4.65) mit den Anfangsbedingungen $R(0)=\alpha R_{0}$ mit $R_{0}=10 \mu \mathrm{m}$ und $\alpha=1.2,1.6$ und 2.0 sowie jeweils $u(0)=0.5 \mathrm{~m} / \mathrm{s}$. Die Radien in (a) und die Ortskoordinate in (c) sind mit dem Ruheradius $R_{0}$ entdimensionalisiert.

Ein weiterer einfacher, jedoch aufschlußreicher Grenzfall der Bewegungsgleichungen ist die Kopplung von freier, radialer Oszillation und linearer Translation. Die Gleichungen für den Radius $R(t)$ und den Blasenschwerpunkt $x(t)$ sind dann gegeben durch

$$
\begin{aligned}
R \ddot{R}+\frac{3}{2} \dot{R}^{2} & =\frac{1}{\rho}\left(P(R)-P_{0}\right)-4 \mu \frac{\dot{R}}{R}, \\
\ddot{x} & =-3 \frac{\dot{R}}{R}-18 \mu \frac{\dot{x}}{R^{2}},
\end{aligned}
$$

wobei für den Druck $P(R)$ die Gl. (4.40) gelte. In Abb. 4.9 sind drei Lösungen der Gl. (4.64) und (4.65) dargestellt. Die Blasen werden bei $t=0$ auf den 1.2-, 1.6- bzw. 2.0-fachen Ruheradius $R_{0}=10 \mu \mathrm{m}$ aufgezogen und impulsiv mit $\dot{x}(0)=0.5 \mathrm{~m} / \mathrm{s}$ in Bewegung gesetzt. Abb. 4.9 (a) zeigt die freien radialen Oszillationen, die durch viskose Reibung schwach gedämpft werden. Abb. 4.9 (b) zeigt die entsprechende Translationsbewegung.

In der Kollapsphase der radialen Oszillation nimmt die Translationgeschwindigkeit deutlich zu: Je stärker der Kollaps, desto drastischer ist die lineare Beschleunigung. Diese Dynamik ist eine 

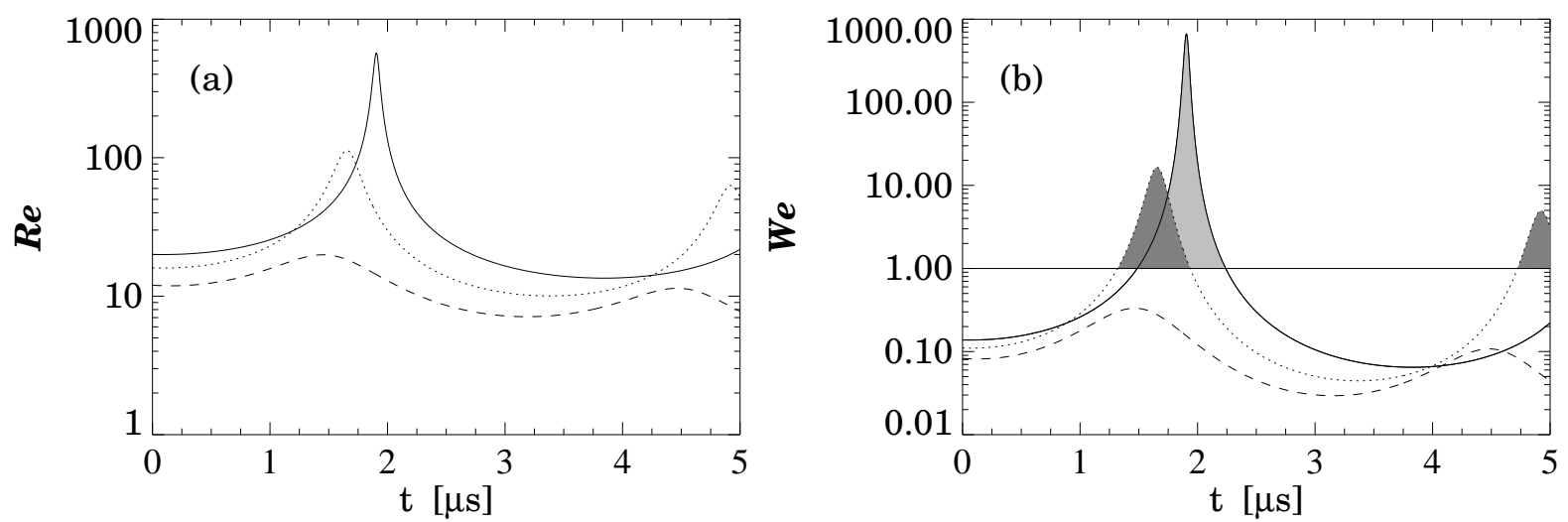

Abbildung 4.10.: Die Reynoldszahl $\mathcal{R} e$ (Gl. (4.2)) und die Weberzahl $\mathcal{W} e$ (Gl. (4.4)) für die in Abb. 4.9 dargestellten Trajektorien. Für $\mathcal{W} e \gg 1$ wird der Blasenkollaps asphärisch; diese Bereiche sind schattiert dargestellt.

einfache Konsequenz aus der Impulserhaltung der Translationsbewegung. Zum Zeitpunkt $t=0$ hat die Blase den linearen Impuls

$$
p(0)=m(0) u(0)
$$

mit der zusätzlichen und insbesondere zeitabhängigen Masse $m(t)=2 / 3 \pi \rho R(t)^{3}$. Eine kollabierende Blase reduziert daher ihre translatorische Masse in der dritten Potenz des Radius': Ändert sich der Radius auf 1/10 des Maximalradius, so reduziert sich die Masse entsprechend um den Faktor 1/1000. Vernachlässigen wir zunächst den Einfluß viskoser Reibung, so folgt aus $\dot{p}(t)=0$, daß eine Blase in der Kollapsphase eine erhebliche Beschleunigung erfahren muß. Für die Modellierung ist nun von entscheidender Bedeutung, in welchem hydrodynamischen Regime sich diese Bewegung abspielt und wie eine Aussage über die Stabilität der Blase gewonnen werden kann. Hierzu werden die in den Gln. (4.2) und (4.4) definierten Reynolds- und Weberzahlen für die Beispiele aus Abb. 4.9 in Abb. 4.10 dargestellt. Die Weberzahl, die das Verhältnis von Trägheitskräften zu Kräften der Oberflächenspannung beschreibt, kann als Kriterium für Sphärizität verwendet werden. Für $\mathcal{W} e \ll 1$ dominieren die Kräfte der Oberflächenspannung, die bestrebt sind, die Blase in sphärischer Form zu halten. Für $\mathcal{W} e \gg 1$ überwiegen hingegen die Trägheitskräfte, was mit dem Verlust der Sphärizität einhergeht. Bei der Diskussion der Kennzahlen muß beachtet werden, daß sie als Funktionen des Radius und der Translationsgeschwindigkeit nun selbst Funktionen der Zeit sind. Abb. 4.10 (b) zeigt den Zeitverlauf der Weberzahl. Nur für eines der drei dargestellten Beispiele ist die Weberzahl immer deutlich kleiner eins. In den beiden anderen Fällen, in denen die Blase erheblich stärker kollabiert, steigt die Weberzahl zeitweise um Größenordnungen über den kritischen Wert eins an. Daher ist in diesen Fällen von einem Verlust der Sphärizität auszugehen. Die Reynoldszahl beschreibt das Verhältnis von konvektivem zu diffusivem Impulstransport. Auch hier ist der Zeitverlauf von charakteristischen Schwankungen begleitet. Es muß daher für die jeweiligen Simulationsparameter überprüft werden, ob die Modellannahmen im Verlauf der Simulation erfüllt bleiben. Die hier diskutierte Lösung der Modellgleichungen ist in qualitativer Übereinstimmung mit den experimentellen Befunden in $[17,47]$. 

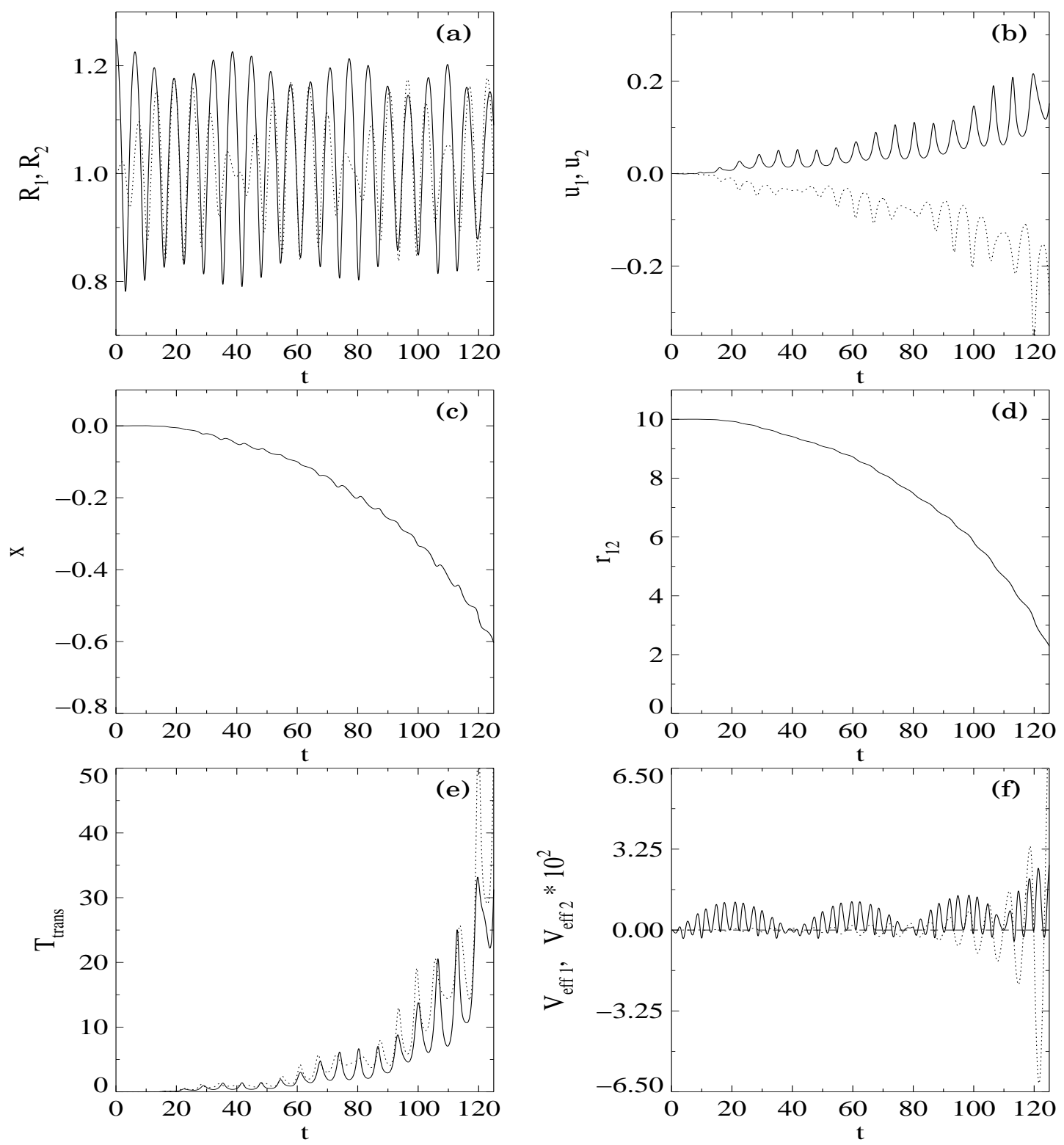

Abbildung 4.11.: (a) Dimensionslose Radien $R_{1} / R_{01}$ (durchgezogene Kurve) und $R_{2} / R_{02}$ (gestrichelte Kurve) mit den Gleichgewichtsradien $R_{01}=0.1 \mathrm{~mm}$ und $R_{02}=0.09 \mathrm{~mm}$ und den Anfangsbedingungen $R_{1}(0)=1.5 \cdot R_{01}$ und $R_{2}(0)=R_{02}$. (b) Geschwindigkeiten $u_{1}$ und $u_{2}$ der Blasenschwerpunkte. (c) Dimensionsloser Mittelpunktsabstand $x$ der Blasen. (d) Dimensionsloser Abstand $R_{12}$ der Blasen. (e) Kinetische Energie der Translationsbewegung. (f) Effektives Potential der Monopol-Monopol-, Monopol-Dipol und DipolDipol-Wechselwirkung. Die Zeit ist in Einheiten von $\omega_{01} t$ aufgetragen. 

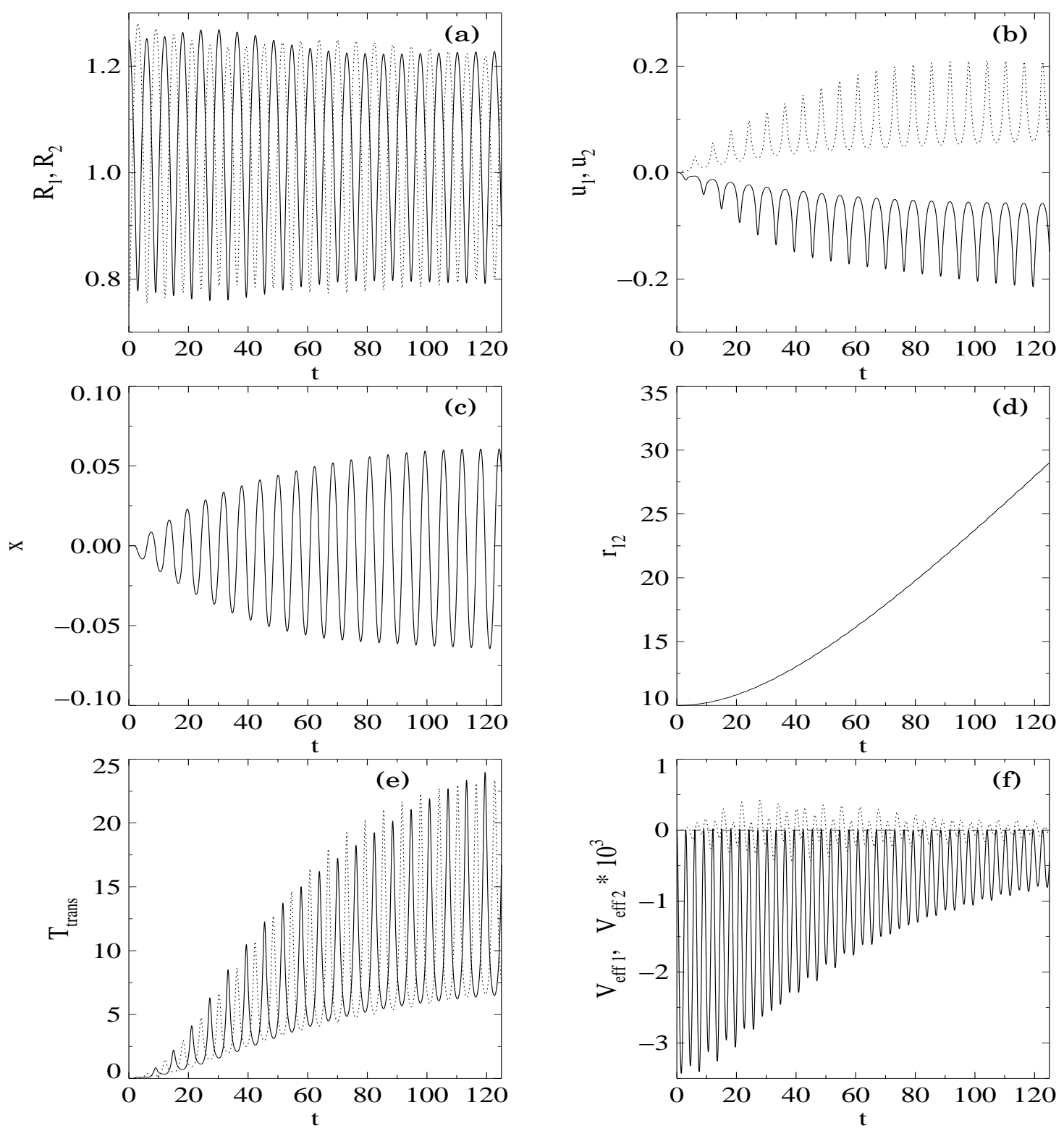

Abbildung 4.12.: Darstellung wie Abb. 4.11, jedoch für Blasen mit Ruheradien $R_{01}=R_{02}=0.1$ $\mathrm{mm}$ und mit den Anfangsbedingungen $R_{1}(0)=1.25 \cdot R_{01}$ und $R_{2}(0)=$ $0.75 \cdot R_{02}$. 


\subsubsection{Wechselwirkung}

Die Multipolwechselwirkung niedrigster Ordnung ist die isotrope Monopol-MonopolWechselwirkung. Die ihr entsprechende Kraft wird in der Literatur als sekundäre Bjerknes-Kraft bezeichnet [11]. In [87] wird der Lagrange-Formalismus für die sphärischen Ozillationen eines Blasenpaares in einer inkompressiblen Potentialströmung angewendet und ein System gekoppelter, nichtlinearer gewöhnlicher Differentialgleichungen bestimmt. Die sekundäre Bjerknes-Kraft wird aus der Wechselwirkungsenergie bestimmt. Das Ergebnis dieser Arbeit ist die Identifikation von drei Typen der Wechselwirkung auf der Grundlage der linearisierten Bewegungsgleichungen. Neben der für alle Abstände $\left|\mathbf{r}_{i j}\right|$ entweder ausschließlich repulsiven oder ausschließlich attraktiven Kraft werden Fixpunkte gefunden, für die das Vorzeichen der Kraft wechselt. Es wird ein stabiler Fixpunkt $\left|\mathbf{r}_{0}\right|$ identifiziert, für den die Kraft für $\left|\mathbf{r}_{i j}\right|<\left|\mathbf{r}_{0}\right|$ repulsiv und für große Abstände $\left|\mathbf{r}_{i j}\right|>\left|\mathbf{r}_{0}\right|$ attraktiv ist. Für den zweiten Fixpunkt ist die Kraft für $\left|\mathbf{r}_{i j}\right|<\left|\mathbf{r}_{0}\right|$ attraktiv und für $\left|\mathbf{r}_{i j}\right|>\left|\mathbf{r}_{0}\right|$ repulsiv und der Fixpunkt daher instabil. Da die Theorie nur lineare Oszillationen betrachtet, ist das Vorzeichen der Wechselwirkung nur abhängig von dem Verhältnis der anregenden Frequenz $\omega$ zu den linearen Resonanzfrequenzen $\omega_{0 i}$ bzw. den Normalmoden des gekoppelten Systems und nicht von dem Anregungsdruck $p_{a}$. In [62] wird ein Modell der Blasenwechselwirkung auf der Basis eines allgemeinen Virialtheorems gegeben, das abgeleitet wird vom Bernoulli-Integral für eine nicht-viskose, unstetige Potentialströmung. Das System nichtlinearer, gewöhnlicher Differentialgleichungen für die radialen Blasenoszillationen und die Translationsbewegung wird numerisch gelöst. Als Erweiterung der linearen Theorie wird in diesem Modell bei einer Erhöhung der Anregungsamplitude ein Vorzeichenwechsel der Wechselwirkung beobachtet.

$$
\mathbf{F}_{i j}^{m, m}=\frac{m_{i} m_{j}}{4 \pi \rho} \frac{\mathbf{r}_{i j}}{\left|\mathbf{r}_{i j}\right|^{3}} .
$$

Der Betrag und das Vorzeichen dieser Kraft sind Funktionen der Monopol-Momente $m_{i} m_{j}$ (siehe Gl. (4.32)). Für lineare Blasenschwingungen ist die sekundäre Bjerknes-Kraft gegeben durch

$$
\mathbf{F}_{12}^{m, m}=-\frac{\rho \omega^{2} R_{01}^{3} R_{01}^{3}}{\left|\mathbf{r}_{i j}\right|^{3}} r_{1} r_{2} \cos \left(\psi_{1}-\psi_{2}\right) .
$$

Im linearen Regime folgt aus Gl. (4.68), daß Blasen, deren Ruheradien beide größer oder beide kleiner als der lineare Resonanzradius sind, eine attraktive Wechselwirkung erfahren. Ist hingegen ein Gleichgewichtsradius kleiner und der zweite größer als der Resonanzradius, so ist die Wechselwirkung repulsiv. Die lineare Resonanzfrequenz der Blase mit Ruheradius $R_{0 i}$ wird als Minnaert-Frequenz bezeichnet und ist gegeben durch

$$
\omega_{0 i}^{2}=\frac{1}{\rho R_{0 i}^{2}}\left(3 \kappa p_{0}-\frac{2 \sigma}{R_{0}}\right) .
$$

Wir betrachten den Fall freier Oszillationen. Zur Validierung der Numerik und zum Vergleich mit Literaturergebnissen wird das Modellgleichungssystem mit den Anfangsbedinungen nach [62] (Fig. 5 und Fig. 7) gelöst. Die numerischen Lösungen aus [62] ergeben sich als eindimensionaler Grenzfall des hier vorgestellten, dreidimensionalen Modells. Die Ergebnisse zeigen sowohl eine sehr gute qualitative als auch quantitative Übereinstimmung. In Abb. 4.11 werden zwei Blasen mit den Ruheradien $R_{01}=0.1 \mathrm{~mm}$ und $R_{02}=0.09 \mathrm{~mm}$ betrachtet, die sich anfänglich im Abstand $\left|r_{12}\right|=10 R_{01}=1 \mathrm{~mm}$ befinden. Die Anfangsbedingung für Blase 1 ist $R_{1}(0) / R_{01}=1.25$, während Blase 2 sich im Gleichgewicht befindet $\left(R_{2}(0)=R_{02}\right)$. Beide Blasen befinden sich zunächst in Ruhe. In Abb. 4.11 sind die Radien $R_{i}$ mit dem Ruheradius normiert und die Zeit in Einheiten von $\omega_{01} t$ aufgetragen. Abb. 4.11 (a) zeigt den typischen Verlauf eines gekoppelten Oszillatorsystems, bei dem Energie von Blase 1 auf Blase 2 übertragen wird. Die anfänglich in Ruhe befindlichen Blasen werden durch ihre Wechselwirkung in Bewegung 
gesetzt (Abb. 4.11 (b)). Dabei oszilliert die Geschwindigkeit in der in Abs. 4.3 .4 charakterisierten Weise. In Abb. 4.11 (c,d) ist der Blasenabstand bzw. der Mittelpunkt der Ortskoordinaten aufgetragen. Die Wechselwirkung ist deutlich attraktiv und der Abstand verringert sich rasch von 10 auf 2 Ruheradien. Die kinetische Energie der Translationsbewegung ist in Abb. 4.11 (e) als Funktion der Zeit dargestellt. Sie ist anfangs gleich Null und wächst im Mittel an. Und schließlich sind die Beträge des effektiven Potentials in Abb. 4.11 (f) dargestellt. Die einzelnen Terme unterscheiden sich hier um jeweils 2 bis 3 Größenordnungen. Die Monopol-MonopolWechselwirkung ist hierbei der klar dominierende Term. Er zeigt die auch in den Radius-ZeitKurven erkennbare Schwebung. Die Monopol-Dipol-Wechselwirkung ist in Abb. 4.11 (f) reskaliert. Das sowohl Monopol-Dipol- als auch Dipol-Dipol-Wechselwirkung in diesem Beispiel keine Rolle spielen, beruht auf der Tatsache, daß die Blasen nur eine geringe Schwerpunktsgeschwindigkeit besitzen und daher die Dipolmonente klein sind.

Abb. 4.12 zeigt ein weiteres Beispiel freier Blasenoszillation. Beide Blasen haben hier die gleichen Ruheradien $R_{01}=R_{02}=0.1 \mathrm{~mm}$, aber die Oszillationen beginnen mit entgegengesetzten Phasenlagen durch die Wahl $R_{1}(0) / R_{01}=1.25$ und $R_{2}(0) / R_{02}=0.75$. Wie aus der linearen Theorie erwartet, ist die Wechselwirkung in diesem Fall repulsiv.

Die Dipol-Dipol-Wechselwirkung ist die in idealen Blasenströmungen vorherrschende Wechselwirkung. Die Wechselwirkung zweier Dipole in einer idealen Potentialströmung wird in einer Vielzahl von Arbeiten betrachtet [4, 25, 26, 27, 38, 40, 41, 42, 72, 77, 81, 85, 6, 30, 58, 78, 75]. Für die Kraft zwischen den Dipolen mit den Dipolmomenten $\mathbf{d}_{i}$ und $\mathbf{d}_{j}$ gilt

$$
\mathbf{F}_{i j}^{d, d}=\frac{3}{4 \pi \rho}\left(\frac{\mathbf{r}_{i j}\left(\mathbf{d}_{i} \mathbf{d}_{j}\right)+\mathbf{d}_{i}\left(\mathbf{d}_{j} \mathbf{r}_{i j}\right)+\mathbf{d}_{j}\left(\mathbf{d}_{i} \mathbf{r}_{i j}\right)}{\left|\mathbf{r}_{i j}\right|^{5}}-\frac{5 \mathbf{r}_{i j}\left(\mathbf{d}_{i} \mathbf{r}_{i j}\right)\left(\mathbf{d}_{j} \mathbf{r}_{i j}\right)}{\left|\mathbf{r}_{i j}\right|^{7}}\right) .
$$

Um die Dipol-Dipol-Wechselwirkung an einem einfachen Beispiel zu diskutieren, wird die zweidimensionale Bewegung eines Blasenpaares unter Einfluß des Auftriebs betrachtet. O.b.d.A. wird angenommen, daß die Bewegung auf die x-z-Ebene beschränkt sei. Der Schwerpunkt der ersten Blase wird als Ursprung des in Abb. 4.13 dargestellten mitbewegten Polarkoordinatensystems gewählt, in dem mit $\Theta$ der Winkel mit der z-Achse und mit $d$ der Abstand definiert sind. In Abb. 4.14 sind Beispiele der Trajektorien dargestellt. Um die Ergebnisse unseres Modells mit den Ergebnissen der Literatur zu vergleichen, wurden die Anfangsbedingungen aus [40] gewählt. Abhängig von den gewählten Anfangsbedingungen ist die Dipol-Dipol-Wechselwirkung zunächst attraktiv oder repulsiv. Es wirkt jedoch auf das Blasenpaar ein Drehmoment, das sie in eine relative Position quer zur Strömung bewegt. Für $\Theta=0^{\circ}$ und $\Theta=180^{\circ}$ finden wir einen instabilen Fixpunkt. Jede kleine Strörung bewegt die Blase aus diesem Gleichgewicht. Daher sind rein axialsymmetrische Betrachtungen der Dipol-Dipol-Wechselwirkung von geringem praktischem Nutzen [40].

Für den einfachen Fall der Dipol-Dipol-Wechselwirkung kann gleichfalls das Konzept der zusätzlichen Masse an diesem konkreten Beispiel betrachtet werden. Die Matrix der zusätzlichen Masse hat nun die einfache Form [69]:

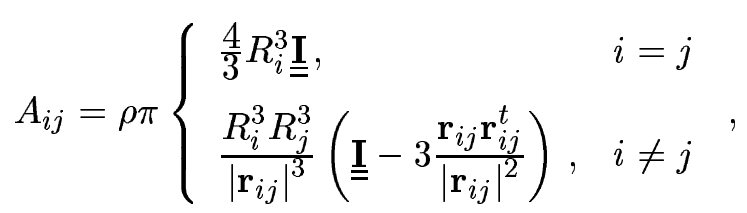

wobei $\underline{I}$ die $3 \times 3$-Einheitsmatrix bezeichnet. Die Diagonalelemente der Matrix entsprechen den zusätzlichen Massen einer einzelnen Blase, wenn sie sich in einem unendlich ausgedehnten Fluid bewegen. Die Submatrizen für $i \neq j$ beruhen auf der Kopplung der Blasen, die einen effektiven Beitrag zu der zusätzlichen Masse des Gesamtsystems liefert. Mit Hilfe der Matrix Gl. (4.71) 


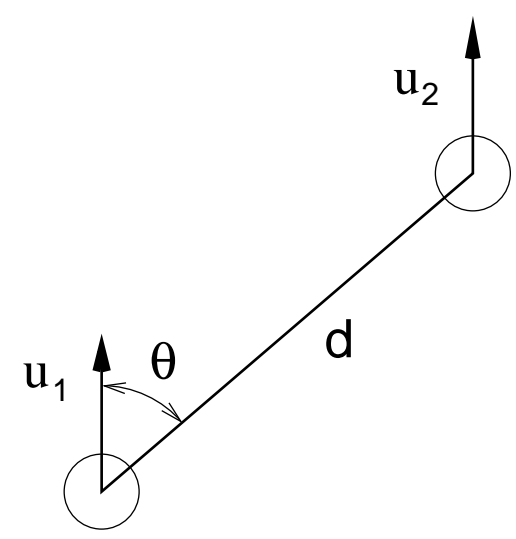

Abbildung 4.13.: Relativkoordinaten $\Theta$ und $d$ für die zweidimensionale Bewegung eines Blasenpaares mit den Geschwindigkeiten $u_{1}$ und $u_{2}$.

kann nun die Bewegung der Blasen charakterisiert werden. Hierzu wird der Koeffizient

$$
c_{m}=\frac{\mathbf{u} \cdot \underline{\underline{\mathbf{A}} \cdot \mathbf{u}}}{\mathbf{u} \cdot \mathbf{u}}
$$

eingeführt [86]. Für das hier gezeigte Beispiel der Dipol-Dipol-Wechselwirkung zeigt die numerische Simulation des Koeffizienten Gl. (4.72), daß die Bewegung der Blasen die zusätzliche Massen des Blasensystems maximiert.

\subsection{Vergleich der Modelle}

In diesem Abschnitt werden das in Abschn. 4.2 vorgestellte Kontinuumsmodell und das im Rahmen dieser Arbeit entwickelte Partikelmodell aus Abschn. 4.3 kritisch miteinander verglichen.

Grundlage der Beschreibung der makroskopischen Eigenschaften akustischer Kavitationsblasenfelder im Rahmen des Kontinuumsmodells ist die Wellengleichung (4.8). In dieser Gleichung werden lineare Blasenoszillationen vorausgesetzt. Damit ist der Anwendungsbereich der auf ihr beruhenden Theorie begrenzt auf kleine Schalldrücke. Die Nichtlinearität des Kontinuumsmodells beruht auf der Kopplung von Blasendichte und Schallfeld. Das Kontinuumsmodell beschreibt die Selbstfokussierung der ebenen Schallwelle und die Selbstkonzentration der Blasen. Die langsam veränderliche Schallfeldamplitude wird durch eine Amplitudengleichung vom Typ der Nichtlinearen Schrödinger-Gleichung beschrieben. Es ist damit gelungen, die Ausbreitung solitärer Wellen in Blasen-Flüssigkeits-Gemischen zu beschreiben und damit die Zuordnung dieses Systems zur Modellklasse strukturbildender, räumlich ausgedehnter Systeme zu leisten [51]. Die primäre Instabilität, die zur Ausbildung der solitären Strukturen führt, kann im Rahmen einer linearen Stabilitätsanalyse charakterisiert werden.

Das Kontinuumsmodell beschreibt die Ausbreitung ebener Wellen. Eine Verallgeimenerung dieses Ansatzes auf die in den experimentellen Aufbauten üblichen stehenden Wellen wirft konzeptionelle Schwierigkeiten im Rahmen der verwendeten Mehrskalenanalyse auf. Für eine detaillierte Diskussion diese Sachverhaltes siehe [79]. Damit wird die experimentelle Anwendbarkeit und damit die Möglichkeit einer experimentellen Verifikation erheblich eingeschränkt.

Das Kontinuumsmodell ist beschränkt auf die Wechselwirkung der Blasen mit dem äußeren Schallfeld durch die primäre Bjerknes-Kraft. Die Wechselwirkung der Blasen miteinander wird nicht berücksichtigt. 
(a)

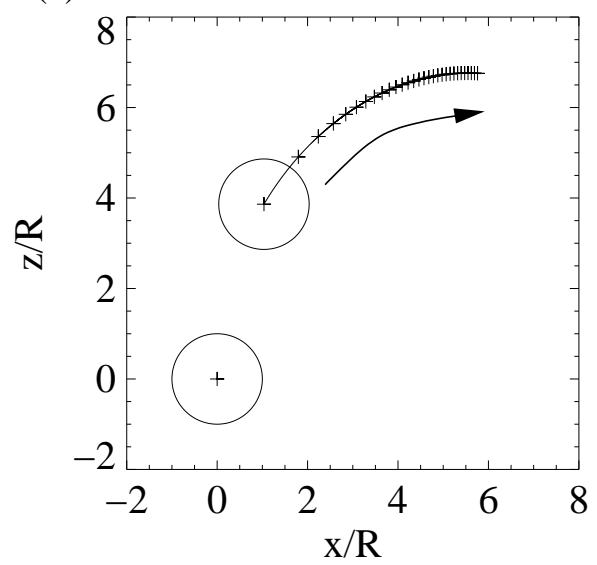

(c)

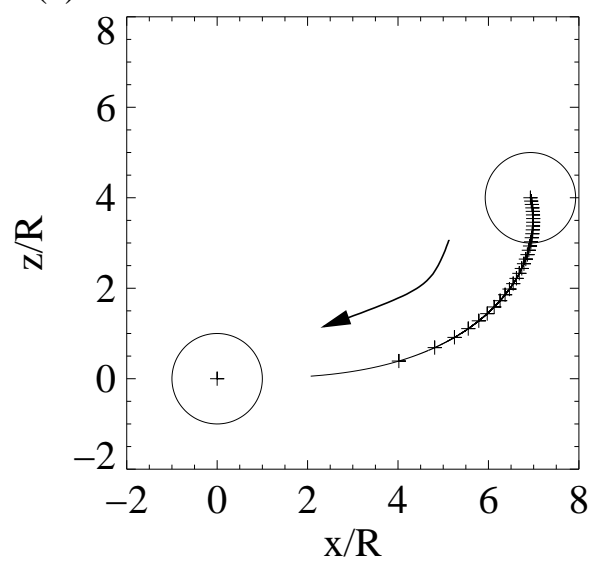

(b)

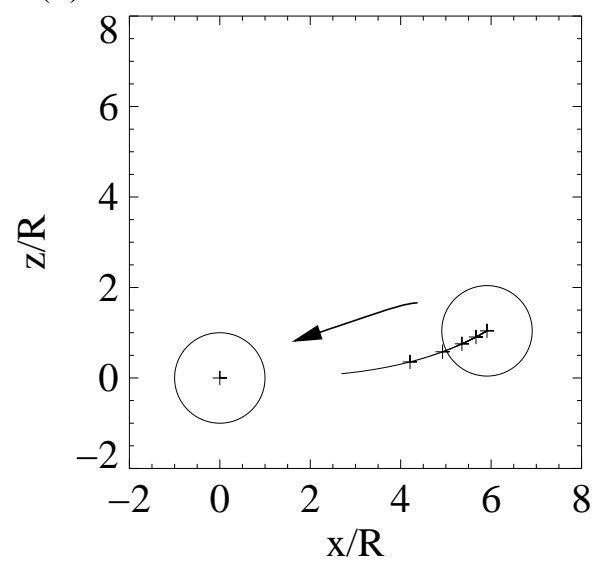

(d)

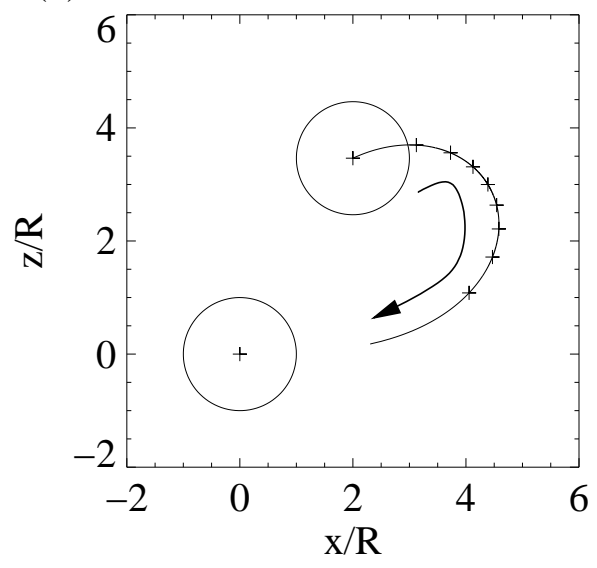

Abbildung 4.14.: Beispiele der ebenen Bewegung unter dem Einfluß der Dipol-DipolWechselwirkung. Die Anfangsbedingungen sind in den Relativkoordinaten (vgl. Abb. 4.13 [40]). (a) $\Theta=15^{\circ}, d=4 R_{0}$, (b) $\Theta=80^{\circ}, d=6 R_{0}$, (c) $\Theta=60^{\circ}, d=8 R_{0}$, (d) $\Theta=30^{\circ}, d=4 R_{0}$.

Das Partikelmodell beschreibt die Dynamik akustischer Kavitationsblasenfelder im Rahmen der Lagrangeschen Formulierung der inkompressiblen Hydrodynamik. Die Bewegungsgleichungen der Blasen können hierbei allein aus der kinetischen und potentiellen Energie des Systems abgeleitet werden. Die Wechselwirkung der Blasen miteinander kann als Multipolentwicklung in allgemeiner Form formuliert werden und entspricht einem effektiven Wechselwirkungspotential. Im Rahmen dieser Arbeit wurde die Wechselwirkung durch die Monopol-Monopol-, MonopolDipol- und Dipol-Dipol-Wechselwirkung beschrieben. Diese Formulierung erlaubt die Betrachung der Wechselwirkung durch Volumenoszillation und Translationsbewegung der Blasen. Es kann daher die Wechselwirkung mit einer äußeren makroskopischen Strömung betrachtet werden. Das Partikelmodell kann die nichtlineare Blasenoszillationen beschreiben: Damit besteht die Möglichkeit der Simulation mit experimentell relevanten Parametern.

Die grundlegende Annahme des Partikelmodells ist die Wirbelfreiheit der inkompressiblen Flüssigkeitsbewegung. Dies bedeutet eine Einschränkung, da die Wechselwirkung der Blasen 
durch ihre Nachlaufströmung nicht in allen Fällen vernachlässigt werden kann. Allerdings wurde in der vorliegenden Arbeit der Beschreibung durch ein einfaches Wechselwirkungmodell der Vorzug gegeben, um die wesentliche Dynamik zu verstehen.

Im Partikelmodell ist keine Rückwirkung auf das äußere Schallfeld berücksichtigt. Diese Rückwirkung ist bereits bei geringen Blasendichten wesentlich für die Dynamik des Systems. Für die Bewegung einzelner Blasen kann dieser Einfluß jedoch vernachlässigt werden. Es ist damit möglich, wesentliche Aussagen des Modells experimentell zu überprüfen.

Das Partikelmodell stellt ebenso wie das Kontinuumsmodell - und wie jedes Modell - eine Abstraktion des realen physikalischen Systems dar. Das Kontinuumsmodell beschreibt die makroskopische Dynamik gemittelter Größen auf langsamen Zeitskalen. Das Partikelmodell beschreibt hingegen die mikroskopische Oszillations- und Translationsbewegung der Blasen.

Zusammenfassend kann man folgendes feststellen: Das Kontinuumsmodell erlaubt, wegen der oben genannten Einschränkungen, keine Beschreibung der experimentellen Beobachtungen mit realistischen Parametern. Eine Beschreibung auf der Grundlage gemittelter, effektiver Eigenschaften setzt die Kenntnis der mikroskopischen Wechselwirkungen der Phasen voraus. Hierzu zählt das Verständnis der Kopplung von Translation und Oszillation der Blasen und der elementaren Blasen-Blasen-Wechselwirkungen. Es konnte gezeigt werden, daß die genannten Eigenschaften - insbesondere für die hier interessierenden nichtlinearen Blasenschwingungen - einen erheblichen quantitativen und qualitativen Unterschied zu der linearen Approximation aufweisen, welche die Grundlage des Kontinuumsmodells ist. Diese für das System essentiellen Eigenschaften können mit dem Kontinnumsmodell nicht beschrieben werden.

Das Partikelmodell erlaubt die detaillierte Untersuchung der nichtlinearen Wechselwirkung der Phasen und ermöglicht damit die Beschreibung des experimentellen Systems mit relevanten Parametern.

\subsection{Einfluß der Kompressibilität auf die Blasendynamik}

In diesem Abschnitt wird der Einfluß der Kompressibilität der Flüssigkeit auf die Dynamik der Blasen und ihre Kopplung untersucht. Wir beschränken uns hierbei im Folgenden auf die auf die durch die Kompressibilität verursachte endliche Laufzeit des Schalls, welche eine Zeitverzögerung der Kopplung der Blasen zur Folge hat. Für die Modellierung der Strukturbildung in akustischen Kavitationsblasenfelder ist insbesondere von Interesse, welche Änderungen sich für die sekundäre Bjerknes-Kraft ergeben.

Es wird daher zunächst ein nichtlineares Modell für zwei Blasenoszillatoren mit zeitverzögerter Kopplung formuliert [56]. Für kleine Anregungsamplituden können die Bewegungsgleichungen linearisiert und die Eigenschaften dieser linearen Lösung diskutiert werden. Für Anregungsdrücke, wie sie in experimentellen Aufbauten typisch sind, müssen die nichtlinearen Gleichungen numerisch integriert werden.

Modell der zeitverzögerten Kopplung In Abb. 4.15 (a) ist die einfache Geometrie des Modells illustriert. Wir betrachten zwei sphärische Blasen mit Ruheradien $R_{01}$ und $R_{02}$ die sich an den Orten $x_{1}$ und $x_{2}$ befinden. Der Abstand der Mittelpunkte der Blasen ist dann durch $L=\left|x_{1}-x_{1}\right|$ gegeben und wir nehmen an, daß er zeitlich konstant und so gewählt ist, daß $R_{1}(t), R_{2}(t) \ll L$ für alle Zeiten erfüllt bleibt.

Die radialen Oszillationen der Blasen und ihre zeitverzögerte Kopplung werden durch ein Sys- 
(a)

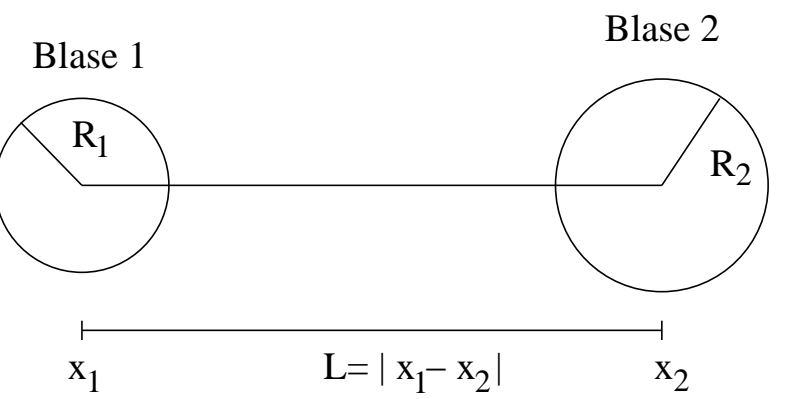

(b)
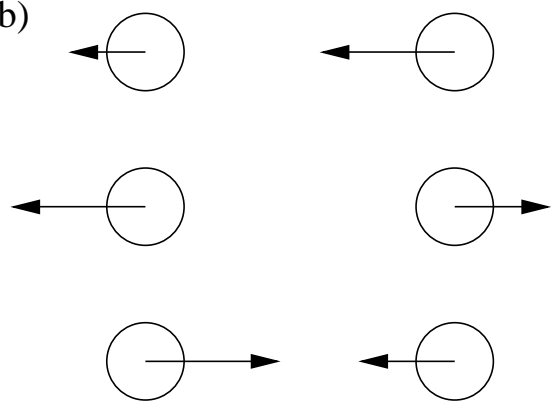

$\mathrm{F}_{12} \quad \mathrm{~F}_{21}$

Abbildung 4.15.: (a) Geometrie des Modells der zeitverzögerten Kopplung zweier Blasenoszillatoren. (b) Illustration der Asymmetrie der sekundären Bjerknes-Kraft als Folge der zeitverzögerten Kopplung.

tem modifizierter Keller-Miksis-Gleichung beschrieben [37]. Bezeichnet $c$ die endliche Schallgeschwindigkeit in der Flüssigkeit, so sind die Zeitverzögerungen der Kopplung durch die Laufzeiten

$$
\tau_{i}(t)=\left(L-R_{i}(t)\right) / c \quad \text { mit } \quad i=1,2
$$

gegeben. Um die numerische Behandlung des Problems zu vereinfachen, wird im folgenden davon ausgegangen, daß die Zeitabhängigkeit der Laufzeiten wegen $R_{1}(t), R_{2}(t) \ll L$ vernachlässigt werden kann. Mit der konstanten zeitverzögerten Kopplung $\tau$ sind die Bewegungsgleichungen des Systems durch

$$
\begin{array}{r}
\left(1-\frac{\dot{R}_{i}(t)}{c}\right) R_{i}(t) \ddot{R}_{i}(t)+\frac{3}{2} \dot{R}_{i}^{2}(t)\left(1-\frac{\dot{R}_{i}(t)}{3 c}\right)=\left(1+\frac{\dot{R}_{i}(t)}{c}\right) \frac{P_{i}}{\rho}+\frac{R_{i}(t)}{\rho c} \frac{d P_{i}}{d t} \\
-\frac{R_{j}(t-\tau)}{L}\left(R_{j}(t-\tau) \ddot{R}_{j}(t-\tau)+2 \dot{R}_{j}^{2}(t-\tau)\right), \quad i, j=1,2 .
\end{array}
$$

gegeben. Der Druck $P_{i}$ auf die Wand der $i$-ten Blase mit Gleichgewichtsradius $R_{0 i}$ ist

$$
P_{i}(t)=\left(P_{0}+\frac{2 \sigma}{R_{0 i}}\right)\left(\frac{R_{0 i}}{R_{i}(t)}\right)^{3 \gamma}-P_{0}-\frac{2 \sigma}{R_{i}(t)}-4 \eta \frac{\dot{R}_{i}(t)}{R_{i}(t)}-p_{a}(t)
$$

wobei $P_{0}$ den Umgebungsdruck, $\sigma$ und $\eta$ die Oberflächenspannung und die Viskosität der Flüssigkeit und $\gamma$ den Polytropenexponenten des Gases in der Blase bezeichnen. Es wird hier zunächst angenommen, daß der Anregungsdruck $p_{a}(t)=P_{a} \cos (\omega t)$ für beide Blasen gleich ist. Diese vereinfachende Annahme ist i.a. nicht erfüllt für große Abstände $L$, für die sowohl die Amplitude als auch die Phase der Anregung unterschiedlich sein können.

Um die Bedeutung der zeitverzögerten Kopplung für die Dynamik von Blasenfeldern zu untersuchen, betrachten wir nun im besonderen ihren Einfuß auf die Wechselwirkung der Blasen. Auf die gekoppelten Blasen wirkt eine sekundäre Bjerknes-Kraft, die durch

$$
\mathbf{F}_{i j}^{b}=-\frac{\rho}{4 \pi L^{2}}<\dot{V}_{i}(t-\tau) \dot{V}_{j}(t)>\hat{\mathbf{e}}_{i j} .
$$

gegeben ist. Diese Kraft wirkt von Blase $i$ auf Blase $j$, wobei $\hat{\mathbf{e}}_{i j}$ den Einheitsvektor von Blase $i$ zu Blase $j$, und $<.>$ das Zeitmittel über eine Periode der Anregung bezeichnet. Es kann als 
erstes Ergebnis festgestellt werden, daß die Kompressibilität der Flüssigkeit die Symmetrie der Wechselwirkung bricht, d.h. daß

$$
\mathbf{F}_{12}^{b} \neq \mathbf{F}_{21}^{b}
$$

gilt. Diese Asymmetrie ist möglich, sofern Impuls von dem Schallfeld mitgenommen wird. Die Kompressibilität der Flüssigkeit führt daher auf diese Weise zu einer zusätzlicher Dissipation. In Abb. 4.15 (b) ist diese Asymmetrie der sekundären Bjerknes-Kraft illustriert.

Linearisierung Für einen kleinen Anregungsdruck des externen Schallfeldes $\left(P_{a} \ll P_{0}\right)$ kann die Gl. (4.74) um die Gleichgewichtsradien $R_{i}(t)=R_{0 i}+r_{i}(t)$ mit $r_{i} \ll R_{0 i}$ linearisiert werden. Dies führt auf die lineare Bewegungsgleichung

$$
\ddot{r}_{i}(t)+\omega_{0 i}^{2} r_{i}(t)+f_{i} \dot{r}_{i}(t)+\frac{R_{0 j}^{2}}{L R_{0 i}} \ddot{r}_{j}(t-\tau)=-\frac{p_{a}(t)}{\rho R_{0 i}} .
$$

Es wird hierbei angenommen, daß die Gleichgewichtsradien die Bedingung $4 \eta / \rho c \ll R_{0 i} \ll$ $c / \omega=\lambda / 2 \pi$ erfüllen. Die linearen Resonanzfrequenzen und Dämpfungskoeffizienten sind gegeben durch

$$
\omega_{0 i}^{2}=\frac{1}{\rho R_{0 i}^{2}}\left(3 \gamma P_{0}+\frac{2 \sigma}{R_{0 i}}(3 \gamma-1)\right) \quad \text { und } \quad f_{i}=\frac{4 \eta}{\rho R_{0 i}^{2}}+\frac{\omega_{0 i}^{2} R_{0 i}}{c} .
$$

Der komplexe Ansatz $r_{i}(t)=A_{i} \exp (i \omega t)$ und $p_{a}(t)=P_{a} \exp (i \omega t)$ führt zu einer Lösung des linearen Systems in der Form

$$
\begin{aligned}
A_{i} & =-\frac{P_{a}}{D \rho R_{0 i}}\left(\left(\omega_{0 j}^{2}-\omega^{2}+i \omega f_{j}\right)+\frac{R_{0 j}}{L} \omega^{2} \exp (-i \omega \tau)\right) \\
D & =\left(\omega_{0 i}^{2}-\omega^{2}+i \omega f_{i}\right)\left(\omega_{0 j}^{2}-\omega^{2}+i \omega f_{j}\right)-\frac{R_{0 i} R_{0 i}}{L^{2}} \omega^{4} \exp (-2 i \omega \tau) .
\end{aligned}
$$

Für lineare Blasenschwingungen folgt dann mit Gl. (4.76) für die sekundäre Bjerkneskraft

$$
\mathbf{F}_{i j}^{b}=-\frac{2 \pi \rho \omega^{2}}{L^{2}} R_{10}^{2} R_{20}^{2}\left|A_{1}\right|\left|A_{2}\right| \cos \left(\phi_{1}-\phi_{2}-\omega \tau\right) \hat{\mathbf{e}}_{i j}
$$

mit der Phase $\phi_{i}=\arg \left(\operatorname{Im} A_{i} / \operatorname{Re} A_{i}\right)$. In Gl. (4.80) führt die zeitverzögerte Kopplung zu einem zusätzlichen Term

$$
\omega \tau=2 \pi \frac{L}{\lambda}
$$

der zusammen mit den Phasen $\phi_{1}$ und $\phi_{2}$ das Vorzeichen der Wechselwirkung bestimmt.

Ausgewählte Lösungen Die Ergebnisse der linearen Bewegungsgleichungen sind in Abb. 4.16 für eine Anregungsfrequenz von $\omega=2 \pi \times 20 \mathrm{kHz}$ als Funktion der Gleichgewichtsradien $R_{0 i}$ dargestellt. Die Ruheradien liegen im Intervall [ $5 \mu \mathrm{m} . . .15 \mu \mathrm{m}$ ]. Die Kopplung des linearen Gleichungssystems ist dann besonders stark, wenn beide Blasen einen Ruheradius nahe dem linearen Resonanzradius haben. Daher steigt die sekundäre Bjerknes-Kraft in Nähe der linearen Resonanzfrequenz deutlich an. Schwarz bedeutet eine repulsive, weiß eine attraktive Wechselwirkung. Die zeitverzögerte Kopplung führt in der Abb. 4.16 zu einem Verlust von Symmetrie. Diese Asymmetrie nimmt mit wachsendem $\tau$ stetig zu, bis bei einem Wert von $\tau=0.5 \cdot 2 \pi$ das Vorzeichenmuster invertiert ist. In Abb. 4.17 wird ein Beispiel für die sekundäre Bjerknes-Kraft zwischen zwei Blasen mit Ruheradien $R_{01}=R_{02}=5 \mu \mathrm{m}$ bei einer Anregungsfrequenz von 20 

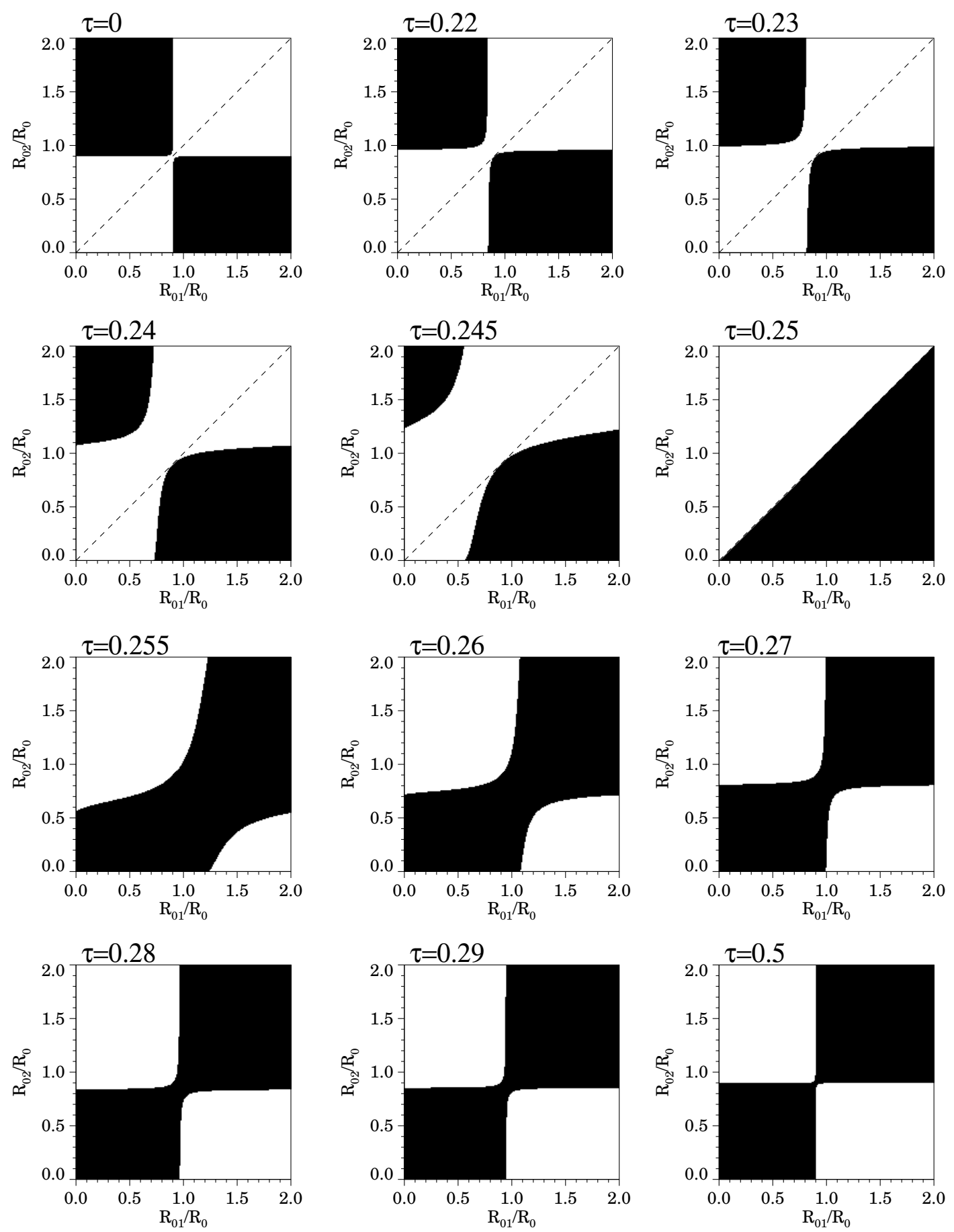

Abbildung 4.16.: Einfluß einer zeitverzögerten Kopplung auf die sekundäre Bjerknes-Kraft. Dargestellt ist das Vorzeichen der Wechselwirkung als Funktion der Zeitverzögerung $\tau$ in Einheiten von $2 \pi$. Schwarz bedeutet Abstoßung, weiß Anziehung. Die Vorzeichenstruktur ist periodisch in $\tau$ und ist für $\tau=0.5$ invertiert. Deutlich erkennbar ist der Verlust der Symmetrie der Wechselwirkung für $0<\tau<0.5$. 
(a)

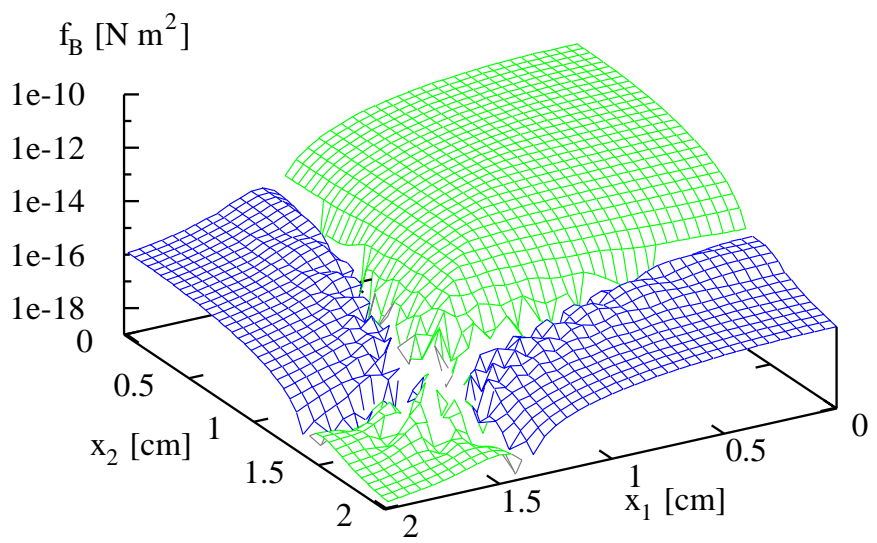

(b)

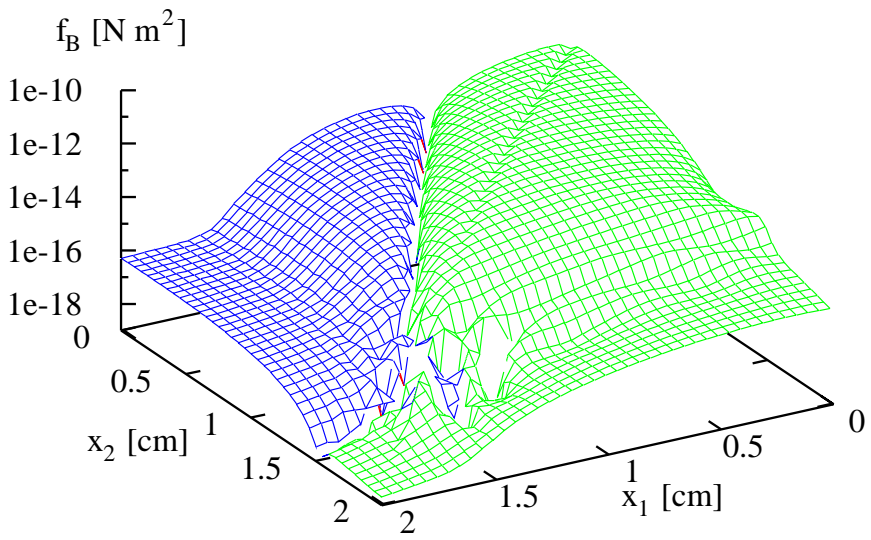

Abbildung 4.17.: Betrag des Koeffizienten der sekundären Bjerknes-Kraft zwischen zwei Blasen als Funktion des Abstandes für die Ruheradien $R_{10}=R_{20}=5 \mu \mathrm{m}$, die Anregungsfrequenz $f=20 \mathrm{kHz}$ und den Druck $p_{a}=150 \mathrm{kPa}$. In (a) ist der Koeffizient der sekundären Bjerknes-Kraft dargestellt, wie er sich für eine instantane Kopplung ergibt; die Figur ist symmetrisch. (b) Durch die zeitverzögerte Kopplung wird die Symmetrie der Wechselwirkung gebrochen. Die Farbe Grün bedeutet, daß Blase 2 von Blase 1 angezogen wird, blau bedeutet Abstoßung.

$\mathrm{kHz}$ und einem Druck $p_{a}=150 \mathrm{kPa}$ gezeigt. Dargestellt ist dort der durch $\left|\mathbf{F}_{i j}^{b}\right| \cdot L^{2}$ definierte Koeffizient der sekundären Bjerknes-Kraft als Funktion des Abstandes. In dieser Darstellung bedeutet eine grüne Farbcodierung, daß Blase 2 von Blase 1 angezogen wird und blau, daß Blase 2 abgestoßen wird. Bemerkenswert ist, daß bereits bei instantaner Kopplung in Abb. 4.17 (a) mit wachsendem Abstand ein Vorzeichenwechsel der sekundären Bjerknes-Kraft beobachtet werden kann. Dieser Effekt kann nicht im Rahmen der linearen Theorie erklärt werden. Abb.4.17 (b) zeigt den Symmetriebruch der nichtlinearen Wechselwirkung durch die zeitverzögerten Kopplung der Blasen.

\subsection{Zeitreihenanalyse}

Thema der folgenden Diskussion soll die Eigenschaft akustischer Kavitationsblasenfelder sein, auf verschieden Raum- und Zeitskalen Strukturen zu bilden [64]. Zur Charakterisierung der Komplexität des Systems wurden Methoden der nichtlinearen Dynamik und Zeitreihenanalyse 
angewandt $[48,31]$. Ein wesentliches Ergebnis dieser Arbeiten ist eine Dimensionsschätzung des Systems auf der Grundlage gemessener Zeitreihen von Hydrophonsignalen der akustischen Emission.

Am Beispiel eines periodisch akustisch angeregten Kavitationsblasenfeldes wird im folgenden demonstriert werden, daß aus einer niedrig-dimensionalen Delay-Rekonstruktion einer experimentellen Zeitreihe nicht notwendigerweise auf ein physikalisches System mit niedrigdimensionalem Attraktor geschlossen werden kann. Ursache für dieses allgemeine Phänomen ist die auch im chaotischen Fall vorliegende Phasenkohärenz zwischen den nichtlinearen Oszillatoren und dem treibenden Signal. Als Folge dieser Kohärenz fällt, wenn ein Ensemble von chaotischen Oszillatoren durch eine gemeinsame Anregung getrieben wird, die Kreuzkorrelation zweier nicht oder nur schwach gekoppelter chaotischer Oszillatoren nicht auf Null ab, sondern wird periodisch für große Zeitverschiebungen. Wenn sich dies ereignet, verschwindet die mittlere Antwort einer Population von $N$ Oszillatoren nicht, sondern wird periodisch für große $N$. Für mittlere Populationsgrößen erscheinen Attraktorrekonstruktionen mit Zeitverzögerungkoordinaten dieses gemittelten Signals als verrauscht periodisch, quasi-periodisch oder niedrigdimensional chaotisch. Die tatsächliche hohe Dimension des Signals ist verborgen in den kleinskaligen Oszillationen, die in der Regel experimentell nicht oder nur schwer zugänglich sind.

\subsubsection{Phasenkohärenz}

Zur Illustration des allgemeinen Phänomens betrachten wir zwei einfache Modellsysteme. Als Beispiel für ein autonomes System wählen wir die Lorenz-Gleichungen in der üblichen Form

$$
\begin{aligned}
\dot{x} & =10(y-x), \\
\dot{y} & =x(28-z)-y, \\
\dot{z} & =x y-\frac{8}{3} z \quad \text { mit } \quad(x(0), y(0), z(0))=\left(x_{0}, y_{0}, z_{0}\right) .
\end{aligned}
$$

Als zweites Modellsystem betrachten wir ein nicht-autonomes System von $N$ DuffingOszillatoren, welches gegeben ist durch

$$
\ddot{x}_{i}+d \dot{x}_{i}+b_{i} x_{i}+x_{i}^{3}=f(t) \quad \text { mit } \quad i=1, \ldots, N
$$

und dem Parametern für Dämpfung $d=0.2$, der Amplitude $a=40$ und der Frequenz $\omega=1$. Die Koeffizienten $b_{i}$ sind gleichverteilt im Intervall $[0.9,1.1]$ gewählt. Die Oszillatoren in Gl. (4.82) werden getrieben und über ein Summensignal - im Sinne eines mean field - gekoppelt durch

$$
f(t)=a \cos (\omega t)+c s(t) \quad \text { mit } \quad s(t)=\frac{1}{N} \sum_{i=1}^{N} x_{i}(t) .
$$

Die Konstante $c$ charakterisiert die Kopplungsstärke. Abb. 4.18 zeigt die Zeitentwicklung von Lorenz- und Duffing-Systemen. Für die Lorenz-Gleichungen werden zwei Trajektorien dargestellt mit ähnlichen, aber nicht identischen Anfangsbedingungen. Die sensitive Abhängigkeit von den Anfangsbedingungen führt nach kurzer Zeit zu einem Auseinanderlaufen der Lösungen. Dieses Verhalten spiegelt sich im Zeitverlauf des Korrelationskoeffizienten wider. Nur in der Anfangsphase ist er nahe eins und fällt dann rasch gegen null. Auch für die Duffing-Oszillatoren werden zwei ähnliche Anfangsbedingungen gewählt. Aber anders als beim autonomen LorenzSystem prägt hier die äußere Anregung der Lösung eine nahezu periodische Struktur mit der durch die Periode der Anregung gegebenen Zeitskala. Lediglich auf kleineren Zeit- und Amplitudenskalen zeigen die beiden Lösungen der Duffing-Oszillatoren deutliche Unterschiede. Im 
(a)

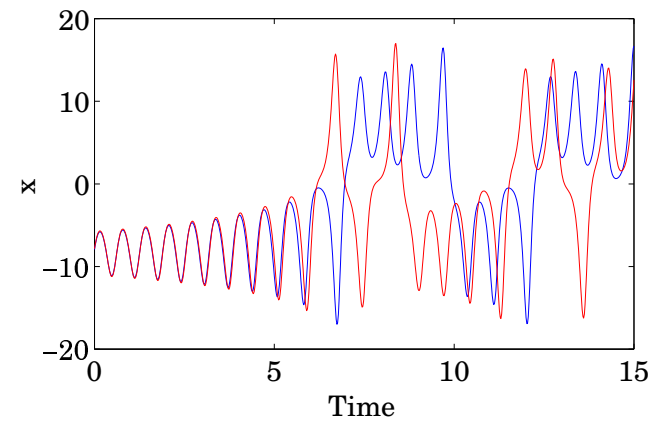

(c)

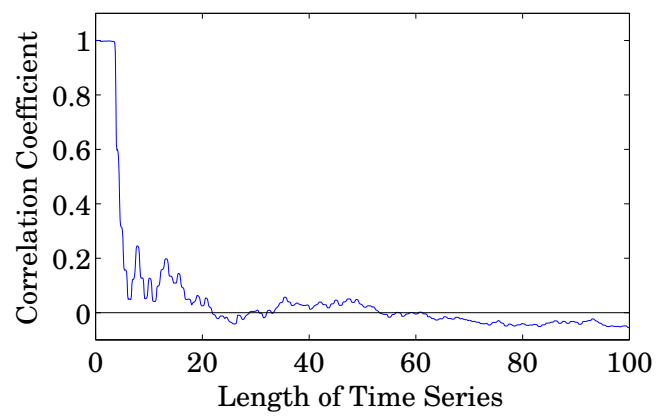

(b)

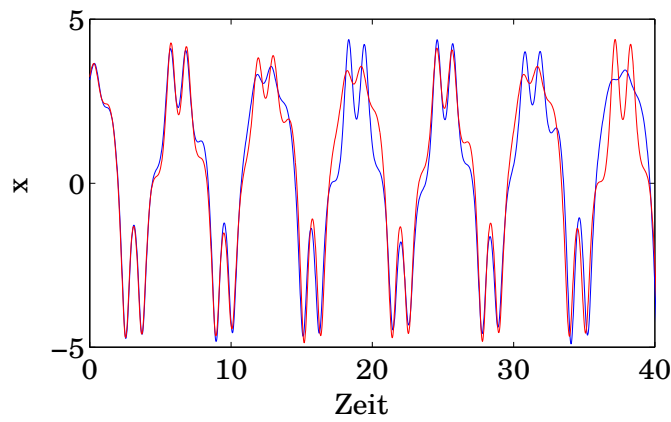

(d)

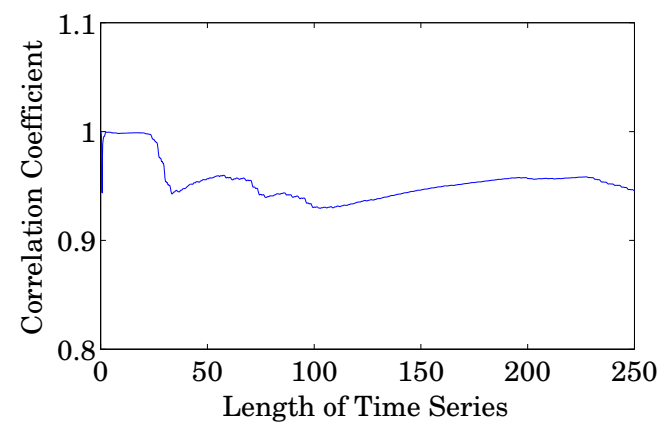

Abbildung 4.18.: Zerfall der Korrelationen am Beispiel des autonomen Lorenz- und des getriebenen Duffing-Systems. (a) Zwei Lösungen der Lorenz-Gleichungen mit ähnlichen, aber nicht identischen Anfangsbedingungen, die nach kurzer Zeit auseinanderlaufen. (b) Zwei Lösungen des Duffing-Oszillators, die ebenfalls mit ähnlichen, aber nicht identischen Lösungen starten. (c) Der Korrelationkoeffizient für die Lösungen der Lorenz-Gleichungen fällt rasch ab. (d) Der Korrelationskoeffizient für die Lösungen der Duffing-Oszillatoren sinkt durch die externe Anregung in diesem Beispiel nicht unter 0.9.

Gegensatz zu dem autonomen Lorenz-System sinkt bei dem getriebenen Duffing-System der Korrelationskoeffizient in diesem Beispiel nicht unter 0.9. Beide Lösungen bleiben kohärent bzw. korreliert. Dies steht in Zusammenhang mit dem allgemeinen Phänomen der Phasensynchronisation.

Die Auswirkungen dieser Eigenschaft auf die Dimensionsanalyse werden an einer Attraktorrekonstruktion des Summensignals Gl. (4.83) betrachtet. Für die Verzögerungskoordinaten gilt

$$
y(t)=(s(t), s(t-\tau), s(t-2 \tau))
$$

mit $\tau=2 \pi /(5 \omega)[36,1]$. Ein Beispiel für eine solche Attraktorrekonstruktion ist in Abb. 4.19 (a) für einen einzelnen Duffing-Oszillator dargestellt. Abb. 4.19 (b) zeigt die Attraktorrekonstruktion des Summensignals eines Systems von $N=100$ ungekoppelten Duffing-Oszillatoren $(c=0)$. Die differenzierte Detailstruktur der Attraktorrekonstruktion des Einzeloszillators ist hier durch die Mittelung bei der Bildung des Summensignals fast vollständig verschwunden. 
(a)

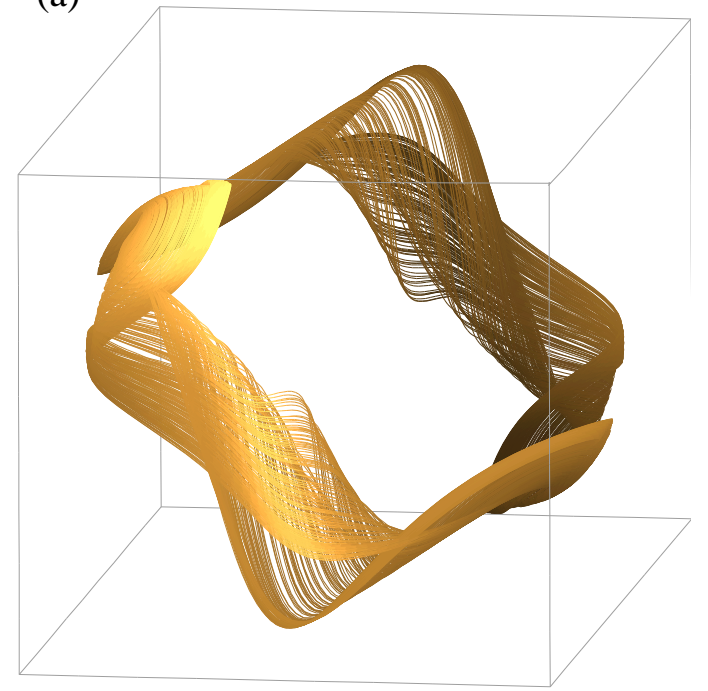

(b)

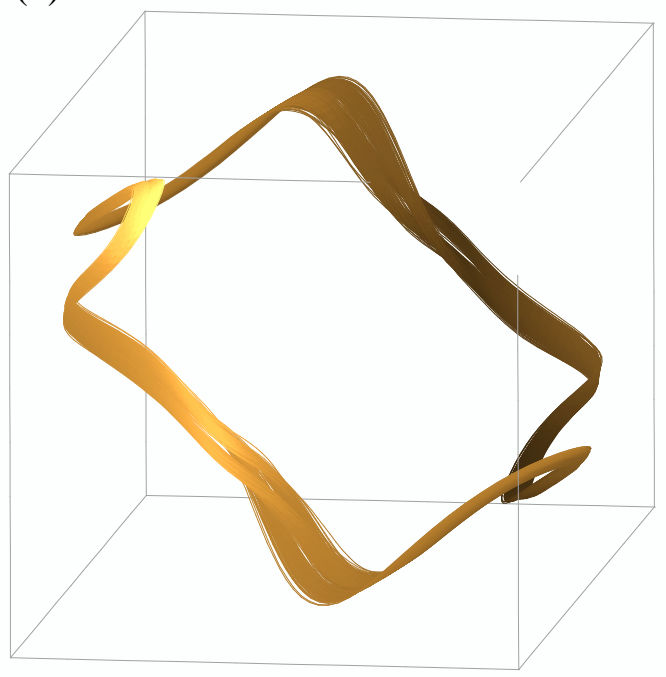

Abbildung 4.19.: (a) Attraktorrekonstruktion eines Duffing-Oszillators. (b) Attraktorrekonstruktion des Summensignals Gl. (4.83) für ein Systems von $N=100$ ungekoppelten Oszillatoren (Kopplungsstärke $c=0$ ).

Der Einfluß der Kopplung wird nun mit Hilfe eines Poincaré-Schnittes betrachtet, der durch

$$
(x(t), x(t+T)) \quad \text { mit } \quad T=2 \pi / \omega
$$

definiert wird. In Abb. 4.20 sind Poincaré-Schnitte sowohl für einen Einzeloszillator als auch für ein System von $N=100$ gekoppelten Duffing-Oszillatoren $(c=10)$ dargestellt. Während der Poincaré-Schnitt des einzelnen Oszillators die differenzierte Faltung des Attraktors widerspiegelt, zeigt der Poincaré-Schnitt des Summensignals der einhundert Oszillatoren offenbar wenig Struktur.

In Abb. 4.21 ist das Ergebnis der Dimensionsanalyse eines Systems von $N=100$ gekoppelten Duffing-Oszillatoren $(c=10)$ dargestellt. Die Korrelationsdimension kann für mittlere und größere Skalen zu $\mathrm{D}_{2}=2.1$ abgeschätzt werden. Für kleine Skalen hingegen wächst diese deutlich an. Im betrachteten Beispiel findet man hier als Schätzung der Dimension etwa $\mathrm{D}_{2}=7.1$. Hierbei handelt es sich aber um eine untere Schranke, da für wachsende Dimension erheblich mehr Datenpunkte notwendig sind [68].

Die tatsächliche hohe Dimension des Systems ist in diesem Beispiel in kleinskaligen Oszillationen verborgen.

\subsubsection{Modell des Kavitationsblasenfeldes}

Das beobachtete Phänomen der verallgemeinerten Phasenkohärenz getriebener Oszillatoren läßt sich auf die akustischen Eigenschaften von Kavitationsblasenfeldern anwenden. Zur Illustration werden die Ergebnisse der numerischer Simulationen einer Blasenwolke mit experimentell gemessenen Zeitreihen verglichen [52]. In der numerischen Simulation betrachten wir ein System von $\mathrm{N}$ sphärischen Blasen mit einer vorgegebenen Verteilung der Ruheradien $R_{0 i}$ und Positionen $\mathbf{x}_{i}$. Die Blasen werden von einem äußeren Schallfeld getrieben. Wenn die Drift der Blasenschwerpunkte vernachlässigt werden kann, so sind die radialen Oszillationen der Blasen durch 
(a)

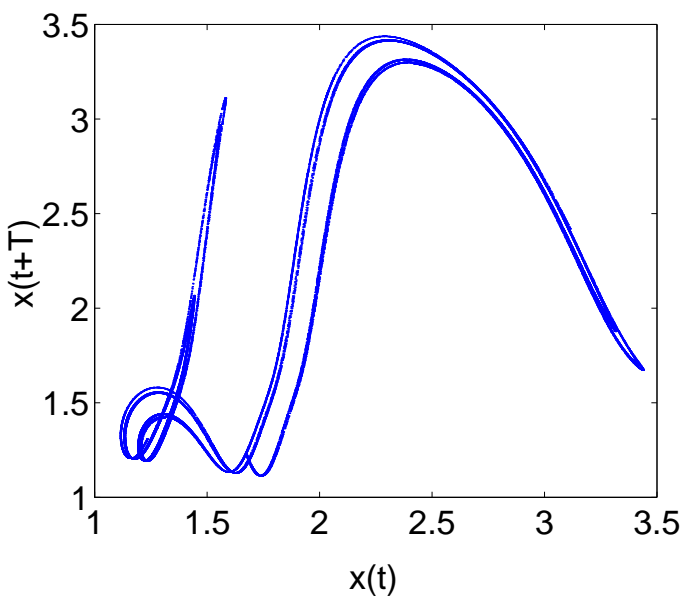

(b)

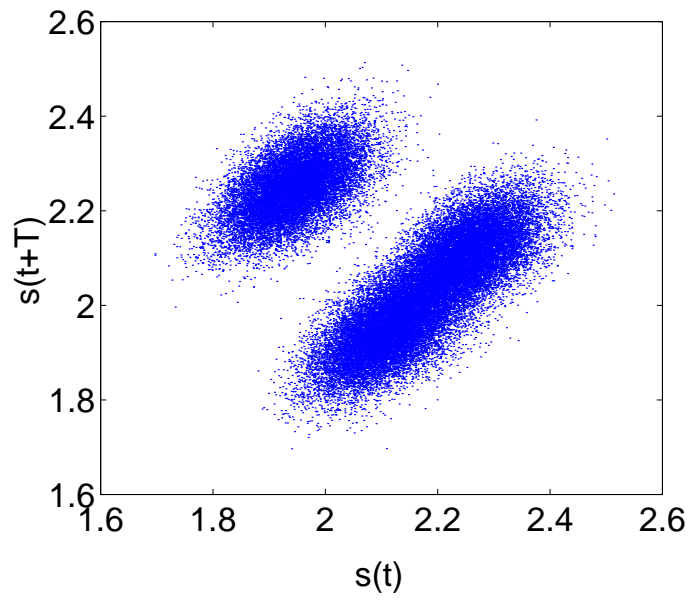

Abbildung 4.20.: (a) Poincaré-Schnitt eines Duffing-Oszillators. (b) Poincaré-Schnitt des Summensignals Gl. (4.83) für ein System von $N=100$ gekoppelten Oszillatoren (Kopplungsstärke $c=10$ ). Dargestellt sind jeweils 50000 Datenpunkte.

eine modifizierte Keller-Miksis-Gleichung ${ }^{4}$ (4.86) in der Form

$$
\begin{aligned}
\left(1-\frac{\dot{R}_{i}}{c}\right) R_{i} \ddot{R}_{i} & +\frac{3}{2} \dot{R}_{i}^{2}\left(1-\frac{\dot{R}_{i}}{3 c}\right)+\sum_{j \neq i}^{N} \frac{p_{i j}}{\rho}=\left(1+\frac{\dot{R}_{i}}{c}\right) \frac{p_{l}}{\rho}+\frac{R_{i}}{\rho c} \frac{d p_{l}}{d t}, \\
p_{l} & =\left(p_{0}+\frac{2 \sigma}{R_{0 i}}\right)\left(\frac{R_{0 i}}{R_{i}}\right)^{3 \kappa}-p_{0}-\frac{2 \sigma}{R_{i}}-\frac{4 \mu}{R_{i}} \dot{R}_{i}-p_{a}, \\
p_{a} & =p_{a}\left(t, \mathbf{x}_{i}\right)=P_{a}\left(\mathbf{x}_{i}\right) \cos (\omega t), \quad i=1, \ldots, N
\end{aligned}
$$

gegeben, wobei der Punkt die Zeitableitung bezeichnet. Für Luftblasen in Wassser bei $20^{\circ} \mathrm{C}$ mit einem Polytropenexponenten $\kappa=1.4$ werden die üblichen Parameter für die Oberflächenspannung $\sigma$, die Flüssigkeitsdichte $\rho$, die Viskosität $\mu$, den Umgebungsdruck $p_{0}$ und die Schallgeschwindigkeit in der Flüssigkeit $c$ verwendet. Es wird die Anregungsfrequenz $\omega=2 \pi \cdot 21 \mathrm{kHz}$ und der maximale Anregungsdruck $P_{a}=150 \mathrm{kPa}$ gewählt. In Gl. (4.86) bezeichnet $p_{l}$ den Druck der Flüssigkeit an der Blasenoberfläche. Die numerischen Simulationen wurden durchgeführt mit $N=300$ Blasen, deren Gleichgewichtsradien $R_{0 i}$ gleichverteilt im Intervall $[5 \mu \mathrm{m} \ldots 30 \mu \mathrm{m}]$ und deren Orte $\mathbf{x}_{k}$ gaussverteilt mit Varianz $\sigma_{n}=1 \mathrm{~cm}$ gewählt wurden. Sofern für die Blasenverteilung $\sigma_{n} \ll \lambda$ gilt, kann vereinfachend angenommen werden, daß alle Blasen die gleiche Anregung erfahren. Es wird ferner angenommen, daß die Bewegung der Flüssigkeit in der Nähe einer Blase sphärisch symmetrisch ist. Dann kann in der Umgebung der oszillierenden Blase die

${ }^{4}$ Ein implizites Differentialgleichungssystem zweiter Ordnung $C(x, y) \ddot{y}=f(x, y, \dot{y})$ kann durch die neuen Variablen $z=\dot{y}$ und $u=\dot{z}=\ddot{y}$ in die differential-algebraische Gestalt

$$
\begin{aligned}
\dot{y} & =z \\
\dot{z} & =u \\
0 & =C(x, y) u-f(x, y, z)
\end{aligned}
$$

gebracht werden. 
(a)

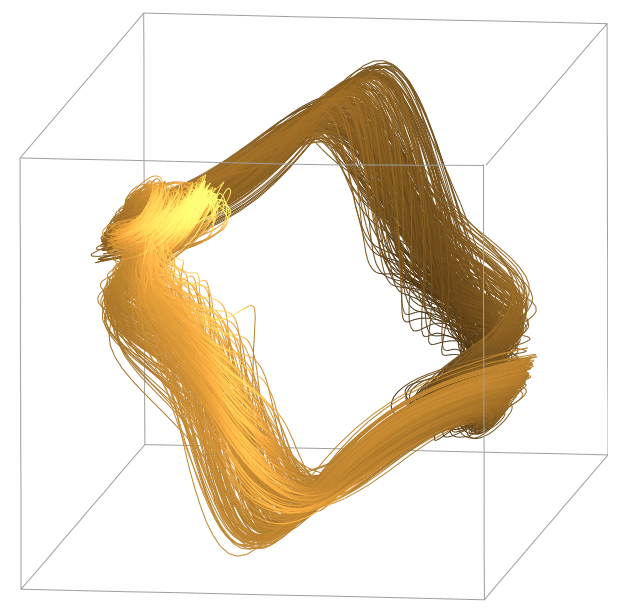

(b)

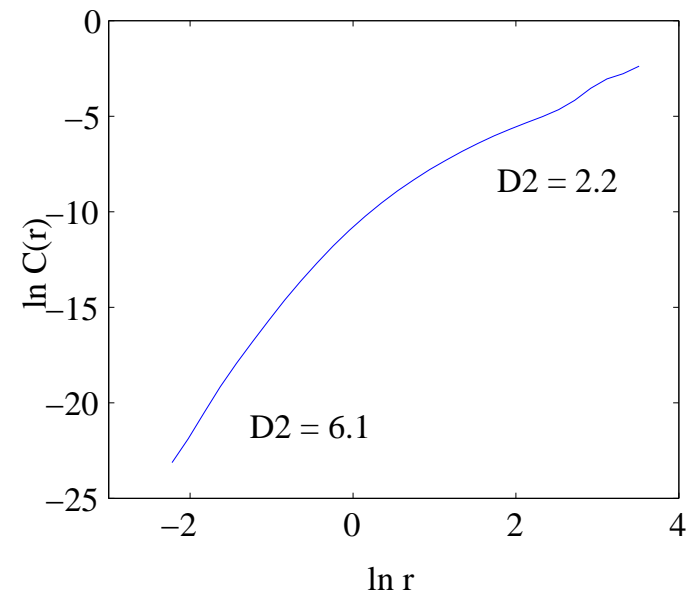

Abbildung 4.21.: (a) Attraktorrekonstruktion des Summensignals Gl. (4.83) von $N=100$ gekoppelten Duffingoszillatoren (Kopplungsstärke $c=10$ ). (b) Dimensionsanalyse Korrelationsdimension.

Flüssigkeit als inkompressibel angenommen werden und das Geschwindigkeitsfeld in der Form

$$
w=\frac{R_{i}^{2} \dot{R}_{i}}{r_{i}^{2}}
$$

geschrieben werden. Hierbei bezeichnet $r_{i}$ die radiale Koordinate eines lokalen Koordinatensystems, dessen Ursprung im Schwerpunkt der Blase liegt. Um den Druck zu berechnen, wird die Bewegungsgleichung der Flüssigkeit

$$
\rho \frac{\partial w}{\partial t}+\frac{\partial p_{i}}{\partial r_{i}}=0
$$

verwendet, wobei mit $p_{i}$ der von der $i$-ten Blase emittierte Druck bezeichnet wird. Der nichtlineare Konvektionsterm $w \partial w / \partial r_{k}$ ist in Gl. (4.88) vernachlässigt worden, da er von Ordnung $r_{i}^{-5}$ und daher klein gegenüber dem ersten Term ist. Setzen wir Gl. (4.87) in Gl. (4.88) ein und führen die Integration durch, so erhalten wir folgenden Ausdruck für den Druck:

$$
p_{i}=\frac{\rho}{r_{i}} \frac{d}{d t}\left(R_{i}^{2} \dot{R}_{i}\right)=\frac{\rho}{r_{i}}\left(R_{i}^{2} \ddot{R}_{i}+2 R_{i} \dot{R}_{i}^{2}\right) .
$$

Es sei angemerkt, daß Gl. (4.89) nur in der Nähe der Blase gültig ist, d.h. wenn $r_{i}<c / \omega$ gilt. Es ist leicht zu zeigen, daß für größere Abstände der Druck durch die Gleichung

$$
p_{i}=\left.\frac{\rho}{r_{i}} \frac{d}{d t}\left(R_{i}^{2} \dot{R}_{i}\right)\right|_{t-r_{i} / c}
$$

beschrieben wird. 
Im Experiment wird die Schallemission des Blasenfeldes mit einem Hydrophon gemessen. In Analogie hierzu wird in der numerischen Simulation der Druck $P_{h}$ am Aufpunkt $\mathbf{x}_{h}$ des Hydrophons bestimmt. Dieser Druck ist durch die Superposition des Anregungsdruckes mit der Summe der Beiträge aller Einzelblasen gegeben, d.h.

$$
P_{h}(t)=p_{a}\left(t, \mathbf{x}_{h}\right)+\sum_{k=1}^{N} p_{k}(t) .
$$

Die Übertragungseigenschaften des Hydrophons werden durch ein Tiefpassfilter

$$
\ddot{u}+\gamma \omega_{0} \dot{u}+\omega_{0}^{2} u=P_{h}(t) \quad \text { mit } \quad \omega_{0}=2 \pi f_{0}
$$

mit der Abschneidefrequenz $f_{0}=400 \mathrm{kHz}$ und der Dämpfung $\gamma$ nachgebildet. Abb. 4.22 zeigt eine dreidimensionale Attraktorrekonstruktion mit der Delayzeit $\tau$,

$$
\mathbf{u}(t)=(u(t), u(t+\tau), u(t+2 \tau)),
$$

des experimentell gemessenen Hydrophonsignals und der numerischen Simulation $P_{h}(t)$. Beide Rekonstruktionen ähneln einem verrauschten niedrig-dimensionalen Attraktor. Dennoch ist zumindest das simulierte Hydrophonsignal sehr hochdimensional, da sich in diesem Beispiel mehr als $70 \%$ der $N=300$ Blasen chaotisch verhalten. Um diese hohe Dimensionalität der Zeitreihe des Hydrophonsignals aufzulösen, ist eine hinreichend große Datenmenge von sehr guter Qualität notwendig.

In der bisherigen Diskussion wurde angenommen, daß das betrachtete System stationär ist. Für das in den Gln. (4.82) und (4.83) betrachtete System gekoppelter Duffing-Oszillatoren bedeutet dies, daß die das Ensemble charakterisierenden Parameter und insbesondere die Stärke der Kopplung zeitlich konstant sind. In einem experimentellen System sind diese Parameter jedoch oft zufälligen Fluktuationen oder systematischen Änderungen unterworfen.

Die Dynamik eines akustischen Kavitationsblasenfeldes wird bestimmt durch die räumliche Anordnung der Blasen und die Verteilung der Ruheradien. Die Verteilung der Blasen im Raum bestimmt ihr Schwingungsverhalten sowohl durch die Ortsabhängigkeit des Anregungsdrucks als auch durch die vom gegenseitigen Abstand abhängige Amplitude und Phase der Kopplung. In dem experimentell beobachteten Kavitationsblasenfeld bewegen sich die Blasen unter dem Einfluß äußerer Kräfte und wechselseitiger Kopplung und verändern hierdurch ihre geometrische Anordnung im Raum. Durch diffusives Wachstum, aber auch durch Kollision und Fragmentierung ändern sich die Ruheradien der Blasen. Daher ist die Änderung des Blasenfeldes charakterisiert durch die typischen Zeitkonstanten für die Drift der Blasen und diffusives Wachstum sowie durch ihre mittlere Lebensdauer. Zur Illustration sind in Abb. 4.23 (a,c) zwei im Abstand von einer Sekunde aufgenommene Momentaufnahmen eines akustischen Kavitationsblasenfeldes dargestellt. Die Struktur der Filamente und die Anordnung der Blasen in ihnen hat sich sichtbar verändert. Die zu diesen räumlichen Konfigurationen korrespondierenden Attraktorrekonstruktionen des experimentell gemessenen Hydrophonsignals sind in den Abb. 4.23 (b,d) gezeigt. Abgesehen von einer strukturellen Ähnlichkeit unterscheiden sich beide Darstellungen deutlich voneinander.

Zusammmenfassend erkennt man, daß Delay-Rekonstruktionen von Zeitreihen, die durch hochdimensionale dynamische Systeme erzeugt worden sind, Objekte ("Attraktoren") liefern, die niedrig-dimensional auf experimentell zugänglichen mittleren und großen Skalen erscheinen. Eine Aussage über die tatsächliche Komplexität des experimentellen Systems erfordert daher notwendigerweise die detaillierte Betrachtung der involvierten Raum- und Zeitskalen. Dieser 

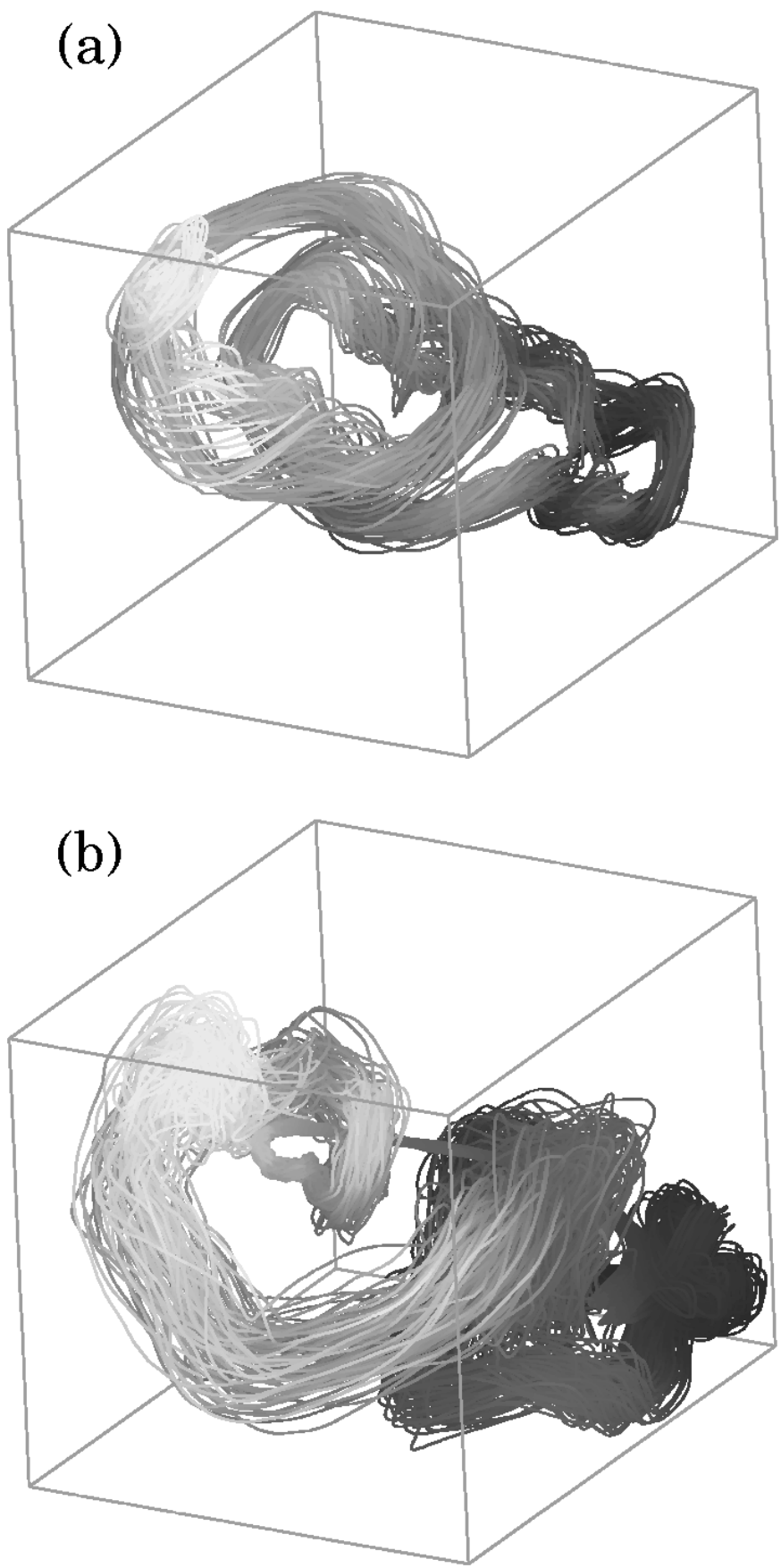

Abbildung 4.22.: (a) Typische drei-dimensionale Attraktorrekonstruktion eines experimentellen Hydrophonsignals. (b) Attraktorrekonstruktion eines numerisch simulierten Signals, die der des experimentellen Signals in (a) qualitativ ähnelt. 
(a)

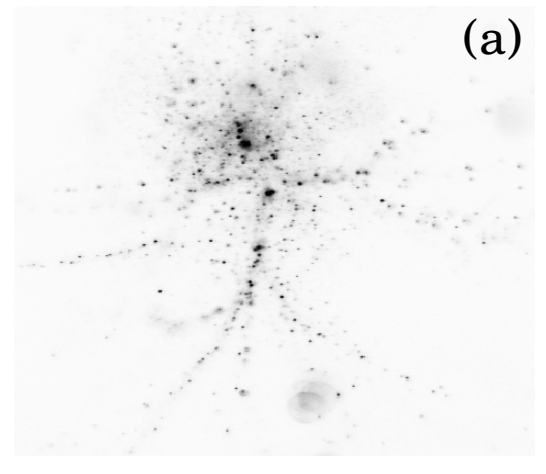

(c)

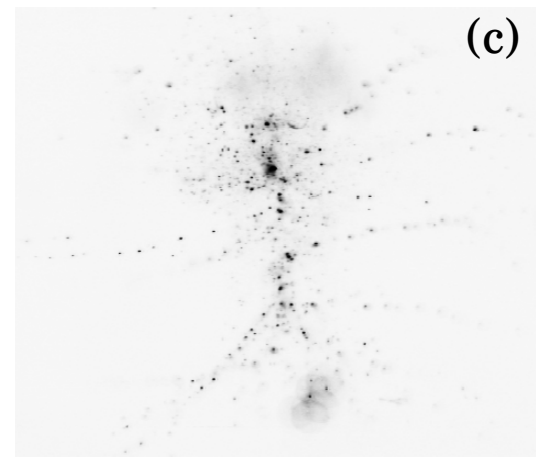

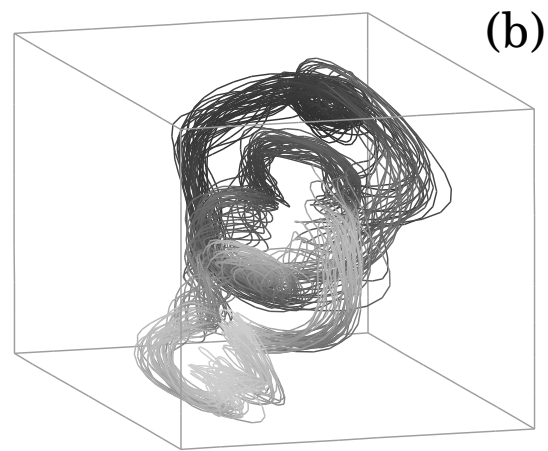

(b)

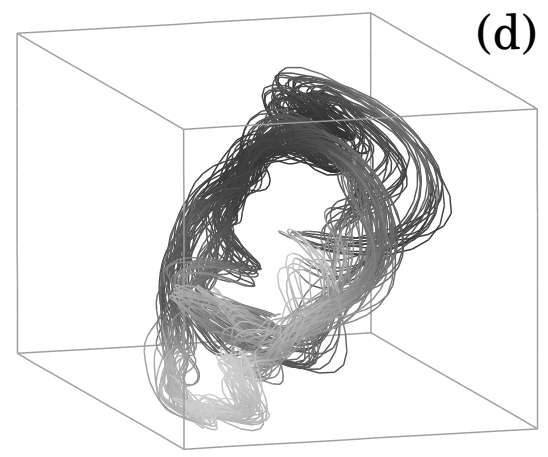

Abbildung 4.23.: (a) und (c) zeigen Momentaufnahmen der räumlichen Struktur des Kavitationsblasenfeldes in einem Abstand von etwa einer Sekunde. Die Grauwertverteilung der Bilder wurden invertiert um eine bessere Sichtbarkeit der Blasen zu erzielen. (b) und (d) zeigen die korrespondierenden dreidimensionalen Attraktorekonstruktionen des experimentellen Hydrophonsignals.

Effekt ist zu beobachten in großen Systemen getriebener (chaotischer) Oszillatoren mit hochdimensionalen Attraktoren im Zustandsraum. Die scheinbar niedrige Dimension kann zu Fehlinterpretationen führen, insbesondere hinsichtlich der Möglichkeit der Steuerung und Kontrolle dieses Oszillatorsystems. Um dieses Phänomen zu demonstrieren, wurde ein System von $N=300$ durch ein Schallfeld getriebener Blasen simuliert. Ein Vergleich mit anderen Oszillatorsystemen zeigt, daß der grundlegende Mechanismus, d.h. die Phasenbeziehung zwischen periodischer Anregung und chaotischen Oszillatoren, ein allgemeines Phänomen ist.

\subsection{Zusammenfassung}

In diesem Kapitel wurden zwei Modelle zur Beschreibung akustischer Kavitationsblasenfelder vorgestellt und diskutiert. Grundlegend für die Modellierung war die Analyse der in dem experimentellen System beobachteten Raum- und Zeitskalen. Ausgehend von dieser Diskussion können die Modelle in Klassen eingeteilt werden. Ein Kontinuummodell beschreibt die makroskopischen Eigenschaften gemittelter Größen auf langsamen Zeitskalen. Der Mittelungsprozeß führt naturgemäß zu einem Verlust von Information auf mittleren und kleinen Skalen. Insbesondere auf diesen mikroskopischen und schnellen Skalen liefert das vorgestellte Partikel-Modell Aufschluß über die Dynamik der Einzelblase und ihre Wechselwirkung mit anderen Blasen. Als Wechselwirkungen wurden im einzelnen die Monopol-Monopol, Dipol-Dipol- und Monopol- 
Dipol-Wechselwirkung diskutiert.

Ein grundlegendes Phänomen von Basenfeldern ist der Impulstransport. Hier liefert die Diskussion des Tensors der zusätzlichen Masse die Möglichkeit, einen Stabilitätsbegriff für die Bewegung der Blasen zu definieren. Beide Modelle - das Kontinuummodell ebenso wie das Partikel-Modell - erlauben nicht die Modellierung der direkten Wechselwirkung der Blasen durch Kollision und Fragmentierung, wie sie im Experiment beobachtet werden. Weiterhin bleibt die Rückwirkung der Änderung der Blasenverteilung auf das anregende Schallfeld unberücksichtigt. Die Hamiltonsche bzw. Lagrangesche Formulierung behandelt das Fluid als inkompressibel. Die Berücksichtigung der Kompressiblität kann zu einer signifikanten Änderung des Charakters der Blasen-Blasen-Wechselwirkung fuhren. Dies wurde an dem Beispiel eines Systems zeitverzögert gekoppelter Blasenoszillatoren demonstriert.

Zum Abschluß dieses Kapitels wurde ein zentraler Aspekt der Arbeit vertieft. Anknüpfend an die frühen Arbeiten über die Dimensionsanalyse akustischer Kavitationsblasenfelder konnte gezeigt werden, daß dieses System auf mikroskopischen Raum- und Zeitskalen eine hochdimensionale Dynamik zeigt. 


\section{Zusammenfassung}

In dieser Arbeit wurden akustische Kavitationsblasenfelder experimentell und theoretisch untersucht. Ausgangspunkt war das in Kapitel 1 vorgestellte grundlegende Schema des funktionalen Zusammenspiels der das System bildenden Komponenten. Für die Charakterisierung der raumzeitlichen Strukturen, d.h. der Anordnung der Blasen und ihrer Beziehung zueinander, ist die Analyse der Raum- und Zeitskalen notwendig. Die zentrale Zielsetzung dieser Arbeit war, das Verständnis der Wechselwirkung der Blasen auf mikroskopischer Skala zu vertiefen und ihre Bedeutung für das Entstehen und die Stabilität makroskopischer Strukturen zu untersuchen.

In Kapitel 2 wurden die hierfür entwickelten und verwendeten experimentellen Methoden eingeführt. Ziel der Messungen war die Bestimmung der Positionen, Geschwindigkeiten und Radien der Blasen. Es wurden die hierzu entwickelten und implementierten Bildbearbeitungsmethoden vorgestellt und deren Fehlerquellen abgeschätzt. Von besonderem Interesse für das Verständnis des physkalischen Systems und als Grundlage für eine folgende theoretische Beschreibung ist die Kenntnis der Trajektorien der Blasen. Daher wurde als wesentliche experimentelle Methode ein Verfahren zur Particle Tracking Velocimetry implementiert. Im Gegensatz zu den typischen Anwendungen mit Tracer-Partikeln, die der Strömung passiv folgen, nehmen die Kavitationsblasen aktiv am Geschehen teil. Kollisions- und Fragmentationsereignisse erfordern daher eine aufwendige Formulierung der Algorithmen zur Verfolgung der Blasen über eine Bildsequenz. Diese wurden im Rahmen dieser Arbeit entwickelt und erfolgreich angewandt. Desweiteren wurde ein Verfahren zur optischen Radienmessung entwickelt.

In Kapitel 3 wurden die Ergebnisse der experimentellen Untersuchung vorgestellt und diskutiert. Mit der im Meßaufbau verwendeten Hochgeschwindigkeits-Kamera konnten die Translationsbewegung der Blasen verfolgt und ihre Trajektorien durch den Tracking-Algorithmus bestimmt werden. Die experimentellen Untersuchungen in dieser Arbeit haben gezeigt, daß Kollisionsereignisse eine wesentliche Rolle für das Verständnis der Dynamik akustischer Kavitationsblasenfelder spielen. Diese Prozesse der direkten Blasen-Blasen-Wechselwirkung sind in der theoretischen Beschreibung in der Literatur bisher nicht der experimentellen Beobachtung entsprechend berücksichtigt worden. Dies beruht im Wesentlichen auf den erheblichen methodischen und algorithmischen Schwierigkeiten, die eine theoretische und numerische Beschreibung eines Mehrphasensystems aufwirft. Fortschritte in der direkten numerischen Simulation dieser für die Dynamik elementaren Wechselwirkungsprozesse geben Anlaß zu Hoffnung. Ungeachtet dieser Schwierigkeiten sollten in anschließenden Arbeiten diese Phänomene detailliert mit Hilfe der Ultra-Hochgeschwindigkeits-Photographie experimentell untersucht werden.

Die bisher in der Literatur vorgestellten experimentellen Untersuchungen beschreiben die Entstehung und die Dynamik filamentartiger Strukturen in akustischen Kavitationsblasenfeldern. Im Rahmen dieser Arbeit konnte darüber hinausgehend der Übergang dieser dendritischen Strukturen zu lokalisierten, kompakten Anordnungen von Blasen oder Blasenfragmenten experimentell beobachtet und detailliert dokumentiert werden. Die Natur dieser komplexen Objekte, insbesondere ihre innere Dynamik während der Kollapsphase, konnte aufgrund der begrenzten Zeitauflösung der Meßapparatur noch nicht zweifelsfrei geklärt werden. Dennoch konnte gezeigt werden, daß diese Blasen-Cluster auf den experimentell im Rahmen dieser Arbeit zugänglichen 
Skalen stabile und durch eine attraktive Wechselwirkung gekoppelte, komplexe Mehrphasensysteme sind. Die Dynamik der Blasen-Cluster wird im wesentlichen durch Kollisionsereignisse zwischen den Clustern charakterisiert.

Neben dem Studium einzelner Trajektorien der Blasen erlaubt der hier vorgestellte Meßaufbau, die Dynamik eines gesamten Blasenfeldes über eine Zeitdauer von 7.3 Sekunden zu verfolgen. Auf der charakteristischen Zeitskala der Blasenbewegung, die durch die Periode $T$ der Anregung des Schallfeldes gegeben ist, entsprechen diesem Zeitraum etwa 1.6 $10^{5} T$. Damit ist die Aufnahmedauer um Größenordnungen größer als die typische Lebensdauer der Einzelblasen.

Die dynamischen Eigenschaften akustischer Kavitationsblasenfelder werden wesentlich durch die Verteilung der Ruheradien der Blasen charakterisiert. Diese Verteilung ist experimentell direkt nicht zugänglich, da in einer Messung nur die instantanen Radien der schwingenden Blasen bestimmt werden können. Mit dem in Kapitel 2 beschriebenen Verfahren konnten die Ruheradienverteilungen für verschiedene Parameterbereiche gemessen werden.

Mit den im Rahmen dieser Arbeit entwickelten experimentellen Methoden wurde eine Datenbasis der Positionen, Geschwindigkeiten, Radien und Kollisionsereignissen usw. in einem erheblichen und bisher nicht vorhandenen Umfang zur Verfügung gestellt. Damit ist eine wesentliche Voraussetzung geschaffen für die detaillierte Analyse der mikroskopischen und makroskopischen Dynamik dieses Systems. Damit ist aber auch erstmals die Validierung einer theoretischen Beschreibung akustischer Kavitationsblasenfelder mit Hilfe statistischer Methoden möglich.

Die erheblichen Datenmengen, die im Rahmen der experimentellen Untersuchungen anfielen, haben eine effiziente Implementierung der Bildverarbeitungsroutinen zwingend erforderlich gemacht. Daher wurden diese Routinen plattformunabhängig, skalier- und parallelisierbar implementiert und in einer Toolbox zusammengefaßt. Es wurde eine graphische Benutzeroberfläche entwickelt, damit die Routinen konfiguriert und einzelne Auswerteschritte vom Anwender exemplarisch durchgeführt und validiert werden können. Die so gewählten Bildbearbeitungs- und -analyseschritte können dann automatisiert auf einem Workstation-Cluster für die gesamte Bildsequenz durchgeführt werden.

Eine Erweiterung der in dieser Arbeit vorgestellten zweidimensionalen Messungen auf drei Raumdimensionen ist durch die Konzeption des experimentellen Aufbaus prinzipiell möglich und vorgesehen: Alle Algorithmen - die Kalibrierung des Kamerasystems, des PartikelTrackings und der Bildbearbeitung - sind so gewählt und implementiert, daß eine Verallgemeinerung auf eine stereoskopische Aufnahme und dreidimensionale Rekonstruktion erfolgen kann.

Motiviert durch die experimentellen Untersuchungen wurden im Kapitel 4 die Grundlagen der Modellierung akustischer Kavitationsblasenfelder diskutiert. Um die makroskopische Dynamik des Systems auf langsamen Zeitskalen zu beschreiben, wurde ein Kontinuumsmodell vorgestellt und diskutiert, das die wesentlichen Eigenschaften der Selbstfokussierung der Schallwelle und die Selbstkonzentration des Blasenfeldes beschreibt.

Allerdings liefert es keine Information über die Dynamik der Einzelblase und die Kopplung der Blasen untereinander. Daher wurde in dieser Arbeit auf der Basis der Lagrangeschen Formulierung der inkompressiblen Hydrodynamik ein Partikelmodell entwickelt. Dieses Modell erlaubt die Beschreibung der Kopplung von Translation und Oszillation einer Einzelblase und ihre Wechselwirkung mit anderen Blasen, solange die Grundannahmen des Modells erfüllt bleiben. Diese wurden diskutiert und darüber hinaus eine dynamische Stabilitätsgrenze für die Translationsbewegung der Blasen gegeben.

Im Rahmen dieses Modells werden die Wechselwirkungen der Blasen als MultipolWechselwirkungen beschrieben. Die Monopol-Monopol-, die Dipol-Dipol- und die Monopol- 
Dipol- Wechselwirkung wurden eingehend diskutiert und die Vorhersagen des Modells durch Vergleich mit der Literatur validiert. Das in dieser Arbeit formulierte Partikelmodell stellt damit eine grundlegende Erweiterung und Verbesserung der bisher vorgeschlagenen Modelle dar.

Das Partikelmodell erlaubt eine im Rahmen der Modellannahmen detaillierte Beschreibung der nichtlinearen mikroskopischen Wechselwirkung der Phasen. Es kann damit Grundlage für die Charakterisierung effektiver mittlerer Eigenschaften im Rahmen eines Kontinuummodells sein.

Da die Berücksichtigung des Einflusses der Kompressibilität der Flüssigkeit im Rahmen des vorgestellten Partikelmodells nicht möglich ist, wurde dieser Effekt in einem separaten Modell diskutiert. Die aus der Kompressibilität folgende zeitverzögerte Kopplung der Blasen zeigt für ihre Monopol-Monopol-Wechselwirkung einen qualitativ neuen Charakter. Die Komplexität dieser Wechselwirkung wird insbesondere durch die Brechung ihrer Symmetrie deutlich erhöht.

Abschließend wurde in Kapitel 4 die zentrale Fragestellung der Dimensionsanalyse der akustischen Kavitationsblasenfelder aufgegriffen und mit den Methoden der nichtlinearen Zeitreihenanalyse bearbeitet. Es wurde an einem einfachen Modell demonstriert, daß bei großen Systemen getriebener Oszillatoren durch Phasenkohärenz die Attraktorrekonstruktionen auf mittleren und größeren Skalen niedrigdimensional sind. Die tatsächliche hohe Dimension des Systems bleibt in klein-sklaligen Oszillationen verborgen, die in der Regel experimentell nicht oder nur schwer zugänglich sind. Dieses allgemeine Phänomen wurde durch die Simulation von N=300 durch ein Schallfeld getriebener Blasen untersucht und diskutiert. 


\section{A. Particle-Tracking-Velocimetry-Toolbox}

Die Particle-Tracking-Velocimetry-Toolbox (PTV-Toolbox) ist eine allgemeine Umgebung zur Entwicklung und Implementierung von Algorithmen zur Bildbearbeitung, Bild- und Bewegungsanalyse. Sie wurde im Rahmen dieser Arbeit primär für die Untersuchung strömungsphysikalischer Fragestellungen entwickelt, ist jedoch durch ihre allgemeinen Konzeption nicht auf diese Problemklasse beschränkt. In diesem Anhang soll ein kurzer Überblick über das Design und die Funktionalität der PTV-Toolbox gegeben werden.

Die folgenden in Kapitel 2 beschriebenen funktionalen Anforderungen an ein bildbearbeitendes System und Algorithmen wurden in der PTV-Toolbox implementiert:

- Bildbearbeitung. Elementare Routinen zur pixelorientierten oder geometrischen Bearbeitung.

- Bild- und Bewegungsanalyse. Neben dem modellbasierten Verfolgen von Objekten durch Tracking-Algorithmen besteht die Möglichkeit der grauwertorientierten Bewegungsanalyse mit Struktur-Tensor-Methoden.

- Datenvisualisierung. Für jeden Schritt der Bildbearbeitung, Bild- und Bewegungsanalye besteht die Möglichkeit der graphischen Darstellung.

- Photogrammetrie. Hierzu zählen Routinen zur Kalibrierung der Geometrie der bildgebenden Komponenten und der Rekonstruktion dreidimensionaler Objektpunkte.

- Datenbankanbindung. Die erheblichen Datenmengen, die im Rahmen der Bildaufnahme entstehen, und die Auswertungen können in einer Datenbank im HDF-Format gespeichert werden.

Diese funktionalen Anforderungen werden ergänzt durch die folgenden, allgemeinen Implementationsmerkmale der Toolbox:

- Plattformunabhängigkeit. Unterschiedliche Hardware-Architekturen und Betriebssysteme für die Datenaufnahme im Labor sowie die Datenverarbeitung, Visualisierung und Archivierung auf dem Workstation-Cluster machen eine plattformunabhängige Implementation erforderlich.

- Parallelisierbarkeit. Die erheblichen Datenmengen können auf dem Workstation-Cluster parallel verarbeitet werden. Dies ist durch Client-Server-Applikationen mit RemoteProcedure-Calls (RPCs) oder im Batch-Betrieb möglich.

- Graphische Benutzeroberfläche (GUI). Für die Erprobung und die Parametrisierung der Routinen sowie die Kontrolle während der Analyse ist die Interaktion mit dem Anwender notwendig. 


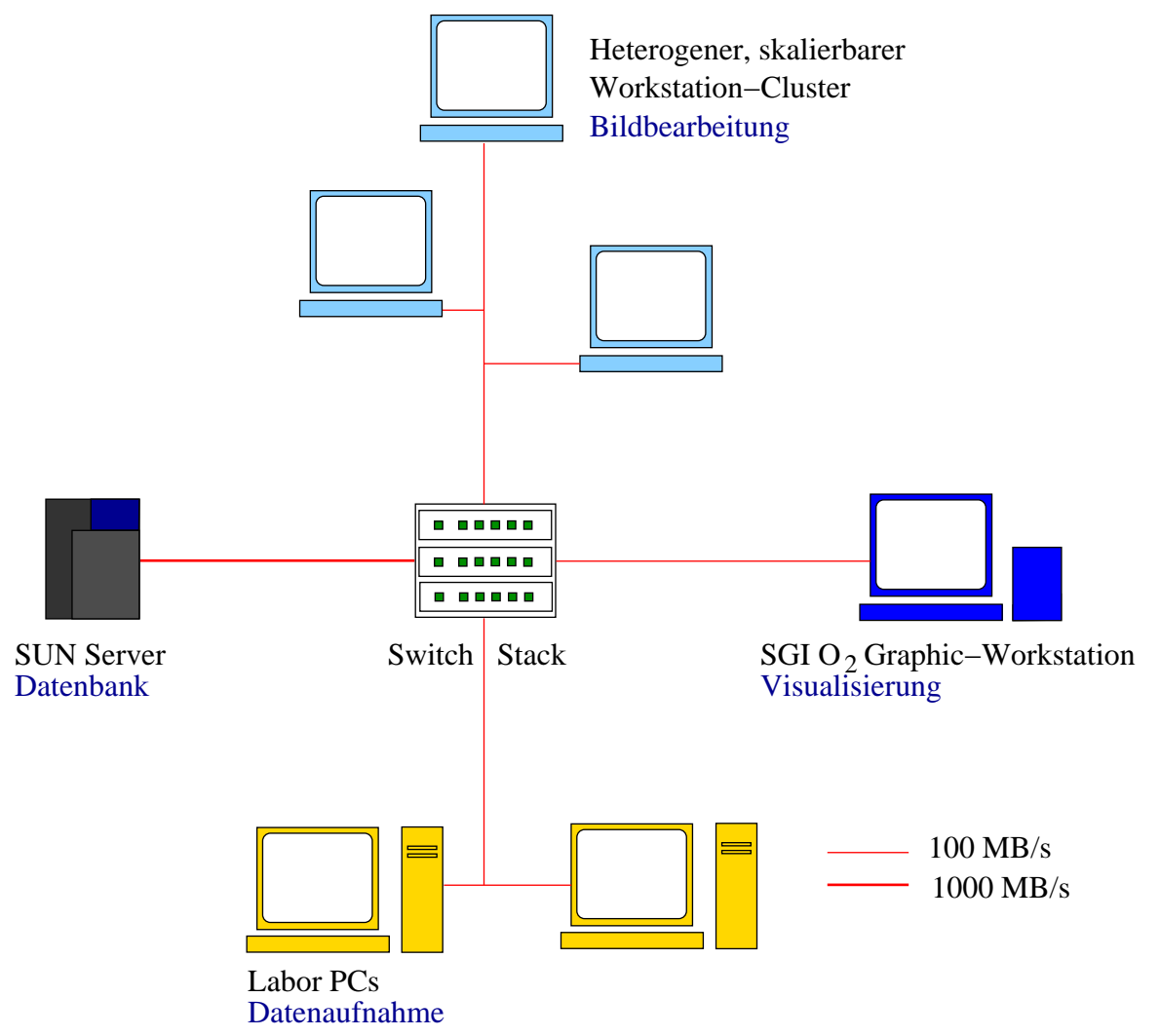

Abbildung A.1.: Schematische Darstellung der Datenverbeitung.

- Modularität und Erweiterbarkeit. Die Definition grundlegender Datenstrukturen und Schnittstellen und die modulare Implementation erlaubt die Erweiterung der Toolbox.

- Dokumentation. Um die Implementation der Algorithmen und ihre Verwendung für den Anwender und Entwickler nachvollziehbar zu halten, ist eine umfangreiche Dokumentation erstellt worden.

Die PTV-Toolbox wurde in der Programmiersprache PV-WAVE entwickelt, die für die Implementierung von Algorithmen der Bildbearbeitung und Visualisierung besonders geeignet ist. Geschwindigkeitskritische Programmteile wurden in $\mathrm{C}$ geschrieben und können als shared libraries dynamisch zum Programmcode hinzugebunden werden.

Abb. A.1 zeigt einen Ausschnitt der EDV-Infrastruktur, soweit sie für diese Arbeit relevant ist. Die Komponenten lassen sich nach ihrer Funktion gruppieren. Die Steuerung und Kontrolle des Experiments und die Datenaufnahme erfolgt mit den Labor-PCs. Die Meßdaten können auf einem zentralen Server in einer Datenbank gespeichert und verwaltet werden.

Eine Abschätzung des Bedarfs an Massenspeicher und Prozessorleistung zeigt den mit der Auswertung der Bilddaten verbundenen erheblichen Aufwand. Einer Aufnahme von 7.3 s Dauer entsprechen ca. $500 \mathrm{Mb}$ (unkomprimierter) Rohdaten, in einer Sequenz von ca. 16000 Einzelbildern. In dieser Sequenz werden etwa $10^{5}$ Objekte erkannt, deren dynamische und geometrische Eigenschaften bestimmt werden müsssen.

Für die z.T. mit erheblichem Rechenaufwand verbundene Daten- bzw. Bildbearbeitung und analyse steht ein skalierbarer, heterogener Workstation-Cluster zur Verfügung. Die Datenanalyse 


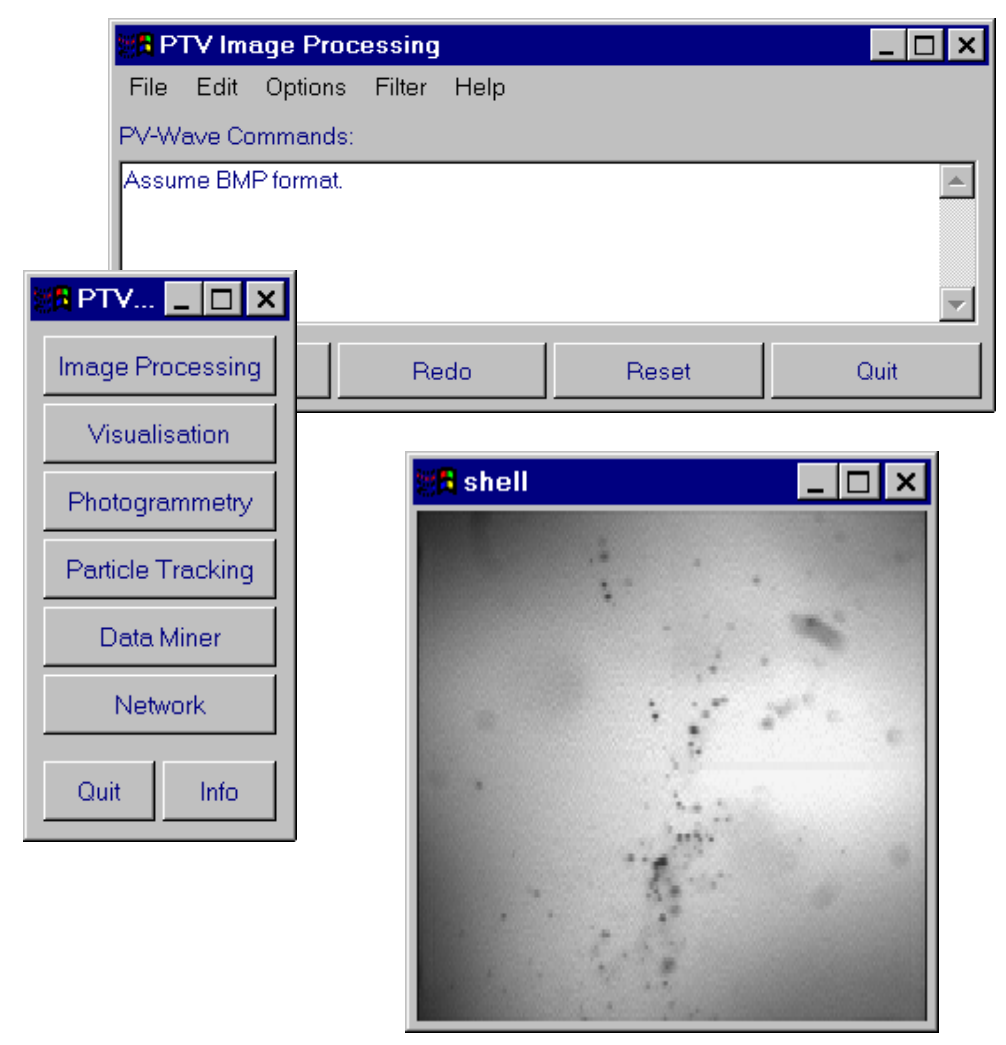

Abbildung A.2.: Bildbearbeitungsroutinen der PTV-Toolbox. Die einzelnen Schritte der Bildbearbeitung können interaktiv parametrisiert werden. Die Abfolge der Bearbeitungsschritte kann dann als Makro auf die gesamte Sequenz angewandt werden.

kann i.d.R. nicht ohne Interaktion durch den Anwender durchgeführt werden. Daher ist eine Visualisierung in allen Schritten der Bearbeitung notwendig. Insbesondere die Darstellung der Ergebnisse der dreidimensionalen Rekonstruktion erfolgt auf einer leistungsfähigen GraphikWorkstation. 


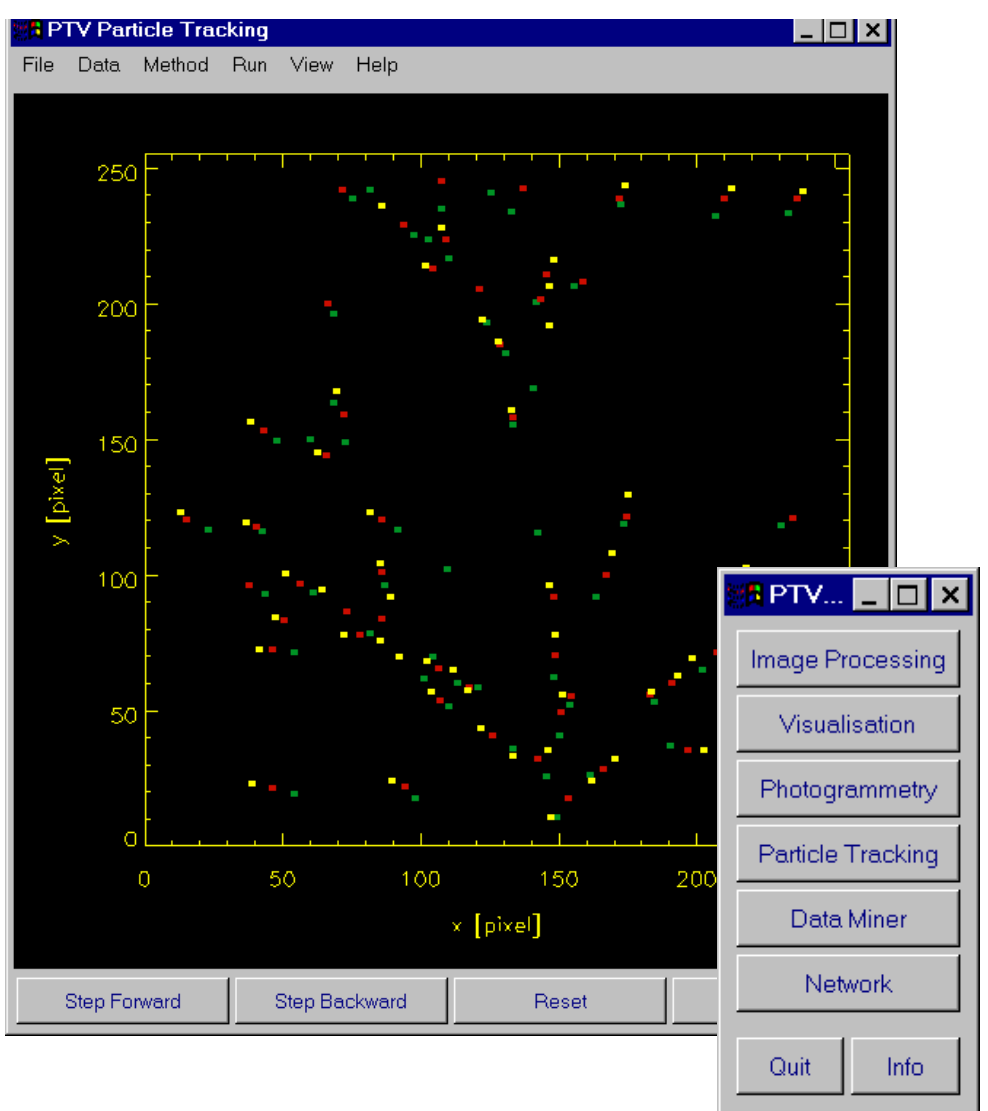

Abbildung A.3.: Particle-Tracking-Routinen der PTV-Toolbox. Die Trajektorien der Objekte können interaktiv festgelegt, dargestellt und gespeichert werden. 


\section{Literaturverzeichnis}

[1] H.D.I. Abarbanel: Analysis of Observed Chaotic Data. Springer Verlag, New York, 1996.

[2] I. Akhatov, U. Parlitz und W. Lauterborn: Pattern formation in acoustic cavitation. $J$. Acoust. Soc. Am. 96, 3627-3635, 1994.

[3] I. Akhatov, U. Parlitz und W. Lauterborn: Towards a theory of self-organization phenomena in bubble-liquid mixtures. Phys. Rev. E 54, 4990-5003, 1996.

[4] A.B. Basset: On the motion of two spheres in a liquid. Proc. London Math. Soc. 18, 369 $381,1887$.

[5] G.K. Batchelor: Transport properties of two-phase materials with random structure. Annu. Rev. Fluid Mech. 6, 227-255, 1974.

[6] M. Bentwich und T. Miloh: On the exact solution for the two sphere problem in axisymmetrical potential flow. Trans. ASME, J. Applied Mech. 45, 463-468, 1978.

[7] J.P. Best und J.R. Blake: An estimate of the Kelvin impulse of a transient cavity. J. Fluid Mech. 261, 75-93, 1994.

[8] M.D. Bethea, J.A. Lock, F. Merat und P. Crouser: Three-dimensional camera calibration technique for stereo imaging velocimetry experiments. Opt. Eng. 36, 3445-3454, 1997.

[9] A. Biesheuvel und L. van Wijngaarden: The motion of pairs of gas bubbles in a perfect liquid. J. Engng. Math. 16, 349-365, 1982.

[10] A. Billo: Holographische Partikelfeldanalyse am Beispiel Akustischer LichtenbergFiguren. Dissertation, TU Darmstadt, 1997.

[11] V.F. Bjerknes: Fields of Force. Columbia University Press, New York, 1906.

[12] J.R. Blake: The Kelvin impulse: Applications to cavitation bubble dynamics. J. Austral. Math. Soc. Ser. B 30, 127-146, 1988.

[13] C. Bongiovanni, J.P. Chevallier und J. Fabre: Sizing of bubbles by incoherent imaging: defocus bias. Exp. Fluids 23, 209-216, 1997.

[14] B. Brunner und G. Trygvason: Direct numerical simulation of three-dimensional bubbly flows. Phys. Fluids 11, 1967-1969, 1999.

[15] R.E. Calfisch, M.J. Miksis, G.C. Papanicolaou und Ting L.: Effective equations for wave propagation in bubbly liquids. J. Fluid Mech. 153, 259-273, 1985.

[16] R.B. Chapman und M.S. Plesset: Thermal effects in the free oscillation of gas bubbles. ASME J. Basic Eng. 93, 373-376, 1971.

[17] R.H. Cole: Underwater Explosions. Princeton University Press, 1948. 
[18] K.W. Commander und A. Prosperetti: Linear pressure waves in bubbly liquids: Comparison between theory and experiment. J. Acoust. Soc. Am. 85, 732-756, 1989.

[19] L.A. Crum: The polytropic exponent of gas contained within air bubbles pulsating in a liquid. J. Acoust. Soc. Am. 73, 116-120, 1983.

[20] C. Devin: Survey of thermal, radiation, and viscous damping of pulsationg air bubbles in water. J. Acoust. Soc. Am. 31, 1654-1667, 1959.

[21] R. Duraiswami, S. Prabhukumar und G.L. Chahine: Bubble counting using an inverse acoustic scattering method. J. Acoust. Soc. Am. 104(5), 2699-2717, 1998.

[22] H. Eckelmann: Einführung in die Strömungsmeßtechnik. Teubner Verlag, Stuttgart, 1997.

[23] J. Feng, H.H. Hu und D.D. Joseph: Direct simulation of initial value problem for motion of solid bodies in Newtonian fluid part 1. sedimentation. J. Fluid Mech. 261, 95-134, 1994.

[24] H. Goldstein: Klassische Mechanik. AULA-Verlag, Wiesbaden, 10. Aufl., 1989.

[25] J.F. Harper: On bubbles rising in line at large Reynolds numbers. J. Fluid Mech 41, 751758,1970 .

[26] J.F. Harper: Bubbles rising in a line: Why is the first approximation so bad? J. Fluid Mech. 351, 289-300, 1997.

[27] R.A. Hartunian und W.R. Sears: On the instability of small gas bubbles moving uniformly in various liquids. J. Fluid Mech. 3, 24-47, 1975.

[28] Y.A. Hassan und O.G. Philip: A new artificial neural network tracking technique for particle image velocimetry. Exp. Fluids 23, 145-154, 1997.

[29] F. Hering: Lagrangesche Untersuchung des Strömungsfeldes unterhalb der wellenbewegten Wasseroberfläche mittels Bildfolgenanalyse. Dissertation, Universität Heidelberg, 1996.

[30] W.M. Hicks: On the motion of two spheres in a liquid. Phil. Trans. R. Soc. London 171, 455-470, 1880.

[31] J. Holzfuss und W. Lauterborn: Liapunuov exponents from a time series of acoustic chaos. Phys. Rev. A 39, 2146-2152, 1989.

[32] H.H. Hu und D.D. Joseph: Direct simulation of fluid particles motion. Th. Comp. Fluid Dyn. 3, 285-306, 1992.

[33] B. Jähne: Spatio-Temporal Image Processing. Nr. 751 in Lecture Notes in Computer Science, Springer-Verlag, Heidelberg, 1993.

[34] B. Jähne: Digitale Bildverarbeitung. Springer-Verlag, Heidelberg, 4. Aufl., 1997.

[35] G. Jetschke: Mathematik der Selbstorganisation. Vieweg, Braunschweig, 1989.

[36] H. Kantz und T. Schreiber: Nonlinear Time Series Analysis. Cambridge University Press, 1997.

[37] J.B. Keller und M. Miksis: Bubble oscillations of large amplitude. J. Acoust. Soc. Am. 68, 628-633, 1980. 
[38] H.S. Kim und A. Prosperetti: Numerical simulation of the motion of rigid spheres in potential flow. SIAM J. Appl. Math. 52, 1533-1562, 1992.

[39] H. Knutsson: Filtering and reconstruction in image processing. Dissertation, Univ. Linköping, 1989.

[40] J.B.W. Kok: Dynamics of a pair of bubbles moving through liquid, I Theory. Eur. J. Mech., B/Fluids 4, 541-560, 1993.

[41] J.B.W. Kok: Dynamics of a pair of bubbles moving through liquid, II Experiment. Eur. J. Mech., B/Fluids 4, 515-550, 1993.

[42] V. Kumaran und D.L. Koch: The effect of hydrodynamic interactions on the average properties of a bisphere suspension of high reynolds number, low weber number bubbles. Phys. Fluids A 5, 1123-1134, 1993.

[43] H. Lamb: Hydrodynamics. Cambridge University Press, Cambridge, 6. Aufl., 1932.

[44] L.D. Landau und E.M. Lifschitz: Lehrbuch der Theoretischen Physik, Bd. I. AkademieVerlag, Berlin, 1990.

[45] L.D. Landau und E.M. Lifschitz: Lehrbuch der Theoretischen Physik, Bd. VI. AkademieVerlag, Berlin, 5. Aufl., 1991.

[46] W. Lauterborn, Hg.: Cavitation and Inhomogeneities in Underwater Acoustics. Springer Verlag, Heidelberg, 1980.

[47] W. Lauterborn und H. Bolle: Experimental investigations of cavitation bubble collapse in the neighbourhood of a solid boundary. J. Fluid Mech. 72, 391-399, 1975.

[48] W. Lauterborn und J. Holzfuss: Evidence for a low-dimensional strange attractor in acoustic turbulence. Phys. Lett. A 115, 369-372, 1986.

[49] V.G. Levich: Physicochemical Hydrodynamics. Prentice-Hall, New York, 1962.

[50] S. Luther, R. Mettin und W. Lauterborn: Modelling acoustic cavitation by a Lagrangian approach. In: Proc. ISNA 15, Göttingen, (im Druck), 1999.

[51] S. Luther, U. Parlitz, I. Akhatov und W. Lauterborn: Strukturbildung in akustischen kavitationsblasenfeldern. In: Fortschritte der Akustik, S. 339-340, P.Wille; Oldenburg: DEGA e.V., 1997.

[52] S. Luther, U. Parlitz, M. Sushchik, I. Akhatov und W. Lauterborn: Is cavitation noise governed by a low dimensional chaotic attractor? In: Proc. ISNA 15, Göttingen, (im Druck), 1999.

[53] E.P. Lywers, O.R. Mitchell, M.L. Akey und A.P. Reeves: Subpixel measurements using a moment-based edge operator. IEEE Trans. PAMI 11, 1293-1309, 1989.

[54] J. Magnaudet und D. Legendre: The viscous drag force on a spherical bubble with timedependent radius. Phys. Fluids 10, 550-554, 1998.

[55] R. Mettin, I. Akhatov, U. Parlitz, C.D. Ohl und W. Lauterborn: Bjerknes forces between small caviation bubbles in a strong acoustic field. Phys. Rev. E 56(3), 2924-2931, 1997.

[56] R. Mettin, S. Luther, S. Kamphausen und W. Lauterborn: Dynamics of delay-coupled spherical bubbles. In: Proc. ISNA 15, Göttingen, (im Druck), 1999. 
[57] R. Mettin, S. Luther und W. Lauterborn: Bubble size distributions and structures in acoustic cavitation. In: 2nd Conference on Ultrasound in Processing, S. 125-129, Toulouse, France, 1999.

[58] T. Miloh: Hydrodynamics of deformable contigous spherical shapes in an incompressible inviscid liquid. J. Eng. Math. 11, 349-372, 1977.

[59] D.W. Moore: The boundary layer on a sperical gas bubble. J. Fluid Mech. 16, 161-176, 1963.

[60] D.W. Moore: The velocity of rise of distorted gas bubbles in a liquid of small viscosity. $J$. Fluid Mech. 23, 749-766, 1965.

[61] R.I. Nigmatulin: Dynamics of Multiphase Media. Hemisphere Publ. Corp., New York, 1991.

[62] H.N. Oguz und A. Prosperetti: A generalization of the impulse and virial theorems with application to bubble oscillations. J. Fluid Mech. 218, 143-162, 1990.

[63] U. Parlitz, V. Englisch, C. Scheffczyk und W. Lauterborn: Bifurcation structure of bubble oscillators. J. Acoust. Soc. Am. 88, 1061-1077, 1990.

[64] U. Parlitz, R. Mettin, S. Luther, M. Voss und W. Lauterborn: Spatio-temporal dynamics of acoustic cavitation bubble clouds. Phil. Trans. R. Soc. Lond. A 357, 313-334, 1999.

[65] A.K. Prasad und R.J. Adrian: Stereoscopic particle image velocimetry applied to liquid flows. Exp. Fluids 15, 49-60, 1993.

[66] A. Prosperetti: Thermal effects and damping mechanisms in the forced radial oscillations of gas bubbles in liquids. J. Acoust. Soc. Am. 61, 17-27, 1977.

[67] T.H. Reiss: Recognizing planar objects using invariant image features. Nr. 676 in Lecture Notes in Computer Science, Springer-Verlag, Heidelberg, 1993.

[68] D. Ruelle: Deterministic chaos: the science and the fiction. Proc. R. Soc. Lond. A 427, 241-248, 1990.

[69] G. Russo und S. Smereka: Kinetic theory for bubbly flow I: Collisionsless case. SIAM J. Appl. Math. 56(2), 327-357, 1996.

[70] G. Russo und S. Smereka: Kinetic theory for bubbly flow II: Fluid dynamic limit. SIAM J. Appl. Math. 56(2), 358-371, 1996.

[71] G. Ryskin und L.G. Leal: Numerical solution of free-boundary problems in fluid mechanics. Part 1. The finite difference techniques. J. Fluid Mech. 148, 1-17, 1984.

[72] P.G. Saffman: On the rise of small air bubbles in water. J. Fluid Mech. 1, 249-275, 1956.

[73] A.S. Sangani und A.K. Didwania: Dynamic simulations of flows of bubbly liquids at large Reynolds numbers. J. Fluid Mech. 250, 307-337, 1993.

[74] E. Schmitz: Raum-zeitliche Strukturerfassung von holokinematisch aufgezeichneten Kavitationsblasenfeldern mit Hilfe der digitalen Bildverarbeitung. Dissertation, TU Darmstadt, 1997.

[75] P. Smereka: On the dynamics of bubbles in a periodic box. J. Fluid Mech. 254, 79-112, 1993. 
[76] A. Sommerfeld: Vorlesungen über theoretische Physik, Bd. I. Geest \& Portig, Leipzig, 3. Aufl., 1947.

[77] Y. Tsuji, Y. Morikawa und K. Terashima: Fluid dynamic interaction between two spheres. Int. J. Multiphase Flow 8, 71-82, 1982.

[78] O.V. Voinov: On the motion of two spheres in a perfect liquid. PMM 33, 659-667, 1969.

[79] M. Voß: Selbstfokussierung bei der akustischen Kavitation. Diplomarbeit, Universität Göttingen, 1998.

[80] L. van Wijngaarden: On equations of motion for mixtures of liquid and gas bubbles. $J$. Fluid Mech. 33, 465-474, 1968.

[81] L. van Wijngaarden: The mean rise velocity of pairwise interaction bubbles in liquid. $J$. Fluid Mech. 251, 55-78, 1993.

[82] C.E. Willert und M. Gharib: Digital particle image velocimetry. Exp. Fluids 10, 181-193, 1991.

[83] M. Wilson und J.R. Blake: A potential multipole theory for the hydrodynamics of bubble clouds. J. Fluid Mech, (eingereicht), 1999.

[84] R.F. Young: Cavitation. McGraw-Hill Book Comp., London, 1989.

[85] T. Yuan und A. Prosperetti: On the in-line motion of two spherical bubbles in a viscous fluid. J. Fluid Mech. 278, 325-349, 1994.

[86] Y. Yurkovetsky und J.F. Brady: Statistical mechanics of bubbly liquids. Phys. Fluids 8, 881-895, 1996.

[87] E.A. Zabolotskaya: Interaction of gas bubbles in a sound field. Sov. Phys. Acoust. 30(5), 365-368, 1984. 


\section{Danksagung}

Ich danke Prof. Dr. Werner Lauterborn für die Ermöglichung dieser Arbeit. Seine engagierte Förderung durch die außergewöhnlich gute Ausstattung und sein stets offenes Ohr waren eine sehr gute Basis und Unterstützung für die Anfertigung dieser Arbeit.

Mein ganz besonderer Dank gilt PD Dr. Ulrich Parlitz und Dr. Robert Mettin für die intensiven Diskussionen und Anregungen.

Meinen Freunden und Kollegen Martin Wiesenfeldt, Lutz Junge, Jörg Wichard, Claus-Dieter Ohl und Christian Merkwirth danke ich für die gemeinsame Zeit.

Dagmar Krefting und Martin Voß sowie Reinhard Geisler und Philipp Koch danke ich für die sehr gute und freundschaftliche Zusammenarbeit im Labor.

Allen Mitgliedern der Arbeitsgruppe "Nichtlineare Dynamik" danke ich für die vielfältigen Diskussionen. Allen Mitgliedern des Institutes danke ich für die gemeinsam geschaffene kreative und angenehme Atmoshpäre, in der ich mich wohl gefühlt habe.

Insbesondere möchte ich allen Mitarbeitern der Elektronischen und Mechanischen Werkstätten für ihr Engagement danken, mit dem sie ihr technisches und handwerkliches Können und Geschick in diese Arbeit eingebracht und damit wesentlich zu ihrem Erfolg beigetragen haben.

Ich bin Prof. Dr. Iskander Akhatov, Prof. Dr. John Blake, Dr. Mikhail Sushckik und Dr. John Allen für viele anregende Diskussionen zu Dank verpflichtet.

Meinen meinen Eltern, Großeltern und Geschwistern gilt mein Dank für ihre Unterstützung und ihr Interesse an meiner Arbeit.

Von ganzem Herzen danke ich meiner Freundin Gisa Schäfer für ihre Geduld und Unterstützung und für alles, was Worte nicht fassen können.

Die vorliegende Arbeit wurde vom Graduiertenkolleg "Strömungsinstabilitäten und Turbulenz" an der Georg-August-Universität zu Göttingen unterstützt. 


\section{Lebenslauf}

Stefan Luther

25.03.1969 geboren in Springe

1975-1979 Besuch der Grundschule Springe

1979-1981 Besuch der Orientierungsstufe in Springe

1981-1988 Besuch des Otto-Hahn-Gymnasiums in Springe, Abschluß mit Abitur

1989-1990 Zivildienst im Annastift in Hannover

1990-1994 Studium der Physik an der Universität Hannover

1994-1997 Studium der Physik an der Universität Göttingen

Februar 1997 Abschluß des Hochschulstudiums mit dem Diplom

1997-2000 Stipendiat des Graduiertenkollegs "Strömungsinstabilitäten und Turbulenz" an der Georg-August-Universiät zu Göttingen 